Article

\title{
Solving Fuzzy Linear Programming Problems with Fuzzy Decision Variables
}

\author{
Hsien-Chung Wu \\ Department of Mathematics, National Kaohsiung Normal University, Kaohsiung 802, Taiwan; \\ hcwu@nknucc.nknu.edu.tw
}

Received: 26 May 2019; Accepted: 21 June 2019; Published: 26 June 2019

\begin{abstract}
The numerical method for solving the fuzzy linear programming problems with fuzzy decision variables is proposed in this paper. The difficulty for solving this kind of problem is that the decision variables are assumed to be nonnegative fuzzy numbers instead of nonnegative real numbers. In other words, the decision variables are assumed to be membership functions. One of the purposes of this paper is to derive the analytic formula of error estimation regarding the approximate optimal solution. On the other hand, the existence of optimal solutions is also studied in this paper. Finally we present two numerical examples to demonstrate the usefulness of the numerical method.
\end{abstract}

Keywords: discretized linear programming problems; fuzzy numbers; nondominated solutions; strong duality theorem; weak duality theorem

\section{Introduction}

Solving the fuzzy linear programming problem has received considerable attention for a long time. Bellman and Zadeh [1] first studied the fuzzy optimization problems by using the aggregation operators to combine the fuzzy goals and fuzzy decision space. Tanaka et al. [2], Zimmermann [3,4] and Herrera et al. [5] considered the aspiration level approach to study the fuzzy linear programming problems. Verdegay [6] studied the fuzzy primal and dual problems. Buckley [7], Julien [8] and Luhandjula et al. [9] solved the mathematical programming problems based on the possibility. Inuiguchi [10] studied the so-called modality constrained programming problems based on the possibility and necessity measures.

Considering the fuzzy coefficients in optimization problems is also an important issue, where the decision variables are still assumed to be nonnegative real numbers. Wu [11-14] used the so-called Hukuhara derivative to study the duality theorems and optimality conditions for fuzzy optimization problems. Li et al. [15] used the generalized convexity to study the optimality conditions. The Newton method was proposed by Chalco-Cano et al. [16] and Pirzada and Pathak [17] to solve the fuzzy optimization problems. In this paper, we are going to solve the fuzzy linear programming problems in which the decision variables are assumed to be nonnegative fuzzy numbers. This consideration will complicate the problem. The existing articles for studying the fuzzy linear programming problems with fuzzy decision variables always assume the fuzzy decision variables to be the triangular fuzzy numbers or trapezoidal fuzzy numbers by means of their simple forms. In this paper, the fuzzy decision variables are assumed to be the general bell-shaped fuzzy numbers. This is the first attempt to solve this kind of difficult problem.

Buckley and Feuring [7] used the evolutionary algorithm to solve the so-called fully fuzzified linear programming problems in which the coefficients and decision variables were assumed to be triangular fuzzy numbers. Ezzati et al. [18] studied the fully fuzzy linear programming problems in which the coefficients and decision variables were also assumed to be triangular fuzzy numbers by converting the original fuzzy problem into a multiobjective linear programming problems. 
Ahmad et al. [19], Jayalakshmi and Pandian [20], Khan et al. [21], Kumar et al. [22], Lotfi et al. [23], Najafi et al. [24] and Nasseri et al. [25] also studied the fully fuzzy linear programming problems based on triangular fuzzy numbers. Kaur and Kumar [26] studied the fully fuzzy linear programming problems based on trapezoidal fuzzy numbers. Baykasoglu and Gocken [27] used the particle swarm optimization method to solve the fuzzy mathematical programs with fuzzy decision variables in which only triangular fuzzy numbers were taken into account. The main reason for considering the triangular fuzzy numbers and trapezoidal fuzzy numbers is that they can be parametrically represented and formulated. In other words, the above methods will be invalid when the fuzzy quantities are not taken to be triangular fuzzy numbers or trapezoidal fuzzy numbers. This drawback will be overcome in this paper.

The fuzzy transportation problems are frequently formulated as fuzzy linear programming problems with fuzzy decision variables. However, the fuzzy quantities are still assumed to be triangular fuzzy numbers or trapezoidal fuzzy numbers for avoiding the complication. Chakraborty et al. [28] and Jaikumar [29] solved the fully fuzzy transportation problems using triangular fuzzy numbers. Baykasoglu and Subulan [30] studied the fuzzy transportation problems with fuzzy decision variables in which the fuzzy quantities are also taken to be triangular fuzzy numbers. They used the so-called constrained fuzzy arithmetic (ref. Klir and Pan [31]) to transform the fuzzy transportation problems with fuzzy decision variables into $\alpha$-level problems for $\alpha \in[0,1]$, where the $\alpha$-level problems are the crisp mathematical programming problems. Ebrahimnejad [32] and Kaur and Kumar [33] solved the fuzzy transportation problems using the so-called generalized trapezoidal fuzzy numbers. Kaur and Kumar [26] also studied the unbalanced fully fuzzy minimal cost flow problems in which the fuzzy quantities are taken to LR fuzzy numbers. The above existing methods did not consider the error between the optimal solution and numerical optimal solution. One of the contribution of this paper is to obtain the error bound between the optimal solution and numerical optimal solution.

The fuzzy linear programming problem with fuzzy decision variables is a difficult problem because of solving this kind of problem is that the decision variables are taken to be membership functions. In this paper, we shall use the basic properties of fuzzy numbers to transform the original fuzzy linear programming problem into a scalar optimization problem in which the decision variables are monotonic functions defined on the unit interval $[0,1]$. In order to solve this transformed scalar optimization problem, we shall consider its discretization by equally dividing the unit interval $[0,1]$ into subintervals, which can be formulated as a linear programming problem. One of the purposes of this paper is to derive the analytic formula of error estimation regarding the approximate optimal solution. Therefore, we need to study the dual problem of the discretized linear programming problem. The existence of optimal solutions is also studied in this paper. Some slight sufficient conditions are needed to guarantee the existence of optimal solutions.

This paper is organized as follows. In Section 2, the fuzzy linear programming problems with fuzzy decision variables are formulated. In order to solve this original problem, it is transformed into a scalar optimization problem. The solution concepts and some related properties will be established. In Section 3, we shall introduce a discretized problem of the transformed scalar optimization problem by equally dividing the unit interval $[0,1]$ into subintervals with equal lengths. The dual problem of this discretized problem will also be formulated in order to design the computational procedure. In Section 4, we shall derive an analytic formula of the error bound. The concept of asymptotic no duality gap and the related results will be established. In Section 5, we shall study the existence of optimal solutions by providing some slight sufficient conditions. In Section 6, we shall derive a tighter bound of error estimation for nonnegative case. In Sections 7 and 8, the computational procedures are proposed, and two numerical example are provided to demonstrate the usefulness of this practical algorithm. 


\section{Formulation}

The fuzzy subset $\widetilde{A}$ of $\mathbb{R}$ is defined by a membership function $\xi_{\widetilde{A}}: \mathbb{R} \rightarrow[0,1]$. The $\alpha$-level set of $\widetilde{A}$, denoted by $\widetilde{A}_{\alpha}$, is defined by

$$
\widetilde{A}_{\alpha}=\left\{x \in \mathbb{R}: \widetilde{\zeta}_{\widetilde{A}}(x) \geq \alpha\right\},
$$

for all $\alpha \in(0,1]$. The 0 -level set $\widetilde{A}_{0}$ is defined as the closure of the set $\left\{x \in \mathbb{R}: \xi_{\widetilde{A}}(x)>0\right\}$. It is clear to see that $\widetilde{A}_{\alpha} \subseteq \widetilde{A}_{\beta}$ for $\alpha>\beta$.

Let $\widetilde{A}$ and $\widetilde{B}$ be two fuzzy subsets of $\mathbb{R}$. According to the extension principle, the addition and multiplication between $\widetilde{A}$ and $\widetilde{B}$ are defined by

$$
\xi_{\widetilde{A} \oplus \widetilde{B}}(z)=\sup _{\{(x, y): z=x+y\}} \min \left\{\xi_{\widetilde{A}}(x), \xi_{\widetilde{B}}(y)\right\},
$$

and

$$
\xi_{\widetilde{A} \otimes \widetilde{B}}(z)=\sup _{\{(x, y): z=x * y\}} \min \left\{\tilde{\zeta}_{\widetilde{A}}(x), \xi_{\widetilde{B}}(y)\right\} .
$$

Let $\widetilde{a}$ be a fuzzy subset of $\mathbb{R}$. We say that $\widetilde{a}$ is a fuzzy number if and only if the following conditions are satisfied:

- $\quad \tilde{a}$ is normal, i.e., $\xi_{\widetilde{a}}(x)=1$ for some $x \in \mathbb{R}$;

- $\quad \tilde{a}$ is convex, i.e., the membership function $\xi_{\widetilde{a}}(x)$ is quasi-concave;

- the membership function $\xi_{\widetilde{a}}$ is upper semicontinuous;

- the 0 -level set $\widetilde{a}_{0}$ is a closed and bounded subset of $\mathbb{R}$.

It is well-known that each $\alpha$-level sets $\widetilde{a}_{\alpha}$ of a fuzzy number $\widetilde{a}$ is a bounded closed interval in $\mathbb{R}$, which is also denoted by

$$
\widetilde{a}_{\alpha}=\left[\widetilde{a}_{\alpha}^{L}, \widetilde{a}_{\alpha}^{U}\right]
$$

Remark 1. Let a be a fuzzy number. Then we have the following properties:

- $\widetilde{a}_{\alpha}^{L} \leq \widetilde{a}_{\alpha}^{U}$ for all $\alpha \in[0,1]$;

- $\widetilde{a}_{\alpha}^{L}$ is increasing with respect to $\alpha$ on $[0,1]$;

- $\quad \tilde{a}_{\alpha}^{U}$ is decreasing with respect to $\alpha$ on $[0,1]$.

We say that $\widetilde{a}$ is a nonnegative fuzzy number if and only if $\widetilde{a}_{\alpha}^{L} \geq 0$ for all $\alpha \in[0,1]$; that is, if $r<0$ then $\xi_{\widetilde{a}}(r)=0$. Similarly, we say that $\widetilde{a}$ is a nonpositive fuzzy number if and only if $\widetilde{a}_{\alpha}^{U} \leq 0$ for all $\alpha \in[0,1]$; that is, if $r>0$ then $\widetilde{\zeta}_{\widetilde{a}}(r)=0$.

Let $\widetilde{a}$ and $\widetilde{b}$ be two fuzzy numbers. Then

$$
(\widetilde{a} \oplus \widetilde{b})_{\alpha}=\widetilde{a}_{\alpha}+\widetilde{b}_{\alpha}=\left[\widetilde{a}_{\alpha}^{L}, \widetilde{a}_{\alpha}^{U}\right]+\left[\widetilde{b}_{\alpha}^{L}, \widetilde{b}_{\alpha}^{U}\right]=\left[\widetilde{a}_{\alpha}^{L}+\widetilde{b}_{\alpha}^{L}, \widetilde{a}_{\alpha}^{U}+\widetilde{b}_{\alpha}^{U}\right] \text { for } \alpha \in[0,1],
$$

and

$$
\begin{aligned}
(\widetilde{a} \otimes \widetilde{b})_{\alpha} & =\widetilde{a}_{\alpha} * \widetilde{b}_{\alpha}=\left[\widetilde{a}_{\alpha}^{L}, \widetilde{a}_{\alpha}^{U}\right] *\left[\widetilde{b}_{\alpha}^{L}, \widetilde{b}_{\alpha}^{U}\right] \\
& =\left[\min \left\{\widetilde{a}_{\alpha}^{L} \widetilde{b}_{\alpha}^{L}, \widetilde{a}_{\alpha}^{L} \widetilde{b}_{\alpha}^{U}, \widetilde{a}_{\alpha}^{U} \widetilde{b}_{\alpha}^{L}, \widetilde{a}_{\alpha}^{U} \widetilde{b}_{\alpha}^{U}\right\}, \max \left\{\widetilde{a}_{\alpha}^{L} \widetilde{b}_{\alpha}^{L}, \widetilde{a}_{\alpha}^{L} \widetilde{b}_{\alpha}^{U}, \widetilde{a}_{\alpha}^{U} \widetilde{b}_{\alpha}^{L}, \widetilde{a}_{\alpha}^{U} \widetilde{b}_{\alpha}^{U}\right\}\right] \text { for } \alpha \in[0,1] .
\end{aligned}
$$

In particular, if $\widetilde{b}$ is a nonnegative fuzzy number, then

$$
(\widetilde{a} \otimes \widetilde{b})_{\alpha}=\left[\min \left\{\widetilde{a}_{\alpha}^{L} \widetilde{b}_{\alpha}^{L}, \widetilde{a}_{\alpha}^{L} \widetilde{b}_{\alpha}^{U}\right\}, \max \left\{\widetilde{a}_{\alpha}^{U} \widetilde{b}_{\alpha}^{L}, \widetilde{a}_{\alpha}^{U} \widetilde{b}_{\alpha}^{U}\right\}\right] \text { for } \alpha \in[0,1] .
$$

Therefore we can also consider the following two cases. 
- $\quad$ Suppose that $\widetilde{a}$ and $\widetilde{b}$ are nonnegative fuzzy numbers. Then

$$
(\widetilde{a} \otimes \widetilde{b})_{\alpha}=\left[\widetilde{a}_{\alpha}^{L} \widetilde{b}_{\alpha}^{L}, \widetilde{a}_{\alpha}^{U} \widetilde{b}_{\alpha}^{U}\right] \text { for } \alpha \in[0,1] .
$$

- Suppose that $\widetilde{a}$ is a nonpositive fuzzy number and $\widetilde{b}$ is a nonnegative fuzzy numbers. Then

$$
(\widetilde{a} \otimes \widetilde{b})_{\alpha}=\left[\widetilde{a}_{\alpha}^{L} \widetilde{b}_{\alpha}^{U}, \widetilde{a}_{\alpha}^{U} \widetilde{b}_{\alpha}^{L}\right] \text { for } \alpha \in[0,1]
$$

Let $\widetilde{a}_{j}, \widetilde{b}_{i j}$ and $\widetilde{c}_{i}$ be fuzzy numbers for $i=1, \cdots, p$ and $j=1, \cdots, q$. For convenience, we write the $\alpha$-level sets of them as

$$
\left(\widetilde{a}_{j}\right)_{\alpha}^{L}=\widetilde{a}_{j \alpha}^{L}, \quad\left(\widetilde{a}_{j}\right)_{\alpha}^{U}=\widetilde{a}_{j \alpha}^{U}, \quad\left(\widetilde{b}_{i j}\right)_{\alpha}^{L}=\widetilde{b}_{i j \alpha}^{L} \quad\left(\widetilde{b}_{i j}\right)_{\alpha}^{U}=\widetilde{b}_{i j \alpha}^{U}, \quad\left(\widetilde{c}_{i}\right)_{\alpha}^{L}=\widetilde{c}_{i \alpha}^{L} \text { and }\left(\widetilde{c}_{i}\right)_{\alpha}^{U}=\widetilde{c}_{i \alpha}^{U} .
$$

Now we consider the following fuzzy linear programming problem with fuzzy decision variables $\widetilde{x}_{1}, \cdots, \widetilde{x}_{q}:$

$$
\begin{aligned}
(\mathrm{FLP}) \quad \max \quad & \left(\widetilde{a}_{1} \otimes \widetilde{x}_{1}\right) \oplus\left(\widetilde{a}_{2} \otimes \widetilde{x}_{2}\right) \oplus \cdots \oplus\left(\widetilde{a}_{q} \otimes \widetilde{x}_{q}\right) \\
\text { subject to } & \left(\widetilde{b}_{i 1} \otimes \widetilde{x}_{1}\right) \oplus\left(\widetilde{b}_{i 2} \otimes \widetilde{x}_{2}\right) \oplus \cdots \oplus\left(\widetilde{b}_{i q} \otimes \widetilde{x}_{q}\right) \preccurlyeq \widetilde{c}_{i} \text { for } i=1, \cdots, p ; \\
& \text { each } \widetilde{x}_{j} \text { is a nonnegative fuzzy number for } j=1, \cdots, q,
\end{aligned}
$$

where the partial ordering $\preccurlyeq$ appeared in the constraints will be interpreted below by considering their $\alpha$-level sets. Let

$$
\widetilde{f}(\widetilde{\mathbf{a}}, \widetilde{\mathbf{x}})=\left(\widetilde{a}_{1} \otimes \widetilde{x}_{1}\right) \oplus\left(\widetilde{a}_{2} \otimes \widetilde{x}_{2}\right) \oplus \cdots \oplus\left(\widetilde{a}_{q} \otimes \widetilde{x}_{q}\right)
$$

and

$$
\widetilde{g}_{i}\left(\widetilde{\mathbf{b}}_{i}, \widetilde{\mathbf{x}}\right)=\left(\widetilde{b}_{i 1} \otimes \widetilde{x}_{1}\right) \oplus\left(\widetilde{b}_{i 2} \otimes \widetilde{x}_{2}\right) \oplus \cdots \oplus\left(\widetilde{b}_{i q} \otimes \widetilde{x}_{q}\right) \text { for } i=1, \cdots, p .
$$

Then, by considering the lower and upper bounds of $\alpha$-level sets of constraints, the fuzzy linear programming (FLP) problem is interpreted as follows:

$$
\begin{aligned}
(\text { FLP }) \quad \max \quad & \widetilde{f}(\widetilde{\mathbf{a}}, \widetilde{\mathbf{x}}) \\
\text { subject to } & \left(\widetilde{g}_{i}\left(\widetilde{\mathbf{b}}_{i}, \widetilde{\mathbf{x}}\right)\right)_{\alpha}^{L} \leq \widetilde{c}_{i \alpha}^{L} \text { for } i=1, \cdots, p \text { and } \alpha \in[0,1] ; \\
& \left(\widetilde{g}_{i}\left(\widetilde{\mathbf{b}}_{i}, \widetilde{\mathbf{x}}\right)\right)_{\alpha}^{U} \leq \widetilde{c}_{i \alpha}^{U} \text { for } i=1, \cdots, p \text { and } \alpha \in[0,1] ; \\
& \text { each } \widetilde{x}_{j} \text { is a nonnegative fuzzy number for } j=1, \cdots, q .
\end{aligned}
$$

We assume that each $\widetilde{b}_{i j}$ is a nonnegative or nonpositive fuzzy number. For each $i=1, \cdots, p$, we define the sets of indices as follows:

$$
P_{i}=\left\{j: \widetilde{b}_{i j} \text { is a nonnegative fuzzy number }\right\},
$$

and

$$
N_{i}=\left\{j: \widetilde{b}_{i j} \text { is a nonpositive fuzzy number }\right\} .
$$

It is clear to see that $P_{i} \cup N_{i}=\{1,2, \cdots, q\}$ for $i=1, \cdots, p$. 
Using (1)-(3), the problem FLP can be written as follows:

$$
\begin{array}{cl}
\max & \widetilde{f}(\widetilde{\mathbf{a}}, \widetilde{\mathbf{x}}) \\
\text { subject to } & \sum_{\left\{j: j \in P_{i}\right\}} \widetilde{b}_{i j \alpha}^{L} \cdot \widetilde{x}_{j \alpha}^{L}+\sum_{\left\{j: j \in N_{i}\right\}} \widetilde{b}_{i j \alpha}^{L} \cdot \widetilde{x}_{j \alpha}^{U} \leq \widetilde{c}_{i \alpha}^{L} \text { for } i=1, \cdots, p \text { and } \alpha \in[0,1] ; \\
& \sum_{\left\{j: j \in N_{i}\right\}} \widetilde{b}_{i j \alpha}^{U} \cdot \widetilde{x}_{j \alpha}^{L}+\sum_{\left\{j: j \in P_{i}\right\}} \widetilde{b}_{i j \alpha}^{U} \cdot \widetilde{x}_{j \alpha}^{U} \leq \widetilde{c}_{i \alpha}^{U} \text { for } i=1, \cdots, p \text { and } \alpha \in[0,1] ; \\
& \text { each } \widetilde{x}_{j} \text { is a nonnegative fuzzy number for } j=1, \cdots, q .
\end{array}
$$

Since the decision variables $\widetilde{x}_{j}$ for $j=1, \cdots, q$ are assumed to be nonnegative fuzzy numbers, according to Remark 1, the problem FLP can also be written as follows:

$$
\begin{array}{cl}
\max & \widetilde{f}(\widetilde{\mathbf{a}}, \widetilde{\mathbf{x}}) \\
\text { subject to } & \sum_{\left\{j: j \in P_{i}\right\}} \widetilde{b}_{i j \alpha}^{L} \cdot \widetilde{x}_{j \alpha}^{L}+\sum_{\left\{j: j \in N_{i}\right\}} \widetilde{b}_{i j \alpha}^{L} \cdot \widetilde{x}_{j \alpha}^{U} \leq \widetilde{c}_{i \alpha}^{L} \text { for } i=1, \cdots, p \text { and } \alpha \in[0,1] ; \\
& \sum_{\left\{j: j \in N_{i}\right\}} \widetilde{b}_{i j \alpha}^{U} \cdot \widetilde{x}_{j \alpha}^{L}+\sum_{\left\{j: j \in P_{i}\right\}} \widetilde{b}_{i j \alpha}^{U} \cdot \widetilde{x}_{j \alpha}^{U} \leq \widetilde{c}_{i \alpha}^{U} \text { for } i=1, \cdots, p \text { and } \alpha \in[0,1] ; \\
& \widetilde{x}_{j \alpha}^{U} \geq \widetilde{x}_{j \alpha}^{L} \geq 0 \text { for } j=1, \cdots, q \text { and } \alpha \in[0,1] ; \\
& \text { each } \widetilde{x}_{j \alpha}^{L} \text { is an increasing function with respect to } \alpha \text { on }[0,1] \text { for } j=1, \cdots, q ; \\
& \text { each } \widetilde{x}_{j \alpha}^{U} \text { is a decreasing function with respect to } \alpha \text { on }[0,1] \text { for } j=1, \cdots, q .
\end{array}
$$

Given any two vectors $\mathbf{u}=\left(u_{1}, u_{2}\right)$ and $\mathbf{v}=\left(v_{1}, v_{2}\right)$ in $\mathbb{R}^{2}$. we define

$$
\mathbf{u} \leq \mathbf{v} \text { if and only if } u_{1} \leq v_{1} \text { and } u_{2} \leq v_{2},
$$

and

$$
\mathbf{u}<\mathbf{v} \text { if and only if } \mathbf{u} \leq \mathbf{v} \text { and } \mathbf{u} \neq \mathbf{v} \text {. }
$$

It is clear to see that $\left(u_{1}, u_{2}\right)<\left(v_{1}, v_{2}\right)$ if and only if

$$
\left\{\begin{array}{l}
u_{1}<v_{1} \\
u_{2} \leq v_{2}
\end{array}\right\} \text { or }\left\{\begin{array}{l}
u_{1} \leq v_{1} \\
u_{2}<v_{2}
\end{array}\right\} \text { or }\left\{\begin{array}{l}
u_{1}<v_{1} \\
u_{2}<v_{2}
\end{array}\right\} \text {. }
$$

Given a fuzzy number $\widetilde{a}$, we can consider the following vector

$$
\left(\int_{0}^{1} \widetilde{a}_{\alpha}^{L} d \alpha, \int_{0}^{1} \tilde{a}_{\alpha}^{U} d \alpha\right) \in \mathbb{R}^{2}
$$

Now, given any two fuzzy numbers $\widetilde{a}$ and $\widetilde{b}$, we define

$$
\widetilde{a} \preceq \widetilde{b} \text { if and only if }\left(\int_{0}^{1} \widetilde{a}_{\alpha}^{L} d \alpha, \int_{0}^{1} \widetilde{a}_{\alpha}^{U} d \alpha\right) \leq\left(\int_{0}^{1} \widetilde{b}_{\alpha}^{L} d \alpha, \int_{0}^{1} \widetilde{b}_{\alpha}^{U} d \alpha\right) .
$$

Equivalently, it means that

$$
\widetilde{a} \preceq \widetilde{b} \text { if and only if } \int_{0}^{1} \widetilde{a}_{\alpha}^{L} d \alpha \leq \int_{0}^{1} \widetilde{b}_{\alpha}^{L} d \alpha \text { and } \int_{0}^{1} \widetilde{a}_{\alpha}^{U} d \alpha \leq \int_{0}^{1} \widetilde{b}_{\alpha}^{U} d \alpha .
$$

We also define

$$
\widetilde{a} \prec \widetilde{b} \text { if and only if }\left(\int_{0}^{1} \widetilde{a}_{\alpha}^{L} d \alpha, \int_{0}^{1} \widetilde{a}_{\alpha}^{U} d \alpha\right)<\left(\int_{0}^{1} \widetilde{b}_{\alpha}^{L} d \alpha, \int_{0}^{1} \widetilde{b}_{\alpha}^{U} d \alpha\right) .
$$


It is clear to see that $\widetilde{a} \prec \widetilde{b}$ if and only if one of the following conditions is satisfied:

$$
\int_{0}^{1} \widetilde{a}_{\alpha}^{L} d \alpha<\int_{0}^{1} \widetilde{b}_{\alpha}^{L} d \alpha \text { and } \int_{0}^{1} \widetilde{a}_{\alpha}^{U} d \alpha \leq \int_{0}^{1} \widetilde{b}_{\alpha}^{U} d \alpha
$$

or

$$
\int_{0}^{1} \widetilde{a}_{\alpha}^{L} d \alpha \leq \int_{0}^{1} \widetilde{b}_{\alpha}^{L} d \alpha \text { and } \int_{0}^{1} \widetilde{a}_{\alpha}^{U} d \alpha<\int_{0}^{1} \widetilde{b}_{\alpha}^{U} d \alpha
$$

or

$$
\int_{0}^{1} \widetilde{a}_{\alpha}^{L} d \alpha<\int_{0}^{1} \widetilde{b}_{\alpha}^{L} d \alpha \text { and } \int_{0}^{1} \widetilde{a}_{\alpha}^{U} d \alpha<\int_{0}^{1} \widetilde{b}_{\alpha}^{U} d \alpha
$$

Definition 1. We say that $\widetilde{\mathbf{x}}^{*}=\left(\widetilde{x}_{1}^{*}, \cdots, \widetilde{x}_{q}^{*}\right)$ is a nondominated optimal solution of fuzzy optimization problem FLP if and only if there does not exists another feasible solution $\widetilde{\mathbf{x}}$ such that

$$
\widetilde{f}\left(\widetilde{\mathbf{a}}, \widetilde{\mathbf{x}}^{*}\right) \prec \widetilde{f}(\widetilde{\mathbf{a}}, \widetilde{\mathbf{x}}),
$$

where $\widetilde{f}(\widetilde{\mathbf{a}}, \widetilde{\mathbf{x}})$ denotes the fuzzy objective function of FLP.

From (7), we see that $\widetilde{\mathbf{x}}^{*}$ is a nondominated optimal solution of problem FLP if and only if there does not exists another feasible solution $\widetilde{\mathbf{x}}$ such that

$$
\left(\int_{0}^{1}\left(\widetilde{f}\left(\widetilde{\mathbf{a}}, \widetilde{\mathbf{x}}^{*}\right)\right)_{\alpha}^{L} d \alpha, \int_{0}^{1}\left(\widetilde{f}\left(\widetilde{\mathbf{a}}, \widetilde{\mathbf{x}}^{*}\right)\right)_{\alpha}^{U} d \alpha\right)<\left(\int_{0}^{1}(\widetilde{f}(\widetilde{\mathbf{a}}, \widetilde{\mathbf{x}}))_{\alpha}^{L} d \alpha, \int_{0}^{1}(\widetilde{f}(\widetilde{\mathbf{a}}, \widetilde{\mathbf{x}}))_{\alpha}^{U} d \alpha\right) .
$$

We define

$$
f^{L}(\widetilde{\mathbf{a}}, \widetilde{\mathbf{x}})=\int_{0}^{1}(\widetilde{f}(\widetilde{\mathbf{a}}, \widetilde{\mathbf{x}}))_{\alpha}^{L} d \alpha \text { and } f^{U}(\widetilde{\mathbf{a}}, \widetilde{\mathbf{x}})=\int_{0}^{1}(\widetilde{f}(\widetilde{\mathbf{a}}, \widetilde{\mathbf{x}}))_{\alpha}^{U} d \alpha
$$

and consider the following biobjective optimization problem

$$
\begin{aligned}
\text { (BOP) } \max \quad & \left(f^{L}(\widetilde{\mathbf{a}}, \widetilde{\mathbf{x}}), f^{U}(\widetilde{\mathbf{a}}, \widetilde{\mathbf{x}})\right) \\
\text { subject to } & \sum_{\left\{j: j \in P_{i}\right\}} \widetilde{b}_{i j \alpha}^{L} \cdot \widetilde{x}_{j \alpha}^{L}+\sum_{\left\{j: j \in N_{i}\right\}} \widetilde{b}_{i j \alpha}^{L} \cdot \widetilde{x}_{j \alpha}^{U} \leq \widetilde{c}_{i \alpha}^{L} \text { for } i=1, \cdots, p \text { and } \alpha \in[0,1] ; \\
& \sum_{\left\{j: j \in N_{i}\right\}} \widetilde{b}_{i j \alpha}^{U} \cdot \widetilde{x}_{j \alpha}^{L}+\sum_{\left\{j: j \in P_{i}\right\}} \widetilde{b}_{i j \alpha}^{U} \cdot \widetilde{x}_{j \alpha}^{U} \leq \widetilde{c}_{i \alpha}^{U} \text { for } i=1, \cdots, p \text { and } \alpha \in[0,1] ; \\
& 0 \leq \widetilde{x}_{j \alpha}^{L} \leq \widetilde{x}_{j \alpha}^{U} \text { for } j=1, \cdots, q \text { and } \alpha \in[0,1] ; \\
& \text { each } \widetilde{x}_{j \alpha}^{L} \text { is an increasing function with respect to } \alpha \text { on }[0,1] \text { for } j=1, \cdots, q ; \\
& \text { each } \widetilde{x}_{j \alpha}^{U} \text { is a decreasing function with respect to } \alpha \text { on }[0,1] \text { for } j=1, \cdots, q .
\end{aligned}
$$

Recall that $\widetilde{\mathbf{x}}^{*}=\left(\widetilde{x}_{1}^{*}, \cdots, \widetilde{x}_{q}^{*}\right)$ is a Pareto optimal solution of the biobjective optimization problem BOP if and only if there does not exists another feasible solution $\widetilde{\mathbf{x}}$ such that

$$
\left(f^{L}(\widetilde{\mathbf{a}}, \widetilde{\mathbf{x}}), f^{U}(\widetilde{\mathbf{a}}, \widetilde{\mathbf{x}})\right)<\left(f^{L}\left(\widetilde{\mathbf{a}}, \widetilde{\mathbf{x}}^{*}\right), f^{U}\left(\widetilde{\mathbf{a}}, \widetilde{\mathbf{x}}^{*}\right)\right) .
$$

It is clear to see that the expressions (8) and (9) are equivalent. Therefore we have the following useful result.

Proposition 1. $\widetilde{\mathbf{x}}^{*}$ is a nondominated optimal solution of FLP problem if and only if $\widetilde{\mathbf{x}}^{*}$ is a Pareto optimal solution of BOP. 
Now we consider the following scalar optimization problem corresponding to the BOP:

$$
\begin{array}{cl}
\max & f^{L}(\widetilde{\mathbf{a}}, \widetilde{\mathbf{x}})+f^{U}(\widetilde{\mathbf{a}}, \widetilde{\mathbf{x}}) \\
\text { subject to } & \sum_{\left\{j: j \in P_{i}\right\}} \widetilde{b}_{i j \alpha}^{L} \cdot \widetilde{x}_{j \alpha}^{L}+\sum_{\left\{j: j \in N_{i}\right\}} \widetilde{b}_{i j \alpha}^{L} \cdot \widetilde{x}_{j \alpha}^{U} \leq \widetilde{c}_{i \alpha}^{L} \text { for } i=1, \cdots, p \text { and } \alpha \in[0,1] ; \\
& \sum_{\left\{j: j \in N_{i}\right\}} \widetilde{b}_{i j \alpha}^{U} \cdot \widetilde{x}_{j \alpha}^{L}+\sum_{\left\{j: j \in P_{i}\right\}} \widetilde{b}_{i j \alpha}^{U} \cdot \widetilde{x}_{j \alpha}^{U} \leq \widetilde{c}_{i \alpha}^{U} \text { for } i=1, \cdots, p \text { and } \alpha \in[0,1] ; \\
& 0 \leq \widetilde{x}_{j \alpha}^{L} \leq \widetilde{x}_{j \alpha}^{U} \text { for } j=1, \cdots, q \text { and } \alpha \in[0,1] ; \\
& \text { each } \widetilde{x}_{j \alpha}^{L} \text { is an increasing function with respect to } \alpha \text { on }[0,1] \text { for } j=1, \cdots, q ; \\
& \text { each } \widetilde{x}_{j \alpha}^{U} \text { is a decreasing function with respect to } \alpha \text { on }[0,1] \text { for } j=1, \cdots, q .
\end{array}
$$

Then we have the following interesting result.

Proposition 2. If $\widetilde{\mathbf{x}}^{*}$ is an optimal solution of scalar optimization problem (SOP), then $\widetilde{\mathbf{x}}^{*}$ is also a Pareto optimal solution of BOP.

Proof. Suppose that $\widetilde{\mathbf{x}}^{*}$ is not a Pareto optimal solution of the BOP problem. Then there exists $\widetilde{\mathbf{x}}^{\circ}$ such that

$$
\left(f^{L}\left(\widetilde{\mathbf{a}}, \widetilde{\mathbf{x}}^{\circ}\right), f^{U}\left(\widetilde{\mathbf{a}}, \widetilde{\mathbf{x}}^{\circ}\right)\right)<\left(f^{L}\left(\widetilde{\mathbf{a}}, \widetilde{\mathbf{x}}^{*}\right), f^{U}\left(\widetilde{\mathbf{a}}, \widetilde{\mathbf{x}}^{*}\right)\right) .
$$

Using (6), we see that

$$
f^{L}\left(\widetilde{\mathbf{a}}, \widetilde{\mathbf{x}}^{\circ}\right)+f^{U}\left(\widetilde{\mathbf{a}}, \widetilde{\mathbf{x}}^{\circ}\right)<f^{L}\left(\widetilde{\mathbf{a}}, \widetilde{\mathbf{x}}^{*}\right)+f^{U}\left(\widetilde{\mathbf{a}}, \widetilde{\mathbf{x}}^{*}\right),
$$

which contradicts the optimality of $\widetilde{\mathbf{x}}^{*}$ for the SOP problem. This completes the proof.

Theorem 1. If $\widetilde{\mathbf{x}}$ is an optimal solution of SOP, then $\widetilde{\mathbf{x}}$ is also a nondominated optimal solution of FOP problem.

Proof. The result follows immediately from Propositions 1 and 2.

From Theorem 1, in order to obtain the nondominated optimal solution of the original FOP, it suffices to find the optimal solution of SOP. In the sequel, we are going to numerically solve the SOP by presenting the error estimation.

We assume that each $\widetilde{a}_{j}$ is a nonnegative or nonpositive fuzzy number for $j=1, \cdots, q$, and define the sets of indices as follows:

$$
P=\left\{j: \widetilde{a}_{j} \text { is a nonnegative fuzzy number }\right\}
$$

and

$$
N=\left\{j: \widetilde{a}_{j} \text { is a nonpositive fuzzy number }\right\} .
$$

It is clear to see that $P \cup N=\{1,2, \cdots, q\}$. Let us recall that we also have the sets of indices $P_{i}$ and $N_{i}$ defined in (4) and (5), respectively, for $i=1, \cdots, p$.

Using (1)-(3), we have

$$
(\widetilde{f}(\widetilde{\mathbf{a}}, \widetilde{\mathbf{x}}))_{\alpha}^{L}=\widetilde{a}_{1 \alpha}^{L} \cdot \widetilde{x}_{1 \alpha}^{L}+\widetilde{a}_{2 \alpha}^{L} \cdot \widetilde{x}_{2 \alpha}^{L}+\cdots+\widetilde{a}_{q \alpha}^{L} \cdot \widetilde{x}_{q \alpha}^{L}=\sum_{j \in P} \widetilde{a}_{j \alpha}^{L} \cdot \widetilde{x}_{j \alpha}^{L}+\sum_{j \in N} \widetilde{a}_{j \alpha}^{L} \cdot \widetilde{x}_{j \alpha}^{U}
$$

and

$$
(\widetilde{f}(\widetilde{\mathbf{a}}, \widetilde{\mathbf{x}}))_{\alpha}^{U}=\widetilde{a}_{1 \alpha}^{U} \cdot \widetilde{x}_{1 \alpha}^{U}+\widetilde{a}_{2 \alpha}^{U} \cdot \widetilde{x}_{2 \alpha}^{U}+\cdots+\widetilde{a}_{q \alpha}^{U} \cdot \widetilde{x}_{q \alpha}^{U}=\sum_{j \in N} \widetilde{a}_{j \alpha}^{U} \cdot \widetilde{x}_{j \alpha}^{L}+\sum_{j \in P} \widetilde{a}_{j \alpha}^{U} \cdot \widetilde{x}_{j \alpha}^{U} .
$$


Then the SOP can be rewritten as follows:

(SOP) $\max \sum_{j \in P}\left(\int_{0}^{1} \widetilde{a}_{j \alpha}^{L} \cdot \widetilde{x}_{j \alpha}^{L} d \alpha+\int_{0}^{1} \widetilde{a}_{j \alpha}^{U} \cdot \widetilde{x}_{j \alpha}^{U} d \alpha\right)+\sum_{j \in N}\left(\int_{0}^{1} \widetilde{a}_{j \alpha}^{L} \cdot \widetilde{x}_{j \alpha}^{U} d \alpha+\int_{0}^{1} \widetilde{a}_{j \alpha}^{U} \cdot \widetilde{x}_{j \alpha}^{L} d \alpha\right)$

subject to $\sum_{\left\{j: j \in P_{i}\right\}} \widetilde{b}_{i j \alpha}^{L} \cdot \widetilde{x}_{j \alpha}^{L}+\sum_{\left\{j: j \in N_{i}\right\}} \widetilde{b}_{i j \alpha}^{L} \cdot \widetilde{x}_{j \alpha}^{U} \leq \widetilde{c}_{i \alpha}^{L}$ for $i=1, \cdots, p$ and $\alpha \in[0,1]$;

$\sum_{\left\{j: j \in N_{i}\right\}} \widetilde{b}_{i j \alpha}^{U} \cdot \widetilde{x}_{j \alpha}^{L}+\sum_{\left\{j: j \in P_{i}\right\}} \widetilde{b}_{i j \alpha}^{U} \cdot \widetilde{x}_{j \alpha}^{U} \leq \widetilde{c}_{i \alpha}^{U}$ for $i=1, \cdots, p$ and $\alpha \in[0,1] ;$

$0 \leq \widetilde{x}_{j \alpha}^{L} \leq \widetilde{x}_{j \alpha}^{U}$ for $j=1, \cdots, q$ and $\alpha \in[0,1] ;$

each $\widetilde{x}_{j \alpha}^{L}$ is an increasing function with respect to $\alpha$ on $[0,1]$ for $j=1, \cdots, q$;

each $\widetilde{x}_{j \alpha}^{U}$ is a decreasing function with respect to $\alpha$ on $[0,1]$ for $j=1, \cdots, q$.

For $j=1, \cdots, q$, we define the real-valued functions $a_{j}^{L}, a_{j}^{U}, x_{j}^{L}$ and $x_{j}^{U}$ on $[0,1]$ as follows:

$$
a_{j}^{L}(t)=\widetilde{a}_{j t}^{L} \text { and } a_{j}^{U}(t)=\widetilde{a}_{j t}^{U}
$$

and

$$
x_{j}^{L}(t)=\widetilde{x}_{j t}^{L} \text { and } x_{j}^{U}(t)=\widetilde{x}_{j t}^{U} .
$$

For $i=1, \cdots, p$, we define the real-valued functions $c_{i}^{L}$ and $c_{i}^{U}$ on $[0,1]$ as follows:

$$
c_{i}^{L}(t)=\widetilde{c}_{i t}^{L} \text { and } c_{i}^{U}(t)=\widetilde{c}_{i t}^{U} .
$$

For $i=1, \cdots, p$ and $j=1, \cdots, q$, we define the real-valued functions $b_{i j}^{L}$ and $b_{i j}^{U}$ on $[0,1]$ as follows:

$$
b_{i j}^{L}(t)=\widetilde{b}_{i j t}^{L} \text { and } b_{i j}^{U}(t)=\widetilde{b}_{i j t}^{U} .
$$

Then the SOP can be written as follows:

$$
\begin{array}{ll}
\text { (SOP) } \quad \max \quad \sum_{j \in P}\left(\int_{0}^{1} a_{j}^{L}(t) \cdot x_{j}^{L}(t) d t+\int_{0}^{1} a_{j}^{U}(t) \cdot x_{j}^{U}(t) d t\right) \\
& +\sum_{j \in N}\left(\int_{0}^{1} a_{j}^{L}(t) \cdot x_{j}^{U}(t) d t+\int_{0}^{1} a_{j}^{U}(t) \cdot x_{j}^{L}(t) d t\right) \\
\text { subject to } \quad & \sum_{\left\{j: j \in P_{i}\right\}} b_{i j}^{L}(t) \cdot x_{j}^{L}(t)+\sum_{\left\{j: j \in N_{i}\right\}} b_{i j}^{L}(t) \cdot x_{j}^{U}(t) \leq c_{i}^{L}(t) \\
& \text { for } t \in[0,1] \text { and } i=1, \cdots, p ; \\
& \sum_{\left\{j: j \in N_{i}\right\}} b_{i j}^{U}(t) \cdot x_{j}^{L}(t)+\sum_{\left\{j: j \in P_{i}\right\}} b_{i j}^{U}(t) \cdot x_{j}^{U}(t) \leq c_{i}^{U}(t) \\
& \text { for } t \in[0,1] \text { and } i=1, \cdots, p ; \\
& 0 \leq x_{j}^{L}(t) \leq x_{j}^{U}(t) \text { for } j=1, \cdots, q \text { and } t \in[0,1] ; \\
& \text { each } x_{j}^{L}(t) \text { is an increasing function on }[0,1] \text { for } j=1, \cdots, q ; \\
& \text { each } x_{j}^{U}(t) \text { is a decreasing function on }[0,1] \text { for } j=1, \cdots, q .
\end{array}
$$

We note that the "decision variables" in problem SOP are monotonic real-valued functions defined on $[0,1]$, which can be regarded as the decision functions of problem SOP.

In order to design the computational procedure, we assume that the following two conditions are satisfied:

- $\quad$ each $\widetilde{c}_{i}$ is assumed to be a nonnegative fuzzy number for $i=1, \ldots$, $p$; 
- we assume that

$$
\sigma^{P N}=\min _{j=1, \cdots, q}\left\{\sum_{\left\{i: j \in P_{i}\right\}} \inf _{t \in[0,1]} b_{i j}^{L}(t)+\sum_{\left\{i: j \in N_{i}\right\}} \inf _{t \in[0,1]} b_{i j}^{U}(t)\right\}>0,
$$

and

$$
\sigma^{N P}=\min _{j=1, \cdots, q}\left\{\sum_{\left\{i: j \in N_{i}\right\}} \inf _{t \in[0,1]} b_{i j}^{L}(t)+\sum_{\left\{i: j \in P_{i}\right\}} \inf _{t \in[0,1]} b_{i j}^{U}(t)\right\}>0 .
$$

Since $b_{i j}^{L}$ is increasing and $b_{i j}^{U}$ is decreasing on $[0,1]$, if $b_{i j}^{L}$ and $b_{i j}^{U}$ are also continuous on $[0,1]$, then

$$
\sigma^{P N}=\min _{j=1, \cdots, q}\left\{\sum_{\left\{i: j \in P_{i}\right\}} b_{i j}^{L}(0)+\sum_{\left\{i: j \in N_{i}\right\}} b_{i j}^{U}(1)\right\},
$$

and

$$
\sigma^{N P}=\min _{j=1, \cdots, q}\left\{\sum_{\left\{i: j \in N_{i}\right\}} b_{i j}^{L}(0)+\sum_{\left\{i: j \in P_{i}\right\}} b_{i j}^{U}(1)\right\} .
$$

Given any fixed $\delta$ with $0<\delta<1$, we define the following auxiliary problem of the SOP by considering the monotonic properties of decision functions $x_{j}^{L}$ and $x_{j}^{U}$ for $j=1, \cdots, q$ :

$$
\begin{array}{ll}
\left(\mathrm{SOP}_{\delta}\right) \quad \max \quad \sum_{j \in P}\left(\int_{0}^{1} a_{j}^{L}(t) \cdot x_{j}^{L}(t) d \alpha+\int_{0}^{1} a_{j}^{U}(t) \cdot x_{j}^{U}(t) d \alpha\right) \\
& +\sum_{j \in N}\left(\int_{0}^{1} a_{j}^{L}(t) \cdot x_{j}^{U}(t) d \alpha+\int_{0}^{1} a_{j}^{U}(t) \cdot x_{j}^{L}(t) d \alpha\right) \\
\text { subject to } \quad \sum_{\left\{j: j \in P_{i}\right\}} b_{i j}^{L}(t) \cdot x_{j}^{L}(t)+\sum_{\left\{j: j \in N_{i}\right\}} b_{i j}^{L}(t) \cdot x_{j}^{U}(t) \leq c_{i}^{L}(t) \\
\quad \text { for } t \in[0,1] \text { and } i=1, \cdots, p ; \\
\sum_{\left\{j: j \in N_{i}\right\}} b_{i j}^{U}(t) \cdot x_{j}^{L}(t)+\sum_{\left\{j: j \in P_{i}\right\}} b_{i j}^{U}(t) \cdot x_{j}^{U}(t) \leq c_{i}^{U}(t) \\
\quad \text { for } t \in[0,1] \text { and } i=1, \cdots, p ; \\
0 \leq x_{j}^{L}(t) \leq x_{j}^{U}(t) \text { for } j=1, \cdots, q \text { and } t \in[0,1] ; \\
x_{j}^{L}(t) \leq x_{j}^{L}(t+\delta) \text { for } t \in[0,1-\delta] \text { and } j=1, \cdots, q ; \\
x_{j}^{U}(t+\delta) \leq x_{j}^{U}(t) \text { for } t \in[0,1-\delta] \text { and } j=1, \cdots, q .
\end{array}
$$

Remark 2. It is clear to see that each feasible solution of problem SOP is also a feasible solution of problem $\left(\mathrm{SOP}_{\delta}\right)$.

In order to investigate the error estimation, we need to consider the dual problem of of problem $\left(\mathrm{SOP}_{\delta}\right)$, which is defined as follows:

$$
\begin{gathered}
\left(\operatorname{DSOP}_{\delta}\right) \quad \min \sum_{i=1}^{p} \int_{0}^{1} c_{i}^{L}(t) \cdot y_{i}^{I}(t) d t+\sum_{i=1}^{p} \int_{0}^{1} c_{i}^{U}(t) \cdot y_{i}^{I I}(t) d t \\
\text { subject to } \sum_{\left\{i: j \in P_{i}\right\}} b_{i j}^{L}(t) \cdot y_{i}^{I}(t)+\sum_{\left\{i: j \in N_{i}\right\}} b_{i j}^{U}(t) \cdot y_{i}^{I I}(t)+y_{j}^{I I I}(t)+y_{j}^{I V}(t) \geq a_{j}^{L}(t) \\
\quad \text { for } t \in[0, \delta) \text { and } j \in P ;
\end{gathered}
$$




$$
\begin{aligned}
& \sum_{\left\{i: j \in P_{i}\right\}} b_{i j}^{L}(t) \cdot y_{i}^{I}(t)+\sum_{\left\{i: j \in N_{i}\right\}} b_{i j}^{U}(t) \cdot y_{i}^{I I}(t)+y_{j}^{I I I}(t)+y_{j}^{I V}(t) \geq a_{j}^{U}(t) \\
& \quad \text { for } t \in[0, \delta) \text { and } j \in N ;
\end{aligned}
$$$$
\sum_{\left\{i: j \in N_{i}\right\}} b_{i j}^{L}(t) \cdot y_{i}^{I}(t)+\sum_{\left\{i: j \in P_{i}\right\}} b_{i j}^{U}(t) \cdot y_{i}^{I I}(t)-y_{j}^{I I I}(t)-y_{j}^{V}(t) \geq a_{j}^{L}(t)
$$$$
\text { for } t \in[0, \delta) \text { and } j \in N \text {; }
$$

$\sum_{\left\{i: j \in N_{i}\right\}} b_{i j}^{L}(t) \cdot y_{i}^{I}(t)+\sum_{\left\{i: j \in P_{i}\right\}} b_{i j}^{U}(t) \cdot y_{i}^{I I}(t)-y_{j}^{I I I}(t)-y_{j}^{V}(t) \geq a_{j}^{U}(t)$

for $t \in[0, \delta)$ and $j \in P$;

$$
\begin{array}{r}
\sum_{\left\{i: j \in P_{i}\right\}} b_{i j}^{L}(t) \cdot y_{i}^{I}(t)+\sum_{\left\{i: j \in N_{i}\right\}} b_{i j}^{U}(t) \cdot y_{i}^{I I}(t)+y_{j}^{I I I}(t)+y_{j}^{I V}(t) \\
-y_{j}^{I V}(t-\delta) \geq a_{j}^{L}(t) \text { for } t \in[\delta, 1-\delta) \text { and } j \in P ; \\
\sum_{\left\{i: j \in P_{i}\right\}} b_{i j}^{L}(t) \cdot y_{i}^{I}(t)+\sum_{\left\{i: j \in N_{i}\right\}} b_{i j}^{U}(t) \cdot y_{i}^{I I}(t)+y_{j}^{I I I}(t)+y_{j}^{I V}(t) \\
-y_{j}^{I V}(t-\delta) \geq a_{j}^{U}(t) \text { for } t \in[\delta, 1-\delta) \text { and } j \in N ;
\end{array}
$$

$$
\begin{aligned}
\sum_{\left\{i: j \in N_{i}\right\}} & b_{i j}^{L}(t) \cdot y_{i}^{I}(t)+\sum_{\left\{i: j \in P_{i}\right\}} b_{i j}^{U}(t) \cdot y_{i}^{I I}(t)-y_{j}^{I I I}(t) \\
& -y_{j}^{V}(t)+y_{j}^{V}(t-\delta) \geq a_{j}^{L}(t) \text { for } t \in[\delta, 1-\delta) \text { and } j \in N
\end{aligned}
$$

$$
\begin{aligned}
\sum_{\left\{i: j \in N_{i}\right\}} & b_{i j}^{L}(t) \cdot y_{i}^{I}(t)+\sum_{\left\{i: j \in P_{i}\right\}} b_{i j}^{U}(t) \cdot y_{i}^{I I}(t)-y_{j}^{I I I}(t) \\
& -y_{j}^{V}(t)+y_{j}^{V}(t-\delta) \geq a_{j}^{U}(t) \text { for } t \in[\delta, 1-\delta) \text { and } j \in P ;
\end{aligned}
$$

$$
\begin{aligned}
& \sum_{\left\{i: j \in P_{i}\right\}} b_{i j}^{L}(t) \cdot y_{i}^{I}(t)+\sum_{\left\{i: j \in N_{i}\right\}} b_{i j}^{U}(t) \cdot y_{i}^{I I}(t)+y_{j}^{I I I}(t)-y_{j}^{I V}(t-\delta) \geq a_{j}^{L}(t) \\
& \quad \text { for } t \in[1-\delta, 1] \text { and } j \in P ;
\end{aligned}
$$

$$
\begin{aligned}
& \sum_{\left\{i: j \in P_{i}\right\}} b_{i j}^{L}(t) \cdot y_{i}^{I}(t)+\sum_{\left\{i: j \in N_{i}\right\}} b_{i j}^{U}(t) \cdot y_{i}^{I I}(t)+y_{j}^{I I I}(t)-y_{j}^{I V}(t-\delta) \geq a_{j}^{U}(t) \\
& \quad \text { for } t \in[1-\delta, 1] \text { and } j \in N ; \\
& \sum_{\left\{i: j \in N_{i}\right\}} b_{i j}^{L}(t) \cdot y_{i}^{I}(t)+\sum_{\left\{i: j \in P_{i}\right\}} b_{i j}^{U}(t) \cdot y_{i}^{I I}(t)-y_{j}^{I I}(t)+y_{j}^{V}(t-\delta) \geq a_{j}^{L}(t) \\
& \quad \text { for } t \in[1-\delta, 1] \text { and } j \in N ;
\end{aligned}
$$




$$
\begin{aligned}
& \sum_{\left\{i: j \in N_{i}\right\}} b_{i j}^{L}(t) \cdot y_{i}^{I}(t)+\sum_{\left\{i: j \in P_{i}\right\}} b_{i j}^{U}(t) \cdot y_{i}^{I I}(t)-y_{j}^{I I I}(t)+y_{j}^{V}(t-\delta) \geq a_{j}^{U}(t) \\
& \quad \text { for } t \in[1-\delta, 1] \text { and } j \in P ; \\
& y_{i}^{I}(t), y_{i}^{I I}(t) \geq 0 \text { for } t \in[0,1] \text { and } i=1, \cdots, p ; \\
& y_{j}^{I I I}(t), y_{j}^{I V}(t), y_{j}^{V}(t) \geq 0 \text { for } t \in[0,1] \text { and } j=1, \cdots, q .
\end{aligned}
$$

The primal and dual pair of problems $\left(\mathrm{SOP}_{\delta}\right)$ and $\left(\mathrm{DSOP}_{\delta}\right)$ are really helpful for further discussion. The reason for naming them as the primal and dual pair of problems is that we can establish the weak duality theorem shown in Theorem 2 and the asymptotic no duality gap shown in Theorem 3 below.

Given an optimization problem $(\mathrm{P})$, if problem $(\mathrm{P})$ is a maximization problem, then $V(\mathrm{P})$ denotes the supremum of its objective function, and if problem $(\mathrm{P})$ is a minimization problem, then $V(\mathrm{P})$ denotes the infimum of its objective function. We have to mention that the supremum or infimum is attained when the optimal solution of problem $(\mathrm{P})$ exists. In other words, $V(\mathrm{P})$ denotes the optimal objective value of problem $(\mathrm{P})$ when there exists a feasible solution $x^{*}$ of problem $(\mathrm{P})$ such that the supremum or infimum is equal to the objective value of $x^{*}$.

We first prove the weak duality theorem $V\left(\mathrm{SOP}_{\delta}\right) \leq V\left(\mathrm{DSOP}_{\delta}\right)$. The asymptotic strong duality theorem can also be obtained in the subsequent discussion by showing that

$$
\limsup _{n \rightarrow \infty} V\left(\mathrm{SOP}_{1 / n}\right)=\limsup _{n \rightarrow \infty} V\left(\mathrm{DSOP}_{1 / n}\right),
$$

where $\delta$ is taken to be $1 / n$ for $n \in \mathbb{N}$. The weak duality theorem is also useful for investigating the error estimation.

Theorem 2. (Weak Duality Theorem) Considering the primal and dual pair of problems $\left(\mathrm{SOP}_{\delta}\right)$ and $\left(\mathrm{DSOP}_{\delta}\right)$, respectively, for any feasible solution $\left(\mathbf{x}^{L}, \mathbf{x}^{U}\right)$ of primal problem SOP and any feasible solution $\left(\mathbf{y}^{I}, \mathbf{y}^{I I}, \mathbf{y}^{I I I}, \mathbf{y}^{I V}, \mathbf{y}^{V}\right)$ of dual problem $\left(\mathrm{DSOP}_{\delta}\right)$, we have

$$
\begin{aligned}
& \sum_{j \in P}\left(\int_{0}^{1} a_{j}^{L}(t) \cdot x_{j}^{L}(t) d \alpha+\int_{0}^{1} a_{j}^{U}(t) \cdot x_{j}^{U}(t) d \alpha\right)+\sum_{j \in N}\left(\int_{0}^{1} a_{j}^{L}(t) \cdot x_{j}^{U}(t) d \alpha+\int_{0}^{1} a_{j}^{U}(t) \cdot x_{j}^{L}(t) d \alpha\right) \\
& \quad \leq \sum_{i=1}^{p} \int_{0}^{1} c_{i}^{L}(t) \cdot y_{i}^{I}(t) d t+\sum_{i=1}^{p} \int_{0}^{1} c_{i}^{U}(t) \cdot y_{i}^{I I}(t) d t .
\end{aligned}
$$

Moreover, we also have

$$
V(\mathrm{SOP}) \leq V\left(\mathrm{SOP}_{\delta}\right) \leq V\left(\mathrm{DSOP}_{\delta}\right)
$$

Proof. We first note that $\left(\mathbf{x}^{L}, \mathbf{x}^{U}\right)$ is also a feasible solution of problem $\left(\mathrm{SOP}_{\delta}\right)$ by Remark 2. Since all the decision functions of problems $\left(\mathrm{SOP}_{\delta}\right)$ and $\left(\mathrm{DSOP}_{\delta}\right)$ are nonnegative, we have

$$
\sum_{j \in P} \int_{0}^{1} a_{j}^{L}(t) \cdot x_{j}^{L}(t) d t=\sum_{j \in P}\left[\int_{0}^{\delta} a_{j}^{L}(t) \cdot x_{j}^{L}(t) d t+\int_{\delta}^{1-\delta} a_{j}^{L}(t) \cdot x_{j}^{L}(t) d t+\int_{1-\delta}^{1} a_{j}^{L}(t) \cdot x_{j}^{L}(t) d t\right]
$$




$$
\begin{aligned}
\leq & \sum_{j \in P} \int_{0}^{\delta}\left[\sum_{\left\{i: j \in P_{i}\right\}} b_{i j}^{L}(t) \cdot y_{i}^{I}(t)+\sum_{\left\{i: j \in N_{i}\right\}} b_{i j}^{U}(t) \cdot y_{i}^{I I}(t)+y_{j}^{I I I}(t)+y_{j}^{I V}(t)\right] \cdot x_{j}^{L}(t) d t \\
+ & \sum_{j \in P} \int_{\delta}^{1-\delta}\left[\sum_{\left\{i: j \in P_{i}\right\}} b_{i j}^{L}(t) \cdot y_{i}^{I}(t)\right. \\
& \left.+\sum_{\left\{i: j \in N_{i}\right\}} b_{i j}^{U}(t) \cdot y_{i}^{I I}(t)+y_{j}^{I I I}(t)+y_{j}^{I V}(t)-y_{j}^{I V}(t-\delta)\right] \cdot x_{j}^{L}(t) d t \\
+ & \sum_{j \in P} \int_{1-\delta}^{1}\left[\sum_{\left\{i: j \in P_{i}\right\}} b_{i j}^{L}(t) \cdot y_{i}^{I}(t)+\sum_{\left\{i: j \in N_{i}\right\}} b_{i j}^{U}(t) \cdot y_{i}^{I I}(t)+y_{j}^{I I I}(t)-y_{j}^{I V}(t-\delta)\right] \cdot x_{j}^{L}(t) d t
\end{aligned}
$$

(using the constraints (19), (23) and (27) of dual problem $\left(\mathrm{DSOP}_{\delta}\right)$ )

$$
\begin{aligned}
= & \sum_{i=1}^{p} \int_{0}^{1}\left(\sum_{\left\{j: j \in P_{i} \cap P\right\}} b_{i j}^{L}(t) \cdot x_{j}^{L}(t)\right) \cdot y_{i}^{I}(t) d t+\sum_{i=1}^{p} \int_{0}^{1}\left(\sum_{\left\{j: j \in N_{i} \cap P\right\}} b_{i j}^{U}(t) \cdot x_{j}^{L}(t)\right) \cdot y_{i}^{I I}(t) d t \\
& +\sum_{j \in P} \int_{0}^{1} y_{j}^{I I I}(t) \cdot x_{j}^{L}(t) d t+\sum_{j \in P} \int_{0}^{1-\delta} y_{j}^{I V}(t) \cdot x_{j}^{L}(t) d t-\sum_{j \in P} \int_{\delta}^{1} y_{j}^{I V}(t-\delta) \cdot x_{j}^{L}(t) d t \\
= & \sum_{i=1}^{p} \int_{0}^{1}\left(\sum_{\left\{j: j \in P_{i} \cap P\right\}} b_{i j}^{L}(t) \cdot x_{j}^{L}(t)\right) \cdot y_{i}^{I}(t) d t+\sum_{i=1}^{p} \int_{0}^{1}\left(\sum_{\left\{j: j \in N_{i} \cap P\right\}} b_{i j}^{U}(t) \cdot x_{j}^{L}(t)\right) \cdot y_{i}^{I I}(t) d t \\
& +\sum_{j \in P} \int_{0}^{1} y_{j}^{I I I}(t) \cdot x_{j}^{L}(t) d t+\sum_{j \in P} \int_{0}^{1-\delta} y_{j}^{I V}(t) \cdot\left[x_{j}^{L}(t)-x_{j}^{L}(t+\delta)\right] d t,
\end{aligned}
$$

and

$$
\begin{aligned}
& \sum_{j \in N} \int_{0}^{1} a_{j}^{L}(t) \cdot x_{j}^{U}(t) d t=\sum_{j \in N}\left[\int_{0}^{\delta} a_{j}^{L}(t) \cdot x_{j}^{U}(t) d t+\int_{\delta}^{1-\delta} a_{j}^{L}(t) \cdot x_{j}^{U}(t) d t+\int_{1-\delta}^{1} a_{j}^{L}(t) \cdot x_{j}^{U}(t) d t\right] \\
& \leq \sum_{j \in N} \int_{0}^{\delta}\left[\sum_{\left\{i: j \in N_{i}\right\}} b_{i j}^{L}(t) \cdot y_{i}^{I}(t)+\sum_{\left\{i: j \in P_{i}\right\}} b_{i j}^{U}(t) \cdot y_{i}^{I I}(t)-y_{j}^{I I I}(t)-y_{j}^{V}(t)\right] \cdot x_{j}^{U}(t) d t \\
& +\sum_{j \in N} \int_{\delta}^{1-\delta}\left[\sum_{\left\{i: j \in N_{i}\right\}} b_{i j}^{L}(t) \cdot y_{i}^{I}(t)\right. \\
& \left.\quad+\sum_{\left\{i: j \in P_{i}\right\}} b_{i j}^{U}(t) \cdot y_{i}^{I I}(t)-y_{j}^{I I I}(t)-y_{j}^{V}(t)+y_{j}^{V}(t-\delta)\right] \cdot x_{j}^{U}(t) d t \\
& +\sum_{j \in N} \int_{1-\delta}^{1}\left[\sum_{\left\{i: j \in N_{i}\right\}} b_{i j}^{L}(t) \cdot y_{i}^{I}(t)+\sum_{\left\{i: j \in P_{i}\right\}} b_{i j}^{U}(t) \cdot y_{i}^{I I}(t)-y_{j}^{I I I}(t)+y_{j}^{V}(t-\delta)\right] \cdot x_{j}^{U}(t) d t
\end{aligned}
$$

(using the constraints (21), (25) and (29) of dual problem $\left(\mathrm{DSOP}_{\delta}\right)$ ) 


$$
\begin{aligned}
= & \sum_{i=1}^{p} \int_{0}^{1}\left(\sum_{\left\{j: j \in N_{i} \cap N\right\}} b_{i j}^{L}(t) \cdot x_{j}^{U}(t)\right) \cdot y_{i}^{I}(t) d t+\sum_{i=1}^{p} \int_{0}^{1}\left(\sum_{\left\{j: j \in P_{i} \cap N\right\}} b_{i j}^{U}(t) \cdot x_{j}^{U}(t)\right) \cdot y_{i}^{I I}(t) d t \\
& -\sum_{j \in N} \int_{0}^{1} y_{j}^{I I I}(t) \cdot x_{j}^{U}(t) d t-\sum_{j \in N} \int_{0}^{1-\delta} y_{j}^{V}(t) \cdot x_{j}^{U}(t) d t+\sum_{j \in N} \int_{\delta}^{1} y_{j}^{V}(t-\delta) \cdot x_{j}^{U}(t) d t \\
= & \sum_{i=1}^{p} \int_{0}^{1}\left(\sum_{\left\{j: j \in N_{i} \cap N\right\}} b_{i j}^{L}(t) \cdot x_{j}^{U}(t)\right) \cdot y_{i}^{I}(t) d t+\sum_{i=1}^{p} \int_{0}^{1}\left(\sum_{\left\{j: j \in P_{i} \cap N\right\}} b_{i j}^{U}(t) \cdot x_{j}^{U}(t)\right) \cdot y_{i}^{I I}(t) d t \\
& -\sum_{j \in N} \int_{0}^{1} y_{j}^{I I I}(t) \cdot x_{j}^{U}(t) d t+\sum_{j \in N} \int_{0}^{1-\delta} y_{j}^{V}(t) \cdot\left[x_{j}^{U}(t+\delta)-x_{j}^{U}(t)\right] d t,
\end{aligned}
$$

and

$$
\begin{aligned}
& \sum_{j \in P} \int_{0}^{1} a_{j}^{U}(t) \cdot x_{j}^{U}(t) d t=\sum_{j \in P}\left[\int_{0}^{\delta} a_{j}^{U}(t) \cdot x_{j}^{U}(t) d t+\int_{\delta}^{1-\delta} a_{j}^{U}(t) \cdot x_{j}^{U}(t) d t+\int_{1-\delta}^{1} a_{j}^{U}(t) \cdot x_{j}^{U}(t) d t\right] \\
& \leq \sum_{j \in P} \int_{0}^{\delta}\left[\sum_{\left\{i: j \in N_{i}\right\}} b_{i j}^{L}(t) \cdot y_{i}^{I}(t)+\sum_{\left\{i: j \in P_{i}\right\}} b_{i j}^{U}(t) \cdot y_{i}^{I I}(t)-y_{j}^{I I I}(t)-y_{j}^{V}(t)\right] \cdot x_{j}^{U}(t) d t \\
& \quad+\sum_{j \in P} \int_{\delta}^{1-\delta}\left[\sum_{\left\{i: j \in N_{i}\right\}} b_{i j}^{L}(t) \cdot y_{i}^{I}(t)\right. \\
& \left.\quad+\sum_{\left\{i: j \in P_{i}\right\}} b_{i j}^{U}(t) \cdot y_{i}^{I I}(t)-y_{j}^{I I I}(t)-y_{j}^{V}(t)+y_{j}^{V}(t-\delta)\right] \cdot x_{j}^{U}(t) d t \\
& \quad+\sum_{j \in P} \int_{1-\delta}^{1}\left[\sum_{\left\{i: j \in N_{i}\right\}} b_{i j}^{L}(t) \cdot y_{i}^{I}(t)+\sum_{\left\{i: j \in P_{i}\right\}} b_{i j}^{U}(t) \cdot y_{i}^{I I}(t)-y_{j}^{I I I}(t)+y_{j}^{V}(t-\delta)\right] \cdot x_{j}^{U}(t) d t \\
& \left.\quad \text { (using the constraints }(22),(26) \text { and }(30) \text { of dual problem }\left(\mathrm{DSOP}_{\delta}\right)\right)
\end{aligned}
$$

$$
\begin{aligned}
= & \sum_{i=1}^{p} \int_{0}^{1}\left(\sum_{\left\{j: j \in N_{i} \cap P\right\}} b_{i j}^{L}(t) \cdot x_{j}^{U}(t)\right) \cdot y_{i}^{I}(t) d t+\sum_{i=1}^{p} \int_{0}^{1}\left(\sum_{\left\{j: j \in P_{i} \cap P\right\}} b_{i j}^{U}(t) \cdot x_{j}^{U}(t)\right) \cdot y_{i}^{I I}(t) d t \\
& -\sum_{j \in P} \int_{0}^{1} y_{j}^{I I I}(t) \cdot x_{j}^{U}(t) d t-\sum_{j \in P} \int_{0}^{1-\delta} y_{j}^{V}(t) \cdot x_{j}^{U}(t) d t+\sum_{j \in P} \int_{\delta}^{1} y_{j}^{V}(t-\delta) \cdot x_{j}^{U}(t) d t \\
= & \sum_{i=1}^{p} \int_{0}^{1}\left(\sum_{\left\{j: j \in N_{i} \cap P\right\}} b_{i j}^{L}(t) \cdot x_{j}^{U}(t)\right) \cdot y_{i}^{I}(t) d t+\sum_{i=1}^{p} \int_{0}^{1}\left(\sum_{\left\{j: j \in P_{i} \cap P\right\}} b_{i j}^{U}(t) \cdot x_{j}^{U}(t)\right) \cdot y_{i}^{I I}(t) d t \\
& -\sum_{j \in P} \int_{0}^{1} y_{j}^{I I I}(t) \cdot x_{j}^{U}(t) d t+\sum_{j \in P} \int_{0}^{1-\delta} y_{j}^{V}(t) \cdot\left[x_{j}^{U}(t+\delta)-x_{j}^{U}(t)\right] d t,
\end{aligned}
$$

and

$$
\sum_{j \in N} \int_{0}^{1} a_{j}^{U}(t) \cdot x_{j}^{L}(t) d t=\sum_{j \in N}\left[\int_{0}^{\delta} a_{j}^{U}(t) \cdot x_{j}^{L}(t) d t+\int_{\delta}^{1-\delta} a_{j}^{U}(t) \cdot x_{j}^{L}(t) d t+\int_{1-\delta}^{1} a_{j}^{U}(t) \cdot x_{j}^{L}(t) d t\right]
$$




$$
\begin{aligned}
& \leq \sum_{j \in N} \int_{0}^{\delta}\left[\sum_{\left\{i: j \in P_{i}\right\}} b_{i j}^{L}(t) \cdot y_{i}^{I}(t)+\sum_{\left\{i: j \in N_{i}\right\}} b_{i j}^{U}(t) \cdot y_{i}^{I I}(t)+y_{j}^{I I I}(t)+y_{j}^{I V}(t)\right] \cdot x_{j}^{L}(t) d t \\
&+ \sum_{j \in N} \int_{\delta}^{1-\delta}\left[\sum_{\left\{i: j \in P_{i}\right\}} b_{i j}^{L}(t) \cdot y_{i}^{I}(t)\right. \\
&\left.+\sum_{\left\{i: j \in N_{i}\right\}} b_{i j}^{U}(t) \cdot y_{i}^{I I}(t)+y_{j}^{I I I}(t)+y_{j}^{I V}(t)-y_{j}^{I V}(t-\delta)\right] \cdot x_{j}^{L}(t) d t \\
&+\sum_{j \in N} \int_{1-\delta}^{1}\left[\sum_{\left\{i: j \in P_{i}\right\}} b_{i j}^{L}(t) \cdot y_{i}^{I}(t)+\sum_{\left\{i: j \in N_{i}\right\}} b_{i j}^{U}(t) \cdot y_{i}^{I I}(t)+y_{j}^{I I I}(t)-y_{j}^{I V}(t-\delta)\right] \cdot x_{j}^{L}(t) d t
\end{aligned}
$$

(using the constraints (20), (24) and (28) of dual problem (DSOP $\delta$ ))

$$
\begin{aligned}
= & \sum_{i=1}^{p} \int_{0}^{1}\left(\sum_{\left\{j: j \in P_{i} \cap N\right\}} b_{i j}^{L}(t) \cdot x_{j}^{L}(t)\right) \cdot y_{i}^{I}(t) d t+\sum_{i=1}^{p} \int_{0}^{1}\left(\sum_{\left\{j: j \in N_{i} \cap N\right\}} b_{i j}^{U}(t) \cdot x_{j}^{L}(t)\right) \cdot y_{i}^{I I}(t) d t \\
& +\sum_{j \in N} \int_{0}^{1} y_{j}^{I I I}(t) \cdot x_{j}^{L}(t) d t+\sum_{j \in N} \int_{0}^{1-\delta} y_{j}^{I V}(t) \cdot x_{j}^{L}(t) d t-\sum_{j \in N} \int_{\delta}^{1} y_{j}^{I V}(t-\delta) \cdot x_{j}^{L}(t) d t \\
= & \sum_{i=1}^{p} \int_{0}^{1}\left(\sum_{\left\{j: j \in P_{i} \cap N\right\}} b_{i j}^{L}(t) \cdot x_{j}^{L}(t)\right) \cdot y_{i}^{I}(t) d t+\sum_{i=1}^{p} \int_{0}^{1}\left(\sum_{\left\{j: j \in N_{i} \cap N\right\}} b_{i j}^{U}(t) \cdot x_{j}^{L}(t)\right) \cdot y_{i}^{I I}(t) d t \\
& +\sum_{j \in N} \int_{0}^{1} y_{j}^{I I I}(t) \cdot x_{j}^{L}(t) d t+\sum_{j \in N} \int_{0}^{1-\delta} y_{j}^{I V}(t) \cdot\left[x_{j}^{L}(t)-x_{j}^{L}(t+\delta)\right] d t .
\end{aligned}
$$

Therefore, using (32)-(35), we obtain

$$
\begin{aligned}
\sum_{j \in P} \int_{0}^{1} a_{j}^{L}(t) \cdot x_{j}^{L}(t) d \alpha+\sum_{j \in N} \int_{0}^{1} a_{j}^{U}(t) \cdot x_{j}^{L}(t) d \alpha+\sum_{j \in N} \int_{0}^{1} a_{j}^{L}(t) \cdot x_{j}^{U}(t) d \alpha+\sum_{j \in P} \int_{0}^{1} a_{j}^{U}(t) \cdot x_{j}^{U}(t) d \alpha \\
\leq \sum_{i=1}^{p} \int_{0}^{1}\left(\sum_{\left\{j: j \in P_{i}\right\}} b_{i j}^{L}(t) \cdot x_{j}^{L}(t)+\sum_{\left\{j: j \in N_{i}\right\}} b_{i j}^{L}(t) \cdot x_{j}^{U}(t)\right) \cdot y_{i}^{I}(t) d t \\
\quad+\sum_{i=1}^{p} \int_{0}^{1}\left(\sum_{\left\{j: j \in N_{i}\right\}} b_{i j}^{U}(t) \cdot x_{j}^{L}(t)+\sum_{\left\{j: j \in P_{i}\right\}} b_{i j}^{U}(t) \cdot x_{j}^{U}(t)\right) \cdot y_{i}^{I I}(t) d t \\
\quad+\sum_{j=1}^{q} \int_{0}^{1} y_{j}^{I I I}(t) \cdot\left[x_{j}^{L}(t)-x_{j}^{U}(t)\right] d t \\
\quad+\sum_{j=1}^{q} \int_{0}^{1-\delta} y_{j}^{I V}(t) \cdot\left[x_{j}^{L}(t)-x_{j}^{L}(t+\delta)\right]+\sum_{j=1}^{q} \int_{0}^{1-\delta} y_{j}^{V}(t) \cdot\left[x_{j}^{U}(t+\delta)-x_{j}^{U}(t)\right] d t \\
\quad \leq \sum_{i=1}^{p} \int_{0}^{1} c_{i}^{L}(t) \cdot y_{i}^{I}(t) d t+\sum_{i=1}^{p} \int_{0}^{1} c_{i}^{U}(t) \cdot y_{i}^{I I}(t) d t\left(\text { by the constraint of }\left(\operatorname{SOP}_{\delta}\right)\right),
\end{aligned}
$$

which also says that $V\left(\mathrm{SOP}_{\delta}\right) \leq V\left(\mathrm{DSOP}_{\delta}\right)$. From Remark 2, we see that $V(\mathrm{SOP}) \leq V\left(\mathrm{SOP}_{\delta}\right)$. This completes the proof. 
For convenient discussion, we adopt the following notations:

$$
\tau^{L}=\max _{j=1, \cdots, q} \sup _{t \in[0,1]} a_{j}^{L}(t) \text { and } \tau^{U}=\max _{j=1, \cdots, q} \sup _{t \in[0,1]} a_{j}^{U}(t),
$$

and

$$
\mu^{L}=\max _{i=1, \cdots, p} \sup _{t \in[0,1]} c_{i}^{L}(t) \text { and } \mu^{U}=\max _{i=1, \cdots, p} \sup _{t \in[0,1]} c_{i}^{U}(t) .
$$

It is clear to see that the primal problem $\left(\mathrm{SOP}_{\delta}\right)$ is feasible, since the zero functions form a feasible solution. The following result presents the feasibility of dual problem $\left(\mathrm{DSOP}_{\delta}\right)$.

Proposition 3. For $i=1, \cdots, p$, we define the constant functions on $[0,1]$ as follows

$$
y_{i}^{I}(t)=y_{i}^{I I}(t)=\max \left\{\frac{\tau^{L}}{\sigma^{P N}}, \frac{\tau^{U}}{\sigma^{P N}}, \frac{\tau^{L}}{\sigma^{N P}} \frac{\tau^{U}}{\sigma^{N P}}\right\} \text { for } t \in[0,1] .
$$

For $j=1, \cdots, q$, we define

$$
y_{j}^{I I I}(t)=y_{j}^{I V}(t)=y_{j}^{V}(t)=0 \text { for } t \in[0,1] .
$$

Then $\mathbf{y}=\left(\mathbf{y}^{I}, \mathbf{y}^{I I}, \mathbf{y}^{I I I}, \mathbf{y}^{I V}, \mathbf{y}^{V}\right)$ is a feasible solution of problem $\left(\mathrm{DSOP}_{\delta}\right)$. Moreover we have

$$
0 \leq V\left(\operatorname{DSOP}_{\delta}\right) \leq p \cdot \max \left\{\frac{\tau^{L}}{\sigma^{P N}}, \frac{\tau^{U}}{\sigma^{P N}}, \frac{\tau^{L}}{\sigma^{N P}} \frac{\tau^{U}}{\sigma^{N P}}\right\} \cdot\left(\mu^{L}+\mu^{U}\right)
$$

for any $\delta$ with $0<\delta<1$.

Proof. It is clear to see that $\mathbf{y}$ is nonnegative. Let

$$
\mathfrak{y}=\max \left\{\frac{\tau^{L}}{\sigma^{P N}}, \frac{\tau^{U}}{\sigma^{P N}}, \frac{\tau^{L}}{\sigma^{N P}} \frac{\tau^{U}}{\sigma^{N P}}\right\} \geq 0 .
$$

By substituting $\mathbf{y}$ into the constraints of $\left(\operatorname{DSOP}_{\delta}\right)$, we have, for $l=1, \cdots, n$ and $j=1, \cdots, q$,

$$
\begin{aligned}
& \sum_{\left\{i: j \in P_{i}\right\}} b_{i j}^{L}(t) \cdot y_{i}^{I}(t)+\sum_{\left\{i: j \in N_{i}\right\}} b_{i j}^{U}(t) \cdot y_{i}^{I I}(t)=\mathfrak{y} \cdot\left(\sum_{\left\{i: j \in P_{i}\right\}} b_{i j}^{L}(t)+\sum_{\left\{i: j \in N_{i}\right\}} b_{i j}^{U}(t)\right) \\
& \geq \mathfrak{y} \cdot \sigma^{P N} \text { (using (15)) } \\
& \geq \frac{\max \left\{\tau^{L}, \tau^{U}\right\}}{\sigma^{P N}} \cdot \sigma^{P N}=\max \left\{\tau^{L}, \tau^{U}\right\} \geq\left\{\begin{array}{l}
\tau^{L} \geq a_{j}^{L}(t) \quad \text { if } j \in P \\
\tau^{U} \geq a_{j}^{U}(t) \text { if } j \in N
\end{array}\right. \text { (using (36)), }
\end{aligned}
$$

and

$$
\begin{aligned}
& \sum_{\left\{i: j \in N_{i}\right\}} b_{i j}^{L}(t) \cdot y_{i}^{I}(t)+\sum_{\left\{i: j \in P_{i}\right\}} b_{i j}^{U}(t) \cdot y_{i}^{I I}(t)=\mathfrak{y} \cdot\left(\sum_{\left\{i: j \in N_{i}\right\}} b_{i j}^{L}(t)+\sum_{\left\{i: j \in P_{i}\right\}} b_{i j}^{U}(t)\right) \\
& \geq \mathfrak{y} \cdot \sigma^{N P} \text { (using (16)) }
\end{aligned}
$$

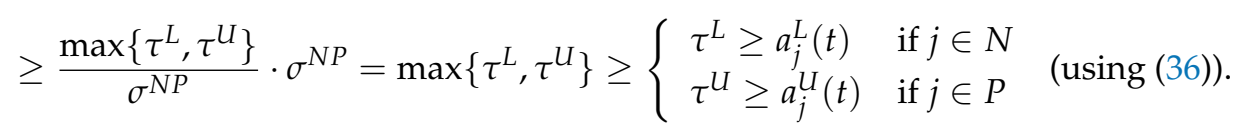


This shows that $\mathbf{y}$ is a feasible solution of problem $\left(\mathrm{DSOP}_{\delta}\right)$ with objective value

$$
\begin{aligned}
& \sum_{i=1}^{p} \int_{0}^{1} c_{i}^{L}(t) \cdot y_{i}^{I}(t) d t+\sum_{i=1}^{p} \int_{0}^{1} c_{i}^{U}(t) \cdot y_{i}^{I I}(t) d t \\
& \quad \leq \sum_{i=1}^{p} \int_{0}^{1} \mu^{L} \cdot y_{i}^{I}(t) d t+\sum_{i=1}^{p} \int_{0}^{1} \mu^{U} \cdot y_{i}^{I I}(t) d t=p \cdot \mathfrak{y} \cdot\left(\mu^{L}+\mu^{U}\right) .
\end{aligned}
$$

The inequality (38) is obvious, since the dual problem $\left(\mathrm{DSOP}_{\delta}\right)$ is a minimization problem. This completes the proof.

\section{Discretization}

In order to solve problem SOP, we need to consider the discretization of problem SOP by equally dividing the unit interval $[0,1]$ into $n$ subintervals. In this case, the discretized problem will turn into a conventional linear programming problem.

We assume that the closed interval $[0,1]$ is equally divided into $n$ closed subintervals such that the length of subinterval is $1 / n$. Let $\mathcal{P}_{n}=\left\{e_{0}^{(n)}, e_{1}^{(n)}, \ldots, e_{n}^{(n)}\right\}$ be a partition of $[0,1]$, where $e_{0}^{(n)}=0$ and $\frac{l}{n}=l / n$ for $l=1, \cdots, n$. The $n$ closed subintervals are denoted by

$$
\bar{E}_{l}^{(n)}=\left[\frac{l-1}{n}, \frac{l}{n}\right] \text { for } l=1, \cdots, n .
$$

We also write

$$
E_{l}^{(n)}=\left(\frac{l-1}{n}, \frac{l}{n}\right) \text { and } F_{l}^{(n)}=\left[\frac{l-1}{n}, \frac{l}{n}\right)
$$

We assume that the real-valued functions $a_{j}^{L}, a_{j}^{U}, c_{i}^{L}, c_{i}^{U}, b_{i j}^{L}$ and $b_{i j}^{U}$ are all continuous on $[0,1]$ for $i=1, \cdots, p$ and $j=1, \cdots, q$. Now we define

$$
\begin{aligned}
& a_{l j}^{(L, n)}=\inf _{t \in \bar{E}_{l}^{(n)}} a_{j}^{L}(t) \text { and } a_{l j}^{(U, n)}=\inf _{t \in \bar{E}_{l}^{(n)}} a_{j}^{U}(t) \\
& c_{l i}^{(L, n)}=\inf _{t \in \bar{E}_{l}^{(n)}} c_{i}^{L}(t) \text { and } c_{l i}^{(U, n)}=\inf _{t \in \bar{E}_{l}^{(n)}} c_{i}^{U}(t) \\
& b_{l i j}^{(L, n)}=\sup _{t \in \bar{E}_{l}^{(n)}} b_{i j}^{L}(t) \text { and } b_{l i j}^{(U, n)}=\sup _{t \in \bar{E}_{l}^{(n)}} b_{i j}^{U}(t) .
\end{aligned}
$$

Since the functions $a_{j}^{L}, c_{i}^{L}$ and $b_{i j}^{L}$ are increasing on $[0,1]$ and the functions $a_{j}^{U}, c_{i}^{U}$ and $b_{i j}^{U}$ are decreasing on $[0,1]$ for $i=1, \cdots, p$ and $j=1, \cdots, q$, we have

$$
\begin{aligned}
& a_{l j}^{(L, n)}=a_{j}^{L}\left(\frac{l-1}{n}\right) \text { and } a_{l j}^{(U, n)}=a_{j}^{U}\left(\frac{l}{n}\right) \\
& c_{l i}^{(L, n)}=c_{i}^{L}\left(\frac{l-1}{n}\right) \text { and } c_{l i}^{(U, n)}=c_{i}^{U}\left(\frac{l}{n}\right) \\
& b_{l i j}^{(L, n)}=b_{i j}^{L}\left(\frac{l}{n}\right) \text { and } b_{l i j}^{(U, n)}=b_{i j}^{U}\left(\frac{l-1}{n}\right) .
\end{aligned}
$$


Now we are in a position to discretize the problem SOP as follows. For each $n \in \mathbb{N}$ and $l=$ $1, \cdots, n$, we define the following linear programming problem:

$\left(\mathrm{P}_{n}\right) \quad \max$

$$
\begin{aligned}
\sum_{j \in P} & \left(\sum_{l=1}^{n} \frac{1}{n} \cdot a_{l j}^{(L, n)} \cdot z_{l j}^{L}+\sum_{l=1}^{n} \frac{1}{n} \cdot a_{l j}^{(U, n)} \cdot z_{l j}^{U}\right) \\
& +\sum_{j \in N}\left(\sum_{l=1}^{n} \frac{1}{n} \cdot a_{l j}^{(U, n)} \cdot z_{l j}^{L}+\sum_{l=1}^{n} \frac{1}{n} \cdot a_{l j}^{(L, n)} \cdot z_{l j}^{U}\right)
\end{aligned}
$$

subject to $\sum_{\left\{j: j \in P_{i}\right\}} b_{l i j}^{(L, n)} z_{l j}^{L}+\sum_{\left\{j: j \in N_{i}\right\}} b_{l i j}^{(L, n)} z_{l j}^{U} \leq c_{l i}^{(L, n)}$ for $i=1, \cdots, p$ and $l=1, \cdots, n$;

$$
\begin{aligned}
& \sum_{\left\{j: j \in N_{i}\right\}} b_{l i j}^{(U, n)} z_{l j}^{L}+\sum_{\left\{j: j \in P_{i}\right\}} b_{l i j}^{(U, n)} z_{l j}^{U} \leq c_{l i}^{(U, n)} \text { for } i=1, \cdots, p \text { and } l=1, \cdots, n ; \\
& z_{l j}^{L} \leq z_{l j}^{U} \text { for } j=1, \cdots, q \text { and } l=1, \cdots, n ; \\
& z_{l j}^{L} \leq z_{l+1, j}^{L} \text { for } j=1, \cdots, q \text { and } l=1, \cdots, n-1 ; \\
& z_{l+1, j}^{U} \leq z_{l j}^{U} \text { for } j=1, \cdots, q \text { and } l=1, \cdots, n-1 ; \\
& z_{l j}^{L} \geq 0 \text { and } z_{l j}^{U} \geq 0 \text { for } j=1, \cdots, q \text { and } l=1, \cdots, n,
\end{aligned}
$$

where $z_{l j}^{L}$ and $z_{l j}^{U}$ are nonnegative decision variables for $j=1, \cdots, q$ and $l=1, \cdots, n$. We write

$$
\mathbf{z}_{l}=\left(\mathbf{z}_{l}^{L}, \mathbf{z}_{l}^{U}\right)=\left(z_{l 1}^{L}, \cdots, z_{l q}^{L}, z_{l 1}^{U}, \cdots, z_{l q}^{U}\right) \text { and } \mathbf{z}=\left(\mathbf{z}_{1}, \cdots, \mathbf{z}_{n}\right)
$$

to denote the feasible solution of problem $\left(\mathrm{P}_{n}\right)$.

In order to formulate the dual problem of $\left(\mathrm{P}_{n}\right)$, we need to write it as a compact form in terms of matrices. For $l=1, \cdots, n$, we consider the following vector

$$
\mathbf{c}_{l}^{(n)}=\left(\mathbf{c}_{l}^{(L, n)}, \mathbf{c}_{l}^{(U, n)}\right)=\left(c_{l 1}^{(L, n)}, \ldots, c_{l p}^{(L, n)}, c_{l 1}^{(U, n)}, \ldots, c_{l p}^{(U, n)}\right) \text { and } \mathbf{c}^{(n)}=\left(\mathbf{c}_{1}^{(n)}, \ldots, \mathbf{c}_{n}^{(n)}\right) .
$$

For $l=1, \cdots, n$, we define the following vectors:

- $\quad \mathbf{a}_{l}^{(P N, n)}$ is a vector for $l=1, \cdots, n$ such that its $j$-th entry is $a_{l j}^{(L, n)}$ for $j \in P$ and $a_{l j}^{(U, n)}$ for $j \in N$.

- $\quad \mathbf{a}_{l}^{(N P, n)}$ is a vector for $l=1, \cdots, n$ such that its $j$-th entry is $a_{l j}^{(L, n)}$ for $j \in N$ and $a_{l j}^{(U, n)}$ for $j \in P$.

We also write

$$
\mathbf{a}_{l}^{(n)}=\left(\mathbf{a}_{l}^{(P N, n)}, \mathbf{a}_{l}^{(N P, n)}\right) \text { and } \mathbf{a}^{(n)}=\left(\mathbf{a}_{1}^{(n)}, \cdots, \mathbf{a}_{n}^{(n)}\right) .
$$

We define the following matrices:

- $\quad B_{l}^{(P L, n)}$ is a $p \times q$ matrix for $l=1, \cdots, n$ such that its $i j$-th entry is $b_{l i j}^{(L, n)}$ for $j \in P_{i} \neq \varnothing$ and 0 for $j \in N_{i}$. If $P_{i}=\varnothing$, then $b_{l i j}^{(L, n)}=0$ for $j=1, \cdots, q$.

- $\quad B_{l}^{(N L, n)}$ is a $p \times q$ matrix for $l=1, \cdots, n$ such that its $i j$-th entry is $b_{l i j}^{(L, n)}$ for $j \in N_{i} \neq \varnothing$ and 0 for $j \in P_{i}$. If $N_{i}=\varnothing$, then $b_{l i j}^{(L, n)}=0$ for $j=1, \cdots, q$.

- $\quad B_{l}^{(P U, n)}$ is a $p \times q$ matrix for $l=1, \cdots, n$ such that its $i j$-th entry is $b_{l i j}^{(U, n)}$ for $j \in P_{i} \neq \varnothing$ and 0 for $j \in N_{i}$. If $P_{i}=\varnothing$, then $b_{l i j}^{(L, n)}=0$ for $j=1, \cdots, q$.

- $\quad B_{l}^{(N U, n)}$ is a $p \times q$ matrix for $l=1, \cdots, n$ such that its $i j$-th entry is $b_{l i j}^{(U, n)}$ for $j \in N_{i} \neq \varnothing$ and 0 for $j \in P_{i}$. If $N_{i}=\varnothing$, then $b_{l i j}^{(L, n)}=0$ for $j=1, \cdots, q$. 
We also define the following $2 p \times 2 q$ matrices

$$
B_{l}^{(n)}=\left[\begin{array}{cc}
B_{l}^{(P L, n)} & B_{l}^{(N L, n)} \\
B_{l}^{(N U, n)} & B_{l}^{(P U, n)}
\end{array}\right]
$$

for $l=1, \cdots, n$. Let $I_{q \times q}$ denote a $q \times q$ identity matrix, i.e., the entries in the diagonal are all 1 's and the remaining entries are all 0 's. We also define a $q \times 2 q$ matrix $A$ and a $2 q \times 2 q$ matrix $C$ by

$$
A=\left[I_{q \times q},-I_{q \times q}\right] \text { and } C=\left[\begin{array}{cc}
I_{q \times q} & \mathbf{0}_{q \times q} \\
\mathbf{0}_{q \times q} & -I_{q \times q}
\end{array}\right]
$$

Then the linear programming problem $\left(\mathrm{P}_{n}\right)$ can be rewritten as follows:

$$
\begin{aligned}
\left(\mathrm{P}_{n}\right) \quad \max \quad & \sum_{l=1}^{n} \frac{1}{n} \cdot\left(\mathbf{a}_{l}^{(n)}\right)^{\top} \mathbf{z}_{l}=\sum_{l=1}^{n} \frac{1}{n} \cdot\left(\mathbf{a}_{l}^{(P N, n)}\right)^{\top} \mathbf{z}_{l}^{L}+\sum_{l=1}^{n} \frac{1}{n} \cdot\left(\mathbf{a}_{l}^{(N P, n)}\right)^{\top} \mathbf{z}_{l}^{U} \\
\text { subject to } \quad & B_{l}^{(n)} \mathbf{z}_{l} \leq \mathbf{c}_{l}^{(n)} \text { for } l=1, \cdots, n ; \\
& A \mathbf{z}_{l} \leq \mathbf{0}_{1 \times q} \text { for } l=1, \cdots, n ; \\
& C \mathbf{z}_{l}-C \mathbf{z}_{l+1} \leq \mathbf{0}_{1 \times 2 q} \text { for } l=1, \cdots, n-1 ; \\
& \mathbf{z}_{l} \in \mathbb{R}_{+}^{2 q} \text { for } l=1, \cdots, n .
\end{aligned}
$$

We also write it as the following standard form:

$$
\begin{array}{lll}
\left(\mathrm{P}_{n}\right) & \max & \mathbf{b}^{\top} \mathbf{z} \\
\text { subject to } & M \mathbf{z} \leq \overline{\mathbf{b}} \text { and } \mathbf{z} \geq \mathbf{0},
\end{array}
$$

where

$$
\begin{gathered}
\mathbf{z}=\left(\mathbf{z}_{1}, \cdots, \mathbf{z}_{l}, \cdots, \mathbf{z}_{n}\right)^{\top} \\
\overline{\mathbf{b}}=\left(\mathbf{c}_{1}^{(n)}, \cdots, \mathbf{c}_{l}^{(n)}, \cdots, \mathbf{c}_{n}^{(n)}, \mathbf{0}_{1 \times n q}, \mathbf{0}_{1 \times 2(n-1) q}\right)^{\top} \\
\mathbf{b}=\left(\frac{\mathbf{a}_{1}^{(n)}}{n}, \cdots, \frac{\mathbf{a}_{l}^{(n)}}{n}, \cdots, \frac{\mathbf{a}_{n}^{(n)}}{n}\right)^{\top},
\end{gathered}
$$


and

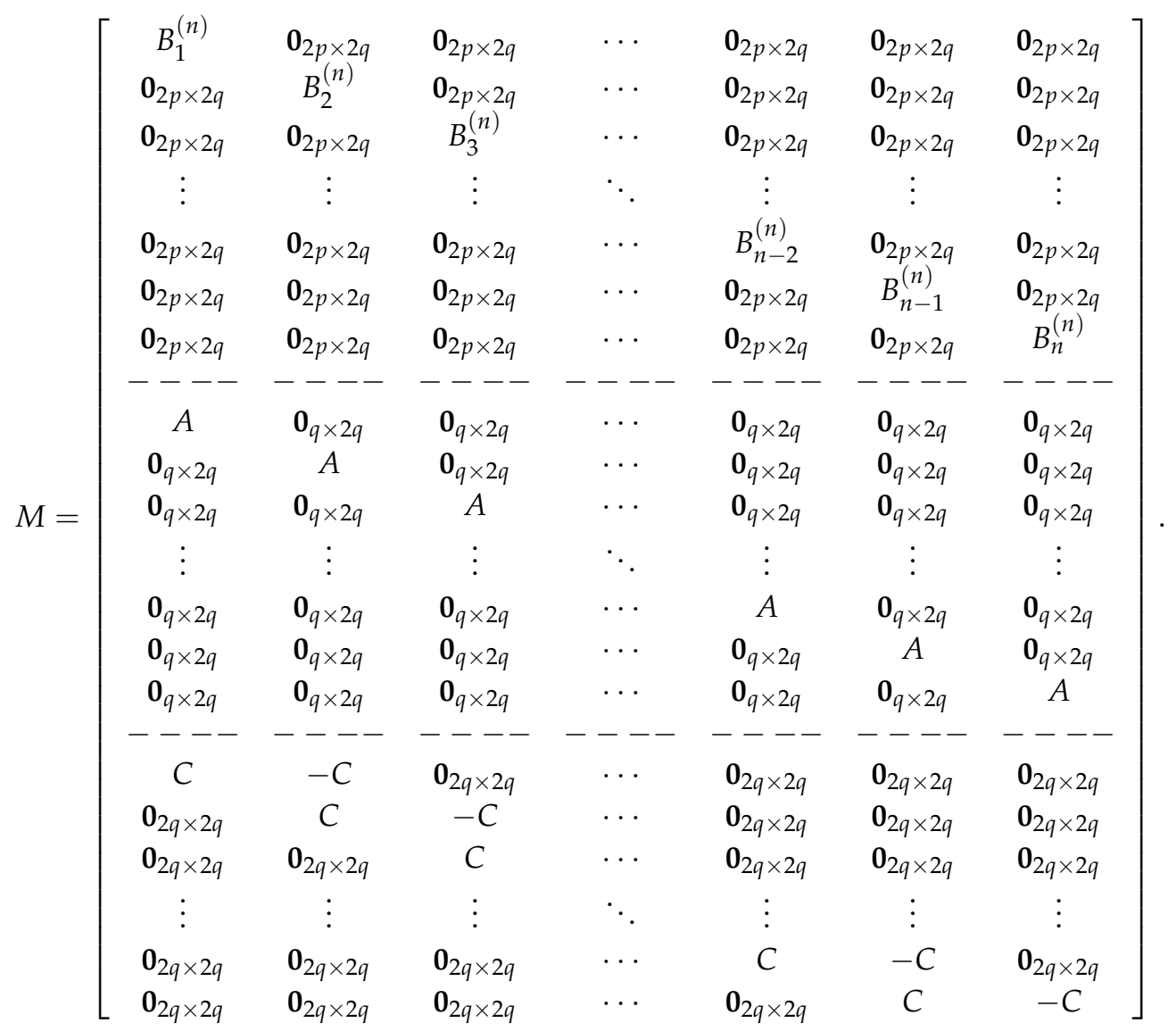

Then the dual problem of $\left(\mathrm{P}_{n}\right)$ is given by

$$
\begin{array}{ccl}
\left(\widehat{\mathrm{D}}_{n}\right) \quad \min & \overline{\mathbf{b}}^{\top} \widehat{\mathbf{w}} \\
& \text { subject to } & M^{\top} \widehat{\mathbf{w}} \geq \mathbf{b} \text { and } \widehat{\mathbf{w}} \geq \mathbf{0},
\end{array}
$$

where

$$
\widehat{\mathbf{w}}=\left(\widehat{\mathbf{w}}^{B}, \widehat{\mathbf{w}}^{A}, \widehat{\mathbf{w}}^{C}\right)^{\top}=\left(\widehat{\mathbf{w}}^{I}, \widehat{\mathbf{w}}^{I I}, \widehat{\mathbf{w}}^{I I}, \widehat{\mathbf{w}}^{I V}, \widehat{\mathbf{w}}^{V}\right)^{\top}
$$

with

$$
\widehat{\mathbf{w}}^{B}=\left(\widehat{\mathbf{w}}^{I}, \widehat{\mathbf{w}}^{I I}\right), \quad \widehat{\mathbf{w}}^{A}=\widehat{\mathbf{w}}^{I I I}, \quad \widehat{\mathbf{w}}^{C}=\left(\widehat{\mathbf{w}}^{I V}, \widehat{\mathbf{w}}^{V}\right)
$$

and

$$
M^{\top}=[\mathcal{B}|\mathcal{A}| \mathcal{C}]
$$

with

$$
\mathcal{B}=\left[\begin{array}{cccccc}
\left(B_{1}^{(n)}\right)^{\top} & \mathbf{0}_{2 q \times 2 p} & \mathbf{0}_{2 q \times 2 p} & \cdots & \mathbf{0}_{2 q \times 2 p} & \mathbf{0}_{2 q \times 2 p} \\
\mathbf{0}_{2 q \times 2 p} & \left(B_{2}^{(n)}\right)^{\top} & \mathbf{0}_{2 q \times 2 p} & \cdots & \mathbf{0}_{2 q \times 2 p} & \mathbf{0}_{2 q \times 2 p} \\
\mathbf{0}_{2 q \times 2 p} & \mathbf{0}_{2 q \times 2 p} & \left(B_{3}^{(n)}\right)^{\top} & \cdots & \mathbf{0}_{2 q \times 2 p} & \mathbf{0}_{2 q \times 2 p} \\
\vdots & \vdots & \vdots & \ddots & \vdots & \vdots \\
\mathbf{0}_{2 q \times 2 p} & \mathbf{0}_{2 q \times 2 p} & \mathbf{0}_{2 q \times 2 p} & \cdots & \left(B_{n-1}^{(n)}\right)^{\top} & \mathbf{0}_{2 q \times 2 p} \\
\mathbf{0}_{2 q \times 2 p} & \mathbf{0}_{2 q \times 2 p} & \mathbf{0}_{2 q \times 2 p} & \cdots & \mathbf{0}_{2 q \times 2 p} & \left(B_{n}^{(n)}\right)^{\top}
\end{array}\right]
$$




$$
\begin{gathered}
\mathcal{A}=\left[\begin{array}{cccccc}
A^{\top} & \mathbf{0}_{2 q \times q} & \mathbf{0}_{2 q \times q} & \cdots & \mathbf{0}_{2 q \times q} & \mathbf{0}_{2 q \times q} \\
\mathbf{0}_{2 q \times q} & A^{\top} & \mathbf{0}_{2 q \times q} & \cdots & \mathbf{0}_{2 q \times q} & \mathbf{0}_{2 q \times q} \\
\mathbf{0}_{2 q \times q} & \mathbf{0}_{2 q \times q} & A^{\top} & \cdots & \mathbf{0}_{2 q \times q} & \mathbf{0}_{2 q \times q} \\
\vdots & \vdots & \vdots & \ddots & \vdots & \vdots \\
\mathbf{0}_{2 q \times q} & \mathbf{0}_{2 q \times q} & \mathbf{0}_{2 q \times q} & \cdots & A^{\top} & \mathbf{0}_{2 q \times q} \\
\mathbf{0}_{2 q \times q} & \mathbf{0}_{2 q \times q} & \mathbf{0}_{2 q \times q} & \cdots & \mathbf{0}_{2 q \times q} & A^{\top}
\end{array}\right] \\
\mathcal{C}=\left[\begin{array}{ccccccc}
C^{\top} & \mathbf{0}_{2 q \times 2 q} & \mathbf{0}_{2 q \times 2 q} & \cdots & \mathbf{0}_{2 q \times 2 q} & \mathbf{0}_{2 q \times 2 q} & \mathbf{0}_{2 q \times 2 q} \\
-C^{\top} & C^{\top} & \mathbf{0}_{2 q \times 2 q} & \cdots & \mathbf{0}_{2 q \times 2 q} & \mathbf{0}_{2 q \times 2 q} & \mathbf{0}_{2 q \times 2 q} \\
\mathbf{0}_{2 q \times 2 q} & -C^{\top} & C^{\top} & \cdots & \mathbf{0}_{2 q \times 2 q} & \mathbf{0}_{2 q \times 2 q} & \mathbf{0}_{2 q \times 2 q} \\
\vdots & \vdots & \vdots & \vdots & \vdots & \vdots & \vdots \\
\mathbf{0}_{2 q \times 2 q} & \mathbf{0}_{2 q \times 2 q} & \mathbf{0}_{2 q \times 2} & \cdots & -C^{\top} & C^{\top} & \mathbf{0}_{2 q \times 2 q} \\
\mathbf{0}_{2 q \times 2 q} & \mathbf{0}_{2 q \times 2 q} & \mathbf{0}_{2 q \times 2} q & \cdots & \mathbf{0}_{2 q \times 2 q} & -C^{\top} & C^{\top} \\
\mathbf{0}_{2 q \times 2 q} & \mathbf{0}_{2 q \times 2 q} & \mathbf{0}_{2 q \times 2} & \cdots & \mathbf{0}_{2 q \times 2 q} & \mathbf{0}_{2 q \times 2 q} & -C^{\top}
\end{array}\right] .
\end{gathered}
$$

The dual problem $\left(\widehat{\mathrm{D}}_{n}\right)$ is written by

$$
\begin{aligned}
\left(\widehat{\mathbf{D}}_{n}\right) \quad \min \quad & \sum_{l=1}^{n}\left(\mathbf{c}_{l}^{(n)}\right)^{\top} \widehat{\mathbf{w}}_{l}^{B} \\
\text { subject to } & \left(B_{1}^{(n)}\right)^{\top} \widehat{\mathbf{w}}_{1}^{B}+A^{\top} \widehat{\mathbf{w}}_{1}^{A}+C^{\top} \widehat{\mathbf{w}}_{1}^{C} \geq \frac{1}{n} \mathbf{a}_{1}^{(n)} ; \\
& \left(B_{l}^{(n)}\right)^{\top} \widehat{\mathbf{w}}_{l}^{B}+A^{\top} \widehat{\mathbf{w}}_{l}^{A}+C^{\top} \widehat{\mathbf{w}}_{l}^{C}-C^{\top} \widehat{\mathbf{w}}_{l-1}^{C} \geq \frac{1}{n} \mathbf{a}_{l}^{(n)} \text { for } l=2, \cdots, n-1 ; \\
& \left(B_{n}^{(n)}\right)^{\top} \widehat{\mathbf{w}}_{n}^{B}+A^{\top} \widehat{\mathbf{w}}_{n}^{A}-C^{\top} \widehat{\mathbf{w}}_{n-1}^{C} \geq \frac{1}{n} \mathbf{a}_{n}^{(n)} ; \\
& \widehat{\mathbf{w}}_{l}^{B} \in \mathbb{R}_{+}^{2 p} \text { and } \widehat{\mathbf{w}}_{l}^{A} \in \mathbb{R}_{+}^{q} \text { for } l=1, \cdots, n ; \\
& \widehat{\mathbf{w}}_{l}^{C} \in \mathbb{R}_{+}^{2 q} \text { for } l=1, \cdots, n-1 .
\end{aligned}
$$

Now, let

$$
\mathbf{w}_{l}^{A}=n \cdot \widehat{\mathbf{w}}_{l}^{A}, \quad \mathbf{w}_{l}^{B}=n \cdot \widehat{\mathbf{w}}_{l}^{B} \text { and } \mathbf{w}_{l}^{C}=n \cdot \widehat{\mathbf{w}}_{l}^{C} .
$$

By multiplying $n$ on both sides of the constraints, the dual problem $\left(\widehat{\mathbf{D}}_{n}\right)$ can be equivalently written by

$$
\begin{aligned}
\left(\mathrm{D}_{n}\right) \quad \min \quad & \sum_{l=1}^{n} \frac{1}{n} \cdot\left(\mathbf{c}_{l}^{(n)}\right)^{\top} \mathbf{w}_{l}^{B} \\
\text { subject to } & \left(B_{1}^{(n)}\right)^{\top} \mathbf{w}_{1}^{B}+A^{\top} \mathbf{w}_{1}^{A}+C^{\top} \mathbf{w}_{1}^{C} \geq \mathbf{a}_{1}^{(n)} ; \\
& \left(B_{l}^{(n)}\right)^{\top} \mathbf{w}_{l}^{B}+A^{\top} \mathbf{w}_{l}^{A}+C^{\top} \mathbf{w}_{l}^{C}-C^{\top} \mathbf{w}_{l-1}^{C} \geq \mathbf{a}_{l}^{(n)} \\
& \text { for } l=2, \cdots, n-1 ; \\
& \left(B_{n}^{(n)}\right)^{\top} \mathbf{w}_{n}^{B}+A^{\top} \mathbf{w}_{n}^{A}-C^{\top} \mathbf{w}_{n-1}^{C} \geq \mathbf{a}_{n}^{(n)} ; \\
& \mathbf{w}_{l}^{B} \in \mathbb{R}_{+}^{2 p} \text { and } \mathbf{w}_{l}^{A} \in \mathbb{R}_{+}^{q} \text { for } l=1, \cdots, n ; \\
& \mathbf{w}_{l}^{C} \in \mathbb{R}_{+}^{2 q} \text { for } l=1, \cdots, n-1 .
\end{aligned}
$$

More precisely, we have the following form

$$
\left(\mathrm{D}_{n}\right) \quad \min \sum_{i=1}^{p} \sum_{l=1}^{n} \frac{1}{n} \cdot c_{l i}^{(L, n)} \cdot w_{l i}^{I}+\sum_{i=1}^{p} \sum_{l=1}^{n} \frac{1}{n} \cdot c_{l i}^{(U, n)} \cdot w_{l i}^{I I}
$$




$$
\begin{aligned}
& \text { subject to } \sum_{\left\{i: j \in P_{i}\right\}} b_{1 i j}^{(L, n)} \cdot w_{1 i}^{I}+\sum_{\left\{i: j \in N_{i}\right\}} b_{1 i j}^{(U, n)} \cdot w_{1 i}^{I I}+w_{1 j}^{I I I}+w_{1 j}^{I V} \geq a_{1 j}^{(L, n)} \text { for } j \in P \text {; } \\
& \sum_{\left\{i: j \in P_{i}\right\}} b_{1 i j}^{(L, n)} \cdot w_{1 i}^{I}+\sum_{\left\{i: j \in N_{i}\right\}} b_{1 i j}^{(U, n)} \cdot w_{1 i}^{I I}+w_{1 j}^{I I I}+w_{1 j}^{I V} \geq a_{1 j}^{(U, n)} \text { for } j \in N \text {; } \\
& \sum_{\left\{i: j \in N_{i}\right\}} b_{1 i j}^{(L, n)} \cdot w_{1 i}^{I}+\sum_{\left\{i: j \in P_{i}\right\}} b_{1 i j}^{(U, n)} \cdot w_{1 i}^{I I}-w_{1 j}^{I I I}-w_{1 j}^{V} \geq a_{1 j}^{(L, n)} \text { for } j \in N \\
& \sum_{\left\{i: j \in N_{i}\right\}} b_{1 i j}^{(L, n)} \cdot w_{1 i}^{I}+\sum_{\left\{i: j \in P_{i}\right\}} b_{1 i j}^{(U, n)} \cdot w_{1 i}^{I I}-w_{1 j}^{I I I}-w_{1 j}^{V} \geq a_{1 j}^{(U, n)} \text { for } j \in P \\
& \sum_{\left\{i: j \in P_{i}\right\}} b_{l i j}^{(L, n)} \cdot w_{l i}^{I}+\sum_{\left\{i: j \in N_{i}\right\}} b_{l i j}^{(U, n)} \cdot w_{l i}^{I I}+w_{l j}^{I I I}+w_{l j}^{I V}-w_{l-1, j}^{I V} \geq a_{l j}^{(L, n)} \\
& \text { for } j \in P \text { and } l=2, \cdots, n-1 \text {; } \\
& \sum_{\left\{i: j \in P_{i}\right\}} b_{l i j}^{(L, n)} \cdot w_{l i}^{I}+\sum_{\left\{i: j \in N_{i}\right\}} b_{l i j}^{(U, n)} \cdot w_{l i}^{I I}+w_{l j}^{I I I}+w_{l j}^{I V}-w_{l-1, j}^{I V} \geq a_{l j}^{(U, n)} \\
& \text { for } j \in N \text { and } l=2, \cdots, n-1 \text {; } \\
& \sum_{\left\{i: j \in N_{i}\right\}} b_{l i j}^{(L, n)} \cdot w_{l i}^{I}+\sum_{\left\{i: j \in P_{i}\right\}} b_{l i j}^{(U, n)} \cdot w_{l i}^{I I}-w_{l j}^{I I I}-w_{l j}^{V}+w_{l-1, j}^{V} \geq a_{l j}^{(L, n)} \\
& \text { for } j \in N \text { and } l=2, \cdots, n-1 \text {; } \\
& \sum_{\left\{i: j \in N_{i}\right\}} b_{l i j}^{(L, n)} \cdot w_{l i}^{I}+\sum_{\left\{i: j \in P_{i}\right\}} b_{l i j}^{(U, n)} \cdot w_{l i}^{I I}-w_{l j}^{I I I}-w_{l j}^{V}+w_{l-1, j}^{V} \geq a_{l j}^{(U, n)} \\
& \text { for } j \in P \text { and } l=2, \cdots, n-1 \text {; } \\
& \sum_{\left\{i: j \in P_{i}\right\}} b_{n i j}^{(L, n)} \cdot w_{n i}^{I}+\sum_{\left\{i: j \in N_{i}\right\}} b_{n i j}^{(U, n)} \cdot w_{n i}^{I I}+w_{n j}^{I I I}-w_{n-1, j}^{I V} \geq a_{n j}^{(L, n)} \text { for } j \in P \\
& \sum_{\left\{i: j \in P_{i}\right\}} b_{n i j}^{(L, n)} \cdot w_{n i}^{I}+\sum_{\left\{i: j \in N_{i}\right\}} b_{n i j}^{(U, n)} \cdot w_{n i}^{I I}+w_{n j}^{I I I}-w_{n-1, j}^{I V} \geq a_{n j}^{(U, n)} \text { for } j \in N \\
& \sum_{\left\{i: j \in N_{i}\right\}} b_{n i j}^{(L, n)} \cdot w_{n i}^{I}+\sum_{\left\{i: j \in P_{i}\right\}} b_{n i j}^{(U, n)} \cdot w_{n i}^{I I}-w_{n j}^{I I I}+w_{n-1, j}^{V} \geq a_{n j}^{(L, n)} \text { for } j \in N \\
& \sum_{\left\{i: j \in N_{i}\right\}} b_{n i j}^{(L, n)} \cdot w_{n i}^{I}+\sum_{\left\{i: j \in P_{i}\right\}} b_{n i j}^{(U, n)} \cdot w_{n i}^{I I}-w_{n j}^{I I I}+w_{n-1, j}^{V} \geq a_{n j}^{(U, n)} \text { for } j \in P \\
& \mathbf{w}_{l}^{I}, \mathbf{w}_{l}^{I I} \in \mathbb{R}_{+}^{p} \text { and } \mathbf{w}_{l}^{I I I} \in \mathbb{R}_{+}^{q} \text { for } l=1, \cdots, n ;
\end{aligned}
$$




$$
\mathbf{w}_{l}^{I V}, \mathbf{w}_{l}^{V} \in \mathbb{R}_{+}^{q} \text { for } l=1, \cdots, n-1,
$$

where

$$
\mathbf{w}_{l}^{I}=\left(w_{l 1}^{I}, \cdots, w_{l p}^{I}\right) \text { and } \mathbf{w}_{l}^{I I}=\left(w_{l 1}^{I I}, \cdots, w_{l p}^{I I}\right)
$$

and

$$
\mathbf{w}_{l}^{I I I}=\left(w_{l 1}^{I I I}, \cdots, w_{l q}^{I I I}\right), \quad \mathbf{w}_{l}^{I V}=\left(w_{l 1}^{I V}, \ldots, w_{l q}^{I V}\right) \text { and } \mathbf{w}_{l}^{V}=\left(w_{l 1}^{V}, \cdots, w_{l q}^{V}\right) .
$$

Then

$$
\mathbf{w}_{l}^{B}=\left(\mathbf{w}_{l}^{I}, \mathbf{w}_{l}^{I I}\right), \quad \mathbf{w}_{l}^{A}=\mathbf{w}_{l}^{I I I} \text { and } \mathbf{w}_{l}^{C}=\left(\mathbf{w}_{l}^{I V}, \mathbf{w}_{l}^{V}\right) .
$$

We write

$$
\mathbf{w}^{B}=\left(\mathbf{w}_{1}^{B}, \cdots, \mathbf{w}_{n}^{B}\right), \quad \mathbf{w}^{A}=\left(\mathbf{w}_{1}^{A}, \cdots, \mathbf{w}_{n}^{A}\right) \text { and } \mathbf{w}^{C}=\left(\mathbf{w}_{1}^{C}, \cdots, \mathbf{w}_{n}^{C}\right) .
$$

The feasible solution of problem $\left(\mathrm{D}_{n}\right)$ is denoted by $\mathbf{w}=\left(\mathbf{w}^{B}, \mathbf{w}^{A}, \mathbf{w}^{C}\right)$.

Since $c_{l i}^{(L, n)}$ and $c_{l i}^{(U, n)}$ are nonnegative for $i=1, \cdots p$ and $l=1, \cdots, n$, it is clear to see that the zero vector $\mathbf{z}=\mathbf{0}$ is a feasible solution of problem $\left(\mathrm{P}_{n}\right)$, i.e., $V\left(\mathrm{P}_{n}\right) \geq 0$. The following result presents the feasibility and the bound of optimal objective value for dual problem $\left(\mathrm{D}_{n}\right)$, which will be used to design the computational procedure.

Proposition 4. For $l=1, \cdots, n$, we adopt the following notations:

$$
\begin{aligned}
\tau_{l}^{(L, n)} & =\max _{j=1, \cdots, q} a_{l j}^{(L, n)} \text { and } \tau_{l}^{(U, n)}=\max _{j=1, \cdots, q} a_{l j}^{(U, n)} \\
v_{l}^{(P N, n)} & =\min _{j=1, \cdots, q}\left\{\sum_{\left\{i: j \in P_{i}\right\}} b_{l i j}^{(L, n)}+\sum_{\left\{i: j \in N_{i}\right\}} b_{l i j}^{(U, n)}\right\} \\
v_{l}^{(N P, n)} & =\min _{j=1, \cdots, q}\left\{\sum_{\left\{i: j \in N_{i}\right\}} b_{l i j}^{(L, n)}+\sum_{\left\{i: j \in P_{i}\right\}} b_{l i j}^{(U, n)}\right\} .
\end{aligned}
$$

For $l=1, \cdots$, and $i=1, \cdots, p$, we define

$$
w_{l i}^{(I, n)}=w_{l i}^{(I I, n)}=\max \left\{\frac{\tau_{l}^{(L, n)}}{v_{l}^{(P N, n)},}, \frac{\tau_{l}^{(L, n)}}{v_{l}^{(N P, n)}}, \frac{\tau_{l}^{(U, n)}}{v_{l}^{(P N, n)}}, \frac{\tau_{l}^{(U, n)}}{v_{l}^{(N P, n)}}\right\} .
$$

For $l=1, \cdots$, n and $j=1, \cdots, q$, we define

$$
w_{l j}^{(I I I, n)}=0 .
$$

For $l=1, \cdots, n-1$ and $j=1, \cdots, q$, we define

$$
w_{l j}^{(I V, n)}=w_{l j}^{(V, n)}=0 .
$$

Then $\mathbf{w}^{(n)}=\left(\mathbf{w}^{(n, B)}, \mathbf{w}^{(n, A)}, \mathbf{w}^{(n, C)}\right)$ with

$$
\mathbf{w}_{l}^{(n, B)}=\left(\mathbf{w}_{l}^{(I, n)}, \mathbf{w}_{l}^{(I I, n)}\right), \mathbf{w}_{l}^{(n, A)}=\mathbf{w}_{l}^{(I I I, n)} \text { and } \mathbf{w}_{l}^{(n, C)}=\left(\mathbf{w}_{l}^{(I V, n)}, \mathbf{w}_{l}^{(V, n)}\right)
$$

and

$$
\mathbf{w}^{(n, B)}=\left(\mathbf{w}_{1}^{(n, B)}, \cdots, \mathbf{w}_{n}^{(n, B)}\right), \mathbf{w}^{(n, A)}=\left(\mathbf{w}_{1}^{(n, A)}, \ldots, \mathbf{w}_{n}^{(n, A)}\right) \text { and } \mathbf{w}^{(n, C)}=\left(\mathbf{w}_{1}^{(n, C)}, \cdots, \mathbf{w}_{n}^{(n, C)}\right) .
$$


is a feasible solution of problem $\left(\mathrm{D}_{n}\right)$. In other words, the dual problem $\left(\mathrm{D}_{n}\right)$ is feasible for each $n \in \mathbb{N}$. Moreover we have

$$
w_{l i}^{(I, n)}=w_{l i}^{(I I, n)} \leq \max \left\{\frac{\tau^{L}}{\sigma^{P N}}, \frac{\tau^{L}}{\sigma^{N P}}, \frac{\tau^{U}}{\sigma^{P N}}, \frac{\tau^{U}}{\sigma^{N P}}\right\} .
$$

for $l=1, \cdots, n$ and $i=1, \cdots, p$. We also have

$$
0 \leq V\left(\mathrm{D}_{n}\right) \leq p \cdot \max \left\{\frac{\tau^{L}}{\sigma^{P N}}, \frac{\tau^{L}}{\sigma^{N P}}, \frac{\tau^{U}}{\sigma^{P N}}, \frac{\tau^{U}}{\sigma^{N P}}\right\} \cdot\left(\mu^{L}+\mu^{U}\right) .
$$

Proof. From (15), (16) and (41), we see that $v_{l}^{(P N, n)} \geq \sigma^{P N}>0$ and $v_{l}^{(N P, n)} \geq \sigma^{N P}>0$ for $l=1, \cdots, n$. It means that $\mathbf{w}^{(n)}$ is well-defined and nonnegative. For $l=1, \cdots, n$, we define

$$
\mathfrak{w}_{l}=\max \left\{\frac{\tau_{l}^{(L, n)}}{v_{l}^{(P N, n)}}, \frac{\tau_{l}^{(L, n)}}{v_{l}^{(N P, n)}}, \frac{\tau_{l}^{(U, n)}}{v_{l}^{(P N, n)}}, \frac{\tau_{l}^{(U, n)}}{v_{l}^{(N P, n)}}\right\} .
$$

By substituting $\mathbf{w}^{(n)}$ into the constraints of $\left(\mathrm{D}_{n}\right)$ and using (57)-(59), we have, for $l=1, \cdots, n$,

$$
\begin{aligned}
& \sum_{\left\{i: j \in P_{i}\right\}} b_{l i j}^{(L, n)} \cdot w_{l i}^{(I, n)}+\sum_{\left\{i: j \in N_{i}\right\}} b_{l i j}^{(U, n)} \cdot w_{l i}^{(I I, n)}=\mathfrak{w}_{l} \cdot\left(\sum_{\left\{i: j \in P_{i}\right\}} b_{l i j}^{(L, n)}+\sum_{\left\{i: j \in N_{i}\right\}} b_{l i j}^{(U, n)}\right) \geq \mathfrak{w}_{l} \cdot v_{l}^{(P N, n)} \\
& \geq\left\{\begin{array}{l}
\frac{\tau_{l}^{(L, n)}}{v_{l}^{(P N, n)}} \cdot v_{l}^{(P N, n)}=\tau_{l}^{(L, n)} \geq a_{l j}^{(L, n)} \text { for } j \in P \\
\frac{\tau_{l}^{(U, n)}}{v_{l}^{(P N, n)}} \cdot v_{l}^{(P N, n)}=\tau_{l}^{(U, n)} \geq a_{l j}^{(U, n)} \text { for } j \in N
\end{array}\right\},
\end{aligned}
$$

and

$$
\begin{aligned}
& \sum_{\left\{i: j \in N_{i}\right\}} b_{l i j}^{(L, n)} \cdot w_{l i}^{(I, n)}+\sum_{\left\{i: j \in P_{i}\right\}} b_{l i j}^{(U, n)} \cdot w_{l i}^{(I I, n)}=\mathfrak{w}_{l} \cdot\left(\sum_{\left\{i: j \in N_{i}\right\}} b_{l i j}^{(L, n)}+\sum_{\left\{i: j \in P_{i}\right\}} b_{l i j}^{(U, n)}\right) \geq \mathfrak{w}_{l} \cdot v_{l}^{(N P, n)} \\
& \quad \geq\left\{\begin{array}{l}
\frac{\tau_{l}^{(L, n)}}{v_{l}^{(N P, n)}} \cdot v_{l}^{(N P, n)}=\tau_{l}^{(L, n)} \geq a_{l j}^{(L, n)} \text { for } j \in N \\
\frac{\tau_{l}^{(U, n)}}{v_{l}^{(N P, n)}} \cdot v_{l}^{(N P, n)}=\tau_{l}^{(U, n)} \geq a_{l j}^{(U, n)} \text { for } j \in P
\end{array}\right\} .
\end{aligned}
$$

This shows that $\mathbf{w}^{(n)}$ is indeed a feasible solution of problem $\left(\mathrm{D}_{n}\right)$. From (57), (36) and (39), we also see that

$$
\mathfrak{w}_{l} \leq \max \left\{\frac{\tau^{L}}{\sigma^{P N}}, \frac{\tau^{L}}{\sigma^{N P}}, \frac{\tau^{U}}{\sigma^{P N}}, \frac{\tau^{U}}{\sigma^{N P}}\right\} \text { for } l=1, \cdots, n .
$$

Using (37) and (40), the objective value of $\mathbf{w}^{(n)}$ is given by

$$
\begin{aligned}
& \sum_{i=1}^{p} \sum_{l=1}^{n} \frac{1}{n} \cdot c_{l i}^{(L, n)} \cdot w^{(I, n)}+\sum_{i=1}^{p} \sum_{l=1}^{n} \frac{1}{n} \cdot c_{l i}^{(U, n)} \cdot w^{(I I, n)} \\
& \quad \leq \sum_{i=1}^{p} \sum_{l=1}^{n} \frac{1}{n} \cdot \mu^{L} \cdot w^{(I, n)}+\sum_{i=1}^{p} \sum_{l=1}^{n} \frac{1}{n} \cdot \mu^{U} \cdot w^{(I I, n)}=\frac{p}{n} \cdot\left(\mu^{L}+\mu^{U}\right) \cdot \sum_{l=1}^{n} \mathfrak{w}_{l} .
\end{aligned}
$$

The inequality (60) is obvious, since the dual problem $\left(\mathrm{D}_{n}\right)$ is a minimization problem. This completes the proof. 
The following result presents the uniform boundedness of optimal solutions of primal and dual problems $\left(\mathrm{P}_{n}\right)$ and $\left(\mathrm{D}_{n}\right)$, which can be used to discuss the existence of optimal solution of problem SOP.

Proposition 5. The following statements hold true.

(i) Let $\mathbf{z}^{(* n)}=\left(\mathbf{z}_{1}^{(* n)}, \ldots, \mathbf{z}_{n}^{(* n)}\right)$ with $\mathbf{z}_{l}^{(* n)}=\left(\mathbf{z}_{l}^{(* L, n)}, \mathbf{z}_{l}^{(* U, n)}\right)$ for $l=1, \cdots, n$ be an optimal solution of primal problem $\left(\mathrm{P}_{n}\right)$. Suppose that $a_{l j}^{(L, n)}$ and $a_{l j}^{(U, n)}$ are nonnegative for $j=1, \cdots, q$ and $=1, \cdots, n$. Then $z_{l j}^{(* L, n)}$ and $z_{l j}^{(* U, n)}$ are uniformly bounded; that is to say, there exists a constant $\mathcal{K}_{1}$ that is independent of $n$ such that $z_{l j}^{(* L, n)} \leq \mathcal{K}_{1}$ and $z_{l j}^{(* U, n)} \leq \mathcal{K}_{1}$ for all $j=1, \cdots$, and $l=1, \cdots, n$.

(ii) Let $\mathbf{w}^{(* n)}=\left(\mathbf{w}^{(* n, B)}, \mathbf{w}^{(* n, A)}, \mathbf{w}^{(* n, C)}\right)$ with $\mathbf{w}_{l}^{(* n, B)}=\left(\mathbf{w}_{l}^{(* I, n)}, \mathbf{w}_{l}^{(* I I, n)}\right), \mathbf{w}_{l}^{(* n, A)}=\mathbf{w}_{l}^{(* I I I, n)}$, $\mathbf{w}_{l}^{(* n, C)}=\left(\mathbf{w}_{l}^{(* I V, n)}, \mathbf{w}_{l}^{(* V, n)}\right), \mathbf{w}^{(* n, B)}=\left(\mathbf{w}_{1}^{(* n, B)}, \cdots, \mathbf{w}_{n}^{(* n, B)}\right), \mathbf{w}^{(* n, A)}=\left(\mathbf{w}_{1}^{(* n, A)}, \ldots, \mathbf{w}_{n}^{(* n, A)}\right)$ and $\mathbf{w}^{(* n, C)}=\left(\mathbf{w}_{1}^{(* n, C)}, \ldots, \mathbf{w}_{n}^{(* n, C)}\right)$ be an optimal solution of dual problem $\left(\mathrm{D}_{n}\right)$. Then $w_{l i}^{(* I, n)}$ and $w_{l i}^{(* I I, n)}$ are uniformly bounded; that is to say, there exists a constant $\mathcal{K}_{2}$ that is independent of $n$ such that $w_{l i}^{(* I, n)} \leq \mathcal{K}_{2}$ and $w_{l i}^{(* I I, n)} \leq \mathcal{K}_{2}$ for all $i=1, \cdots, p$ and $l=1, \cdots, n$.

Proof. According to the strong duality theorem for linear programming problems, we first have $V\left(\mathrm{P}_{n}\right)=V\left(\mathrm{D}_{n}\right)$. Since $\mathbf{z}=\mathbf{0}$ is a feasible solution of primal problem $\left(\mathrm{P}_{n}\right)$, i.e., $V\left(\mathrm{P}_{n}\right) \geq 0$, using (60), we obtain

$$
0 \leq V\left(\mathrm{P}_{n}\right)=V\left(\mathrm{D}_{n}\right) \leq p \cdot \max \left\{\frac{\tau^{L}}{\sigma^{P N}}, \frac{\tau^{L}}{\sigma^{N P}}, \frac{\tau^{U}}{\sigma^{P N}}, \frac{\tau^{U}}{\sigma^{N P}}\right\} \cdot\left(\mu^{L}+\mu^{U}\right) .
$$

Since the objective values of $\mathbf{z}^{(* n)}$ and $\mathbf{w}^{(* n)}$ are $V\left(\mathrm{P}_{n}\right)$ and $V\left(\mathrm{D}_{n}\right)$, respectively, using (61) and the nonnegativity, it is clear to see that $z_{l j}^{(* L, n)}$ and $z_{l j}^{(* U, n)}$ must be bounded by some constant $\mathcal{K}_{1}$, and that $w_{l i}^{(* I, n)}$ and $w_{l i}^{(* I I, n)}$ must be bounded by some constant $\mathcal{K}_{2}$, where the constants $\mathcal{K}_{1}$ and $\mathcal{K}_{2}$ are independent of $n$. This completes the proof.

Let $\mathbf{z}^{(n)}=\left(\mathbf{z}_{1}^{(n)}, \ldots, \mathbf{z}_{n}^{(n)}\right)$ with $\mathbf{z}_{l}^{(n)}=\left(\mathbf{z}_{l}^{(L, n)}, \mathbf{z}_{l}^{(U, n)}\right)$ for $l=1, \cdots, n$ be a feasible solution of problem $\left(\mathrm{P}_{n}\right)$. For $j=1, \cdots, q$, we define the following real-valued functions:

$$
x_{j}^{(L, n)}(t)=\left\{\begin{array}{ll}
z_{l j}^{(L, n)} & \text { if } t \in F_{l}^{(n)}=\left[\frac{l-1}{n}, \frac{l}{n}\right) \text { for } l=1, \cdots, n \\
z_{n j}^{(L, n)} & \text { if } t=1
\end{array},\right.
$$

and

$$
x_{j}^{(U, n)}(t)= \begin{cases}z_{l j}^{(U, n)} & \text { if } t \in F_{l}^{(n)}=\left[\frac{l-1}{n}, \frac{l}{n}\right) \text { for } l=1, \cdots, n \\ z_{n j}^{(U, n)} & \text { if } t=1 .\end{cases}
$$

Then the feasibility is shown below.

Proposition 6. The vector-valued function $\mathbf{x}^{(n)}=\left(\mathbf{x}^{(L, n)}, \mathbf{x}^{(U, n)}\right)$ with component functions defined in (62) and (63) is a feasible solution of problem SOP.

Proof. Since $\mathbf{z}^{(n)}$ is a feasible solution of problem $\left(P_{n}\right)$, it follows that

$$
\begin{gathered}
\sum_{\left\{j: j \in P_{i}\right\}} b_{l i j}^{(L, n)} \cdot z_{l j}^{(L, n)}+\sum_{\left\{j: j \in N_{i}\right\}} b_{l i j}^{(L, n)} \cdot z_{l j}^{(U, n)} \leq c_{l i}^{(L, n)} \text { for } l=1, \cdots, n \text { and } i=1, \cdots, p ; \\
\sum_{\left\{j: j \in N_{i}\right\}} b_{l i j}^{(U, n)} \cdot z_{l j}^{(L, n)}+\sum_{\left\{j: j \in P_{i}\right\}} b_{l i j}^{(U, n)} \cdot z_{l j}^{(U, n)} \leq c_{l i}^{(U, n)} \text { for } l=1, \cdots, n \text { and } i=1, \cdots, p ; \\
z_{l j}^{(U, n)} \geq z_{l j}^{(L, n)} \text { for } j=1, \cdots, q \text { and } l=1, \cdots, n ;
\end{gathered}
$$




$$
\begin{aligned}
& z_{l j}^{(L, n)} \leq z_{l+1, j}^{(L, n)} \text { for } j=1, \cdots, q \text { and } l=1, \cdots, n-1 ; \\
& z_{l j}^{(U, n)} \geq z_{l+1, j}^{(U, n)} \text { for } j=1, \cdots, q \text { and } l=1, \cdots, n-1 .
\end{aligned}
$$

We consider the following cases.

- $\quad$ Suppose that $t \in F_{l}^{(n)}$ for $l=1, \ldots, n$. Then we have

$$
\begin{aligned}
& \sum_{\left\{j: j \in P_{i}\right\}} b_{i j}^{L}(t) \cdot x_{j}^{(L, n)}(t)+\sum_{\left\{j: j \in N_{i}\right\}} b_{i j}^{L}(t) \cdot x_{j}^{(U, n)}(t) \\
& =\sum_{\left\{j: j \in P_{i}\right\}} b_{i j}^{L}(t) \cdot z_{l j}^{(L, n)}+\sum_{\left\{j: j \in N_{i}\right\}} b_{i j}^{L}(t) \cdot z_{l j}^{(U, n)} \\
& \leq \sum_{\left\{j: j \in P_{i}\right\}} b_{l i j}^{(L, n)} \cdot z_{l j}^{(L, n)}+\sum_{\left\{j: j \in N_{i}\right\}} b_{l i j}^{(L, n)} \cdot z_{l j}^{(U, n)} \text { (by (41)) } \\
& \leq c_{l i}^{(L, n)}(\text { by (64)) } \\
& \leq c_{i}^{L}(t)(\text { by }(40)),
\end{aligned}
$$

and, using (65), we also have

$$
\begin{aligned}
& \sum_{\left\{j: j \in N_{i}\right\}} b_{i j}^{U}(t) \cdot x_{j}^{(L, n)}(t)+\sum_{\left\{j: j \in P_{i}\right\}} b_{i j}^{U}(t) \cdot x_{j}^{(U, n)}(t) \\
& =\sum_{\left\{j: j \in N_{i}\right\}} b_{i j}^{U}(t) \cdot z_{l j}^{(L, n)}+\sum_{\left\{j: j \in P_{i}\right\}} b_{i j}^{U}(t) \cdot z_{l j}^{(U, n)} \\
& \leq \sum_{\left\{j: j \in N_{i}\right\}} b_{l i j}^{(U, n)} \cdot z_{l j}^{(L, n)}+\sum_{\left\{j: j \in P_{i}\right\}} b_{l i j}^{(U, n)} \cdot z_{l j}^{(U, n)} \leq c_{l i}^{(U, n)} \leq c_{i}^{U}(t) .
\end{aligned}
$$

Suppose that $t=1$. Then

$$
\begin{aligned}
& \sum_{\left\{j: j \in P_{i}\right\}} b_{i j}^{L}(1) \cdot x_{j}^{(L, n)}(1)+\sum_{\left\{j: j \in N_{i}\right\}} b_{i j}^{L}(1) \cdot x_{j}^{(U, n)}(1) \\
& =\sum_{\left\{j: j \in P_{i}\right\}} b_{i j}^{L}(1) \cdot z_{n j}^{(L, n)}+\sum_{\left\{j: j \in N_{i}\right\}} b_{i j}^{L}(1) \cdot z_{n j}^{(U, n)} \\
& \leq \sum_{\left\{j: j \in P_{i}\right\}} b_{n i j}^{(L, n)} \cdot z_{n j}^{(L, n)}+\sum_{\left\{j: j \in N_{i}\right\}} b_{n i j}^{(L, n)} \cdot z_{n j}^{(U, n)} \leq c_{n i}^{(L, n)} \leq c_{i}^{L}(1),
\end{aligned}
$$

and

$$
\begin{aligned}
& \sum_{\left\{j: j \in N_{i}\right\}} b_{i j}^{U}(1) \cdot x_{j}^{(L, n)}(1)+\sum_{\left\{j: j \in P_{i}\right\}} b_{i j}^{U}(1) \cdot x_{j}^{(U, n)}(1) \\
& =\sum_{\left\{j: j \in N_{i}\right\}} b_{i j}^{U}(1) \cdot z_{n j}^{(L, n)}+\sum_{\left\{j: j \in P_{i}\right\}} b_{i j}^{U}(1) \cdot z_{n j}^{(U, n)} \\
& \leq \sum_{\left\{j: j \in N_{i}\right\}} b_{n i j}^{(U, n)} \cdot z_{n j}^{(L, n)}+\sum_{\left\{j: j \in P_{i}\right\}} b_{n i j}^{(U, n)} \cdot z_{n j}^{(U, n)} \leq c_{n i}^{(U, n)} \leq c_{i}^{U}(1) .
\end{aligned}
$$

- $\quad$ Suppose that $t \in F_{l}^{(n)}$ for $l=1, \cdots, n$. Then, using (66), we have

$$
x_{j}^{L}(t)=z_{l j}^{(L, n)} \leq z_{l j}^{(U, n)}=x_{j}^{U}(t) .
$$


Suppose that $t=1$. Then we also have

$$
x_{j}^{L}(1)=z_{n j}^{(L, n)} \leq z_{n j}^{(U, n)}=x_{j}^{U}(1) .
$$

This shows that $x_{j}^{U}(t) \geq x_{j}^{L}(t)$ for $j=1, \cdots, q$ and $t \in[0,1]$.

- $\quad$ Suppose that $t_{1} \in F_{l}^{(n)}$ and $t_{2} \in F_{l+1}^{(n)}$ for $l=1, \cdots, n-1$, i.e., $t_{1}<t_{2}$. Using (67), we have

$$
x_{j}^{L}\left(t_{1}\right)=z_{l j}^{(L, n)} \leq z_{l+1, j}^{(L, n)}=x_{j}^{L}\left(t_{2}\right) .
$$

Suppose that $t_{1} \in F_{n-1}^{(n)}$ and $t_{2}=1$. Then

$$
x_{j}^{L}\left(t_{1}\right)=z_{n-1, j}^{(L, n)} \leq z_{n j}^{(L, n)}=x_{j}^{L}(1)=x_{j}^{L}\left(t_{2}\right) .
$$

Suppose that $t_{1}, t_{2} \in F_{l}^{(n)}$ for $l=1, \cdots, n$ with $t_{1}<t_{2}$, or that $t_{1} \in F_{n}^{(n)}$ and $t_{2}=1$. Then it is clear that

$$
x_{j}^{L}\left(t_{1}\right)=z_{l j}^{(L, n)}=x_{j}^{L}\left(t_{2}\right) .
$$

This shows that $x_{j}^{L}$ is an increasing function on $[0,1]$.

- Suppose that $t_{1} \in F_{l}^{(n)}$ and $t_{2} \in F_{l+1}^{(n)}$ for $l=1, \cdots, n-1$, i.e., $t_{1}<t_{2}$. Using (68), we have

$$
x_{j}^{U}\left(t_{1}\right)=z_{l j}^{(U, n)} \geq z_{l+1, j}^{(U, n)}=x_{j}^{U}\left(t_{2}\right) .
$$

Suppose that $t_{1} \in F_{n-1}^{(n)}$ and $t_{2}=1$. Then

$$
x_{j}^{U}\left(t_{1}\right)=z_{n-1, j}^{(U, n)} \geq z_{n j}^{(U, n)}=x_{j}^{U}(1)=x_{j}^{U}\left(t_{2}\right) .
$$

Suppose that $t_{1}, t_{2} \in F_{l}^{(n)}$ for $l=1, \cdots, n$ with $t_{1}<t_{2}$, or that $t_{1} \in F_{n}^{(n)}$ and $t_{2}=1$. Then it is clear that

$$
x_{j}^{U}\left(t_{1}\right)=z_{l j}^{(U, n)}=x_{j}^{U}\left(t_{2}\right) .
$$

This shows that $x_{j}^{U}$ is a decreasing function on $[0,1]$.

Therefore we conclude that $\mathbf{x}^{(n)}=\left(\mathbf{x}^{(L, n)}, \mathbf{x}^{(U, n)}\right)$ is indeed a feasible solution of problem SOP. This completes the proof.

\section{Error Estimation}

In the sequel, we consider $\delta=1 / n$ in problems $\left(\mathrm{SOP}_{\delta}\right)$ and $\left(\mathrm{DSOP}_{\delta}\right)$. In this case, we also write $\left(\mathrm{SOP}_{\delta}\right)=\left(\mathrm{SOP}_{n}\right)$ and $\left(\mathrm{DSOP}_{\delta}\right)=\left(\mathrm{DSOP}_{n}\right)$. By rewriting problem $\left(\mathrm{DSOP}_{n}\right)$, we first note that $[0, \delta)=[0,1 / n)$,

$$
[\delta, 1-\delta)=[1 / n, 1-1 / n)=\bigcup_{l=2}^{n-1} F_{l} \text { and }[1-\delta, 1]=[1-1 / n, 1]=F_{n}^{(n)} \cup\{1\}=\bar{E}_{n}^{(n)} .
$$

Then the dual problem $\left(\mathrm{DSOP}_{n}\right)$ has the following form:

$$
\left(\mathrm{DSOP}_{n}\right) \quad \min \sum_{i=1}^{p} \int_{0}^{1} c_{i}^{L}(t) \cdot y_{i}^{I}(t) d t+\sum_{i=1}^{p} \int_{0}^{1} c_{i}^{U}(t) \cdot y_{i}^{I I}(t) d t
$$




$$
\begin{aligned}
& \text { subject to } \sum_{\left\{i: j \in P_{i}\right\}} b_{i j}^{L}(t) \cdot y_{i}^{I}(t)+\sum_{\left\{i: j \in N_{i}\right\}} b_{i j}^{U}(t) \cdot y_{i}^{I I}(t)+y_{j}^{I I I}(t)+y_{j}^{I V}(t) \geq a_{j}^{L}(t) \\
& \text { for } t \in F_{1}^{(n)}=[0, \delta)=[0,1 / n) \text { and } j \in P ; \\
& \sum_{\left\{i: j \in P_{i}\right\}} b_{i j}^{L}(t) \cdot y_{i}^{I}(t)+\sum_{\left\{i: j \in N_{i}\right\}} b_{i j}^{U}(t) \cdot y_{i}^{I I}(t)+y_{j}^{I I I}(t)+y_{j}^{I V}(t) \geq a_{j}^{U}(t) \\
& \text { for } t \in F_{1}^{(n)}=[0, \delta)=[0,1 / n) \text { and } j \in N ; \\
& \sum_{\left\{i: j \in N_{i}\right\}} b_{i j}^{L}(t) \cdot y_{i}^{I}(t)+\sum_{\left\{i: j \in P_{i}\right\}} b_{i j}^{U}(t) \cdot y_{i}^{I I}(t)-y_{j}^{I I I}(t)-y_{j}^{V}(t) \geq a_{j}^{L}(t) \\
& \text { for } t \in F_{1}^{(n)} \text { and } j \in N \text {; } \\
& \sum_{\left\{i: j \in N_{i}\right\}} b_{i j}^{L}(t) \cdot y_{i}^{I}(t)+\sum_{\left\{i: j \in P_{i}\right\}} b_{i j}^{U}(t) \cdot y_{i}^{I I}(t)-y_{j}^{I I I}(t)-y_{j}^{V}(t) \geq a_{j}^{U}(t) \\
& \text { for } t \in F_{1}^{(n)} \text { and } j \in P \text {; } \\
& \sum_{\left\{i: j \in P_{i}\right\}} b_{i j}^{L}(t) \cdot y_{i}^{I}(t)+\sum_{\left\{i: j \in N_{i}\right\}} b_{i j}^{U}(t) \cdot y_{i}^{I I}(t)+y_{j}^{I I I}(t) \\
& +y_{j}^{I V}(t)-y_{j}^{I V}\left(t-\frac{1}{n}\right) \geq a_{j}^{L}(t) \text { for } t \in F_{l}^{(n)}, l=2, \cdots, n-1 \text { and } j \in P ; \\
& \sum_{\left\{i: j \in P_{i}\right\}} b_{i j}^{L}(t) \cdot y_{i}^{I}(t)+\sum_{\left\{i: j \in N_{i}\right\}} b_{i j}^{U}(t) \cdot y_{i}^{I I}(t)+y_{j}^{I I I}(t) \\
& +y_{j}^{I V}(t)-y_{j}^{I V}\left(t-\frac{1}{n}\right) \geq a_{j}^{U}(t) \text { for } t \in F_{l}^{(n)}, l=2, \cdots, n-1 \text { and } j \in N ; \\
& \sum_{\left\{i: j \in N_{i}\right\}} b_{i j}^{L}(t) \cdot y_{i}^{I}(t)+\sum_{\left\{i: j \in P_{i}\right\}} b_{i j}^{U}(t) \cdot y_{i}^{I I}(t)-y_{j}^{I I I}(t) \\
& -y_{j}^{V}(t)+y_{j}^{V}\left(t-\frac{1}{n}\right) \geq a_{j}^{L}(t) \text { for } t \in F_{l}^{(n)}, l=2, \cdots, n-1 \text { and } j \in N ; \\
& \sum_{\left\{i: j \in N_{i}\right\}} b_{i j}^{L}(t) \cdot y_{i}^{I}(t)+\sum_{\left\{i: j \in P_{i}\right\}} b_{i j}^{U}(t) \cdot y_{i}^{I I}(t)-y_{j}^{I I I}(t) \\
& -y_{j}^{V}(t)+y_{j}^{V}\left(t-\frac{1}{n}\right) \geq a_{j}^{U}(t) \text { for } t \in F_{l}^{(n)}, l=2, \cdots, n-1 \text { and } j \in P ; \\
& \sum_{\left\{i: j \in P_{i}\right\}} b_{i j}^{L}(t) \cdot y_{i}^{I}(t)+\sum_{\left\{i: j \in N_{i}\right\}} b_{i j}^{U}(t) \cdot y_{i}^{I I}(t)+y_{j}^{I I I}(t)-y_{j}^{I V}\left(t-\frac{1}{n}\right) \geq a_{j}^{L}(t) \\
& \text { for } t \in F_{n}^{(n)} \cup\{1\}=[1-\delta, 1]=[1-1 / n, 1] \text { and } j \in P \text {; }
\end{aligned}
$$




$$
\begin{aligned}
& \sum_{\left\{i: j \in P_{i}\right\}} b_{i j}^{L}(t) \cdot y_{i}^{I}(t)+\sum_{\left\{i: j \in N_{i}\right\}} b_{i j}^{U}(t) \cdot y_{i}^{I I}(t)+y_{j}^{I I I}(t)-y_{j}^{I V}\left(t-\frac{1}{n}\right) \geq a_{j}^{U}(t) \\
& \text { for } t \in F_{n}^{(n)} \cup\{1\}=[1-\delta, 1]=[1-1 / n, 1] \text { and } j \in N ; \\
& \sum_{\left\{i: j \in N_{i}\right\}} b_{i j}^{L}(t) \cdot y_{i}^{I}(t)+\sum_{\left\{i: j \in P_{i}\right\}} b_{i j}^{U}(t) \cdot y_{i}^{I I}(t)-y_{j}^{I I I}(t)+y_{j}^{V}\left(t-\frac{1}{n}\right) \geq a_{j}^{L}(t) \\
& \quad \text { for } t \in F_{n}^{(n)} \cup\{1\}=\bar{E}_{n}^{(n)} \text { and } j \in N ; \\
& \sum_{\left\{i: j \in N_{i}\right\}} b_{i j}^{L}(t) \cdot y_{i}^{I}(t)+\sum_{\left\{i: j \in P_{i}\right\}} b_{i j}^{U}(t) \cdot y_{i}^{I I}(t)-y_{j}^{I I I}(t)+y_{j}^{V}\left(t-\frac{1}{n}\right) \geq a_{j}^{U}(t) \\
& \quad \text { for } t \in F_{n}^{(n)} \cup\{1\}=\bar{E}_{n}^{(n)} \text { and } j \in P ; \\
& y_{i}^{I}(t), y_{i}^{I I}(t) \geq 0 \text { for } t \in[0,1] \text { and } i=1, \cdots, p ; \\
& y_{j}^{I I I}(t), y_{j}^{I V}(t), y_{j}^{V}(t) \geq 0 \text { for } t \in[0,1] \text { and } j=1, \cdots, q .
\end{aligned}
$$

Let $\mathbf{z}^{(* n)}$ be an optimal solution of problem $\left(\mathrm{P}_{n}\right)$. Then, according to Proposition 6, we see that $\mathbf{x}^{(n)}$ constructed from $\mathbf{z}^{(* n)}$ is a feasible solution of problem SOP Therefore we obtain

$$
\begin{aligned}
V(\mathrm{SOP}) & \geq \sum_{j=1}^{q} \int_{0}^{1} a_{j}^{(L, n)}(t) \cdot x_{j}^{(L, n)}(t) d t+\sum_{j=1}^{q} \int_{0}^{1} a_{j}^{(U, n)}(t) \cdot x_{j}^{(U, n)}(t) d t \text { (by Proposition 6) } \\
& =\sum_{j=1}^{q} \sum_{l=1}^{n} \int_{F_{l}^{(n)}} a_{j}^{(L, n)}(t) \cdot x_{j}^{(L, n)}(t) d t+\sum_{j=1}^{q} \sum_{l=1}^{n} \int_{F_{l}^{(n)}} a_{j}^{(U, n)}(t) \cdot x_{j}^{(U, n)}(t) d t \\
& =\sum_{j=1}^{q} \sum_{l=1}^{n} \int_{F_{l}^{(n)}} a_{l j}^{(L, n)} \cdot z_{l j}^{(* L, n)} d t+\sum_{j=1}^{q} \sum_{l=1}^{n} \int_{F_{l}^{(n)}} a_{l j}^{(U, n)} \cdot z_{l j}^{(* U, n)} d t \\
& =\sum_{j=1}^{q} \sum_{l=1}^{n} \frac{1}{n} \cdot a_{l j}^{(L, n)} \cdot z_{l j}^{(* L, n)}+\sum_{j=1}^{q} \sum_{l=1}^{n} \frac{1}{n} \cdot a_{l j}^{(U, n)} \cdot z_{l j}^{(* U, n)}=V\left(\mathrm{P}_{n}\right) .
\end{aligned}
$$

Using Theorem 2, it follows that

$$
V\left(\mathrm{DSOP}_{n}\right) \geq V\left(\mathrm{SOP}_{n}\right) \geq V(\mathrm{SOP}) \geq V\left(\mathrm{P}_{n}\right)=V\left(\mathrm{D}_{n}\right) .
$$

We plan to show that

$$
\limsup _{n \rightarrow \infty} V\left(\mathrm{D}_{n}\right)=\limsup _{n \rightarrow \infty} V\left(\mathrm{DSOP}_{n}\right)
$$

We first define some notations. Let $\mathbf{w}^{(* n)}$ be an optimal solution of problem $\left(\mathrm{D}_{n}\right)$. For $j=1, \cdots, q$, we define the real-valued functions $h_{l j}^{(P N, n)}$ and $h_{l j}^{(N P, n)}$ on $F_{l}^{(n)}$ for $l=1, \cdots, n-1$ and $h_{n j}^{(P N, n)}$ and $h_{n j}^{(N P, n)}$ on $F_{n}^{(n)} \cup\{1\}=\bar{E}_{n}^{(n)}$ by

$$
h_{l j}^{(P N, n)}(t)=\sum_{\left\{i: j \in P_{i}\right\}}\left[b_{l i j}^{(L, n)}-b_{i j}^{L}(t)\right] \cdot w_{l i}^{(* I, n)}+\sum_{\left\{i: j \in N_{i}\right\}}\left[b_{l i j}^{(U, n)}-b_{i j}^{U}(t)\right] \cdot w_{l i}^{(* I I, n)},
$$

and

$$
h_{l j}^{(N P, n)}(t)=\sum_{\left\{i: j \in N_{i}\right\}}\left[b_{l i j}^{(L, n)}-b_{i j}^{L}(t)\right] \cdot w_{l i}^{(* I, n)}+\sum_{\left\{i: j \in P_{i}\right\}}\left[b_{l i j}^{(U, n)}-b_{i j}^{U}(t)\right] \cdot w_{l i}^{(* I I, n)} .
$$


It is clear to see that the real-valued functions $h_{l j}^{(P N, n)}$ and $h_{l j}^{(N P, n)}$ are nonnegative for $l=1, \cdots, n$ and $j=1, \cdots, q$.

For $l=1, \cdots, n$, we define

$$
\begin{aligned}
& \pi_{l}^{(P N L, n)}=\left\{\begin{array}{ll}
\max _{j \in P} \sup _{t \in F_{l}^{(n)}}\left[h_{l j}^{(P N, n)}(t)+a_{j}^{L}(t)-a_{l j}^{(L, n)}\right], & \text { if } P \neq \varnothing \\
0, & \text { if } P=\varnothing
\end{array}\right\} \\
& \pi_{l}^{(P N U, n)}=\left\{\begin{array}{ll}
\max _{j \in N} \sup _{t \in F_{l}^{(n)}}\left[h_{l j}^{(P N, n)}(t)+a_{j}^{U}(t)-a_{l j}^{(U, n)}\right], & \text { if } N \neq \varnothing \\
0, & \text { if } N=\varnothing
\end{array}\right\} \\
& \pi_{l}^{(N P L, n)}=\left\{\begin{array}{ll}
\max _{j \in N} \sup _{t \in F_{l}^{(n)}}\left[h_{l j}^{(N P, n)}(t)+a_{j}^{L}(t)-a_{l j}^{(L, n)}\right], & \text { if } N \neq \varnothing \\
0, & \text { if } N=\varnothing
\end{array}\right\} \\
& \pi_{l}^{(N P U, n)}=\left\{\begin{array}{ll}
\max _{j \in P} \sup _{t \in F_{l}^{(n)}}\left[h_{l j}^{(N P, n)}(t)+a_{j}^{U}(t)-a_{l j}^{(U, n)}\right] & \text { if } P \neq \varnothing \\
0, & \text { if } P=\varnothing
\end{array}\right\} .
\end{aligned}
$$

We also define

$$
\pi_{l}^{(P N, n)}=\max \left\{\pi_{l}^{(P N L, n)}, \pi_{l}^{(P N U, n)}\right\} \text { and } \pi_{l}^{(N P, n)}=\max \left\{\pi_{l}^{(N P L, n)}, \pi_{l}^{(N P U, n)}\right\} .
$$

It is also clear to see that $\pi_{l}^{(P N, n)} \geq 0$ and $\pi_{l}^{(N P, n)} \geq 0$ for $l=1, \cdots, n$. We want to prove

$$
\lim _{n \rightarrow \infty} \pi_{l}^{(P N, n)}=0=\lim _{n \rightarrow \infty} \pi_{l}^{(N P, n)} .
$$

We first provide some useful lemmas.

Lemma 1. For $i=1, \cdots, p, j=1, \cdots, q$ and $l=1, \cdots, n$, we have

$$
\begin{aligned}
& \sup _{t \in F_{l}^{(n)}}\left[a_{j}^{L}(t)-a_{l j}^{(L, n)}\right] \rightarrow 0 \text { and } \sup _{t \in F_{l}^{(n)}}\left[a_{j}^{U}(t)-a_{l j}^{(U, n)}\right] \rightarrow 0 \text { as } n \rightarrow \infty \\
& \sup _{t \in F_{l}^{(n)}}\left[c_{i}^{L}(t)-c_{l i}^{(L, n)}\right] \rightarrow 0 \text { and } \sup _{t \in F_{l}^{(n)}}\left[c_{i}^{U}(t)-c_{l i}^{(U, n)}\right] \rightarrow 0 \text { as } n \rightarrow \infty \\
& \sup _{t \in F_{l}^{(n)}}\left[b_{l i j}^{(L, n)}-b_{i j}^{L}(t)\right] \rightarrow 0 \text { and } \sup _{t \in F_{l}^{(n)}}\left[b_{l i j}^{(U, n)}-b_{i j}^{U}(t)\right] \rightarrow 0 \text { as } n \rightarrow \infty .
\end{aligned}
$$

Proof. We see that $a_{j}^{L}$ is continuous on $F_{l}^{(n)}=\left[\frac{l-1}{n}, \frac{l}{n}\right)$. Let $\left\{\delta_{m}\right\}_{m=1}^{\infty}$ be a decreasing sequence such that it converges to zero and $\delta_{m}>0$ for all $m$, where $\delta_{1}$ is taken to satisfy $\delta_{1}<\frac{l}{n}-\frac{l-1}{n}$. Therefore we can define the compact interval

$$
E_{l m}^{(n)}=\left[\frac{l-1}{n}, \frac{l}{n}-\delta_{m}\right]
$$

Then we have

$$
F_{l}^{(n)}=\bigcup_{m=1}^{\infty} E_{l m}^{(n)} \text { and } E_{l m_{1}}^{(n)} \subseteq E_{l m_{2}}^{(n)} \text { for } m_{2}>m_{1}
$$


Since $E_{l m}^{(n)} \subset F_{l}^{(n)}$, it follows that $a_{j}^{L}$ is continuous on each compact interval $E_{l m}^{(n)}$, which also means that $a_{j}^{L}$ is uniformly continuous on each compact interval $E_{l m}^{(n)}$. Therefore, given any $\epsilon>0$, there exists $\delta>0$ such that $\left|t_{1}-t_{2}\right|<\delta$ implies

$$
\left|a_{j}^{L}\left(t_{1}\right)-a_{j}^{L}\left(t_{2}\right)\right|<\frac{\epsilon}{2} \text { for any } t_{1}, t_{2} \in E_{l m}^{(n)} .
$$

Since the length of each $F_{l}^{(n)}$ is $1 / n$, we can consider a sufficiently large $n_{0} \in \mathbb{N}$ such that $1 / n_{0}<\delta$. In this case, each length of $F_{l}^{(n)}$ for $l=1, \cdots, n$ is less than $\delta$ for $n \geq n_{0}$. In other words, if $n \geq n_{0}$, then (78) is satisfied for any $t_{1}, t_{2} \in E_{l m}^{(n)}$. We consider the following cases.

- Suppose that the infimum $a_{l j}^{(L, n)}$ is attained at $t^{(n *)} \in F_{l}^{(n)}$, i.e., $a_{l j}^{(L, n)}=a_{j}^{L}\left(t^{(n *)}\right)$. From (77), there exists $m^{*}$ such that $t^{(n *)} \in E_{l m^{*}}^{(n)}$. Now, given any $t \in F_{l}^{(n)}$, we see that $t \in E_{l m_{0}}^{(n)}$ for some $m_{0}$. Let $m=\max \left\{m_{0}, m^{*}\right\}$. From (77), it follows that $t, t^{(n *)} \in E_{l m}^{(n)}$. Then we have

$$
\left|a_{j}^{L}(t)-a_{l j}^{(L, n)}\right|=\left|a_{j}^{L}(t)-a_{j}^{L}\left(t^{(n *)}\right)\right|<\frac{\epsilon}{2}
$$

since the length of $E_{l m}^{(n)}$ is less than $\delta$, where $\epsilon$ is independent of $t$ because of the uniform continuity. - Suppose that the infimum $a_{l j}^{(L, n)}$ is not attained at any point in $F_{l}^{(n)}$. It means that $a_{l j}^{(L, n)}$ is increasing or decreasing on $F_{l}^{(n)}$. Since $a_{j}^{L}$ is continuous on $F_{l}^{(n)}$, it follows that the infimum $a_{l j}^{(L, n)}$ is either the righthand limit or lefthand limit given by

$$
a_{l j}^{(L, n)}=\lim _{t \rightarrow\left(\frac{l-1}{n}\right)+} a_{j}^{L}(t) \text { or } a_{l j}^{(L, n)}=\lim _{t \rightarrow\left(\frac{l}{n}\right)-} a_{j}^{L}(t) .
$$

Therefore, for sufficiently large $n$, i.e., the interval $F_{l}^{(n)}$ is sufficiently small such that its length $1 / n$ is less than $\delta$, using (79), we have

$$
\left|a_{j}^{L}(t)-a_{l j}^{(L, n)}\right|<\frac{\epsilon}{2}
$$

for all $t \in F_{l}^{(n)}$.

From the above two cases, since $a_{j}^{L}(t) \geq a_{l j}^{(L, n)}$ for all $t \in F_{l}^{(n)}$, we conclude that

$$
0 \leq \sup _{t \in F_{l}^{(n)}}\left[a_{j}^{L}(t)-a_{l j}^{(L, n)}\right] \leq \frac{\epsilon}{2}<\epsilon \text { for } l=1, \cdots, n .
$$

The other cases can be similarly obtained. This completes the proof.

Lemma 2. For each $l=1, \cdots, n$, we have

$$
\lim _{n \rightarrow \infty} \pi_{l}^{(P N, n)}=0=\lim _{n \rightarrow \infty} \pi_{l}^{(N P, n)} .
$$

Proof. We want to prove

$$
\sup _{t \in F_{l}^{(n)}}\left[h_{l j}^{(P N, n)}(t)+a_{j}^{L}(t)-a_{l j}^{(L, n)}\right] \rightarrow 0 \text { and } \sup _{t \in F_{l}^{(n)}}\left[h_{l j}^{(P N, n)}(t)+a_{j}^{U}(t)-a_{l j}^{(U, n)}\right] \rightarrow 0 \text { as } n \rightarrow \infty .
$$


Now we have

$$
\begin{aligned}
0 \leq & \sup _{t \in F_{l}^{(n)}}\left[h_{l j}^{(P N, n)}(t)+a_{j}^{L}(t)-a_{l j}^{(L, n)}\right] \\
\leq & \sum_{\left\{i: j \in P_{i}\right\}} w_{l i}^{(* I, n)} \cdot \sup _{t \in F_{l}^{(n)}}\left[b_{l i j}^{(L, n)}-b_{i j}^{L}(t)\right]+\sum_{\left\{i: j \in N_{i}\right\}} w_{l i}^{(* I I, n)} \cdot \sup _{t \in F_{l}^{(n)}}\left[b_{l i j}^{(U, n)}-b_{i j}^{U}(t)\right] \\
& +\sup _{t \in F_{l}^{(n)}}\left[a_{j}^{L}(t)-a_{l j}^{(L, n)}\right]
\end{aligned}
$$

and

$$
\begin{aligned}
0 \leq & \sup _{t \in F_{l}^{(n)}}\left[h_{l j}^{(P N, n)}(t)+a_{j}^{U}(t)-a_{l j}^{(U, n)}\right] \\
\leq & \sum_{\left\{i: j \in P_{i}\right\}} w_{l i}^{(* I, n)} \cdot \sup _{t \in F_{l}^{(n)}}\left[b_{l i j}^{(L, n)}-b_{i j}^{L}(t)\right]+\sum_{\left\{i: j \in N_{i}\right\}} w_{l i}^{(* I I, n)} \cdot \sup _{t \in F_{l}^{(n)}}\left[b_{l i j}^{(U, n)}-b_{i j}^{U}(t)\right] \\
& +\sup _{t \in F_{l}^{(n)}}\left[a_{j}^{U}(t)-a_{l j}^{(U, n)}\right] .
\end{aligned}
$$

Since the sequences $\left\{w_{l i}^{(* I, n)}\right\}_{n=1}^{\infty}$ and $\left\{w_{l i}^{(* I I, n)}\right\}_{n=1}^{\infty}$ are uniformly bounded by part (ii) of Proposition 5, using Lemma 1 , we obtain $\pi_{l}^{(P N, n)} \rightarrow 0$ as $n \rightarrow \infty$. We can similarly show that $\pi_{l}^{(N P, n)} \rightarrow 0$ as $n \rightarrow \infty$. This completes the proof.

For $l=1, \cdots, n$, we define

$$
\mathfrak{b}_{l}^{(P N, n)}=\min _{j=1, \cdots, q}\left\{\sum_{\left\{i: j \in P_{i}\right\}} \inf _{t \in \bar{E}_{l}^{(n)}} b_{i j}^{L}(t)+\sum_{\left\{i: j \in N_{i}\right\}} \inf _{t \in \bar{E}_{l}^{(n)}} b_{i j}^{U}(t)\right\}
$$

and

$$
\mathfrak{b}_{l}^{(N P, n)}=\min _{j=1, \cdots, q}\left\{\sum_{\left\{i: j \in N_{i}\right\}} \inf _{t \in \bar{E}_{l}^{(n)}} b_{i j}^{L}(t)+\sum_{\left\{i: j \in P_{i}\right\}} \inf _{t \in \bar{E}_{l}^{(n)}} b_{i j}^{U}(t)\right\} .
$$

From (15) and (16), we see that

$$
\mathfrak{b}_{l}^{(P N, n)} \geq \sigma^{P N}>0 \text { and } \mathfrak{b}_{l}^{(N P, n)} \geq \sigma^{N P}>0 \text { for } l=1, \cdots, n
$$

Since $b_{i j}^{L}$ is increasing on $[0,1]$ and $b_{i j}^{U}$ is decreasing on $[0,1]$, it follows that, for $l=1, \cdots, n$,

$$
\sum_{\left\{i: j \in P_{i}\right\}} \inf _{t \in \bar{E}_{l}^{(n)}} b_{i j}^{L}(t)=\sum_{\left\{i: j \in P_{i}\right\}} b_{i j}^{L}\left(\frac{l-1}{n}\right)
$$

and

$$
\sum_{\left\{i: j \in N_{i}\right\}} \inf _{t \in \bar{E}_{l}^{(n)}} b_{i j}^{U}(t)=\sum_{\left\{i: j \in N_{i}\right\}} b_{i j}^{U}\left(\frac{l}{n}\right)
$$

which says that

$$
\mathfrak{b}_{l}^{(P N, n)}=\min _{j=1, \cdots, q}\left\{\sum_{\left\{i: j \in P_{i}\right\}} b_{i j}^{L}\left(\frac{l-1}{n}\right)+\sum_{\left\{i: j \in N_{i}\right\}} b_{i j}^{U}\left(\frac{l}{n}\right)\right\} .
$$


We can similarly obtain

$$
\mathfrak{b}_{l}^{(N P, n)}=\min _{j=1, \cdots, q}\left\{\sum_{\left\{i: j \in N_{i}\right\}} b_{i j}^{L}\left(\frac{l-1}{n}\right)+\sum_{\left\{i: j \in P_{i}\right\}} b_{i j}^{U}\left(\frac{l}{n}\right)\right\} .
$$

We also define the real-valued functions $\mathfrak{v}^{(P N, n)}$ and $\mathfrak{v}^{(N P, n)}$ on $[0,1]$ by

$$
\mathfrak{v}^{(P N, n)}(t)= \begin{cases}\mathfrak{b}_{l}^{(P N, n),} & \text { if } t \in F_{l}^{(n)} \text { for } l=1, \cdots, n \\ \mathfrak{b}_{n}^{(P N, n),} & \text { if } t=1\end{cases}
$$

and

$$
\mathfrak{v}^{(N P, n)}(t)= \begin{cases}\mathfrak{b}_{l}^{(N P, n)}, & \text { if } t \in F_{l}^{(n)} \text { for } l=1, \cdots, n \\ \mathfrak{b}_{n}^{(N P, n)}, & \text { if } t=1 .\end{cases}
$$

From (80), it is clear to see that

$$
\mathfrak{v}^{(P N, n)}(t) \geq \sigma^{P N}>0 \text { and } \mathfrak{v}^{(N P, n)}(t) \geq \sigma^{N P}>0 \text { for } t \in[0,1] .
$$

Now we define the real numbers

$$
\kappa^{(P N, n)}=\max \left\{\max _{j \in P}\left\{h_{n j}^{(P N, n)}(1)+a_{j}^{L}(1)-a_{n j}^{(L, n)}\right\}, \max _{j \in N}\left\{h_{n j}^{(P N, n)}(1)+a_{j}^{U}(1)-a_{n j}^{(U, n)}\right\}\right\}
$$

and

$$
\kappa^{(N P, n)}=\max \left\{\max _{j \in N}\left\{h_{n j}^{(N P, n)}(1)+a_{j}^{L}(1)-a_{n j}^{(L, n)}\right\}, \max _{j \in P}\left\{h_{n j}^{(N P, n)}(1)+a_{j}^{U}(1)-a_{n j}^{(U, n)}\right\}\right\},
$$

where we assume

$$
\max _{j \in P}\left\{h_{n j}^{(P N, n)}(1)+a_{j}^{L}(1)-a_{n j}^{(L, n)}\right\}=0=\max _{j \in P}\left\{h_{n j}^{(N P, n)}(1)+a_{j}^{U}(1)-a_{n j}^{(U, n)}\right\} \text { for } P=\varnothing
$$

and

$$
\max _{j \in N}\left\{h_{n j}^{(P N, n)}(1)+a_{j}^{U}(1)-a_{n j}^{(U, n)}\right\}=0=\max _{j \in N}\left\{h_{n j}^{(N P, n)}(1)+a_{j}^{L}(1)-a_{n j}^{(L, n)}\right\} \text { for } N=\varnothing .
$$

We also define the real-valued functions $\mathfrak{p}^{(P N, n)}$ and $\mathfrak{p}^{(N P, n)}$ on $[0,1]$ by

$$
\mathfrak{p}^{(P N, n)}(t)= \begin{cases}\pi_{l}^{(P N, n),} & \text { if } t \in F_{l}^{(n)} \text { for } l=1, \cdots, n \\ \kappa^{(P N, n),}, & \text { if } t=1\end{cases}
$$

and

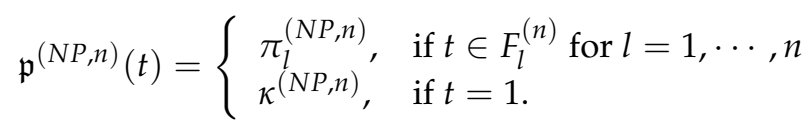

Using part (ii) of Proposition 5 and Lemma 1, we see that the sequences $\left\{h_{l j}^{(P N, n)}\right\}_{n=1}^{\infty}$ and $\left\{h_{l j}^{(N P, n)}\right\}_{n=1}^{\infty}$ of functions are uniformly bounded, which also say that the sequences $\left\{\pi_{l}^{(P N, n)}\right\}_{n=1}^{\infty}$, $\left\{\pi_{l}^{(N P, n)}\right\}_{n=1}^{\infty},\left\{\kappa^{(P N, n)}\right\}_{n=1}^{\infty}$ and $\left\{\kappa^{(N P, n)}\right\}_{n=1}^{\infty}$ of real numbers are bounded. Therefore there exist two constants $\mathfrak{c}^{P N}$ and $\mathfrak{c}^{N P}$ such that $\pi_{l}^{(P N, n)} \leq \mathfrak{c}^{P N}$ and $\pi_{l}^{(N P, n)} \leq \mathfrak{c}^{N P}$ for all $n \in \mathbb{N}$ and $l=1, \cdots, n$. Then

$$
\mathfrak{p}^{(P N, n)}(t) \leq \mathfrak{c}^{P N} \text { and } \mathfrak{p}^{(N P, n)}(t) \leq \mathfrak{c}^{N P} \text { for all } n \in \mathbb{N} \text { and } t \in[0,1] .
$$


We also define the real-valued functions $\mathfrak{f}^{(P N, n)}$ and $\mathfrak{f}^{(N P, n)}$ on $[0,1]$ by

$$
\mathfrak{f}^{(P N, n)}(t)=\frac{\mathfrak{p}^{(P N, n)}(t)}{\mathfrak{v}^{(P N, n)}(t)} \text { and } \mathfrak{f}^{(N P, n)}(t)=\frac{\mathfrak{p}^{(N P, n)}(t)}{\mathfrak{v}^{(N P, n)}(t)}
$$

It is clear to see that the real-valued functions $\mathfrak{f}^{(P N, n)}$ and $\mathfrak{f}^{(N P, n)}$ are nonnegative. The following lemma will be used for further discussion.

Lemma 3. For $l=1, \cdots, n, j=1, \cdots$, and $t \in F_{l}^{(n)}$, we have

$$
\mathfrak{f}^{(P N, n)}(t) \cdot\left(\sum_{\left\{i: j \in P_{i}\right\}} b_{i j}^{L}(t)+\sum_{\left\{i: j \in N_{i}\right\}} b_{i j}^{U}(t)\right) \geq\left\{\begin{array}{l}
h_{l j}^{(P N, n)}(t)+a_{j}^{L}(t)-a_{l j}^{(L, n)} \text { for } j \in P \\
h_{l j}^{(P N, n)}(t)+a_{j}^{U}(t)-a_{l j}^{(U, n)} \text { for } j \in N
\end{array}\right\}
$$

and

$$
\mathfrak{f}^{(N P, n)}(t) \cdot\left(\sum_{\left\{i: j \in N_{i}\right\}} b_{i j}^{L}(t)+\sum_{\left\{i: j \in P_{i}\right\}} b_{i j}^{U}(t)\right) \geq\left\{\begin{array}{l}
h_{l j}^{(N P, n)}(t)+a_{j}^{L}(t)-a_{l j}^{(L, n)} \text { for } j \in N \\
h_{l j}^{(N P, n)}(t)+a_{j}^{U}(t)-a_{l j}^{(U, n)} \text { for } j \in P
\end{array}\right\} .
$$

For $t=1$ and $j=1, \cdots, q$, we also have

$$
\mathfrak{f}^{(P N, n)}(1) \cdot\left(\sum_{\left\{i: j \in P_{i}\right\}} b_{i j}^{L}(1)+\sum_{\left\{i: j \in N_{i}\right\}} b_{i j}^{U}(1)\right) \geq\left\{\begin{array}{l}
h_{n j}^{(P N, n)}(1)+a_{j}^{L}(1)-a_{n j}^{(L, n)} \text { for } j \in P \\
h_{n j}^{(P N, n)}(1)+a_{j}^{U}(1)-a_{n j}^{(U, n)} \text { for } j \in N
\end{array}\right\}
$$

and

$$
\mathfrak{f}^{(N P, n)}(1) \cdot\left(\sum_{\left\{i: j \in N_{i}\right\}} b_{i j}^{L}(1)+\sum_{\left\{i: j \in P_{i}\right\}} b_{i j}^{U}(1)\right) \geq\left\{\begin{array}{l}
h_{n j}^{(N P, n)}(1)+a_{j}^{L}(1)-a_{n j}^{(L, n)} \text { for } j \in N \\
h_{n j}^{(N P, n)}(1)+a_{j}^{U}(1)-a_{n j}^{(U, n)} \text { for } j \in P
\end{array}\right\} .
$$

The sequences $\left\{\mathfrak{f}^{(P N, n)}\right\}_{n=1}^{\infty}$ and $\left\{\mathfrak{f}^{(N P, n)}\right\}_{n=1}^{\infty}$ of real-valued functions are uniformly bounded.

Proof. It suffices to consider the function $\mathfrak{f}^{(P N, n)}$. For $l=1, \cdots, n, j=1, \cdots, q$ and $t \in F_{l}^{(n)}$, using (76), we have

$$
\begin{aligned}
& \mathfrak{f}^{(P N, n)}(t) \cdot\left(\sum_{\left\{i: j \in P_{i}\right\}} b_{i j}^{L}(t)+\sum_{\left\{i: j \in N_{i}\right\}} b_{i j}^{U}(t)\right) \geq \mathfrak{f}^{(P N, n)}(t) \cdot \mathfrak{b}_{l}^{(P N, n)}=\mathfrak{f}^{(P N, n)}(t) \cdot \mathfrak{v}^{(P N, n)}(t) \\
& \quad=\mathfrak{p}^{(P N, n)}(t)=\pi_{l}^{(P N, n)} \geq\left\{\begin{array}{l}
\pi_{l}^{(P N L, n)} \geq h_{l j}^{(P N, n)}(t)+a_{j}^{L}(t)-a_{l j}^{(L, n)} \text { for } j \in P \\
\pi_{l}^{(P N U, n)} \geq h_{l j}^{(P N, n)}(t)+a_{j}^{U}(t)-a_{l j}^{(U, n)} \text { for } j \in N
\end{array}\right\} .
\end{aligned}
$$

For $t=1$, we also have

$$
\begin{gathered}
\mathfrak{f}^{(P N, n)}(1) \cdot\left(\sum_{\left\{i: j \in P_{i}\right\}} b_{i j}^{L}(1)+\sum_{\left\{i: j \in N_{i}\right\}} b_{i j}^{U}(1)\right) \geq \mathfrak{f}^{(P N, n)}(1) \cdot \mathfrak{b}_{n}^{(P N, n)}=\mathfrak{f}^{(P N, n)}(1) \cdot \mathfrak{v}^{(P N, n)}(1) \\
=\mathfrak{p}^{(P N, n)}(1)=\kappa^{(P N, n)} \geq\left\{\begin{array}{l}
h_{n j}^{(P N, n)}(1)+a_{j}^{L}(1)-a_{n j}^{(L, n)} \text { for } j \in P \\
h_{n j}^{(P N, n)}(1)+a_{j}^{U}(1)-a_{n j}^{(U, n)} \text { for } j \in N .
\end{array}\right\}
\end{gathered}
$$

From (83) and (86), it is obvious that the sequences $\left\{\mathfrak{f}^{(P N, n)}\right\}_{n=1}^{\infty}$ and $\left\{\mathfrak{f}^{(N P, n)}\right\}_{n=1}^{\infty}$ of real-valued functions are uniformly bounded. This completes the proof. 
Let $\mathbf{w}^{(* n)}$ be an optimal solution of problem $\left(\mathrm{D}_{n}\right)$. For $i=1, \cdots, p$ and $j=1, \cdots, q$, we define the following real-valued functions:

$$
\begin{aligned}
y_{i}^{(I, n)}(t)=\left\{\begin{array}{ll}
w_{l i}^{(* I, n)}+\max \left\{\mathfrak{f}^{(P N, n)}(t), \mathfrak{f}^{(N P, n)}(t)\right\}, & \text { if } t \in F_{l}^{(n)} \text { for } l=1, \cdots, n \\
w_{n i}^{(* I, n)}+\max \left\{\mathfrak{f}^{(P N, n)}(1), \mathfrak{f}^{(N P, n)}(1)\right\}, & \text { if } t=1
\end{array}\right\} ; \\
y_{i}^{(I I, n)}(t)=\left\{\begin{array}{ll}
w_{l i}^{(* I I, n)}+\max \left\{\mathfrak{f}^{(P N, n)}(t), \mathfrak{f}^{(N P, n)}(t)\right\}, & \text { if } t \in F_{l}^{(n)} \text { for } l=1, \cdots, n \\
w_{n i}^{(* I I, n)}+\max \left\{\mathfrak{f}^{(P N, n)}(1), \mathfrak{f}^{(N P, n)}(1)\right\}, & \text { if } t=1
\end{array}\right\} ; \\
y_{j}^{(I I I, n)}(t)=\left\{\begin{array}{ll}
w_{l j}^{(* I I, n)}, & \text { if } t \in F_{l}^{(n)} \text { for } l=1, \cdots, n \\
\bar{w}_{n j}^{(* I I I, n)}, & \text { if } t=1
\end{array}\right\} ; \\
y_{j}^{(I V, n)}(t)=\left\{\begin{array}{ll}
w_{l j}^{(* I V, n)}, & \text { if } t \in F_{l}^{(n)} \text { for } l=1, \cdots, n-1 \\
w_{n-1, j}^{(* I V, n)}, & \text { if } t \in F_{n}^{(n)} \cup\{1\}=\bar{E}_{n}^{(n)}
\end{array}\right\} ; \\
y_{j}^{(V, n)}(t)=\left\{\begin{array}{ll}
w_{l j}^{(* V, n),} & \text { if } t \in F_{l}^{(n)} \text { for } l=1, \cdots, n-1 \\
w_{n-1, j}^{(* V, n)}, & \text { if } t \in F_{n}^{(n)} \cup\{1\}=\bar{E}_{n}^{(n)}
\end{array}\right\} .
\end{aligned}
$$

Then we have the following feasibility.

Proposition 7. The vector-valued function

$$
\mathbf{y}^{(n)}(t)=\left(\mathbf{y}^{(I, n)}(t), \mathbf{y}^{(I I, n)}(t), \mathbf{y}^{(I I I, n)}(t), \mathbf{y}^{(I V, n)}(t), \mathbf{y}^{(V, n)}(t)\right)
$$

with component functions defined in (88)-(92) is a feasible solution of problem (DSOP $\left.{ }_{n}\right)$.

Proof. We are going to show that $\mathbf{y}^{(n)}$ satisfies all the constraints of problem $\left(\mathrm{DSOP}_{n}\right)$. For $j=1, \cdots, q$, we define the real-valued functions $g_{l j}^{(U, n)}$ on $F_{l}^{(n)}$ for $l=1, \cdots, n-1$ and $g_{n j}^{(U, n)}$ on $\bar{E}_{n}^{(n)}=F_{n}^{(n)} \cup\{1\}$ by

$$
\begin{aligned}
g_{l j}^{(N P, n)}(t)= & \sum_{\left\{i: j \in N_{i}\right\}}\left(b_{i j}^{L}(t)-b_{l i j}^{(L, n)}\right) \cdot w_{l i}^{(* I, n)}+\sum_{\left\{i: j \in P_{i}\right\}}\left(b_{i j}^{U}(t)-b_{l i j}^{(U, n)}\right) \cdot w_{l i}^{(* I, n)} \\
& +\mathfrak{f}^{(N P, n)}(t) \cdot\left(\sum_{\left\{i: j \in N_{i}\right\}} b_{i j}^{L}(t)+\sum_{\left\{i: j \in P_{i}\right\}} b_{i j}^{U}(t)\right) .
\end{aligned}
$$

Using Lemma 3, we have

$$
\begin{aligned}
g_{l j}^{(N P, n)}(t) & =-h_{l j}^{(N P, n)}(t)+\mathfrak{f}^{(N P, n)}(t) \cdot\left(\sum_{\left\{i: j \in N_{i}\right\}} b_{i j}^{L}(t)+\sum_{\left\{i: j \in P_{i}\right\}} b_{i j}^{U}(t)\right) \\
& \geq\left\{\begin{array}{l}
a_{j}^{L}(t)-a_{l j}^{(L, n)} \text { for } j \in N \\
a_{j}^{U}(t)-a_{l j}^{(U, n)} \text { for } j \in P
\end{array}\right\} .
\end{aligned}
$$

Since $1 / n$ is the length of each interval $F_{l}^{(n)}$, for $t \in F_{l}^{(n)}$ and $l=2, \cdots, n$, we see that $t-\frac{1}{n} \in F_{l-1}^{(n)}$. We consider the following cases. 
- $\quad$ For $l=2, \cdots, n-1$ and $t \in F_{l}^{(n)}$, i.e., $t-\frac{1}{n} \in F_{l-1}^{(n)}$, we obtain

$$
\begin{aligned}
\sum_{\left\{i: j \in N_{i}\right\}} b_{i j}^{L}(t) \cdot y_{i}^{(I, n)}(t)+\sum_{\left\{i: j \in P_{i}\right\}} b_{i j}^{U}(t) \cdot y_{i}^{(I I, n)}(t)-y_{j}^{(I I I, n)}(t)-y_{j}^{(V, n)}(t)+y_{j}^{(V, n)}\left(t-\frac{1}{n}\right) \\
=\sum_{\left\{i: j \in N_{i}\right\}} b_{i j}^{L}(t) \cdot w_{l i}^{(* I, n)}+\sum_{\left\{i: j \in P_{i}\right\}} b_{i j}^{U}(t) \cdot w_{l i}^{(* I I, n)} \\
\quad+\max \left\{\mathfrak{f}^{(P N, n)}(t), \mathfrak{f}^{(N P, n)}(t)\right\} \cdot\left(\sum_{\left\{i: j \in N_{i}\right\}} b_{i j}^{L}(t)+\sum_{\left\{i: j \in P_{i}\right\}} b_{i j}^{U}(t)\right) \\
\quad-w_{l j}^{(* I I I, n)}-w_{l j}^{(* V, n)}+w_{l-1, j}^{(* V, n)}(\mathrm{by}(88)-(90) \text { and }(92)) \\
\geq \sum_{\left\{i: j \in N_{i}\right\}} b_{i j}^{L}(t) \cdot w_{l i}^{(* I, n)}+\sum_{\left\{i: j \in P_{i}\right\}} b_{i j}^{U}(t) \cdot w_{l i}^{(* I I, n)} \\
\quad+\mathfrak{f}^{(N P, n)}(t) \cdot\left(\sum_{\left\{i: j \in N_{i}\right\}} b_{i j}^{L}(t)+\sum_{\left\{i: j \in P_{i}\right\}} b_{i j}^{U}(t)\right)-w_{l j}^{(* I I, n)}-w_{l j}^{(* V, n)}+w_{l-1, j}^{(* V, n)}
\end{aligned}
$$

(by the nonnegativity from (16))

$$
\begin{aligned}
& =\sum_{\left\{i: j \in N_{i}\right\}} b_{l i j}^{(L, n)} \cdot w_{l i}^{(* I, n)}+\sum_{\left\{i: j \in P_{i}\right\}} b_{l i j}^{(U, n)} \cdot w_{l i}^{(* I I, n)} \\
& -w_{l j}^{(* I I, n)}-w_{l j}^{(* V, n)}+w_{l-1, j}^{(* V, n)}+g_{l j}^{(N P, n)}(t) \text { (by (93)) } \\
& \geq\left\{\begin{array}{ll}
a_{l j}^{(L, n)}+g_{l j}^{(N P, n)}(t) \text { for } j \in N & \text { (using the feasibility (51) for problem } \left.\left(\mathrm{D}_{n}\right)\right) \\
a_{l j}^{(U, n)}+g_{l j}^{(N P, n)}(t) \text { for } j \in P & \text { (using the feasibility (52) for problem } \left.\left(\mathrm{D}_{n}\right)\right)
\end{array}\right\} \\
& \geq\left\{\begin{array}{l}
a_{j}^{L}(t) \text { for } j \in N \\
a_{j}^{U}(t) \text { for } j \in P
\end{array}\right\} \quad(\text { by (94)). }
\end{aligned}
$$

- $\quad$ For $l=1$ and $t \in F_{1}^{(n)}$, we obtain

$$
\begin{gathered}
\sum_{\left\{i: j \in N_{i}\right\}} b_{i j}^{L}(t) \cdot y_{i}^{(I, n)}(t)+\sum_{\left\{i: j \in P_{i}\right\}} b_{i j}^{U}(t) \cdot y_{i}^{(I I, n)}(t)-y_{j}^{(I I I, n)}(t)-y_{j}^{(V, n)}(t) \\
=\sum_{\left\{i: j \in N_{i}\right\}} b_{i j}^{L}(t) \cdot w_{1 i}^{(* I, n)}+\sum_{\left\{i: j \in P_{i}\right\}} b_{i j}^{U}(t) \cdot w_{1 i}^{(* I I, n)} \\
+\max \left\{\mathfrak{f}^{(P N, n)}(t), \mathfrak{f}^{(N P, n)}(t)\right\} \cdot\left(\sum_{\left\{i: j \in N_{i}\right\}} b_{i j}^{L}(t)+\sum_{\left\{i: j \in P_{i}\right\}} b_{i j}^{U}(t)\right) \\
-w_{1 j}^{(* I I I, n)}-w_{1 j}^{(* V, n)}(\text { by }(88)-(90) \text { and }(92))
\end{gathered}
$$




$$
\begin{aligned}
& \geq \sum_{\left\{i: j \in N_{i}\right\}} b_{i j}^{L}(t) \cdot w_{1 i}^{(* I, n)}+\sum_{\left\{i: j \in P_{i}\right\}} b_{i j}^{U}(t) \cdot w_{1 i}^{(* I I, n)} \\
& \quad+\mathfrak{f}^{(N P, n)}(t) \cdot\left(\sum_{\left\{i: j \in N_{i}\right\}} b_{i j}^{L}(t)+\sum_{\left\{i: j \in P_{i}\right\}} b_{i j}^{U}(t)\right)-w_{1 j}^{(* I I, n)}-w_{1 j}^{(* V, n)} \\
& =\sum_{\left\{i: j \in N_{i}\right\}} b_{1 i j}^{(L, n)} \cdot w_{1 i}^{(* I, n)}+\sum_{\left\{i: j \in P_{i}\right\}} b_{1 i j}^{(U, n)} \cdot w_{1 i}^{(* I I, n)} \\
& \quad-w_{1 j}^{(* I I I, n)}-w_{1 j}^{(* V, n)}+g_{1 j}^{(U, n)}(t) \text { (by (93)) } \\
& \geq\left\{\begin{array}{c}
a_{1 j}^{(L, n)}+g_{1 j}^{(N P, n)}(t) \text { for } j \in N\left(\text { using the feasibility (47) for problem }\left(\mathrm{D}_{n}\right)\right) \\
a_{1 j}^{(U, n)}+g_{1 j}^{(N P, n)}(t) \text { for } j \in P\left(\text { using the feasibility (48) for problem }\left(\mathrm{D}_{n}\right)\right)
\end{array}\right\} \\
& \geq\left\{\begin{array}{l}
a_{j}^{L}(t) \text { for } j \in N \\
a_{j}^{U}(t) \text { for } j \in P
\end{array}\right\} \text { (by (94)). }
\end{aligned}
$$

- $\quad$ For $l=n$ and $t \in F_{n}^{(n)}$, i.e., $t-\frac{1}{n} \in F_{n-1}^{(n)}$, we obtain

$$
\begin{aligned}
& \sum_{\left\{i: j \in N_{i}\right\}} b_{i j}^{L}(t) \cdot y_{i}^{(I, n)}(t)+\sum_{\left\{i: j \in P_{i}\right\}} b_{i j}^{U}(t) \cdot y_{i}^{(I I, n)}(t)-y_{j}^{(I I I, n)}(t)+y_{j}^{(V, n)}\left(t-\frac{1}{n}\right) \\
& =\sum_{\left\{i: j \in N_{i}\right\}} b_{i j}^{L}(t) \cdot w_{n i}^{(* I, n)}+\sum_{\left\{i: j \in P_{i}\right\}} b_{i j}^{U}(t) \cdot w_{n i}^{(* I I, n)} \\
& +\max \left\{\mathfrak{f}^{(P N, n)}(t), \mathfrak{f}^{(N P, n)}(t)\right\} \cdot\left(\sum_{\left\{i: j \in N_{i}\right\}} b_{i j}^{L}(t)+\sum_{\left\{i: j \in P_{i}\right\}} b_{i j}^{U}(t)\right) \\
& -w_{n j}^{(* I I I, n)}+w_{n-1, j}^{(* V, n)}(\text { by }(88)-(90) \text { and (92)) } \\
& \geq \sum_{\left\{i: j \in N_{i}\right\}} b_{i j}^{L}(t) \cdot w_{n i}^{(* I, n)}+\sum_{\left\{i: j \in P_{i}\right\}} b_{i j}^{U}(t) \cdot w_{n i}^{(* I I, n)} \\
& +\mathfrak{f}^{(N P, n)}(t) \cdot\left(\sum_{\left\{i: j \in N_{i}\right\}} b_{i j}^{L}(t)+\sum_{\left\{i: j \in P_{i}\right\}} b_{i j}^{U}(t)\right)-w_{n j}^{(* I I I, n)}+w_{n-1, j}^{(* V, n)} \\
& =\sum_{\left\{i: j \in N_{i}\right\}} b_{n i j}^{(L, n)} \cdot w_{n i}^{(* I, n)}+\sum_{\left\{i: j \in P_{i}\right\}} b_{n i j}^{(U, n)} \cdot w_{n i}^{(* I I, n)} \\
& -w_{n j}^{(* I I I, n)}+w_{n-1, j}^{(* V, n)}+g_{n j}^{(U, n)}(t)(\text { by (93)) } \\
& \geq\left\{\begin{array}{ll}
a_{n j}^{(L, n)}+g_{n j}^{(N P, n)}(t) \text { for } j \in N & \text { (using the feasibility (55) for problem } \left.\left(\mathrm{D}_{n}\right)\right) \\
a_{n j}^{(U, n)}+g_{n j}^{(N P, n)}(t) \text { for } j \in P & \text { (using the feasibility (56) for problem } \left.\left(\mathrm{D}_{n}\right)\right)
\end{array}\right\}
\end{aligned}
$$




$$
\geq\left\{\begin{array}{l}
a_{j}^{L}(t) \text { for } j \in N \\
a_{j}^{U}(t) \text { for } j \in P
\end{array}\right\} \quad(\text { by }(94))
$$

- $\quad$ For $l=n$ and $t=1$, it is clear to see that $1-\frac{1}{n} \in F_{n}^{(n)}$. We obtain

$$
\begin{aligned}
& \sum_{\left\{i: j \in N_{i}\right\}} b_{i j}^{L}(1) \cdot y_{i}^{(I, n)}(1)+\sum_{\left\{i: j \in P_{i}\right\}} b_{i j}^{U}(1) \cdot y_{i}^{(I I, n)}(1)-y_{j}^{(I I I, n)}(1)+y_{j}^{(V, n)}\left(1-\frac{1}{n}\right) \\
& =\sum_{\left\{i: j \in N_{i}\right\}} b_{i j}^{L}(1) \cdot w_{n i}^{(* I, n)}+\sum_{\left\{i: j \in P_{i}\right\}} b_{i j}^{U}(1) \cdot w_{n i}^{(* I I, n)} \\
& +\max \left\{\mathfrak{f}^{(P N, n)}(1), \mathfrak{f}^{(N P, n)}(1)\right\} \cdot\left(\sum_{\left\{i: j \in N_{i}\right\}} b_{i j}^{L}(1)+\sum_{\left\{i: j \in P_{i}\right\}} b_{i j}^{U}(1)\right) \\
& -w_{n j}^{(* I I I, n)}+w_{n-1, j}^{(* V, n)} \text { (by (88)-(90) and (92)) } \\
& \geq \sum_{\left\{i: j \in N_{i}\right\}} b_{i j}^{L}(1) \cdot w_{n i}^{(* I, n)}+\sum_{\left\{i: j \in P_{i}\right\}} b_{i j}^{U}(1) \cdot w_{n i}^{(* I I, n)} \\
& +\mathfrak{f}^{(N P, n)}(1) \cdot\left(\sum_{\left\{i: j \in N_{i}\right\}} b_{i j}^{L}(1)+\sum_{\left\{i: j \in P_{i}\right\}} b_{i j}^{U}(1)\right)-w_{n j}^{(* I I, n)}+w_{n-1, j}^{(* V, n)} \\
& =\sum_{\left\{i: j \in N_{i}\right\}} b_{n i j}^{(L, n)} \cdot w_{n i}^{(* I, n)}+\sum_{\left\{i: j \in P_{i}\right\}} b_{n i j}^{(U, n)} \cdot w_{n i}^{(* I I, n)} \\
& -w_{n j}^{(* I I I, n)}+w_{n-1, j}^{(* V, n)}+g_{n j}^{(U, n)}(1)(\text { by (93)) } \\
& \geq\left\{\begin{array}{ll}
a_{n j}^{(L, n)}+g_{n j}^{(N P, n)}(1) \text { for } j \in N & \text { (using the feasibility (55) for problem } \left.\left(\mathrm{D}_{n}\right)\right) \\
a_{n j}^{(U, n)}+g_{n j}^{(N P, n)}(1) \text { for } j \in P & \text { (using the feasibility (56) for problem } \left.\left(\mathrm{D}_{n}\right)\right)
\end{array}\right\} \\
& \geq\left\{\begin{array}{l}
a_{j}^{L}(1) \text { for } j \in N \\
a_{j}^{U}(1) \text { for } j \in P
\end{array}\right\} \quad \text { (by (94)). }
\end{aligned}
$$

On the other hand, for $j=1, \cdots, q$, we define the real-valued functions $g_{l j}^{(L, n)}$ on $F_{l}^{(n)}$ for $l=1, \cdots, n-1$ and $g_{n j}^{(L, n)}$ on $\bar{E}_{n}^{(n)}$ by

$$
\begin{aligned}
g_{l j}^{(L, n)}(t)= & \sum_{\left\{i: j \in P_{i}\right\}}\left(b_{i j}^{L}(t)-b_{l i j}^{(L, n)}\right) \cdot w_{l i}^{(* I, n)}+\sum_{\left\{i: j \in N_{i}\right\}}\left(b_{i j}^{U}(t)-b_{l i j}^{(U, n)}\right) \cdot w_{l i}^{(* I I, n)} \\
& +\mathfrak{f}^{(P N, n)}(t) \cdot\left(\sum_{\left\{i: j \in P_{i}\right\}} b_{i j}^{L}(t)+\sum_{\left\{i: j \in N_{i}\right\}} b_{i j}^{U}(t)\right) .
\end{aligned}
$$


Using Lemma 3, we have

$$
\begin{aligned}
g_{l j}^{(P N, n)}(t) & =-h_{l j}^{(P N, n)}(t)+\mathfrak{f}^{(P N, n)}(t) \cdot\left(\sum_{\left\{i: j \in P_{i}\right\}} b_{i j}^{L}(t)+\sum_{\left\{i: j \in N_{i}\right\}} b_{i j}^{U}(t)\right) \\
& \geq\left\{\begin{array}{l}
a_{j}^{L}(t)-a_{l j}^{(L, n)} \text { for } j \in P \\
a_{j}^{U}(t)-a_{l j}^{(U, n)} \text { for } j \in N
\end{array}\right\} .
\end{aligned}
$$

We consider the following cases.

- $\quad$ For $l=2, \cdots, n-1$ and $t \in F_{l}^{(n)}$, i.e., $t-\frac{1}{n} \in F_{l-1}^{(n)}$, we obtain

$$
\begin{aligned}
& \sum_{\left\{i: j \in P_{i}\right\}} b_{i j}^{L}(t) \cdot y_{i}^{(I, n)}(t) \sum_{\left\{i: j \in N_{i}\right\}} b_{i j}^{U}(t) \cdot y_{i}^{(I I, n)}(t)+y_{j}^{(I I I, n)}(t)+y_{j}^{(I V, n)}(t)-y_{j}^{(I V, n)}\left(t-\frac{1}{n}\right) \\
& =\sum_{\left\{i: j \in P_{i}\right\}} b_{i j}^{L}(t) \cdot w_{l i}^{(* I, n)}+\sum_{\left\{i: j \in N_{i}\right\}} b_{i j}^{U}(t) \cdot w_{l i}^{(* I I, n)} \\
& \quad+\max \left\{\mathfrak{f}^{(P N, n)}(t), \mathfrak{f}^{(N P, n)}(t)\right\} \cdot\left(\sum_{\left\{i: j \in P_{i}\right\}} b_{i j}^{L}(t)+\sum_{\left\{i: j \in N_{i}\right\}} b_{i j}^{U}(t)\right) \\
& \quad+w_{l j}^{(* I I I, n)}+w_{l j}^{(* I V, n)}-w_{l-1, j}^{(* I V, n)}(\mathrm{by}(88)-(91)) \\
& \geq \sum_{\left\{i: j \in P_{i}\right\}} b_{i j}^{L}(t) \cdot w_{l i}^{(* I, n)}+\sum_{\left\{i: j \in N_{i}\right\}} b_{i j}^{U}(t) \cdot w_{l i}^{(* I I, n)} \\
& +\mathfrak{f}^{(P N, n)}(t) \cdot\left(\sum_{\left\{i: j \in P_{i}\right\}} b_{i j}^{L}(t)+\sum_{\left\{i: j \in N_{i}\right\}} b_{i j}^{U}(t)\right)+w_{l j}^{(* I I I, n)}+w_{l j}^{(* I V, n)}-w_{l-1, j}^{(* I V, n)}
\end{aligned}
$$

(by the nonnegativity from (15))

$$
\begin{aligned}
& =\sum_{\left\{i: j \in P_{i}\right\}} b_{l i j}^{(L, n)} \cdot w_{l i}^{(* I, n)}+\sum_{\left\{i: j \in N_{i}\right\}} b_{l i j}^{(U, n)} \cdot w_{l i}^{(* I I, n)} \\
& +w_{l j}^{(* I I I, n)}+w_{l j}^{(* I V, n)}-w_{l-1, j}^{(* I V, n)}+g_{l j}^{(P N, n)}(t)(\text { by }(95)) \\
& \geq\left\{\begin{array}{lc}
a_{l j}^{(L, n)}+g_{l j}^{(P N, n)}(t) \text { for } j \in P & \text { (using the feasibility (49) for problem } \left.\left(\mathrm{D}_{n}\right)\right) \\
a_{l j}^{(U, n)}+g_{l j}^{(P N, n)}(t) \text { for } j \in N & \text { (using the feasibility (50) for problem } \left.\left(\mathrm{D}_{n}\right)\right)
\end{array}\right\} \\
& \geq\left\{\begin{array}{l}
a_{j}^{L}(t) \text { for } j \in P \\
a_{j}^{U}(t) \text { for } j \in N
\end{array}\right\} \quad \text { (by (96)). }
\end{aligned}
$$

- $\quad$ For $l=1$ and $t \in F_{1}^{(n)}$, we obtain

$$
\sum_{\left\{i: j \in P_{i}\right\}} b_{i j}^{L}(t) \cdot y_{i}^{(I, n)}(t)+\sum_{\left\{i: j \in N_{i}\right\}} b_{i j}^{U}(t) \cdot y_{i}^{(I I, n)}(t)+y_{j}^{(I I I, n)}(t)+y_{j}^{(I V, n)}(t)
$$




$$
\begin{aligned}
& =\sum_{\left\{i: j \in P_{i}\right\}} b_{i j}^{L}(t) \cdot w_{1 i}^{(* I, n)}+\sum_{\left\{i: j \in N_{i}\right\}} b_{i j}^{U}(t) \cdot w_{1 i}^{(* I I, n)} \\
& +\max \left\{\mathfrak{f}^{(P N, n)}(t), \mathfrak{f}^{(N P, n)}(t)\right\} \cdot\left(\sum_{\left\{i: j \in P_{i}\right\}} b_{i j}^{L}(t)+\sum_{\left\{i: j \in N_{i}\right\}} b_{i j}^{U}(t)\right) \\
& +w_{1 j}^{(* I I I, n)}+w_{1 j}^{(* I V, n)}(\text { by }(88)-(91)) \\
& \geq \sum_{\left\{i: j \in P_{i}\right\}} b_{i j}^{L}(t) \cdot w_{1 i}^{(* I, n)}+\sum_{\left\{i: j \in N_{i}\right\}} b_{i j}^{U}(t) \cdot w_{1 i}^{(* I I, n)} \\
& +\mathfrak{f}^{(P N, n)}(t) \cdot\left(\sum_{\left\{i: j \in P_{i}\right\}} b_{i j}^{L}(t)+\sum_{\left\{i: j \in N_{i}\right\}} b_{i j}^{U}(t)\right)+w_{1 j}^{(* I I I, n)} \\
& =\sum_{\left\{i: j \in P_{i}\right\}} b_{1 i j}^{(L, n)} \cdot w_{1 i}^{(* I, n)}+\sum_{\left\{i: j \in N_{i}\right\}} b_{1 i j}^{(U, n)} \cdot w_{1 i}^{(* I I, n)} \\
& +w_{1 j}^{(* I I I, n)}+w_{1 j}^{(* I V, n)}+g_{1 j}^{(L, n)}(t)(\text { by (95)) } \\
& \geq\left\{\begin{array}{l}
\left.a_{1 j}^{(L, n)}+g_{1 j}^{(P N, n)}(t) \text { for } j \in P \text { (using the feasibility (45) for problem }\left(\mathrm{D}_{n}\right)\right) \\
\left.a_{1 j}^{(U, n)}+g_{1 j}^{(P N, n)}(t) \text { for } j \in N \text { (using the feasibility (46) for problem }\left(\mathrm{D}_{n}\right)\right)
\end{array}\right\} \\
& \geq\left\{\begin{array}{l}
a_{j}^{L}(t) \text { for } j \in P \\
a_{j}^{U}(t) \text { for } j \in N
\end{array}\right\} \text { (by (96)). }
\end{aligned}
$$

- $\quad$ For $l=n$ and $t \in F_{n}^{(n)}$, i.e., $t-\frac{1}{n} \in F_{n-1}^{(n)}$, we obtain

$$
\begin{aligned}
& \sum_{\left\{i: j \in P_{i}\right\}} b_{i j}^{L}(t) \cdot y_{i}^{(I, n)}(t)+\sum_{\left\{i: j \in N_{i}\right\}} b_{i j}^{U}(t) \cdot y_{i}^{(I I, n)}(t)+y_{j}^{(I I I, n)}(t)-y_{j}^{(I V, n)}\left(t-\frac{1}{n}\right) \\
& =\sum_{\left\{i: j \in P_{i}\right\}} b_{i j}^{L}(t) \cdot w_{n i}^{(* I, n)}+\sum_{\left\{i: j \in N_{i}\right\}} b_{i j}^{L}(t) \cdot w_{n i}^{(* I I, n)} \\
& +\max \left\{\mathfrak{f}^{(P N, n)}(t), \mathfrak{f}^{(N P, n)}(t)\right\} \cdot\left(\sum_{\left\{i: j \in P_{i}\right\}} b_{i j}^{L}(t)+\sum_{\left\{i: j \in N_{i}\right\}} b_{i j}^{U}(t)\right) \\
& +w_{n j}^{(* I I I, n)}-w_{n-1, j}^{(* I V, n)}(\text { by }(88)-(91)) \\
& \geq \sum_{\left\{i: j \in P_{i}\right\}} b_{i j}^{L}(t) \cdot w_{n i}^{(* I, n)}+\sum_{\left\{i: j \in N_{i}\right\}} b_{i j}^{L}(t) \cdot w_{n i}^{(* I I, n)} \\
& +\mathfrak{f}^{(P N, n)}(t) \cdot\left(\sum_{\left\{i: j \in P_{i}\right\}} b_{i j}^{L}(t)+\sum_{\left\{i: j \in N_{i}\right\}} b_{i j}^{U}(t)\right)+w_{n j}^{(* I I, n)}-w_{n-1, j}^{(* I V, n)}
\end{aligned}
$$




$$
\begin{aligned}
& =\sum_{\left\{i: j \in P_{i}\right\}} b_{n i j}^{(L, n)} \cdot w_{n i}^{(* I, n)} \sum_{\left\{i: j \in N_{i}\right\}} b_{n i j}^{(U, n)} \cdot w_{n i}^{(* I I, n)} \\
& +w_{n j}^{(* I I I, n)}-w_{n-1, j}^{(* I V, n)}+g_{n j}^{(L, n)}(t)(\text { by (95)) } \\
& \geq\left\{\begin{array}{ll}
a_{n j}^{(L, n)}+g_{n j}^{(P N, n)}(t) \text { for } j \in P & \text { (using the feasibility (53) for problem } \left.\left(\mathrm{D}_{n}\right)\right) \\
a_{n j}^{(U, n)}+g_{n j}^{(P N, n)}(t) \text { for } j \in N & \text { (using the feasibility (54) for problem } \left.\left(\mathrm{D}_{n}\right)\right)
\end{array}\right\} \\
& \geq\left\{\begin{array}{l}
a_{j}^{L}(t) \text { for } j \in P \\
a_{j}^{U}(t) \text { for } j \in N
\end{array}\right\} \quad(\text { by (96)). }
\end{aligned}
$$

- For $l=n$ and $t=1$, it is clear to see that $1-\frac{1}{n} \in F_{n}^{(n)}$. We obtain

$$
\begin{aligned}
& \sum_{\left\{i: j \in P_{i}\right\}} b_{i j}^{L}(1) \cdot y_{i}^{(I, n)}(1) \sum_{\left\{i: j \in N_{i}\right\}} b_{i j}^{U}(1) \cdot y_{i}^{(I I, n)}(1)+y_{j}^{(I I I, n)}(1)-y_{j}^{(I V, n)}\left(1-\frac{1}{n}\right) \\
& =\sum_{\left\{i: j \in P_{i}\right\}} b_{i j}^{L}(1) \cdot w_{n i}^{(* I, n)}+\sum_{\left\{i: j \in N_{i}\right\}} b_{i j}^{U}(1) \cdot w_{n i}^{(* I I, n)} \\
& +\max \left\{\mathfrak{f}^{(P N, n)}(1), \mathfrak{f}^{(N P, n)}(1)\right\} \cdot\left(\sum_{\left\{i: j \in P_{i}\right\}} b_{i j}^{L}(1)+\sum_{\left\{i: j \in N_{i}\right\}} b_{i j}^{U}(1)\right) \\
& +w_{n j}^{(* I I I, n)}-w_{n-1, j}^{(* I V, n)}(\text { by }(88)-(91)) \\
& \geq \sum_{\left\{i: j \in P_{i}\right\}} b_{i j}^{L}(1) \cdot w_{n i}^{(* I, n)}+\sum_{\left\{i: j \in N_{i}\right\}} b_{i j}^{U}(1) \cdot w_{n i}^{(* I I, n)} \\
& +\mathfrak{f}^{(P N, n)}(1) \cdot\left(\sum_{\left\{i: j \in P_{i}\right\}} b_{i j}^{L}(1)+\sum_{\left\{i: j \in N_{i}\right\}} b_{i j}^{U}(1)\right)+w_{n j}^{(* I I I, n)}-w_{n-1, j}^{(* I V, n)} \\
& =\sum_{\left\{i: j \in P_{i}\right\}} b_{n i j}^{(L, n)} \cdot w_{n i}^{(* I, n)} \sum_{\left\{i: j \in N_{i}\right\}} b_{n i j}^{(U, n)} \cdot w_{n i}^{(* I I, n)} \\
& +w_{n j}^{(* I I I, n)}-w_{n-1, j}^{(* I V, n)}+g_{n j}^{(L, n)}(1)(\text { by }(95)) \\
& \geq\left\{\begin{array}{cc}
a_{n j}^{(L, n)}+g_{n j}^{(P N, n)}(1) \text { for } j \in P & \text { (using the feasibility (53) for problem } \left.\left(\mathrm{D}_{n}\right)\right) \\
a_{n j}^{(U, n)}+g_{n j}^{(P N, n)}(1) \text { for } j \in N & \text { (using the feasibility (54) for problem } \left.\left(\mathrm{D}_{n}\right)\right)
\end{array}\right\} \\
& \geq\left\{\begin{array}{l}
a_{j}^{L}(1) \text { for } j \in P \\
a_{j}^{U}(1) \text { for } j \in N
\end{array}\right\} \quad(\text { by (96)) }
\end{aligned}
$$

This completes the proof. 
For $i=1, \cdots, p$ and $j=1, \cdots, q$, we define the real-valued functions:

$$
\begin{gathered}
\bar{c}_{i}^{(L, n)}(t)= \begin{cases}c_{l i}^{(L, n)} & \text { if } t \in F_{l}^{(n)} \text { for } l=1, \cdots, n \\
c_{n i}^{(L, n)} & \text { if } t=1\end{cases} \\
\bar{c}_{i}^{(U, n)}(t)= \begin{cases}c_{l i}^{(U, n)} & \text { if } t \in F_{l}^{(n)} \text { for } l=1, \cdots, n \\
c_{n i}^{(U, n)} & \text { if } t=1\end{cases} \\
\bar{w}_{i}^{(* I, n)}(t)= \begin{cases}w_{l i}^{(* I, n)} & \text { if } t \in F_{l}^{(n)} \text { for } l=1, \cdots, n \\
w_{n i}^{(* I, n)} & \text { if } t=1\end{cases} \\
\bar{w}_{i}^{(* I I, n)}(t)= \begin{cases}w_{l i}^{(* I I, n)} & \text { if } t \in F_{l}^{(n)} \text { for } l=1, \cdots, n \\
w_{n i}^{(* I, n)} & \text { if } t=1\end{cases}
\end{gathered}
$$

Lemma 4. For $i=1, \cdots, p$ and $j=1, \cdots, q$, we have

$$
\int_{0}^{1}\left[c_{i}^{L}(t)-\bar{c}_{i}^{(L, n)}(t)\right] \cdot \bar{w}_{i}^{(* I, n)}(t) d t \rightarrow 0 \text { as } n \rightarrow \infty,
$$

and

$$
\int_{0}^{1}\left[c_{i}^{U}(t)-\bar{c}_{i}^{(U, n)}(t)\right] \cdot \bar{w}_{i}^{(* I I, n)}(t) d t \rightarrow 0 \text { as } n \rightarrow \infty .
$$

Proof. It is clear to see that the following functions

$$
\left[c_{i}^{L}(t)-\bar{c}_{i}^{(L, n)}(t)\right] \cdot \bar{w}_{i}^{(* I, n)}(t) \text { and }\left[c_{i}^{U}(t)-\bar{c}_{i}^{(U, n)}(t)\right] \cdot \bar{w}_{i}^{(* I I, n)}(t)
$$

are continuous a.e. on $[0,1]$, i.e., they are Riemann-integrable on $[0,1]$. In other words, their Riemann integral and Lebesgue integral are identical. From Lemma 1 , for $i=1, \cdots, p, l=1, \cdots, n$ and $t \in F_{l}^{(n)}$, we see that

$$
0 \leq c_{i}^{L}(t)-\bar{c}_{i}^{(L, n)}(t)=c_{i}^{L}(t)-c_{l i}^{(L, n)} \leq \sup _{t \in F_{l}^{(n)}}\left[c_{i}^{L}(t)-c_{l i}^{(L, n)}\right] \rightarrow 0 \text { as } n \rightarrow \infty
$$

and

$$
0 \leq c_{i}^{U}(t)-\bar{c}_{i}^{(U, n)}(t)=c_{i}^{U}(t)-c_{l i}^{(U, n)} \leq \sup _{t \in F_{l}^{(n)}}\left[c_{i}^{U}(t)-c_{l i}^{(U, n)}\right] \rightarrow 0 \text { as } n \rightarrow \infty .
$$

Therefore we obtain

$$
c_{i}^{L}(t)-\bar{c}_{i}^{(L, n)}(t) \rightarrow 0 \text { and } c_{i}^{U}(t)-\bar{c}_{i}^{(U, n)}(t) \rightarrow 0 \text { as } n \rightarrow \infty \text { a.e. on }[0,1] .
$$

Since the sequences $\left\{\bar{w}_{i}^{(* I, n)}\right\}_{n=1}^{\infty}$ and $\left\{\bar{w}_{i}^{(* I I, n)}\right\}_{n=1}^{\infty}$ are uniformly bounded by part (ii) of Proposition 5, using (104), (103) and the Lebesgue bounded convergence theorem for integrals by referring to Royden [34], we can obtain (101) and (102). This completes the proof.

Theorem 3. Given an optimal solution $\mathbf{z}^{(* n)}$ of primal problem $\left(\mathrm{P}_{n}\right)$ and an optimal solution $\mathbf{w}^{(* n)}$ of dual problem $\left(\mathrm{D}_{n}\right)$, we define the vector-valued functions

$$
\mathbf{x}^{(n)}=\left(\mathbf{x}^{(L, n)}, \mathbf{x}^{(U, n)}\right) \text { and } \mathbf{y}^{(n)}=\left(\mathbf{y}^{(I, n)}, \mathbf{y}^{(I I, n)}, \mathbf{y}^{(I I I, n)}, \mathbf{y}^{(I V, n)}, \mathbf{y}^{(V, n)}\right)
$$

according to (62) and (63) and (88)-(92), respectively. Then the following statements hold true. 
(i) We have

$$
\limsup _{n \rightarrow \infty} V\left(\operatorname{DSOP}_{n}\right)=\underset{n \rightarrow \infty}{\limsup } V\left(\mathrm{D}_{n}\right)
$$

and

$$
0 \leq V\left(\mathrm{DSOP}_{n}\right)-V\left(\mathrm{D}_{n}\right) \leq \varepsilon_{n}
$$

where

$$
\begin{aligned}
\varepsilon_{n}= & -V\left(\mathrm{D}_{n}\right)+\sum_{l=1}^{n} \sum_{i=1}^{p}\left[\left(w_{l i}^{(* I, n)}+\max \left\{\frac{\pi_{l}^{(P N, n)}}{\left.\left.\left.\mathfrak{b}_{l}^{(P N, n)}, \frac{\pi_{l}^{(N P, n)}}{\mathfrak{b}_{l}^{(N P, n)}}\right\}\right) \cdot \int_{\bar{E}_{l}^{(n)}} c_{i}^{L}(t) d t\right]}\right.\right.\right. \\
& +\sum_{l=1}^{n} \sum_{i=1}^{p}\left[\left(w_{l i}^{(* I I, n)}+\max \left\{\frac{\pi_{l}^{(P N, n)}}{\mathfrak{b}_{l}^{(P N, n)},} \frac{\pi_{l}^{(N P, n)}}{\mathfrak{b}_{l}^{(N P, n)}}\right\}\right) \cdot \int_{\bar{E}_{l}^{(n)}} c_{i}^{U}(t) d t\right]
\end{aligned}
$$

satisfying $\varepsilon_{n} \rightarrow 0$ as $n \rightarrow \infty$. Moreover, there exist two convergent subsequences $\left\{V\left(\mathrm{DSOP}_{n_{k}}\right)\right\}_{k=1}^{\infty}$ and $\left\{V\left(\mathrm{D}_{n_{k}}\right)\right\}_{k=1}^{\infty}$ of $\left\{V\left(\mathrm{DSOP}_{n}\right)\right\}_{n=1}^{\infty}$ and $\left\{V\left(\mathrm{D}_{n}\right)\right\}_{n=1}^{\infty}$, respectively, such that

$$
\lim _{k \rightarrow \infty} V\left(\mathrm{DSOP}_{n_{k}}\right)=\lim _{k \rightarrow \infty} V\left(\mathrm{D}_{n_{k}}\right)
$$

(ii) (Asymptotic No Duality Gap). We have

$$
V(\mathrm{SOP})=\limsup _{n \rightarrow \infty} V\left(\mathrm{DSOP}_{n}\right)=\limsup _{n \rightarrow \infty} V\left(\mathrm{SOP}_{n}\right)=\limsup _{n \rightarrow \infty} V\left(\mathrm{D}_{n}\right)=\limsup _{n \rightarrow \infty} V\left(\mathrm{P}_{n}\right)
$$

and

$$
V(\mathrm{SOP})=\lim _{k \rightarrow \infty} V\left(\mathrm{DSOP}_{n_{k}}\right)=\lim _{k \rightarrow \infty} V\left(\mathrm{SOP}_{n_{k}}\right)=\lim _{k \rightarrow \infty} V\left(\mathrm{D}_{n_{k}}\right)=\lim _{k \rightarrow \infty} V\left(\mathrm{P}_{n_{k}}\right)
$$

and

$$
0 \leq V(\mathrm{SOP})-V\left(\mathrm{P}_{n}\right) \leq \varepsilon_{n} .
$$

Proof. To prove part (i), we have

$$
\begin{aligned}
& 0 \leq V\left(\operatorname{DSOP}_{n}\right)-V\left(\mathrm{D}_{n}\right)(\text { by (69)) } \\
& =V\left(\operatorname{DSOP}_{n}\right)-\sum_{i=1}^{p} \sum_{l=1}^{n} \frac{1}{n} \cdot c_{l i}^{(L, n)} \cdot w_{l i}^{(* I, n)}-\sum_{i=1}^{p} \sum_{l=1}^{n} \frac{1}{n} \cdot c_{l i}^{(U, n)} \cdot w_{l i}^{(* I, n)} \\
& =V\left(\mathrm{DSOP}_{n}\right)-\sum_{l=1}^{n} \sum_{i=1}^{p} \int_{\bar{E}_{l}^{(n)}} c_{l i}^{(L, n)} \cdot w_{l i}^{(* I, n)} d t-\sum_{l=1}^{n} \sum_{i=1}^{p} \int_{\bar{E}_{l}^{(n)}} c_{l i}^{(U, n)} \cdot w_{l i}^{(* I I, n)} \\
& \leq \sum_{i=1}^{p} \int_{0}^{1} c_{i}^{L}(t) \cdot y_{i}^{(I, n)}(t) d t+\sum_{i=1}^{p} \int_{0}^{1} c_{i}^{U}(t) \cdot y_{i}^{(I I, n)}(t) d t \\
& -\sum_{l=1}^{n} \sum_{i=1}^{p} \int_{\bar{E}_{l}^{(n)}} c_{l i}^{(L, n)} \cdot w_{l i}^{(* I, n)} d t-\sum_{l=1}^{n} \sum_{i=1}^{p} \int_{\bar{E}_{l}^{(n)}} c_{l i}^{(U, n)} \cdot w_{l i}^{(* I I, n)} \text { (by Proposition 7) } \\
& =\sum_{i=1}^{p} \sum_{l=1}^{n} \int_{\bar{E}_{l}^{(n)}}\left(c_{i}^{L}(t)-c_{l i}^{(L, n)}\right) \cdot w_{l i}^{(* I, n)} d t+\sum_{i=1}^{p} \int_{0}^{1} \max \left\{\mathfrak{f}^{(P N, n)}(t), \mathfrak{f}^{(N P, n)}(t)\right\} \cdot c_{i}^{L}(t) d t \\
& +\sum_{i=1}^{p} \sum_{l=1}^{n} \int_{\bar{E}_{l}^{(n)}}\left(c_{i}^{U}(t)-c_{l i}^{(U, n)}\right) \cdot w_{l i}^{(* I I, n)} d t+\sum_{i=1}^{p} \int_{0}^{1} \max \left\{\mathfrak{f}^{(P N, n)}(t), \mathfrak{f}^{(N P, n)}(t)\right\} \cdot c_{i}^{U}(t) d t \\
& \text { (using (88) and (89)) } \\
& \equiv \varepsilon_{n} \text {, }
\end{aligned}
$$

which implies

$$
V\left(\mathrm{D}_{n}\right) \leq V\left(\mathrm{DSOP}_{n}\right) \leq V\left(\mathrm{D}_{n}\right)+\varepsilon_{n} .
$$


Now we have

$$
\begin{aligned}
0 & \leq \sum_{l=1}^{n} \int_{\bar{E}_{l}^{(n)}}\left(c_{i}^{L}(t)-c_{l i}^{(L, n)}\right) \cdot w_{l i}^{(* I, n)} d t=\sum_{l=1}^{n} \int_{F_{l}^{(n)}}\left(c_{i}^{L}(t)-c_{l i}^{(L, n)}\right) \cdot w_{l i}^{(* I, n)} d t \\
& =\int_{0}^{1}\left[c_{i}^{L}(t)-\bar{c}_{i}^{(L, n)}(t)\right] \cdot \bar{w}_{i}^{(* I, n)}(t) d t \rightarrow 0 \text { as } n \rightarrow \infty(\operatorname{using}(97),(99) \text { and (101)) }
\end{aligned}
$$

and

$$
0 \leq \sum_{l=1}^{n} \int_{\bar{E}_{l}^{(n)}}\left(c_{i}^{U}(t)-c_{l i}^{(U, n)}\right) \cdot w_{l i}^{(* I I, n)} d t \rightarrow 0 \text { as } n \rightarrow \infty
$$

by using (98), (100) and (102).

By applying Lemma 2 to (84) and (85), it follows that $\mathfrak{p}^{(P N, n)} \rightarrow 0$ and $\mathfrak{p}^{(N P, n)} \rightarrow 0$ as $n \rightarrow \infty$ a.e. on $[0,1]$. By applying (83) to (87), we see that $\mathfrak{f}^{(P N, n)} \rightarrow 0$ and $\mathfrak{f}^{(N P, n)} \rightarrow 0$ as $n \rightarrow \infty$ a.e. on $[0,1]$. Using the Lebesgue bounded convergence theorem for integrals (ref. Royden [34]), we obtain

$$
0 \leq \int_{0}^{1} \max \left\{\mathfrak{f}^{(P N, n)}(t), \mathfrak{f}^{(N P, n)}(t)\right\} \cdot c_{i}^{L}(t) d t \rightarrow 0 \text { as } n \rightarrow \infty
$$

and

$$
0 \leq \int_{0}^{1} \max \left\{\mathfrak{f}^{(P N, n)}(t), \mathfrak{f}^{(N P, n)}(t)\right\} \cdot c_{i}^{U}(t) d t \rightarrow 0 \text { as } n \rightarrow \infty .
$$

Therefore we conclude that $\varepsilon_{n} \rightarrow 0$ as $n \rightarrow \infty$. From (109), we obtain

$$
\begin{aligned}
\limsup _{n \rightarrow \infty} V\left(\mathrm{D}_{n}\right) & \leq \limsup _{n \rightarrow \infty} V\left(\mathrm{DSOP}_{n}\right) \leq \underset{n \rightarrow \infty}{\limsup }\left(V\left(\mathrm{D}_{n}\right)+\varepsilon_{n}\right) \\
& \leq \limsup _{n \rightarrow \infty} V\left(\mathrm{D}_{n}\right)+\underset{n \rightarrow \infty}{\limsup \varepsilon_{n}=} \limsup _{n \rightarrow \infty} V\left(\mathrm{D}_{n}\right),
\end{aligned}
$$

which proves (105). From the inequality (38), we see that $\left\{V\left(\operatorname{DSOP}_{n}\right)\right\}_{n=1}^{\infty}$ is a bounded sequence, which says that there exists a convergent subsequence $\left\{V\left(\operatorname{DSOP}_{n_{k}}\right)\right\}_{k=1}^{\infty}$ of $\left\{V\left(\operatorname{DSOP}_{n}\right)\right\}_{n=1}^{\infty}$. From the inequality (60), we also see that $\left\{V\left(D_{n_{k}}\right)\right\}_{k=1}^{\infty}$ is a bounded sequence, which also says that there exists a convergent subsequence $\left\{V\left(\mathrm{D}_{n_{k_{r}}}\right)\right\}_{r=1}^{\infty}$ of $\left\{V\left(\mathrm{D}_{n_{k}}\right)\right\}_{k=1}^{\infty}$. In other words, the two subsequences $\left\{V\left(\mathrm{DSOP}_{n_{k_{r}}}\right)\right\}_{r=1}^{\infty}$ and $\left\{V\left(\mathrm{D}_{n_{k_{r}}}\right)\right\}_{r=1}^{\infty}$ are convergent. Using (109) again, we obtain

$$
\lim _{r \rightarrow \infty} V\left(\mathrm{DSOP}_{n_{k_{r}}}\right)=\lim _{r \rightarrow \infty} V\left(\mathrm{D}_{n_{k_{r}}}\right),
$$

which is equivalent to (107).

Moreover the error $\varepsilon_{n}$ can be rewritten as

$$
\begin{aligned}
\varepsilon_{n}= & -V\left(\mathrm{D}_{n}\right)+\sum_{l=1}^{n} \sum_{i=1}^{p} \int_{\bar{E}_{l}^{(n)}} c_{i}^{L}(t) \cdot w_{l i}^{(* I, n)} d t+\sum_{l=1}^{n} \sum_{i=1}^{p} \int_{\bar{E}_{l}^{(n)}} c_{i}^{U}(t) \cdot w_{l i}^{(* I I, n)} d t \\
& +\sum_{l=1}^{n} \sum_{i=1}^{p} \max \left\{\frac{\pi_{l}^{(P N, n)}}{\mathfrak{b}_{l}^{(P N, n)}}, \frac{\pi_{l}^{(N P, n)}}{\mathfrak{b}_{l}^{(N P, n)}}\right\} \cdot\left[\int_{\bar{E}_{l}^{(n)}} c_{i}^{L}(t) d t+\int_{\bar{E}_{l}^{(n)}} c_{i}^{U}(t) d t\right],
\end{aligned}
$$

which implies the expression (106). This proves part (i).

To prove part (ii), since $V\left(\mathrm{D}_{n}\right)=V\left(\mathrm{P}_{n}\right)$ for each $n \in \mathbb{N}$, using part (i) and inequality (69), we obtain

$$
0 \leq V(\mathrm{SOP})-V\left(\mathrm{P}_{n}\right) \leq V\left(\mathrm{DSOP}_{n}\right)-V\left(\mathrm{D}_{n}\right) \leq \varepsilon_{n}
$$

and

$$
\limsup _{n \rightarrow \infty} V\left(\operatorname{DSOP}_{n}\right) \geq \underset{n \rightarrow \infty}{\limsup } V\left(\mathrm{SOP}_{n}\right) \geq V(\mathrm{SOP}) \geq \limsup _{n \rightarrow \infty} V\left(\mathrm{D}_{n}\right)=\limsup _{n \rightarrow \infty} V\left(\mathrm{DSOP}_{n}\right) .
$$


Using $V\left(\mathrm{D}_{n}\right)=V\left(\mathrm{P}_{n}\right)$, we also have

$$
\limsup _{n \rightarrow \infty} V\left(\mathrm{DSOP}_{n}\right)=\limsup _{n \rightarrow \infty} V\left(\mathrm{SOP}_{n}\right)=V(\mathrm{SOP})=\limsup _{n \rightarrow \infty} V\left(\mathrm{D}_{n}\right)=\limsup _{n \rightarrow \infty} V\left(\mathrm{P}_{n}\right) .
$$

This completes the proof.

The following result presents the error estimation by simply solving the conventional linear programming problem $\left(\mathrm{P}_{n}\right)$.

Theorem 4. Given an optimal solution $\mathbf{z}^{(* n)}$ of problem $\left(\mathrm{P}_{n}\right)$, let $\mathbf{x}^{(n)}=\left(\mathbf{x}^{(L, n)}, \mathbf{x}^{(U, n)}\right)$ be defined in (62) and (63). Then the error between $V(\mathrm{SOP})$ and the objective value of $\mathbf{x}^{(n)}$ is less than or equal to $\varepsilon_{n}$ defined in (106). In other words, we have

$$
0 \leq V(\mathrm{SOP})-\left(\sum_{j=1}^{q} \int_{0}^{1} a_{j}^{L}(t) \cdot x_{j}^{(L, n)}(t) d t+\sum_{j=1}^{q} \int_{0}^{1} a_{j}^{U}(t) \cdot x_{j}^{(U, n)}(t) d t\right) \leq \varepsilon_{n},
$$

where $\varepsilon_{n} \rightarrow 0$ as $n \rightarrow \infty$.

Proof. Proposition 6 says that $\mathbf{x}^{(n)}$ is a feasible solution of problem SOP with the objective value given by

$$
\begin{aligned}
& \sum_{j=1}^{q} \int_{0}^{1} a_{j}^{L}(t) \cdot x_{j}^{(L, n)}(t) d t+\sum_{j=1}^{q} \int_{0}^{1} a_{j}^{U}(t) \cdot x_{j}^{(U, n)}(t) d t \\
& \quad=\sum_{j=1}^{q} \sum_{l=1}^{n} \int_{\bar{E}_{l}^{(n)}} a_{j}^{L}(t) \cdot x_{j}^{(L, n)}(t) d t+\sum_{j=1}^{q} \sum_{l=1}^{n} \int_{\bar{E}_{l}^{(n)}} a_{j}^{U}(t) \cdot x_{j}^{(U, n)}(t) d t \\
& \quad \geq \sum_{j=1}^{q} \sum_{l=1}^{n} \int_{\bar{E}_{l}^{(n)}} a_{l j}^{(L, n)} \cdot z_{l j}^{(* L, n)} d t+\sum_{j=1}^{q} \sum_{l=1}^{n} \int_{\bar{E}_{l}^{(n)}} a_{l j}^{(U, n)} \cdot z_{l j}^{(* U, n)} d t(\text { by (39)) } \\
& \quad=\sum_{j=1}^{q} \sum_{l=1}^{n} \frac{1}{n} \cdot a_{l j}^{(L, n)} \cdot z_{l j}^{(* L, n)}+\sum_{j=1}^{q} \sum_{l=1}^{n} \frac{1}{n} \cdot a_{l j}^{(U, n)}(t) \cdot z_{l j}^{(* U, n)} \\
& \quad=V\left(\mathrm{P}_{n}\right)=V\left(\mathrm{D}_{n}\right) .
\end{aligned}
$$

It follows that

$$
\begin{aligned}
0 & \leq V(\mathrm{SOP})-\left(\sum_{j=1}^{q} \int_{0}^{1} a_{j}^{L}(t) \cdot x_{j}^{(L, n)}(t) d t+\sum_{j=1}^{q} \int_{0}^{1} a_{j}^{U}(t) \cdot x_{j}^{(U, n)}(t) d t\right) \\
& \leq V\left(\mathrm{DSOP}_{n}\right)-V\left(\mathrm{D}_{n}\right)(\text { by }(112) \text { and (69)) } \\
& \leq \varepsilon_{n}(\text { by (108)). }
\end{aligned}
$$

This completes the proof.

Recall that $V$ (SOP) denotes the supremum of the objective function of problem SOP. However, the supremum $V(\mathrm{SOP})$ may not be attained. In other words, there may not exist a feasible solution $\mathbf{x}^{*}$ such that its objective value is equal to the supremum $V(\mathrm{SOP})$. In the next section, we shall provide the sufficient conditions to guarantee the existence of optimal solutions. From the computational viewpoint, it suffices to consider the concept of so-called $\epsilon$-optimal solution that is formally defined below. 
Definition 2. Given any sufficiently small $\epsilon>0$, we say that the feasible solution $\mathbf{x}^{(\epsilon)}=\left(\mathbf{x}^{(L, \epsilon)}, \mathbf{x}^{(U, \epsilon)}\right)$ of problem SOP is an $\epsilon$-optimal solution if and only if the difference between $V(S O P)$ and the objective value of $\mathbf{x}^{(\epsilon)}$ is less than $\epsilon$. More precisely, the feasible solution $\mathbf{x}^{(\epsilon)}$ is an $\epsilon$-optimal solution if and only if

$$
0 \leq V(S O P)-\left(\sum_{j=1}^{q} \int_{0}^{1} a_{j}^{L}(t) \cdot x_{j}^{(L, \epsilon)}(t) d t+\sum_{j=1}^{q} \int_{0}^{1} a_{j}^{U}(t) \cdot x_{j}^{(U, \epsilon)}(t) d t\right)<\epsilon .
$$

Now we have the following interesting result.

Theorem 5. Given any sufficiently small $\epsilon>0$, the $\epsilon$-optimal solution of problem SOP always exists in the following sense: there exists $n \in \mathbb{N}$ such that we can take $\mathbf{x}^{(\epsilon)}=\mathbf{x}^{(n)}$ that is obtained from Theorem 4 satisfying $\varepsilon_{n}<\epsilon$.

Proof. Theorem 4 says that $\varepsilon_{n} \rightarrow 0$ as $n \rightarrow \infty$. Therefore, given any sufficiently small $\epsilon>0$, there exists a sufficiently large $n \in \mathbb{N}$ such that $\varepsilon_{n}<\epsilon$. Then the result follows immediately.

\section{Existence of Optimal Solution}

We shall present the convergent properties of the sequences $\left\{\mathbf{x}^{(n)}\right\}_{n=1}^{\infty}$ and $\left\{\mathbf{y}^{(n)}\right\}_{n=1}^{\infty}$ that are constructed from the optimal solutions $\mathbf{z}^{(* n)}$ of primal problem $\left(\mathrm{P}_{n}\right)$ and the optimal solution $\mathbf{w}^{(* n)}$ of dual problem $\left(\mathrm{D}_{n}\right)$ according to (62) and (63) and (88)-(92), respectively. In this case, we shall provide the sufficient conditions to guarantee the existence of optimal solutions of problem SOP.

Theorem 6. Given an optimal solution $\mathbf{z}^{(* n)}$ of primal problem $\left(\mathrm{P}_{n}\right)$ and an optimal solution $\mathbf{w}^{(* n)}$ of dual problem $\left(\mathrm{D}_{n}\right)$, we define

$$
\mathbf{x}^{(n)}=\left(\mathbf{x}^{(L, n)}, \mathbf{x}^{(U, n)}\right) \text { and } \mathbf{y}^{(n)}(t)=\left(\mathbf{y}^{(I, n)}(t), \mathbf{y}^{(I I, n)}(t), \mathbf{y}^{(I I I, n)}(t), \mathbf{y}^{(I V, n)}(t), \mathbf{y}^{(V, n)}(t)\right)
$$

according to (62) and (63) and (88)-(92), respectively. Considering the component functions $x_{j}^{(L, n)}$ and $x_{j}^{(U, n)}$ of $\mathbf{x}^{(L, n)}$ and $\mathbf{x}^{(U, n)}$, respectively, for $j=1, \cdots, q$, we define the real-valued functions $x_{j}^{(L, 0)}$ and $x_{j}^{(U, 0)}$ on $[0,1]$ by

$$
x_{j}^{(L, 0)}(t)=\liminf _{n \rightarrow \infty} x_{j}^{(L, n)}(t) \text { and } x_{j}^{(U, 0)}(t)=\liminf _{n \rightarrow \infty} x_{j}^{(U, n)}(t),
$$

which can form the vector-valued functions $\mathbf{x}^{(0)}=\left(\mathbf{x}^{(L, 0)}, \mathbf{x}^{(U, 0)}\right)$ on $[0,1]$. Suppose that the objective value of $\mathbf{x}^{(0)}$ with respect to the primal problem $\left(\mathrm{P}_{n}\right)$ is greater than or equal to the objective value of $\mathbf{y}^{(n)}$ with respect to the dual problem $\left(\mathrm{D}_{n}\right)$; that is,

$$
\begin{aligned}
& \sum_{j \in P}\left(\int_{0}^{1} a_{j}^{L}(t) \cdot x_{j}^{(L, 0)}(t) d \alpha+\int_{0}^{1} a_{j}^{U}(t) \cdot x_{j}^{(U, 0)}(t) d \alpha\right) \\
& \quad+\sum_{j \in N}\left(\int_{0}^{1} a_{j}^{L}(t) \cdot x_{j}^{(U, 0)}(t) d \alpha+\int_{0}^{1} a_{j}^{U}(t) \cdot x_{j}^{(L, 0)}(t) d \alpha\right) \\
& \geq \sum_{i=1}^{p} \int_{0}^{1} c_{i}^{L}(t) \cdot y_{i}^{(I, n)}(t) d t+\sum_{i=1}^{p} \int_{0}^{1} c_{i}^{U}(t) \cdot y_{i}^{(I I, n)}(t) d t .
\end{aligned}
$$

Then $\mathbf{x}^{(0)}$ is an optimal solution of problem SOP. 
Proof. Since $\mathbf{x}^{(n)}$ is a feasible solution of problem SOP by Proposition 6 , for $i=1, \cdots, p, j=1, \cdots, q$ and $t \in[0,1]$, we have

$$
\begin{aligned}
& \sum_{\left\{j: j \in P_{i}\right\}} b_{i j}^{L}(t) \cdot x_{j}^{(L, n)}(t)+\sum_{\left\{j: j \in N_{i}\right\}} b_{i j}^{L}(t) \cdot x_{j}^{(U, n)}(t) \leq c_{i}^{L}(t) ; \\
& \sum_{\left\{j: j \in N_{i}\right\}} b_{i j}^{U}(t) \cdot x_{j}^{(L, n)}(t)+\sum_{\left\{j: j \in P_{i}\right\}} b_{i j}^{U}(t) \cdot x_{j}^{(U, n)}(t) \leq c_{i}^{U}(t) ; \\
& x_{j}^{(U, n)}(t) \geq x_{j}^{(L, n)}(t) \geq 0 ; \\
& \text { each } x_{j}^{(L, n)}(t) \text { is an increasing function on }[0,1] ; \\
& \text { each } x_{j}^{(U, n)}(t) \text { is a decreasing function on }[0,1] .
\end{aligned}
$$

For $i=1, \cdots, p$ and $t \in[0,1]$, we obtain

$$
\begin{aligned}
& \sum_{\left\{j: j \in P_{i}\right\}} b_{i j}^{L}(t) \cdot x_{j}^{(L, 0)}(t)+\sum_{\left\{j: j \in N_{i}\right\}} b_{i j}^{L}(t) \cdot x_{j}^{(U, 0)}(t) \\
& =\sum_{\left\{j: j \in P_{i}\right\}} b_{i j}^{L}(t) \cdot\left(\liminf _{n \rightarrow \infty} x_{j}^{(L, n)}(t)\right)+\sum_{\left\{j: j \in N_{i}\right\}} b_{i j}^{L}(t) \cdot\left(\liminf _{n \rightarrow \infty} x_{j}^{(U, n)}(t)\right) \\
& =\sum_{\left\{j: j \in P_{i}\right\}} \liminf _{n \rightarrow \infty}\left(b_{i j}^{L}(t) \cdot x_{j}^{(L, n)}(t)\right)+\sum_{\left\{j: j \in N_{i}\right\}} \liminf _{n \rightarrow \infty}\left(b_{i j}^{L}(t) \cdot x_{j}^{(U, n)}(t)\right) \\
& \leq \liminf _{n \rightarrow \infty}\left[\sum_{\left\{j: j \in P_{i}\right\}} b_{i j}^{L}(t) \cdot x_{j}^{(L, n)}(t)+\sum_{\left\{j: j \in N_{i}\right\}} b_{i j}^{L}(t) \cdot x_{j}^{(U, n)}(t)\right] \leq c_{i}^{L}(t) \text { (using (114)). }
\end{aligned}
$$

Using (115), for $i=1, \cdots, p$ and $t \in[0,1]$, we can similarly obtain

$$
\begin{aligned}
& \sum_{\left\{j: j \in N_{i}\right\}} b_{i j}^{U}(t) \cdot x_{j}^{(L, 0)}(t)+\sum_{\left\{j: j \in P_{i}\right\}} b_{i j}^{U}(t) \cdot x_{j}^{(U, 0)}(t) \\
& \quad \leq \liminf _{n \rightarrow \infty}\left[\sum_{\left\{j: j \in N_{i}\right\}} b_{i j}^{U}(t) \cdot x_{j}^{(L, n)}(t)+\sum_{\left\{j: j \in P_{i}\right\}} b_{i j}^{U}(t) \cdot x_{j}^{(U, n)}(t)\right] \leq c_{i}^{U}(t) .
\end{aligned}
$$

Using (116), for $j=1, \cdots, q$ and $t \in[0,1]$, we also have

$$
x_{j}^{(U, 0)}(t)=\liminf _{n \rightarrow \infty} x_{j}^{(U, n)}(t) \geq \liminf _{n \rightarrow \infty} x_{j}^{(L, n)}(t)=x_{j}^{(L, 0)}(t) \geq 0 .
$$

For $t_{1}<t_{2}$, using (117) and (118), we have

$$
x_{j}^{(L, n)}\left(t_{1}\right) \leq x_{j}^{(L, n)}\left(t_{2}\right) \text { and } x_{j}^{(U, n)}\left(t_{1}\right) \geq x_{j}^{(U, n)}\left(t_{2}\right),
$$

which imply

$$
x_{j}^{(L, 0)}\left(t_{1}\right)=\liminf _{n \rightarrow \infty} x_{j}^{(L, n)}\left(t_{1}\right) \leq \liminf _{n \rightarrow \infty} x_{j}^{(L, n)}\left(t_{2}\right)=x_{j}^{(L, 0)}\left(t_{2}\right)
$$

and

$$
x_{j}^{(U, 0)}\left(t_{1}\right)=\liminf _{n \rightarrow \infty} x_{j}^{(U, n)}\left(t_{1}\right) \geq \liminf _{n \rightarrow \infty} x_{j}^{(U, n)}\left(t_{2}\right)=x_{j}^{(U, 0)}\left(t_{2}\right) .
$$

Therefore $x_{j}^{(L, 0)}$ is an increasing function on $[0,1]$ and $x_{j}^{(U, 0)}$ is a decreasing function on $[0,1]$ for $j=1, \cdots, q$. This shows that $\mathbf{x}^{(0)}=\left(\mathbf{x}^{(L, 0)}, \mathbf{x}^{(U, 0)}\right)$ is indeed a feasible solution of problem SOP. 
Given any feasible solution $\left(\mathbf{x}^{L}, \mathbf{x}^{U}\right)$ of problem SOP, we have

$$
\begin{aligned}
& \sum_{j \in P}\left(\int_{0}^{1} a_{j}^{L}(t) \cdot x_{j}^{(L, 0)}(t) d \alpha+\int_{0}^{1} a_{j}^{U}(t) \cdot x_{j}^{(U, 0)}(t) d \alpha\right) \\
& \quad+\sum_{j \in N}\left(\int_{0}^{1} a_{j}^{L}(t) \cdot x_{j}^{(U, 0)}(t) d \alpha+\int_{0}^{1} a_{j}^{U}(t) \cdot x_{j}^{(L, 0)}(t) d \alpha\right) \\
& \geq \sum_{i=1}^{p} \int_{0}^{1} c_{i}^{L}(t) \cdot y_{i}^{(I, n)}(t) d t+\sum_{i=1}^{p} \int_{0}^{1} c_{i}^{U}(t) \cdot y_{i}^{(I I, n)}(t) d t(\text { using (113)) } \\
& \geq \sum_{j \in P}\left(\int_{0}^{1} a_{j}^{L}(t) \cdot x_{j}^{L}(t) d \alpha+\int_{0}^{1} a_{j}^{U}(t) \cdot x_{j}^{U}(t) d \alpha\right) \\
& \quad+\sum_{j \in N}\left(\int_{0}^{1} a_{j}^{L}(t) \cdot x_{j}^{U}(t) d \alpha+\int_{0}^{1} a_{j}^{U}(t) \cdot x_{j}^{L}(t) d \alpha\right) \quad(\text { using (31)), }
\end{aligned}
$$

which says that $\mathbf{x}^{(0)}=\left(\mathbf{x}^{(L, 0)}, \mathbf{x}^{(U, 0)}\right)$ is an optimal solution of problem SOP. This completes the proof.

In the sequel, we shall consider the uniform boundedness of the sequences $\left\{x_{j}^{(L, n)}\right\}_{n=1}^{\infty}$ and $\left\{x_{j}^{(U, n)}\right\}_{n=1}^{\infty}$ obtained from (62) and (63). Recall that part (i) of Proposition 5 has provided the sufficient conditions to guarantee the uniform boundedness of $\left\{x_{j}^{(L, n)}\right\}_{n=1}^{\infty}$ and $\left\{x_{j}^{(U, n)}\right\}_{n=1}^{\infty}$. In order to obtain the desired results, we also provide the different sufficient conditions below.

Proposition 8. Let $\mathbf{z}^{(n)}=\left(\mathbf{z}_{1}^{(n)}, \cdots, \mathbf{z}_{n}^{(n)}\right)$ with $\mathbf{z}_{l}^{(n)}=\left(\mathbf{z}_{l}^{(L, n)}, \mathbf{z}_{l}^{(U, n)}\right)$ for $l=1, \cdots, n$ be a feasible solution of primal problem $\left(\mathrm{P}_{n}\right)$. Suppose that $b_{\text {lij }}^{(L, n)}$ and $b_{l i j}^{(U, n)}$ are nonnegative for $i=1, \cdots, p, j=1, \cdots, q$ and $l=1, \cdots, n$ such that the following conditions are satisfied:

- $\quad$ for each $j=1, \cdots, q$ and $l=1, \cdots, n$, the following strict inequalities are satisfied:

$$
\sum_{i=1}^{p} b_{l i j}^{(L, n)}>0 \text { and } \sum_{i=1}^{p} b_{l i j}^{(U, n)}>0
$$

- $\quad$ there exist constants $\sigma^{L}>0$ and $\sigma^{U}>0$ such that, for each $i=1, \cdots, p, j=1, \cdots, q$ and $l=1, \cdots, n$, $b_{l i j}^{(L, n)} \neq 0$ implies $b_{l i j}^{(L, n)} \geq \sigma^{L}$ and $b_{l i j}^{(U, n)} \neq 0$ implies $b_{l i j}^{(U, n)} \geq \sigma^{U}$.

Then

$$
0 \leq z_{l j}^{(L, n)} \leq \frac{\mu^{L}}{\sigma^{L}} \text { and } 0 \leq z_{l j}^{(U, n)} \leq \frac{\mu^{U}}{\sigma^{U}},
$$

for all $j=1, \cdots, q$ and $l=1, \cdots, n$. In other words, the feasible set of primal problem $\left(\mathrm{P}_{n}\right)$ is uniformly bounded in the sense of saying that each feasible solution is bounded by some constant that is independent of $n$.

Proof. The assumptions say that, for each $j$ and $l$, there exists $i_{l j} \in\{1,2, \cdots, p\}$ such that $b_{l i_{l j} j}^{(L, n)} \geq$ $\sigma^{L}>0$. Then we have

$$
\begin{aligned}
0 & \leq \sigma^{L} \cdot z_{l j}^{(L, n)} \leq b_{l i_{l j} j}^{(L, n)} \cdot z_{l j}^{(L, n)} \leq \sum_{k=1}^{q} b_{l i_{l j} k}^{(L, n)} \cdot z_{l k}^{(L, n)} \text { (by the nonnegativity) } \\
& \left.\leq c_{l i_{l j}}^{(L, n)} \text { (by the feasibility of } \mathbf{z}^{(n)}\right) \\
& \leq \mu^{L} \text { (using (37)) }
\end{aligned}
$$


which implies

$$
0 \leq z_{l j}^{(L, n)} \leq \frac{\mu^{L}}{\sigma^{L}}
$$

We can similarly obtain

$$
0 \leq z_{l j}^{(U, n)} \leq \frac{\mu^{U}}{\sigma^{U}}
$$

This completes the proof.

For $i=1, \cdots, p$ and $j=1, \cdots, q$, we define the real-valued functions:

$$
\bar{a}_{j}^{(L, n)}(t)= \begin{cases}a_{l j}^{(L, n)} & \text { if } t \in F_{l}^{(n)} \text { for } l=1, \cdots, n \\ a_{n}^{(L, n)} & \text { if } t=1\end{cases}
$$

and

$$
\bar{a}_{j}^{(U, n)}(t)= \begin{cases}a_{l j}^{(U, n)} & \text { if } t \in F_{l}^{(n)} \text { for } l=1, \cdots, n \\ a_{n}^{(U, n)} & \text { if } t=1 .\end{cases}
$$

Lemma 5. Suppose that the sequences $\left\{x_{j}^{(L, n)}\right\}_{n=1}^{\infty}$ and $\left\{x_{j}^{(U, n)}\right\}_{n=1}^{\infty}$ obtained from (62) and (63) are uniformly bounded. For $i=1, \cdots, p$ and $j=1, \cdots, q$, we have

$$
\begin{aligned}
& \int_{0}^{1}\left[a_{j}^{L}(t)-\bar{a}_{j}^{(L, n)}(t)\right] \cdot x_{j}^{(L, n)}(t) d t \rightarrow 0 \text { as } n \rightarrow \infty \\
& \int_{0}^{1}\left[a_{j}^{L}(t)-\bar{a}_{j}^{(L, n)}(t)\right] \cdot x_{j}^{(U, n)}(t) d t \rightarrow 0 \text { as } n \rightarrow \infty \\
& \int_{0}^{1}\left[a_{j}^{U}(t)-\bar{a}_{j}^{(U, n)}(t)\right] \cdot x_{j}^{(L, n)}(t) d t \rightarrow 0 \text { as } n \rightarrow \infty \\
& \int_{0}^{1}\left[a_{j}^{U}(t)-\bar{a}_{j}^{(U, n)}(t)\right] \cdot x_{j}^{(U, n)}(t) d t \rightarrow 0 \text { as } n \rightarrow \infty
\end{aligned}
$$

Proof. It suffices to prove the case of (124). It is clear to see that the following function

$$
\left[a_{j}^{L}(t)-\bar{a}_{j}^{(L, n)}(t)\right] \cdot x_{j}^{(L, n)}(t)
$$

is continuous a.e. on $[0,1]$, i.e., it is Riemann-integrable on $[0,1]$. In other words, its Riemann integral and Lebesgue integral are identical. From Lemma 1 , for $j=1, \cdots, q, l=1, \cdots, n$ and $t \in F_{l}^{(n)}$, we see that

$$
0 \leq a_{j}^{L}(t)-\bar{a}_{j}^{(L, n)}(t)=a_{j}^{L}(t)-a_{l j}^{(L, n)} \leq \sup _{t \in F_{l}^{(n)}}\left[a_{j}^{L}(t)-a_{l j}^{(L, n)}\right] \rightarrow 0 \text { as } n \rightarrow \infty .
$$

Therefore we obtain

$$
a_{j}^{L}(t)-\bar{a}_{j}^{(L, n)}(t) \rightarrow 0 \text { as } n \rightarrow \infty \text { a.e. on }[0,1] .
$$

Since the sequence $\left\{x_{j}^{(L, n)}\right\}_{n=1}^{\infty}$ is uniformly bounded, using (128) and the Lebesgue bounded convergence theorem for integrals by referring to Royden [34], we can obtain (124). This completes the proof.

We remark that the uniform boundedness of the sequences $\left\{x_{j}^{(L, n)}\right\}_{n=1}^{\infty}$ and $\left\{x_{j}^{(U, n)}\right\}_{n=1}^{\infty}$ in Lemma 5 can be guaranteed by applying Proposition 8 and part (i) of Proposition 5. 
Lemma 6. (Fatou's Lemma) (Royden [34]) Let $\left\{f_{k}\right\}_{k=1}^{\infty}$ be a sequence of Lebesgue measurable functions defined on the same Lebesgue measurable set $E$, and let $v$ be a Lebesgue measure on $E$.

(i) If there exists a Lebesgue integrable function $\phi$ on $E$ such that $f_{k} \geq \phi$ a.e. on $E$ for all $k$, then

$$
\int_{E}\left(\liminf _{k \rightarrow \infty} f_{k}\right) d v \leq \liminf _{k \rightarrow \infty} \int_{E} f_{k} d v
$$

(ii) If there exists a Lebesgue integrable function $\phi$ on $E$ such that $f_{k} \leq \phi$ a.e. on $E$ for all $k$, then

$$
\int_{E}\left(\limsup _{k \rightarrow \infty} f_{k}\right) d v \geq \limsup _{k \rightarrow \infty} \int_{E} f_{k} d v
$$

Theorem 7. Given an optimal solution $\mathbf{z}^{(* n)}$ of primal problem $\left(\mathrm{P}_{n}\right)$ and an optimal solution $\mathbf{w}^{(* n)}$ of dual problem $\left(\mathrm{D}_{n}\right)$, we define

$$
\mathbf{x}^{(n)}=\left(\mathbf{x}^{(L, n)}, \mathbf{x}^{(U, n)}\right) \text { and } \mathbf{y}^{(n)}(t)=\left(\mathbf{y}^{(I, n)}(t), \mathbf{y}^{(I I, n)}(t), \mathbf{y}^{(I I I, n)}(t), \mathbf{y}^{(I V, n)}(t), \mathbf{y}^{(V, n)}(t)\right)
$$

according to (62) and (63) and (88)-(92), respectively. Considering the component functions $x_{j}^{(L, n)}$ and $x_{j}^{(U, n)}$ of $\mathbf{x}^{(L, n)}$ and $\mathbf{x}^{(U, n)}$, respectively, for $j=1, \cdots, q$, we assume that the sequences $\left\{x_{j}^{(L, n)}\right\}_{n=1}^{\infty}$ and $\left\{x_{j}^{(U, n)}\right\}_{n=1}^{\infty}$ are uniformly bounded and satisfy the following inequalities

$$
\begin{aligned}
& \sum_{\left\{j: j \in P_{i}\right\}} b_{i j}^{L}(t) \cdot\left(\limsup _{n \rightarrow \infty} x_{j}^{(L, n)}(t)\right)+\sum_{\left\{j: j \in N_{i}\right\}} b_{i j}^{L}(t) \cdot\left(\limsup _{n \rightarrow \infty} x_{j}^{(U, n)}(t)\right) \\
& \leq \limsup _{n \rightarrow \infty}\left[\sum_{\left\{j: j \in P_{i}\right\}} b_{i j}^{L}(t) \cdot x_{j}^{(L, n)}(t)+\sum_{\left\{j: j \in N_{i}\right\}} b_{i j}^{L}(t) \cdot x_{j}^{(U, n)}(t)\right]
\end{aligned}
$$

and

$$
\begin{aligned}
& \sum_{\left\{j: j \in N_{i}\right\}} b_{i j}^{U}(t) \cdot\left(\limsup _{n \rightarrow \infty} x_{j}^{(L, n)}(t)\right)+\sum_{\left\{j: j \in P_{i}\right\}} b_{i j}^{U}(t) \cdot\left(\limsup _{n \rightarrow \infty} x_{j}^{(U, n)}(t)\right) \\
& \leq \limsup _{n \rightarrow \infty}\left[\sum_{\left\{j: j \in N_{i}\right\}} b_{i j}^{U}(t) \cdot x_{j}^{(L, n)}(t)+\sum_{\left\{j: j \in P_{i}\right\}} b_{i j}^{U}(t) \cdot x_{j}^{(U, n)}(t)\right]
\end{aligned}
$$

for $i=1, \cdots, p$. Now we define

$$
x_{j}^{(L, 0)}(t)=\limsup _{n \rightarrow \infty} x_{j}^{(L, n)}(t) \text { and } x_{j}^{(U, 0)}(t)=\limsup _{n \rightarrow \infty} x_{j}^{(U, n)}(t)
$$

for $j=1, \cdots, q$ and $t \in[0,1]$. Then $\mathbf{x}^{(0)}=\left(\mathbf{x}^{(L, 0)}, \mathbf{x}^{(U, 0)}\right)$ with the component functions $x_{j}^{(L, 0)}$ and $x_{j}^{(U, 0)}$ of $\mathbf{x}^{(L, 0)}$ and $\mathbf{x}^{(U, 0)}$, respectively, for $j=1, \cdots, q$, is an optimal solution of problem SOP. 
Proof. For $i=1, \cdots, p$ and $t \in[0,1]$, we have

$$
\begin{aligned}
& \sum_{\left\{j: j \in P_{i}\right\}} b_{i j}^{L}(t) \cdot x_{j}^{(L, 0)}(t)+\sum_{\left\{j: j \in N_{i}\right\}} b_{i j}^{L}(t) \cdot x_{j}^{(U, 0)}(t) \\
& =\sum_{\left\{j: j \in P_{i}\right\}} b_{i j}^{L}(t) \cdot\left(\limsup _{n \rightarrow \infty} x_{j}^{(L, n)}(t)\right)+\sum_{\left\{j: j \in N_{i}\right\}} b_{i j}^{L}(t) \cdot\left(\limsup _{n \rightarrow \infty} x_{j}^{(U, n)}(t)\right) \\
& \leq \limsup _{n \rightarrow \infty}\left[\sum_{\left\{j: j \in P_{i}\right\}} b_{i j}^{L}(t) \cdot x_{j}^{(L, n)}(t)+\sum_{\left\{j: j \in N_{i}\right\}} b_{i j}^{L}(t) \cdot x_{j}^{(U, n)}(t)\right](\operatorname{using}(129)) \\
& \leq c_{i}^{L}(t) \text { (using (114)). }
\end{aligned}
$$

For $i=1, \cdots, p$ and $t \in[0,1]$, using (115) and (130), we can similarly obtain

$$
\sum_{\left\{j: j \in N_{i}\right\}} b_{i j}^{U}(t) \cdot x_{j}^{(L, 0)}(t)+\sum_{\left\{j: j \in P_{i}\right\}} b_{i j}^{U}(t) \cdot x_{j}^{(U, 0)}(t) \leq c_{i}^{U}(t) .
$$

Applying the limit superior to the argument in the proof of Theorem 6, we can also show that $\mathbf{x}^{(0)}=\left(\mathbf{x}^{(L, 0)}, \mathbf{x}^{(U, 0)}\right)$ is a feasible solution of problem SOP.

We remain to prove the optimality. We first have

$$
\begin{aligned}
& \sum_{j \in P} \int_{0}^{1} a_{j}^{L}(t) \cdot x_{j}^{(L, n)}(t) d t \\
& \quad=\sum_{j \in P} \sum_{l=1}^{n} \int_{\bar{E}_{l}^{(n)}}\left(a_{j}^{L}(t)-a_{j l}^{(L, n)}\right) \cdot x_{j}^{(L, n)}(t) d t+\sum_{j \in P} \sum_{l=1}^{n} \int_{\bar{E}_{l}^{(n)}} a_{j l}^{(L, n)} \cdot x_{j}^{(L, n)}(t) d t \\
& \quad=\sum_{j \in P} \sum_{l=1}^{n} \int_{\bar{E}_{l}^{(n)}}\left(a_{j}^{L}(t)-a_{j l}^{(L, n)}\right) \cdot z_{l j}^{(* L, n)} d t+\sum_{j \in P} \sum_{l=1}^{n} \frac{1}{n} \cdot a_{j l}^{(L, n)} \cdot z_{l j}^{(* L, n)} \\
& \quad \equiv \mathfrak{a}^{(P L, n)}+\sum_{j \in P} \sum_{l=1}^{n} \frac{1}{n} \cdot a_{j l}^{(L, n)} \cdot z_{l j}^{(* L, n)},
\end{aligned}
$$

where

$$
\mathfrak{a}^{(P L, n)}=\sum_{j \in P} \sum_{l=1}^{n} \int_{\bar{E}_{l}^{(n)}}\left(a_{j}^{L}(t)-a_{j l}^{(L, n)}\right) \cdot z_{l j}^{(* L, n)} d t
$$

and similarly have

$$
\begin{aligned}
& \sum_{j \in N} \int_{0}^{1} a_{j}^{L}(t) \cdot x_{j}^{(U, n)}(t) d t=\mathfrak{a}^{(N U, n)}+\sum_{j \in N} \sum_{l=1}^{n} \frac{1}{n} \cdot a_{j l}^{(L, n)} \cdot z_{l j}^{(* U, n)} \\
& \sum_{j \in N} \int_{0}^{1} a_{j}^{U}(t) \cdot x_{j}^{(L, n)}(t) d t=\mathfrak{a}^{(N L, n)}+\sum_{j \in N} \sum_{l=1}^{n} \frac{1}{n} \cdot a_{j l}^{(U, n)} \cdot z_{l j}^{(* L, n)} \\
& \sum_{j \in P} \int_{0}^{1} a_{j}^{U}(t) \cdot x_{j}^{(U, n)}(t) d t=\mathfrak{a}^{(P U, n)}+\sum_{j \in P} \sum_{l=1}^{n} \frac{1}{n} \cdot a_{j l}^{(U, n)} \cdot z_{l j}^{(* U, n),}
\end{aligned}
$$

where

$$
\begin{aligned}
& \mathfrak{a}^{(N U, n)}=\sum_{j \in N} \sum_{l=1}^{n} \int_{\bar{E}_{l}^{(n)}}\left(a_{j}^{L}(t)-a_{j l}^{(L, n)}\right) \cdot z_{l j}^{(* U, n)} d t \\
& \mathfrak{a}^{(N L, n)}=\sum_{j \in N} \sum_{l=1}^{n} \int_{\bar{E}_{l}^{(n)}}\left(a_{j}^{U}(t)-a_{j l}^{(U, n)}\right) \cdot z_{l j}^{(* L, n)} d t
\end{aligned}
$$




$$
\mathfrak{a}^{(P U, n)}=\sum_{j \in P} \sum_{l=1}^{n} \int_{\bar{E}_{l}^{(n)}}\left(a_{j}^{U}(t)-a_{j l}^{(U, n)}\right) \cdot z_{l j}^{(* U, n)} d t .
$$

\section{Then we obtain}

$$
\begin{aligned}
& 0 \leq \mathfrak{a}^{(P L, n)}=\sum_{j \in P} \sum_{l=1}^{n} \int_{F_{l}^{(n)}}\left(a_{j}^{L}(t)-a_{j l}^{(L, n)}\right) \cdot z_{l j}^{(* L, n)} d t \\
&=\sum_{j \in P} \int_{0}^{1}\left[a_{j}^{L}(t)-\bar{a}_{j}^{(L, n)}(t)\right] \cdot x_{j}^{(L, n)}(t) d t \rightarrow 0 \text { as } n \rightarrow \infty \text { (using (122) and (124)) } \\
& 0 \leq \mathfrak{a}^{(N U, n)}=\sum_{j \in P} \sum_{l=1}^{n} \int_{F_{l}^{(n)}}\left(a_{j}^{L}(t)-a_{j l}^{(L, n)}\right) \cdot z_{l j}^{(* U, n)} d t \\
& \leq \sum_{j \in N} \int_{0}^{1}\left[a_{j}^{L}(t)-\bar{a}_{j}^{(L, n)}(t)\right] \cdot x_{j}^{(U, n)}(t) d t \rightarrow 0 \text { as } n \rightarrow \infty \text { (using (122) and (125)) } \\
& 0 \leq \mathfrak{a}^{(N L, n)}=\sum_{j \in P} \sum_{l=1}^{n} \int_{F_{l}^{(n)}}\left(a_{j}^{U}(t)-a_{j l}^{(U, n)}\right) \cdot z_{l j}^{(* L, n)} d t \\
& \leq \sum_{j \in N} \int_{0}^{1}\left[a_{j}^{U}(t)-\bar{a}_{j}^{(U, n)}(t)\right] \cdot x_{j}^{(L, n)}(t) d t \rightarrow 0 \text { as } n \rightarrow \infty \text { (using (123) and (126)) } \\
& 0 \leq \mathfrak{a}^{(P U, n)}=\sum_{j \in P} \sum_{l=1}^{n} \int_{F_{l}^{(n)}}\left(a_{j}^{U}(t)-a_{j l}^{(U, n)}\right) \cdot z_{l j}^{(* U, n)} d t \\
& \leq \sum_{j \in P} \int_{0}^{1}\left[a_{j}^{U}(t)-\bar{a}_{j}^{(U, n)}(t)\right] \cdot x_{j}^{(U, n)}(t) d t \rightarrow 0 \text { as } n \rightarrow \infty . \text { (using (123) and (127)). }
\end{aligned}
$$

By adding (132)-(135) together, we also obtain

$$
\begin{gathered}
\sum_{j \in P}\left(\int_{0}^{1} a_{j}^{L}(t) \cdot x_{j}^{(L, n)}(t) d \alpha+\int_{0}^{1} a_{j}^{U}(t) \cdot x_{j}^{(U, n)}(t) d \alpha\right) \\
\quad+\sum_{j \in N}\left(\int_{0}^{1} a_{j}^{L}(t) \cdot x_{j}^{(U, n)}(t) d \alpha+\int_{0}^{1} a_{j}^{U}(t) \cdot x_{j}^{(L, n)}(t) d \alpha\right) \\
=\mathfrak{a}^{(P L, n)}+\mathfrak{a}^{(N U, n)}+\mathfrak{a}^{(N L, n)}+\mathfrak{a}^{(P U, n)}+V\left(\mathrm{P}_{n}\right) .
\end{gathered}
$$


On the other hand, we have

$$
\begin{aligned}
\sum_{i=1}^{p} & \int_{0}^{1} c_{i}^{L}(t) \cdot y_{i}^{(I, n)}(t) d t \\
= & \sum_{i=1}^{p} \sum_{l=1}^{n} \int_{\bar{E}_{l}^{(n)}}\left(c_{i}^{L}(t)-c_{l i}^{(L, n)}\right) \cdot y_{i}^{(I, n)}(t) d t+\sum_{i=1}^{p} \sum_{l=1}^{n} \int_{\bar{E}_{l}^{(n)}} c_{l i}^{(L, n)} \cdot y_{i}^{(I, n)}(t) d t \\
= & \sum_{i=1}^{p} \sum_{l=1}^{n} \int_{\bar{E}_{l}^{(n)}}\left(c_{i}^{L}(t)-c_{l i}^{(L, n)}\right) \cdot w_{l i}^{(* I, n)} d t+\sum_{i=1}^{p} \frac{1}{n} \cdot c_{l i}^{(L, n)} \cdot w_{l i}^{(* I, n)} \\
& +\sum_{i=1}^{p} \sum_{l=1}^{n} \int_{\bar{E}_{l}^{(n)}} c_{i}^{L}(t) \cdot \max \left\{\mathfrak{f}^{(P N, n)}(t), \mathfrak{f}^{(N P, n)}(t)\right\} d t \\
\equiv & \mathfrak{c}^{(L, n)}+\sum_{i=1}^{p} \frac{1}{n} \cdot c_{l i}^{(L, n)} \cdot w_{l i}^{(* I, n)}
\end{aligned}
$$

and

$$
\sum_{i=1}^{p} \int_{0}^{1} c_{i}^{U}(t) \cdot y_{i}^{(I I, n)}(t) d t=\mathfrak{c}^{(U, n)}+\sum_{i=1}^{p} \frac{1}{n} \cdot c_{l i}^{(U, n)} \cdot w_{l i}^{(* I I, n)}
$$

where

$$
\begin{aligned}
\mathfrak{c}^{(L, n)} & =\sum_{i=1}^{p} \sum_{l=1}^{n} \int_{\bar{E}_{l}^{(n)}}\left(c_{i}^{L}(t)-c_{l i}^{(L, n)}\right) \cdot w_{l i}^{(* I, n)} d t \\
& +\sum_{i=1}^{p} \sum_{l=1}^{n} \int_{\bar{E}_{l}^{(n)}} c_{i}^{L}(t) \cdot \max \left\{\mathfrak{f}^{(P N, n)}(t), \mathfrak{f}^{(N P, n)}(t)\right\} d t
\end{aligned}
$$

and

$$
\begin{aligned}
\mathfrak{c}^{(U, n)} & =\sum_{i=1}^{p} \sum_{l=1}^{n} \int_{\bar{E}_{l}^{(n)}}\left(c_{i}^{U}(t)-c_{l i}^{(U, n)}\right) \cdot w_{l i}^{(* I I, n)} d t \\
& +\sum_{i=1}^{p} \sum_{l=1}^{n} \int_{\bar{E}_{l}^{(n)}} c_{i}^{U}(t) \cdot \max \left\{\mathfrak{f}^{(P N, n)}(t), \mathfrak{f}^{(N P, n)}(t)\right\} d t .
\end{aligned}
$$

We obtain

$$
\begin{aligned}
0 & \leq \sum_{i=1}^{p} \sum_{l=1}^{n} \int_{\bar{E}_{l}^{(n)}}\left(c_{i}^{L}(t)-c_{l i}^{(L, n)}\right) \cdot w_{l i}^{(* I, n)} d t \\
& =\sum_{i=1}^{p} \int_{0}^{1}\left[c_{i}^{L}(t)-\bar{c}_{i}^{(L, n)}(t)\right] \cdot \bar{w}_{i}^{(* I, n)}(t) d t \rightarrow 0 \text { as } n \rightarrow \infty(\operatorname{using}(97),(99) \text { and (101)) }
\end{aligned}
$$

and

$$
\begin{aligned}
0 & \leq \sum_{i=1}^{p} \sum_{l=1}^{n} \int_{\bar{E}_{l}^{(n)}}\left(c_{i}^{U}(t)-c_{l i}^{(U, n)}\right) \cdot w_{l i}^{(* I I, n)} d t \\
& =\sum_{i=1}^{p} \int_{0}^{1}\left[c_{i}^{U}(t)-\bar{c}_{i}^{(U, n)}(t)\right] \cdot \bar{w}_{i}^{(* I I, n)}(t) d t \rightarrow 0 \text { as } n \rightarrow \infty \text { (using (98), (100) and (102)). }
\end{aligned}
$$

From (143) and (144), using (145), (146), (110) and (111), it follows that

$$
\mathfrak{c}^{(L, n)} \rightarrow 0 \text { and } \mathfrak{c}^{(U, n)} \rightarrow 0 \text { as } n \rightarrow \infty .
$$


Mathematics 2019, 7, 569

53 of 105

By adding (141) and (142) together, we obtain

$$
\sum_{i=1}^{p} \int_{0}^{1} c_{i}^{L}(t) \cdot y_{i}^{(I, n)}(t) d t+\sum_{i=1}^{p} \int_{0}^{1} c_{i}^{U}(t) \cdot y_{i}^{(I I, n)}(t) d t=\mathfrak{c}^{(L, n)}+\mathfrak{c}^{(U, n)}+V\left(\mathrm{D}_{n}\right) .
$$

Since $V\left(\mathrm{P}_{n}\right)=V\left(\mathrm{D}_{n}\right)$, from (140) and (148), we have

$$
\begin{aligned}
\sum_{j \in P}\left(\int_{0}^{1} a_{j}^{L}(t) \cdot x_{j}^{(L, n)}(t) d \alpha+\int_{0}^{1} a_{j}^{U}(t) \cdot x_{j}^{(U, n)}(t) d \alpha\right) \\
\quad+\sum_{j \in N}\left(\int_{0}^{1} a_{j}^{L}(t) \cdot x_{j}^{(U, n)}(t) d \alpha+\int_{0}^{1} a_{j}^{U}(t) \cdot x_{j}^{(L, n)}(t) d \alpha\right) \\
=\sum_{i=1}^{p} \int_{0}^{1} c_{i}^{L}(t) \cdot y_{i}^{(I, n)}(t) d t+\sum_{i=1}^{p} \int_{0}^{1} c_{i}^{U}(t) \cdot y_{i}^{(I I, n)}(t) d t \\
\quad+\mathfrak{a}^{(P L, n)}+\mathfrak{a}^{(N U, n)}+\mathfrak{a}^{(N L, n)}+\mathfrak{a}^{(P U, n)}-\mathfrak{c}^{(L, n)}-\mathfrak{c}^{(U, n)} .
\end{aligned}
$$

Given any feasible solution $\left(\mathbf{x}^{L}, \mathbf{x}^{U}\right)$ of problem SOP, using (31) and (149), we have

$$
\begin{aligned}
\sum_{j \in P}\left(\int_{0}^{1} a_{j}^{L}(t) \cdot x_{j}^{(L, n)}(t) d \alpha+\int_{0}^{1} a_{j}^{U}(t) \cdot x_{j}^{(U, n)}(t) d \alpha\right) \\
+\sum_{j \in N}\left(\int_{0}^{1} a_{j}^{L}(t) \cdot x_{j}^{(U, n)}(t) d \alpha+\int_{0}^{1} a_{j}^{U}(t) \cdot x_{j}^{(L, n)}(t) d \alpha\right) \\
\geq \sum_{j \in P}\left(\int_{0}^{1} a_{j}^{L}(t) \cdot x_{j}^{L}(t) d \alpha+\int_{0}^{1} a_{j}^{U}(t) \cdot x_{j}^{U}(t) d \alpha\right) \\
+\sum_{j \in N}\left(\int_{0}^{1} a_{j}^{L}(t) \cdot x_{j}^{U}(t) d \alpha+\int_{0}^{1} a_{j}^{U}(t) \cdot x_{j}^{L}(t) d \alpha\right) \\
+\mathfrak{a}^{(P L, n)}+\mathfrak{a}^{(N U, n)}+\mathfrak{a}^{(N L, n)}+\mathfrak{a}^{(P U, n)}-\mathfrak{c}^{(L, n)}-\mathfrak{c}^{(U, n) .}
\end{aligned}
$$

Using the Fatou's Lemma 6, we have

$$
\begin{aligned}
\int_{0}^{1} a_{j}^{L}(t) \cdot\left(\limsup _{n \rightarrow \infty} x_{j}^{(L, n)}(t)\right) d t & =\int_{0}^{1} \limsup _{n \rightarrow \infty}\left[a_{j}^{L}(t) \cdot x_{j}^{(L, n)}(t)\right] d t \\
& \geq \limsup _{n \rightarrow \infty} \int_{0}^{1} a_{j}^{L}(t) \cdot x_{j}^{(L, n)}(t) d t \text { (by Fatou's lemma) }
\end{aligned}
$$

and

$$
\begin{aligned}
& \int_{0}^{1} a_{j}^{L}(t) \cdot\left(\limsup _{n \rightarrow \infty} x_{j}^{(U, n)}(t)\right) d t \geq \limsup _{n \rightarrow \infty} \int_{0}^{1} a_{j}^{L}(t) \cdot x_{j}^{(U, n)}(t) d t \\
& \int_{0}^{1} a_{j}^{U}(t) \cdot\left(\limsup _{n \rightarrow \infty} x_{j}^{(L, n)}(t)\right) d t \geq \limsup _{n \rightarrow \infty} \int_{0}^{1} a_{j}^{U}(t) \cdot x_{j}^{(L, n)}(t) d t \\
& \int_{0}^{1} a_{j}^{U}(t) \cdot\left(\limsup _{n \rightarrow \infty} x_{j}^{(U, n)}(t)\right) d t \geq \limsup _{n \rightarrow \infty} \int_{0}^{1} a_{j}^{U}(t) \cdot x_{j}^{(U, n)}(t) d t
\end{aligned}
$$

Then we obtain

$$
\begin{aligned}
\sum_{j \in P}\left(\int_{0}^{1} a_{j}^{L}(t) \cdot x_{j}^{(L, 0)}(t) d \alpha\right. & \left.+\int_{0}^{1} a_{j}^{U}(t) \cdot x_{j}^{(U, 0)}(t) d \alpha\right) \\
& +\sum_{j \in N}\left(\int_{0}^{1} a_{j}^{L}(t) \cdot x_{j}^{(U, 0)}(t) d \alpha+\int_{0}^{1} a_{j}^{U}(t) \cdot x_{j}^{(L, 0)}(t) d \alpha\right)
\end{aligned}
$$




$$
\begin{aligned}
& =\sum_{j \in P} \int_{0}^{1} a_{j}^{L}(t) \cdot\left(\limsup _{n \rightarrow \infty} x_{j}^{(L, n)}(t)\right) d t+\sum_{j \in N} \int_{0}^{1} a_{j}^{L}(t) \cdot\left(\limsup _{n \rightarrow \infty} x_{j}^{(U, n)}(t)\right) d t \\
& +\sum_{j \in N} \int_{0}^{1} a_{j}^{U}(t) \cdot\left(\limsup _{n \rightarrow \infty} x_{j}^{(L, n)}(t)\right) d t+\sum_{j \in P} \int_{0}^{1} a_{j}^{U}(t) \cdot\left(\limsup _{n \rightarrow \infty} x_{j}^{(U, n)}(t)\right) d t \\
& \geq \sum_{j \in P}\left(\limsup _{n \rightarrow \infty} \int_{0}^{1} a_{j}^{L}(t) \cdot x_{j}^{(L, n)}(t) d t\right)+\sum_{j \in N}\left(\limsup _{n \rightarrow \infty} \int_{0}^{1} a_{j}^{L}(t) \cdot x_{j}^{(U, n)}(t) d t\right) \\
& +\sum_{j \in N}\left(\limsup _{n \rightarrow \infty} \int_{0}^{1} a_{j}^{U}(t) \cdot x_{j}^{(L, n)}(t) d t\right)+\sum_{j \in P}\left(\limsup _{n \rightarrow \infty} \int_{0}^{1} a_{j}^{U}(t) \cdot x_{j}^{(U, n)}(t) d t\right) \\
& \text { (using (151)-(154)) } \\
& \geq \limsup _{n \rightarrow \infty}\left[\sum_{j \in P}\left(\int_{0}^{1} a_{j}^{L}(t) \cdot x_{j}^{(L, n)}(t) d \alpha+\int_{0}^{1} a_{j}^{U}(t) \cdot x_{j}^{(U, n)}(t) d \alpha\right)\right. \\
& \left.+\sum_{j \in N}\left(\int_{0}^{1} a_{j}^{L}(t) \cdot x_{j}^{(U, n)}(t) d \alpha+\int_{0}^{1} a_{j}^{U}(t) \cdot x_{j}^{(L, n)}(t) d \alpha\right)\right] \\
& \geq \sum_{j \in P}\left(\int_{0}^{1} a_{j}^{L}(t) \cdot x_{j}^{L}(t) d \alpha+\int_{0}^{1} a_{j}^{U}(t) \cdot x_{j}^{U}(t) d \alpha\right) \\
& +\sum_{j \in N}\left(\int_{0}^{1} a_{j}^{L}(t) \cdot x_{j}^{U}(t) d \alpha+\int_{0}^{1} a_{j}^{U}(t) \cdot x_{j}^{L}(t) d \alpha\right) \\
& +\limsup _{n \rightarrow \infty}\left(\mathfrak{a}^{(P L, n)}+\mathfrak{a}^{(N U, n)}+\mathfrak{a}^{(N L, n)}+\mathfrak{a}^{(P U, n)}-\mathfrak{c}^{(L, n)}-\mathfrak{c}^{(U, n)}\right)(\operatorname{using}(150)) \\
& =\sum_{j \in P}\left(\int_{0}^{1} a_{j}^{L}(t) \cdot x_{j}^{L}(t) d \alpha+\int_{0}^{1} a_{j}^{U}(t) \cdot x_{j}^{U}(t) d \alpha\right) \\
& +\sum_{j \in N}\left(\int_{0}^{1} a_{j}^{L}(t) \cdot x_{j}^{U}(t) d \alpha+\int_{0}^{1} a_{j}^{U}(t) \cdot x_{j}^{L}(t) d \alpha\right) \\
& \text { (using (136)-(139) and (147)) }
\end{aligned}
$$

Since $\left(\mathbf{x}^{L}, \mathbf{x}^{U}\right)$ can be any feasible solution of problem SOP, it shows that $\left(\mathbf{x}^{(L, 0)}, \mathbf{x}^{(U, 0)}\right)$ is an optimal solution of problem SOP. This completes the proof.

In the sequel, we shall present the existence of optimal solutions by considering the subsequences. We first provide some useful lemmas.

Lemma 7. (Riesz and Sz.-Nagy ([35] p. 64)) Let $\left\{f_{k}\right\}_{k=1}^{\infty}$ be a sequence in $L^{2}[0,1]$. If the sequence $\left\{f_{k}\right\}_{k=1}^{\infty}$ is uniformly bounded with respect to $\|\cdot\|_{2}$, then exists a subsequence $\left\{f_{k_{j}}\right\}_{j=1}^{\infty}$ which weakly converges to $f \in L^{2}[0,1]$. In other words, for any $g \in L^{2}[0,1]$, we have

$$
\lim _{j \rightarrow \infty} \int_{0}^{T} f_{k_{j}}(t) \cdot g(t) d t=\int_{0}^{T} f(t) \cdot g(t) d t .
$$

We remark that if the sequences $\left\{x_{j}^{(L, n)}\right\}_{n=1}^{\infty}$ and $\left\{x_{j}^{(U, n)}\right\}_{n=1}^{\infty}$ are uniformly bounded, then they are also uniformly bounded with respect to $\|\cdot\|_{2}$. 
Lemma 8. (Levinson [36]) If the sequence $\left\{f_{k}\right\}_{k=1}^{\infty}$ is uniformly bounded on $[0,1]$ with respect to $\|\cdot\|_{2}$ and weakly converges to $f \in L^{2}[0,1]$, then

$$
f(t) \leq \limsup _{k \rightarrow \infty} f_{k}(t) \text { and } f(t) \geq \liminf _{k \rightarrow \infty} f_{k}(t) \text { a.e. on }[0,1] .
$$

Lemma 9. Let $\left\{f_{k}\right\}_{k=1}^{\infty}$ and $\left\{g_{k}\right\}_{k=1}^{\infty}$ be two sequences in $L^{2}[0,1]$ that weakly converge to $f_{0}$ and $g_{0}$ in $L^{2}[0,1]$, respectively.

(i) If the function $\eta$ defined on $[0,1]$ is bounded, then the sequence $\left\{\eta \cdot f_{k}\right\}_{k=1}^{\infty}$ weakly converges to $\eta \cdot f_{0}$.

(ii) The sequence $\left\{f_{k}+g_{k}\right\}_{k=1}^{\infty}$ weakly converges to $f_{0}+g_{0}$.

Proof. To prove part (i), for any $h \in L^{2}[0,1]$, we see that $h \cdot \eta \in L^{2}[0,1]$. Therefore the weak convergence says that

$$
\lim _{k \rightarrow \infty} \int_{0}^{T} h \cdot\left(\eta \cdot f_{k}\right) d t=\lim _{k \rightarrow \infty} \int_{0}^{T}(h \cdot \eta) \cdot f_{k} d t=\int_{0}^{T}(h \cdot \eta) \cdot f_{0} d t=\int_{0}^{T} h \cdot\left(\eta \cdot f_{0}\right) d t,
$$

which says that the sequence $\left\{\eta \cdot f_{k}\right\}_{k=1}^{\infty}$ weakly converges to $\eta \cdot f_{0}$.

To prove part (ii), for any $h \in L^{2}[0,1]$, we have

$$
\begin{aligned}
\lim _{k \rightarrow \infty} & \int_{0}^{T}\left(f_{k}(t)+g_{k}(t)\right) \cdot h(t) d t=\lim _{k \rightarrow \infty}\left(\int_{0}^{T} f_{k}(t) \cdot h(t) d t+\int_{0}^{T} g_{k}(t) \cdot h(t) d t\right) \\
= & \int_{0}^{T} f_{0}(t) \cdot h(t) d t+\int_{0}^{T} g_{0}(t) \cdot h(t) d t \\
& \left.\quad \text { by the weak convergence for the sequences }\left\{f_{k}\right\}_{k=1}^{\infty} \text { and }\left\{g_{k}\right\}_{k=1}^{\infty}\right) \\
= & \int_{0}^{T}\left(f_{0}(t)+g_{0}(t)\right) \cdot h(t) d t .
\end{aligned}
$$

This completes the proof.

Theorem 8. Given an optimal solution $\mathbf{z}^{(* n)}$ of primal problem $\left(\mathrm{P}_{n}\right)$ and an optimal solution $\mathbf{w}^{(* n)}$ of dual problem $\left(\mathrm{D}_{n}\right)$, we define

$$
\mathbf{x}^{(n)}=\left(\mathbf{x}^{(L, n)}, \mathbf{x}^{(U, n)}\right) \text { and } \mathbf{y}^{(n)}(t)=\left(\mathbf{y}^{(I, n)}(t), \mathbf{y}^{(I I, n)}(t), \mathbf{y}^{(I I I, n)}(t), \mathbf{y}^{(I V, n)}(t), \mathbf{y}^{(V, n)}(t)\right)
$$

according to (62) and (63) and (88)-(92), respectively. Considering the component functions $x_{j}^{(L, n)}$ and $x_{j}^{(U, n)}$ of $\mathbf{x}^{(L, n)}$ and $\mathbf{x}^{(U, n)}$, respectively, for $j=1, \cdots, q$, we assume that the sequences $\left\{x_{j}^{(L, n)}\right\}_{n=1}^{\infty}$ and $\left\{x_{j}^{(U, n)}\right\}_{n=1}^{\infty}$ are uniformly bounded with respect to $\|\cdot\|_{2}$ and satisfy the following inequalities:

$$
\begin{gathered}
\liminf _{n \rightarrow \infty} x_{j}^{(U, n)}(t) \geq \limsup _{n \rightarrow \infty} x_{j}^{(L, n)}(t) \text { for } t \in[0,1] \\
\liminf _{n \rightarrow \infty} x_{j}^{(L, n)}\left(t_{2}\right) \geq \limsup _{n \rightarrow \infty} x_{j}^{(L, n)}\left(t_{1}\right) \text { for } t_{1}, t_{2} \in[0,1] \text { with } t_{1}<t_{2} \\
\liminf _{n \rightarrow \infty}^{(U, n)}\left(t_{1}\right) \geq \limsup _{n \rightarrow \infty} x_{j}^{(U, n)}\left(t_{2}\right) \text { for } t_{1}, t_{2} \in[0,1] \text { with } t_{1}<t_{2}
\end{gathered}
$$

for $j=1, \cdots, q$. Then there is a subsequence $\left\{\mathbf{x}^{\left(n_{k}\right)}\right\}_{k=1}^{\infty}$ of $\left\{\mathbf{x}^{(n)}\right\}_{n=1}^{\infty}$ such that $\left\{\mathbf{x}^{\left(n_{k}\right)}\right\}_{k=1}^{\infty}$ is weakly convergent to an optimal solution $\mathbf{x}^{*}=\left(\mathbf{x}^{(* L)}, \mathbf{x}^{(* U)}\right)$ of problem SOP.

Proof. Since the sequence $\left\{x_{1}^{(L, n)}\right\}_{n=1}^{\infty}$ is uniformly bounded with respect to $\|\cdot\|_{2}$, using Lemma 7 , there exists a subsequence $\left\{x_{1}^{\left(L, n_{k}^{(1)}\right)}\right\}_{k=1}^{\infty}$ of $\left\{x_{1}^{(L, n)}\right\}_{n=1}^{\infty}$ that weakly converges to some $x_{1}^{(L, 0)} \in L^{2}[0,1]$. 
Using Lemma 7 again, there exists a subsequence $\left\{x_{2}^{\left(L, n_{k}^{(2)}\right)}\right\}_{k=1}^{\infty}$ of $\left\{x_{2}^{\left(L, n_{k}^{(1)}\right)}\right\}_{k=1}^{\infty}$ that weakly converges to some $x_{2}^{(L, 0)} \in L^{2}[0,1]$. By induction, there exists a subsequence $\left\{x_{j}^{\left(L, n_{k}^{(j)}\right)}\right\}_{k=1}^{\infty}$ of $\left\{x_{j}^{\left(L, n_{k}^{(j-1)}\right)}\right\}_{k=1}^{\infty}$ that weakly converges to some $x_{j}^{(L, 0)} \in L^{2}[0,1]$ for $j=1, \cdots, q$. The above argument can also apply to the sequences $\left\{x_{j}^{(U, n)}\right\}_{n=1}^{\infty}$ for $j=1, \cdots, q$. Therefore we can construct two subsequences $\left\{\mathbf{x}^{\left(L, n_{k}\right)}\right\}_{k=1}^{\infty}$ and $\left\{\mathbf{x}^{\left(U, n_{k}\right)}\right\}_{k=1}^{\infty}$ that weakly converges to $\mathbf{x}^{(L, 0)}$ and $\mathbf{x}^{(U, 0)}$, respectively. Since $\mathbf{x}^{\left(L, n_{k}\right)}$ and $\mathbf{x}^{\left(U, n_{k}\right)}$ are feasible solutions of problem SOP by Proposition 6 , for $i=1, \cdots, p$, $j=1, \cdots, q$ and $t \in[0,1]$, we have

$$
\begin{aligned}
& \sum_{\left\{j: j \in P_{i}\right\}} b_{i j}^{L}(t) \cdot x_{j}^{\left(L, n_{k}\right)}(t)+\sum_{\left\{j: j \in N_{i}\right\}} b_{i j}^{L}(t) \cdot x_{j}^{\left(U, n_{k}\right)}(t) \leq c_{i}^{L}(t) ; \\
& \sum_{\left\{j: j \in N_{i}\right\}} b_{i j}^{U}(t) \cdot x_{j}^{\left(L, n_{k}\right)}(t)+\sum_{\left\{j: j \in P_{i}\right\}} b_{i j}^{U}(t) \cdot x_{j}^{\left(U, n_{k}\right)}(t) \leq c_{i}^{U}(t) ; \\
& x_{j}^{\left(U, n_{k}\right)}(t) \geq x_{j}^{\left(L, n_{k}\right)}(t) \geq 0 ; \\
& \text { each } x_{j}^{\left(L, n_{k}\right)}(t) \text { is an increasing function on }[0,1] ; \\
& \text { each } x_{j}^{\left(U, n_{k}\right)}(t) \text { is a decreasing function on }[0,1] .
\end{aligned}
$$

Using Lemma 9, we see that the sequences

$$
\left\{\sum_{\left\{j: j \in P_{i}\right\}} b_{i j}^{L}(t) \cdot x_{j}^{\left(L, n_{k}\right)}(t)+\sum_{\left\{j: j \in N_{i}\right\}} b_{i j}^{L}(t) \cdot x_{j}^{\left(U, n_{k}\right)}(t)\right\}_{k=1}^{\infty}
$$

and

$$
\left\{\sum_{\left\{j: j \in N_{i}\right\}} b_{i j}^{U}(t) \cdot x_{j}^{\left(L, n_{k}\right)}(t)+\sum_{\left\{j: j \in P_{i}\right\}} b_{i j}^{U}(t) \cdot x_{j}^{\left(U, n_{k}\right)}(t)\right\}_{k=1}^{\infty}
$$

weakly converge to

$$
\sum_{\left\{j: j \in P_{i}\right\}} b_{i j}^{L}(t) \cdot x_{j}^{(L, 0)}(t)+\sum_{\left\{j: j \in N_{i}\right\}} b_{i j}^{L}(t) \cdot x_{j}^{(U, 0)}(t)
$$

and

$$
\sum_{\left\{j: j \in N_{i}\right\}} b_{i j}^{U}(t) \cdot x_{j}^{(L, 0)}(t)+\sum_{\left\{j: j \in P_{i}\right\}} b_{i j}^{U}(t) \cdot x_{j}^{(U, 0)}(t),
$$

respectively, which imply, by using (158), (159) and Lemma 8,

$$
\begin{aligned}
& \sum_{\left\{j: j \in P_{i}\right\}} b_{i j}^{L}(t) \cdot x_{j}^{(L, 0)}(t)+\sum_{\left\{j: j \in N_{i}\right\}} b_{i j}^{L}(t) \cdot x_{j}^{(U, 0)}(t) \\
& \leq \limsup _{k \rightarrow \infty}\left[\sum_{\left\{j: j \in P_{i}\right\}} b_{i j}^{L}(t) \cdot x_{j}^{\left(L, n_{k}\right)}(t)+\sum_{\left\{j: j \in N_{i}\right\}} b_{i j}^{L}(t) \cdot x_{j}^{\left(U, n_{k}\right)}(t)\right] \leq c_{i}^{L}(t) \text { a.e. on }[0,1]
\end{aligned}
$$


and

$$
\begin{aligned}
& \sum_{\left\{j: j \in N_{i}\right\}} b_{i j}^{U}(t) \cdot x_{j}^{(L, 0)}(t)+\sum_{\left\{j: j \in P_{i}\right\}} b_{i j}^{U}(t) \cdot x_{j}^{(U, 0)}(t) \\
& \quad \leq \limsup _{k \rightarrow \infty}\left[\sum_{\left\{j: j \in N_{i}\right\}} b_{i j}^{U}(t) \cdot x_{j}^{\left(L, n_{k}\right)}(t)+\sum_{\left\{j: j \in P_{i}\right\}} b_{i j}^{U}(t) \cdot x_{j}^{\left(U, n_{k}\right)}(t)\right] \leq c_{i}^{U}(t) \text { a.e. on }[0,1] .
\end{aligned}
$$

Using (160), (155) and Lemma 8, we have

$$
x_{j}^{(U, 0)}(t) \geq \liminf _{k \rightarrow \infty} x_{j}^{\left(U, n_{k}\right)}(t) \geq \limsup _{k \rightarrow \infty} x_{j}^{\left(L, n_{k}\right)}(t) \geq x_{j}^{(L, 0)}(t) \text { a.e. on }[0,1] .
$$

From Lemma 8, we also have

$$
0 \leq \liminf _{k \rightarrow \infty} x_{j}^{\left(L, n_{k}\right)}(t) \leq x_{j}^{(L, 0)}(t) \leq \limsup _{k \rightarrow \infty} x_{j}^{\left(L, n_{k}\right)}(t) \text { a.e. on }[0,1]
$$

and

$$
0 \leq \liminf _{k \rightarrow \infty} x_{j}^{\left(U, n_{k}\right)}(t) \leq x_{j}^{(U, 0)}(t) \leq \limsup _{k \rightarrow \infty} x_{j}^{\left(U, n_{k}\right)}(t) \text { a.e. on }[0,1]
$$

Let $\mathcal{N}$ be a subset of $[0,1]$ such that the inequalities (163)-(167) are violated. Then $\mathcal{N}$ has measure zero. In other words, if $t \in[0,1] \backslash \mathcal{N}$, then all the inequalities (163)-(167) are satisfied. Now, for $j=1, \cdots, q$, we define the real-valued functions $x_{j}^{(* L)}$ and $x_{j}^{(* U)}$ on $[0,1]$ as follows

$$
x_{j}^{(* L)}(t)= \begin{cases}\liminf _{k \rightarrow \infty} x_{j}^{\left(L, n_{k}\right)}(t) & \text { if } t \in \mathcal{N} \\ x_{j}^{(L, 0)}(t) & \text { if } t \in[0,1] \backslash \mathcal{N}\end{cases}
$$

and

$$
x_{j}^{(* U)}(t)= \begin{cases}\liminf _{k \rightarrow \infty} x_{j}^{\left(U, n_{k}\right)}(t) & \text { if } t \in \mathcal{N} \\ x_{j}^{(U, 0)}(t) & \text { if } t \in[0,1] \backslash \mathcal{N}\end{cases}
$$

Since the set $\mathcal{N}$ has measure zero, it is clear to see that

$$
x_{j}^{(* L)}(t)=x_{j}^{(L, 0)}(t) \text { and } x_{j}^{(* U)}(t)=x_{j}^{(U, 0)}(t) \text { a.e. on }[0,1] .
$$

We are going to show that $\mathbf{x}^{*}(t)=\left(\mathbf{x}^{(* L)}, \mathbf{x}^{(* U)}\right)$ formed from (168) is a feasible solution of problem SOP.

For $t \in[0,1] \backslash \mathcal{N}$, from (163)-(165), we see that $\mathbf{x}^{*}(t)$ satisfies the inequalities (10)-(12). For $t \in \mathcal{N}$, by referring to (119)-(121), we also see that $\mathbf{x}^{*}(t)$ satisfies the inequalities (10)-(12). We remain to show that $x_{j}^{(* L)}$ is an increasing function on $[0,1]$ and $x_{j}^{(* U)}$ is a decreasing function on $[0,1]$ for $j=1, \cdots, q$. For $t_{1}<t_{2}$, from (161) and (162), we have

$$
x_{j}^{\left(L, n_{k}\right)}\left(t_{1}\right) \leq x_{j}^{\left(L, n_{k}\right)}\left(t_{2}\right) \text { and } x_{j}^{\left(U, n_{k}\right)}\left(t_{1}\right) \geq x_{j}^{\left(U, n_{k}\right)}\left(t_{2}\right) .
$$

Now we consider the following cases. 
- $\quad$ Suppose that $t_{1}, t_{2} \in[0,1] \backslash \mathcal{N}$. Then we have

$$
\begin{aligned}
x_{j}^{(* L)}\left(t_{1}\right) & =x_{j}^{(L, 0)}\left(t_{1}\right) \leq \underset{k \rightarrow \infty}{\limsup } x_{j}^{\left(L, n_{k}\right)}\left(t_{1}\right)(\text { by }(166)) \\
& \leq \liminf _{k \rightarrow \infty} x_{j}^{\left(L, n_{k}\right)}\left(t_{2}\right)(\text { by }(156)) \\
& \leq x_{j}^{(L, 0)}\left(t_{2}\right)(\text { by }(166)) \\
& =x_{j}^{(* L)}\left(t_{2}\right) .
\end{aligned}
$$

We also have

$$
\begin{aligned}
x_{j}^{(* U)}\left(t_{1}\right) & =x_{j}^{(U, 0)}\left(t_{1}\right) \geq \liminf _{k \rightarrow \infty} x_{j}^{\left(U, n_{k}\right)}\left(t_{1}\right)(\text { by }(167)) \\
& \geq \limsup _{k \rightarrow \infty} x_{j}^{\left(U, n_{k}\right)}\left(t_{2}\right)(\text { by }(157)) \\
& \geq x_{j}^{(U, 0)}\left(t_{2}\right)(\text { by }(167)) \\
& =x_{j}^{(* U)}\left(t_{2}\right) .
\end{aligned}
$$

- Suppose that $t_{1} \in \mathcal{N}$ and $t_{2} \in[0,1] \backslash \mathcal{N}$. Then we have

$$
\begin{aligned}
x_{j}^{(* L)}\left(t_{1}\right) & =\liminf _{k \rightarrow \infty} x_{j}^{\left(L, n_{k}\right)}\left(t_{1}\right) \leq \liminf _{k \rightarrow \infty} x_{j}^{\left(L, n_{k}\right)}\left(t_{2}\right)(\text { by }(169)) \\
& \leq x_{j}^{(L, 0)}\left(t_{2}\right)(\text { by }(166)) \\
& =x_{j}^{(* L)}\left(t_{2}\right) .
\end{aligned}
$$

We also have

$$
\begin{aligned}
x_{j}^{(* U)}\left(t_{1}\right) & =\liminf _{k \rightarrow \infty} x_{j}^{\left(U, n_{k}\right)}\left(t_{1}\right) \geq \limsup _{k \rightarrow \infty} x_{j}^{\left(U, n_{k}\right)}\left(t_{2}\right)(\text { by }(157)) \\
& \geq x_{j}^{(U, 0)}\left(t_{2}\right)(\text { by }(166)) \\
& =x_{j}^{(* U)}\left(t_{2}\right) .
\end{aligned}
$$

- Suppose that $t_{1} \in[0,1] \backslash \mathcal{N}$ and $t_{2} \in \mathcal{N}$. Then we have

$$
\begin{aligned}
x_{j}^{(* L)}\left(t_{1}\right) & =x_{j}^{(L, 0)}\left(t_{1}\right) \leq \underset{k \rightarrow \infty}{\limsup } x_{j}^{\left(L, n_{k}\right)}\left(t_{1}\right)(\text { by }(166)) \\
& \leq \liminf _{k \rightarrow \infty} x_{j}^{\left(L, n_{k}\right)}\left(t_{2}\right)(\text { by }(156)) \\
& =x_{j}^{(* L)}\left(t_{2}\right) .
\end{aligned}
$$

We also have

$$
\begin{aligned}
x_{j}^{(* U)}\left(t_{1}\right) & =x_{j}^{(U, 0)}\left(t_{1}\right) \geq \liminf _{k \rightarrow \infty} x_{j}^{\left(U, n_{k}\right)}\left(t_{1}\right)(\text { by }(167)) \\
& \geq \liminf _{k \rightarrow \infty} x_{j}^{\left(U, n_{k}\right)}\left(t_{2}\right)(\text { by }(169)) \\
& =x_{j}^{(* U)}\left(t_{2}\right) .
\end{aligned}
$$


- $\quad$ Suppose that $t_{1}, t_{2} \in \mathcal{N}$. Then we have

$$
\begin{aligned}
x_{j}^{(* L)}\left(t_{1}\right) & =\liminf _{k \rightarrow \infty} x_{j}^{\left(L, n_{k}\right)}\left(t_{1}\right) \leq \liminf _{k \rightarrow \infty} x_{j}^{\left(L, n_{k}\right)}\left(t_{2}\right)(\text { by }(169)) \\
& =x_{j}^{(* L)}\left(t_{2}\right) .
\end{aligned}
$$

We also have

$$
\begin{aligned}
x_{j}^{(* U)}\left(t_{1}\right) & =\liminf _{k \rightarrow \infty} x_{j}^{\left(U, n_{k}\right)}\left(t_{1}\right) \geq \liminf _{k \rightarrow \infty} x_{j}^{\left(U, n_{k}\right)}\left(t_{2}\right)(\text { by }(169)) \\
& =x_{j}^{(* U)}\left(t_{2}\right) .
\end{aligned}
$$

Therefore we conclude that $\left(\mathbf{x}^{(* L)}, \mathbf{x}^{(* U)}\right)$ satisfies (13) and (14). This shows that $\mathbf{x}^{*}=\left(\mathbf{x}^{(* L)}, \mathbf{x}^{(* U)}\right)$ is indeed a feasible solution of problem SOP.

Next we want to prove the optimality. Given any feasible solution $\left(\mathbf{x}^{L}, \mathbf{x}^{U}\right)$ of problem SOP, by referring to (150), we can also obtain

$$
\begin{aligned}
\sum_{j \in P}\left(\int_{0}^{1} a_{j}^{L}(t) \cdot x_{j}^{\left(L, n_{k}\right)}(t) d \alpha+\int_{0}^{1} a_{j}^{U}(t) \cdot x_{j}^{\left(U, n_{k}\right)}(t) d \alpha\right) \\
\quad+\sum_{j \in N}\left(\int_{0}^{1} a_{j}^{L}(t) \cdot x_{j}^{\left(U, n_{k}\right)}(t) d \alpha+\int_{0}^{1} a_{j}^{U}(t) \cdot x_{j}^{\left(L, n_{k}\right)}(t) d \alpha\right) \\
\geq \sum_{j \in P}\left(\int_{0}^{1} a_{j}^{L}(t) \cdot x_{j}^{L}(t) d \alpha+\int_{0}^{1} a_{j}^{U}(t) \cdot x_{j}^{U}(t) d \alpha\right) \\
\quad+\sum_{j \in N}\left(\int_{0}^{1} a_{j}^{L}(t) \cdot x_{j}^{U}(t) d \alpha+\int_{0}^{1} a_{j}^{U}(t) \cdot x_{j}^{L}(t) d \alpha\right) \\
+\mathfrak{a}^{\left(P L, n_{k}\right)}+\mathfrak{a}^{\left(N U, n_{k}\right)}+\mathfrak{a}^{\left(N L, n_{k}\right)}+\mathfrak{a}^{\left(P U, n_{k}\right)}-\mathfrak{c}^{\left(L, n_{k}\right)}-\mathfrak{c}^{\left(U, n_{k}\right)},
\end{aligned}
$$

where

$$
\mathfrak{a}^{\left(P L, n_{k}\right)} \rightarrow 0, \quad \mathfrak{a}^{\left(N U, n_{k}\right)} \rightarrow 0, \quad \mathfrak{a}^{\left(N L, n_{k}\right)} \rightarrow 0 \text { and } \mathfrak{a}^{\left(P U, n_{k}\right)} \rightarrow 0 \text { as } k \rightarrow \infty .
$$

and

$$
\mathfrak{c}^{\left(L, n_{k}\right)} \rightarrow 0 \text { and } \mathfrak{c}^{\left(U, n_{k}\right)} \rightarrow 0 \text { as } k \rightarrow \infty .
$$

by referring to (136)-(139) and (147), respectively. Since the subsequences $\left\{x_{j}^{\left(L, n_{k}\right)}\right\}_{k=1}^{\infty}$ and $\left\{x_{j}^{\left(U, n_{k}\right)}\right\}_{k=1}^{\infty}$ are weakly convergent to $x_{j}^{(L, 0)}$ and $x_{j}^{(U, 0)}$, respectively, for $j=1, \cdots, q$, we have

$$
\begin{aligned}
\lim _{k \rightarrow \infty} \sum_{j \in P} \int_{0}^{1} a_{j}^{L}(t) \cdot x_{j}^{\left(L, n_{k}\right)}(t) d t & =\sum_{j \in P} \int_{0}^{1} a_{j}^{L}(t) \cdot x_{j}^{(L, 0)}(t) d t \text { (using the weak convergence) } \\
& =\sum_{j \in P} \int_{0}^{1} a_{j}^{L}(t) \cdot x_{j}^{(* L)}(t) d t \text { (by (168)) } \\
\lim _{k \rightarrow \infty} \sum_{j \in N} \int_{0}^{1} a_{j}^{L}(t) \cdot x_{j}^{\left(U, n_{k}\right)}(t) d t & =\sum_{j \in N} \int_{0}^{1} a_{j}^{L}(t) \cdot x_{j}^{(U, 0)}(t) d t=\sum_{j \in N} \int_{0}^{1} a_{j}^{L}(t) \cdot x_{j}^{(* U)}(t) d t . \\
\lim _{k \rightarrow \infty} \sum_{j \in N} \int_{0}^{1} a_{j}^{U}(t) \cdot x_{j}^{\left(L, n_{k}\right)}(t) d t & =\sum_{j \in N} \int_{0}^{1} a_{j}^{U}(t) \cdot x_{j}^{(L, 0)}(t) d t=\sum_{j \in N} \int_{0}^{1} a_{j}^{U}(t) \cdot x_{j}^{(* L)}(t) d t . \\
\lim _{k \rightarrow \infty} \sum_{j \in P} \int_{0}^{1} a_{j}^{U}(t) \cdot x_{j}^{\left(U, n_{k}\right)}(t) d t & =\sum_{j \in P} \int_{0}^{1} a_{j}^{U}(t) \cdot x_{j}^{(U, 0)}(t) d t=\sum_{j \in P} \int_{0}^{1} a_{j}^{U}(t) \cdot x_{j}^{(* U)}(t) d t .
\end{aligned}
$$


By taking limit on both sides of (170) and using (171)-(176), we obtain

$$
\begin{aligned}
& \sum_{j \in P}\left(\int_{0}^{1} a_{j}^{L}(t) \cdot x_{j}^{(* L)}(t) d \alpha+\int_{0}^{1} a_{j}^{U}(t) \cdot x_{j}^{(* U)}(t) d \alpha\right) \\
& \quad+\sum_{j \in N}\left(\int_{0}^{1} a_{j}^{L}(t) \cdot x_{j}^{(* U)}(t) d \alpha+\int_{0}^{1} a_{j}^{U}(t) \cdot x_{j}^{(* L)}(t) d \alpha\right) \\
& \geq \sum_{j \in P}\left(\int_{0}^{1} a_{j}^{L}(t) \cdot x_{j}^{L}(t) d \alpha+\int_{0}^{1} a_{j}^{U}(t) \cdot x_{j}^{U}(t) d \alpha\right) \\
& \quad+\sum_{j \in N}\left(\int_{0}^{1} a_{j}^{L}(t) \cdot x_{j}^{U}(t) d \alpha+\int_{0}^{1} a_{j}^{U}(t) \cdot x_{j}^{L}(t) d \alpha\right) .
\end{aligned}
$$

Since $\left(\mathbf{x}^{L}, \mathbf{x}^{U}\right)$ can be any feasible solution of problem SOP, it shows that $\left(\mathbf{x}^{(* L)}, \mathbf{x}^{(* U)}\right)$ is an optimal solution of problem SOP. This completes the proof.

\section{Tighter Bound of Error Estimation for Nonnegative Constraints}

Although the error estimation $\varepsilon_{n}$ presented in (106) can be used to solve the fuzzy linear programming problem FLP in which all of the fuzzy numbers in the constraints are assumed to be nonnegative, in this section, we shall plan to derive the tighter bound of error estimation when all of the fuzzy numbers in the constraints are taken to be nonnegative. The key issue is that the real-valued functions $y_{i}^{(I, n)}, y_{i}^{(I I, n)}, y_{j}^{(I I I, n)}, y_{j}^{(I V, n)}$ and $y_{j}^{(V, n)}$ for $i=1, \cdots, p$ and $j=1, \cdots, q$ do not need to be defined in the forms of (88)-(92). More simple forms can be used for the nonnegative problem. We need to remark that these simple forms will not be the special case of the forms presented in (88)-(92). Therefore we need to separately study the nonnegative problem FLP in this section.

We first note that, for the nonnegative case, the index sets $N_{i}=\varnothing$ for all $i=1, \cdots, p$. Therefore, all of the expressions involving the index sets $N_{i}$ for $i=1, \cdots, p$ can be re-written as the simple forms. Since the real-valued functions presented in (88)-(92) do not involve the index sets $N_{i}$. It means that those real-valued functions cannot be re-written as the simple forms. Therefore we are going to present the different forms for the problem FLP with nonnegative constraints, which means that, as we mentioned above, the problem FLP with nonnegative constraints should be separately studied.

Now the problem FLP with nonnegative constraints, denoted by (NFLP), can be re-written from FLP by taking $N_{i}=\varnothing$ for all $i=1, \cdots, p$, which is shown below:

$(\mathrm{NFLP}) \quad \max \quad\left(\widetilde{a}_{1} \otimes \widetilde{x}_{1}\right) \oplus\left(\widetilde{a}_{2} \otimes \widetilde{x}_{2}\right) \oplus \cdots \oplus\left(\widetilde{a}_{q} \otimes \widetilde{x}_{q}\right)$

subject to $\widetilde{b}_{i 1 \alpha}^{L} \cdot \widetilde{x}_{1 \alpha}^{L}+\widetilde{b}_{i 2 \alpha}^{L} \cdot \widetilde{x}_{2 \alpha}^{L}+\cdots+\widetilde{b}_{i q \alpha}^{L} \cdot \widetilde{x}_{q \alpha}^{L} \leq \widetilde{c}_{i \alpha}^{L}$ for $i=1, \cdots, p$ and $\alpha \in[0,1]$;

$\widetilde{b}_{i 1 \alpha}^{U} \cdot \widetilde{x}_{1 \alpha}^{U}+\widetilde{b}_{i 2 \alpha}^{U} \cdot \widetilde{x}_{2 \alpha}^{U}+\cdots+\widetilde{b}_{i q \alpha}^{U} \cdot \widetilde{x}_{q \alpha}^{U} \leq \widetilde{c}_{i \alpha}^{U}$ for $i=1, \cdots, p$ and $\alpha \in[0,1]$;

$\widetilde{x}_{j \alpha}^{U} \geq \widetilde{x}_{j \alpha}^{L} \geq 0$ for $j=1, \cdots, q$ and $\alpha \in[0,1]$;

each $\widetilde{x}_{j \alpha}^{L}$ is an increasing function with respect to $\alpha$ on $[0,1]$ for $j=1, \cdots, q$;

each $\widetilde{x}_{j \alpha}^{U}$ is a decreasing function with respect to $\alpha$ on $[0,1]$ for $j=1, \cdots, q$. 
Also its corresponding scalar optimization problem NSOP can be written as follows:

$$
\begin{aligned}
& (\mathrm{NSOP}) \quad \max \quad \sum_{j \in P}\left(\int_{0}^{1} a_{j}^{L}(t) \cdot x_{j}^{L}(t) d \alpha+\int_{0}^{1} a_{j}^{U}(t) \cdot x_{j}^{U}(t) d \alpha\right) \\
& +\sum_{j \in N}\left(\int_{0}^{1} a_{j}^{L}(t) \cdot x_{j}^{U}(t) d \alpha+\int_{0}^{1} a_{j}^{U}(t) \cdot x_{j}^{L}(t) d \alpha\right) \\
& \text { subject to } \sum_{j=1}^{q} b_{i j}^{L}(t) \cdot x_{j}^{L}(t) \leq c_{i}^{L}(t) \text { for all } t \in[0,1] \text { and } i=1, \cdots, p \text {; } \\
& \sum_{j=1}^{q} b_{i j}^{U}(t) \cdot x_{j}^{U}(t) \leq c_{i}^{U}(t) \text { for all } t \in[0,1] \text { and } i=1, \cdots, p ; \\
& x_{j}^{U}(t) \geq x_{j}^{L}(t) \geq 0 \text { for } j=1, \cdots, q \text { and } t \in[0,1] ; \\
& \text { each } x_{j}^{L}(t) \text { is an increasing function on }[0,1] \text { for } j=1, \cdots, q \text {; } \\
& \text { each } x_{j}^{U}(t) \text { is a decreasing function on }[0,1] \text { for } j=1, \cdots, q \text {. }
\end{aligned}
$$

In this case, given any fixed $\delta$ with $0<\delta<1$, the auxiliary problem is given by

$$
\begin{aligned}
\left(\operatorname{NSOP}_{\delta}\right) \quad \max \quad & \sum_{j \in P}\left(\int_{0}^{1} a_{j}^{L}(t) \cdot x_{j}^{L}(t) d \alpha+\int_{0}^{1} a_{j}^{U}(t) \cdot x_{j}^{U}(t) d \alpha\right) \\
& +\sum_{j \in N}\left(\int_{0}^{1} a_{j}^{L}(t) \cdot x_{j}^{U}(t) d \alpha+\int_{0}^{1} a_{j}^{U}(t) \cdot x_{j}^{L}(t) d \alpha\right) \\
\text { subject to } \quad & \sum_{j=1}^{q} b_{i j}^{L}(t) \cdot x_{j}^{L}(t) \leq c_{i}^{L}(t) \text { for all } t \in[0,1] \text { and } i=1, \cdots, p ; \\
& \sum_{j=1}^{q} b_{i j}^{U}(t) \cdot x_{j}^{U}(t) \leq c_{i}^{U}(t) \text { for all } t \in[0,1] \text { and } i=1, \cdots, p ; \\
& x_{j}^{U}(t) \geq x_{j}^{L}(t) \geq 0 \text { for } j=1, \cdots, q \text { and } t \in[0,1] ; \\
& x_{j}^{L}(t) \leq x_{j}^{L}(t+\delta) \text { for all } t \in[0,1-\delta] \text { and } j=1, \cdots, q ; \\
& x_{j}^{U}(t) \geq x_{j}^{U}(t+\delta) \text { for all } t \in[0,1-\delta] \text { and } j=1, \cdots, q .
\end{aligned}
$$

For $\delta=1 / n$, the dual problem of $\left(\mathrm{NSOP}_{n}\right)$ is also given by

$$
\left(\mathrm{DNSOP}_{n}\right) \quad \min \sum_{i=1}^{p} \int_{0}^{1} c_{i}^{L}(t) \cdot y_{i}^{I}(t) d t+\sum_{i=1}^{p} \int_{0}^{1} c_{i}^{U}(t) \cdot y_{i}^{I I}(t) d t
$$

subject to $\sum_{i=1}^{p} b_{i j}^{L}(t) \cdot y_{i}^{I}(t)+y_{j}^{I I I}(t)+y_{j}^{I V}(t) \geq a_{j}^{L}(t)$

$$
\text { for all } t \in F_{1}^{(n)}=[0, \delta)=[0,1 / n) \text { and } j \in P \text {; }
$$

$$
\begin{aligned}
& \sum_{i=1}^{p} b_{i j}^{L}(t) \cdot y_{i}^{I}(t)+y_{j}^{I I I}(t)+y_{j}^{I V}(t) \geq a_{j}^{U}(t) \text { for all } t \in F_{1}^{(n)} \text { and } j \in N \\
& \sum_{i=1}^{p} b_{i j}^{U}(t) \cdot y_{i}^{I I}(t)-y_{j}^{I I I}(t)-y_{j}^{V}(t) \geq a_{j}^{L}(t) \text { for all } t \in F_{1}^{(n)} \text { and } j \in N
\end{aligned}
$$




$$
\begin{aligned}
& \sum_{i=1}^{p} b_{i j}^{U}(t) \cdot y_{i}^{I I}(t)-y_{j}^{I I I}(t)-y_{j}^{V}(t) \geq a_{j}^{U}(t) \text { for all } t \in F_{1}^{(n)} \text { and } j \in P \\
& \sum_{i=1}^{p} b_{i j}^{L}(t) \cdot y_{i}^{I}(t)+y_{j}^{I I I}(t)+y_{j}^{I V}(t)-y_{j}^{I V}\left(t-\frac{1}{n}\right) \geq a_{j}^{L}(t) \\
& \text { for all } t \in F_{l}^{(n)}, l=2, \cdots, n-1 \text { and } j \in P \text {; } \\
& \sum_{i=1}^{p} b_{i j}^{L}(t) \cdot y_{i}^{I}(t)+y_{j}^{I I I}(t)+y_{j}^{I V}(t)-y_{j}^{I V}\left(t-\frac{1}{n}\right) \geq a_{j}^{U}(t) \\
& \text { for all } t \in F_{l}^{(n)}, l=2, \cdots, n-1 \text { and } j \in N \text {; } \\
& \sum_{i=1}^{p} b_{i j}^{U}(t) \cdot y_{i}^{I I}(t)-y_{j}^{I I I}(t)-y_{j}^{V}(t)+y_{j}^{V}\left(t-\frac{1}{n}\right) \geq a_{j}^{L}(t) \\
& \text { for all } t \in F_{l}^{(n)}, l=2, \cdots, n-1 \text { and } j \in N \text {; } \\
& \sum_{i=1}^{p} b_{i j}^{U}(t) \cdot y_{i}^{I I}(t)-y_{j}^{I I I}(t)-y_{j}^{V}(t)+y_{j}^{V}\left(t-\frac{1}{n}\right) \geq a_{j}^{U}(t) \\
& \text { for all } t \in F_{l}^{(n)}, l=2, \cdots, n-1 \text { and } j \in P \text {; } \\
& \sum_{i=1}^{p} b_{i j}^{L}(t) \cdot y_{i}^{I}(t)+y_{j}^{I I I}(t)-y_{j}^{I V}\left(t-\frac{1}{n}\right) \geq a_{j}^{L}(t) \\
& \text { for all } t \in F_{n}^{(n)} \cup\{1\}=[1-\delta, 1]=[1-1 / n, 1] \text { and } j \in P ; \\
& \sum_{i=1}^{p} b_{i j}^{L}(t) \cdot y_{i}^{I}(t)+y_{j}^{I I I}(t)-y_{j}^{I V}\left(t-\frac{1}{n}\right) \geq a_{j}^{U}(t) \\
& \text { for all } t \in F_{n}^{(n)} \cup\{1\}=\bar{E}_{n}^{(n)} \text { and } j \in N \text {; } \\
& \sum_{i=1}^{p} b_{i j}^{U}(t) \cdot y_{i}^{I I}(t)-y_{j}^{I I I}(t)+y_{j}^{V}\left(t-\frac{1}{n}\right) \geq a_{j}^{L}(t) \\
& \text { for all } t \in F_{n}^{(n)} \cup\{1\} \text { and } j \in N \text {; } \\
& \sum_{i=1}^{p} b_{i j}^{U}(t) \cdot y_{i}^{I I}(t)-y_{j}^{I I I}(t)+y_{j}^{V}\left(t-\frac{1}{n}\right) \geq a_{j}^{U}(t) \\
& \text { for all } t \in F_{n}^{(n)} \cup\{1\} \text { and } j \in P \text {; } \\
& y_{i}^{I}(t), y_{i}^{I I}(t) \geq 0 \text { for all } t \in[0,1] \text { and } i=1, \cdots, p ; \\
& y_{j}^{I I I}(t), y_{j}^{I V}(t), y_{j}^{V}(t) \geq 0 \text { for all } t \in[0,1] \text { and } j=1, \cdots, q \text {. }
\end{aligned}
$$


Regarding the discretization problem, the primal linear programming problem is given below

$$
\begin{aligned}
\left(\mathrm{NP}_{n}\right) \quad \max \quad \sum_{j \in P} & \left(\sum_{l=1}^{n} \frac{1}{n} \cdot a_{l j}^{(L, n)} \cdot z_{l j}^{L}+\sum_{l=1}^{n} \frac{1}{n} \cdot a_{l j}^{(U, n)} \cdot z_{l j}^{U}\right) \\
& \quad+\sum_{j \in N}\left(\sum_{l=1}^{n} \frac{1}{n} \cdot a_{l j}^{(U, n)} \cdot z_{l j}^{L}+\sum_{l=1}^{n} \frac{1}{n} \cdot a_{l j}^{(L, n)} \cdot z_{l j}^{U}\right) \\
\text { subject to } \quad \sum_{j=1}^{q} b_{l i j}^{(L, n)} z_{l j}^{L} \leq c_{l i}^{(L, n)} \text { for } i=1, \cdots, p \text { and } l=1, \cdots, n ; & \\
& \sum_{j=1}^{q} b_{l i j}^{(U, n)} z_{l j}^{U} \leq c_{l i}^{(U, n)} \text { for } i=1, \cdots, p \text { and } l=1, \cdots, n ; \\
& z_{l j}^{U} \geq z_{l j}^{L} \text { for } j=1, \cdots, q \text { and } l=1, \cdots, n ; \\
& z_{l j}^{L} \leq z_{l+1, j}^{L} \text { for } j=1, \cdots, q \text { and } l=1, \cdots, n-1 ; \\
& z_{l j}^{U} \geq z_{l+1, j}^{U} \text { for } j=1, \cdots, q \text { and } l=1, \cdots, n-1 ; \\
& z_{l j}^{L} \geq 0 \text { and } z_{l j}^{U} \geq 0 \text { for } j=1, \cdots, q \text { and } l=1, \cdots, n,
\end{aligned}
$$

and the dual linear programming problem is given below

$$
\begin{gathered}
\left(\mathrm{ND}_{n}\right) \quad \min \quad \sum_{i=1}^{p} \sum_{l=1}^{n} \frac{1}{n} \cdot c_{l i}^{(L, n)} \cdot w_{l i}^{I}+\sum_{i=1}^{p} \sum_{l=1}^{n} \frac{1}{n} \cdot c_{l i}^{(U, n)} \cdot w_{l i}^{I I} \\
\text { subject to } \sum_{i=1}^{p} b_{1 i j}^{(L, n)} \cdot w_{1 i}^{I}+w_{1 j}^{I I I}+w_{1 j}^{I V} \geq a_{1 j}^{(L, n)} \text { for } j \in P ; \\
\\
\sum_{i=1}^{p} b_{1 i j}^{(L, n)} \cdot w_{1 i}^{I}+w_{1 j}^{I I I}+w_{1 j}^{I V} \geq a_{1 j}^{(U, n)} \text { for } j \in N ; \\
\sum_{i=1}^{p} b_{1 i j}^{(U, n)} \cdot w_{1 i}^{I I}-w_{1 j}^{I I I}-w_{1 j}^{V} \geq a_{1 j}^{(L, n)} \text { for } j \in N ; \\
\sum_{i=1}^{p} b_{1 i j}^{(U, n)} \cdot w_{1 i}^{I I}-w_{1 j}^{I I I}-w_{1 j}^{V} \geq a_{1 j}^{(U, n)} \text { for } j \in P ; \\
\sum_{i=1}^{p} b_{l i j}^{(L, n)} \cdot w_{l i}^{I}+w_{l j}^{I I I}+w_{l j}^{I V}-w_{l-1, j}^{I V} \geq a_{l j}^{(L, n)} \\
\quad \text { for } j \in P \text { and } l=2, \cdots, n-1 ; \\
\sum_{i=1}^{p} b_{l i j}^{(L, n)} \cdot w_{l i}^{I}+w_{l j}^{I I I}+w_{l j}^{I V}-w_{l-1, j}^{I V} \geq a_{l j}^{(U, n)} \\
\text { for } j \in N \text { and } l=2, \cdots, n-1 ;
\end{gathered}
$$




$$
\begin{aligned}
& \sum_{i=1}^{p} b_{l i j}^{(U, n)} \cdot w_{l i}^{I I}-w_{l j}^{I I I}-w_{l j}^{V}+w_{l-1, j}^{V} \geq a_{l j}^{(L, n)} \\
& \text { for } j \in N \text { and } l=2, \cdots, n-1 ; \\
& \sum_{i=1}^{p} b_{l i j}^{(U, n)} \cdot w_{l i}^{I I}-w_{l j}^{I I I}-w_{l j}^{V}+w_{l-1, j}^{V} \geq a_{l j}^{(U, n)} \\
& \quad \text { for } j \in P \text { and } l=2, \cdots, n-1 ; \\
& \sum_{i=1}^{p} b_{n i j}^{(L, n)} \cdot w_{n i}^{I I}+w_{n j}^{I I I}-w_{n-1, j}^{I V} \geq a_{n j}^{(L, n)} \text { for } j \in P ; \\
& \sum_{i=1}^{p} b_{n i j}^{(L, n)} \cdot w_{n i}^{I}+w_{n j}^{I I I}-w_{n-1, j}^{I V} \geq a_{n j}^{(U, n)} \text { for } j \in N ; \\
& \sum_{i=1}^{p} b_{n i j}^{(U, n)} \cdot w_{n i}^{I I}-w_{n j}^{I I I}+w_{n-1, j}^{V} \geq a_{n j}^{(U, n)} \text { for } j \in P ; \\
& \sum_{i=1}^{p} b_{n i j}^{(U, n)} \cdot w_{n i}^{I I}-w_{n j}^{I I I}+w_{n-1, j}^{V} \geq a_{n j}^{(L, n)} \text { for } j \in N ;
\end{aligned}
$$

For further discussion, we first define some notations. Let $\mathbf{w}^{(* n)}$ be an optimal solution of problem $\left(\mathrm{ND}_{n}\right)$. For $j=1, \cdots, q$, we define the real-valued functions $h_{l j}^{(L, n)}$ and $h_{l j}^{(U, n)}$ on $F_{l}^{(n)}$ for $l=1, \cdots, n-1$ and $h_{n j}^{(L, n)}$ and $h_{n j}^{(U, n)}$ on $F_{n}^{(n)} \cup\{1\}=\bar{E}_{n}^{(n)}$ by

$$
h_{l j}^{(L, n)}(t)=\sum_{i=1}^{p}\left(b_{l i j}^{(L, n)}-b_{i j}^{L}(t)\right) \cdot w_{l i}^{(* I, n)} \text { and } h_{l j}^{(U, n)}(t)=\sum_{i=1}^{p}\left(b_{l i j}^{(U, n)}-b_{i j}^{U}(t)\right) \cdot w_{l i}^{(* I I, n)} .
$$

For $l=1, \cdots, n$, we define

$$
\begin{aligned}
& \pi_{l}^{(L L, n)}=\left\{\begin{array}{ll}
\max _{j \in P} \sup _{t \in F_{l}^{(n)}}\left[h_{l j}^{(L, n)}(t)+a_{j}^{L}(t)-a_{l j}^{(L, n)}\right], & \text { if } P \neq \varnothing \\
0, & \text { if } P=\varnothing
\end{array}\right\} \\
& \pi_{l}^{(L U, n)}=\left\{\begin{array}{ll}
\max _{j \in N} \sup _{t \in F_{l}^{(n)}}\left[h_{l j}^{(L, n)}(t)+a_{j}^{U}(t)-a_{l j}^{(U, n)}\right], & \text { if } N \neq \varnothing \\
0, & \text { if } N=\varnothing
\end{array}\right\} \\
& \pi_{l}^{(U L, n)}=\left\{\begin{array}{ll}
\max _{j \in N} \sup _{t \in F_{l}^{(n)}}\left[h_{l j}^{(U, n)}(t)+a_{j}^{L}(t)-a_{l j}^{(L, n)}\right], & \text { if } N \neq \varnothing \\
0, & \text { if } N=\varnothing
\end{array}\right\}
\end{aligned}
$$




$$
\pi_{l}^{(U U, n)}=\left\{\begin{array}{ll}
\max _{j \in P} \sup _{t \in F_{l}^{(n)}}\left[h_{l j}^{(U, n)}(t)+a_{j}^{U}(t)-a_{l j}^{(U, n)}\right] & \text { if } P \neq \varnothing \\
0, & \text { if } P=\varnothing
\end{array}\right\} .
$$

We also define

$$
\pi_{l}^{(L, n)}=\max \left\{\pi_{l}^{(L L, n)}, \pi_{l}^{(L U, n)}\right\} \text { and } \pi_{l}^{(U, n)}=\max \left\{\pi_{l}^{(U L, n)}, \pi_{l}^{(U U, n)}\right\} .
$$

From Lemma 2, it follows that

$$
\lim _{n \rightarrow \infty} \pi_{l}^{(L, n)}=0=\lim _{n \rightarrow \infty} \pi_{l}^{(L, n)} .
$$

For $l=1, \cdots, n$, we define

$$
\mathfrak{b}_{l}^{(L, n)}=\min _{j=1, \cdots, q}\left\{\sum_{i=1}^{p} \inf _{t \in \bar{E}_{l}^{(n)}} b_{i j}^{L}(t)\right\} \text { and } \mathfrak{b}_{l}^{(U, n)}=\min _{j=1, \cdots, q}\left\{\sum_{i=1}^{p} \inf _{t \in \bar{E}_{l}^{(n)}} b_{i j}^{U}(t)\right\} .
$$

Since $b_{i j}^{L}$ is increasing on $[0,1]$ and $b_{i j}^{U}$ is decreasing on $[0,1]$, it follows that, for $l=1, \cdots, n$,

$$
\mathfrak{b}_{l}^{(L, n)}=\min _{j=1, \cdots, q}\left\{\sum_{i=1}^{p} b_{i j}^{L}\left(\frac{l-1}{n}\right)\right\} \text { and } \mathfrak{b}_{l}^{(U, n)}=\min _{j=1, \cdots, q}\left\{\sum_{i=1}^{p} b_{i j}^{U}\left(\frac{l}{n}\right)\right\} .
$$

According to (15) and (16), we define

$$
\sigma^{L}=\min _{j=1, \cdots, q}\left\{\sum_{i=1}^{p} \inf _{t \in[0,1]} b_{i j}^{L}(t)\right\}>0 \text { and } \sigma^{U}=\min _{j=1, \cdots, q}\left\{\sum_{i=1}^{p} \inf _{t \in[0,1]} b_{i j}^{U}(t)\right\}>0 .
$$

It is clear to see that

$$
\mathfrak{b}_{l}^{(L, n)} \geq \sigma^{L}>0 \text { and } \mathfrak{b}_{l}^{(U, n)} \geq \sigma^{U}>0 \text { for } l=1, \cdots, n .
$$

We also define the real-valued functions $\mathfrak{v}^{(L, n)}$ and $\mathfrak{v}^{(U, n)}$ on $[0,1]$ by

$$
\mathfrak{v}^{(L, n)}(t)= \begin{cases}\mathfrak{b}_{l}^{(L, n)}, & \text { if } t \in F_{l}^{(n)} \text { for } l=1, \cdots, n \\ \mathfrak{b}_{n}^{(L, n)}, & \text { if } t=1\end{cases}
$$

and

$$
\mathfrak{v}^{(U, n)}(t)= \begin{cases}\mathfrak{b}_{l}^{(U, n)}, & \text { if } t \in F_{l}^{(n)} \text { for } l=1, \cdots, n \\ \mathfrak{b}_{n}^{(U, n)}, & \text { if } t=1 .\end{cases}
$$

Now we define the real numbers

$$
\kappa^{(L, n)}=\max \left\{\max _{j \in P}\left\{h_{n j}^{(L, n)}(1)+a_{j}^{L}(1)-a_{n j}^{(L, n)}\right\}, \max _{j \in N}\left\{h_{n j}^{(L, n)}(1)+a_{j}^{U}(1)-a_{n j}^{(U, n)}\right\}\right\}
$$

and

$$
\kappa^{(U, n)}=\max \left\{\max _{j \in N}\left\{h_{n j}^{(U, n)}(1)+a_{j}^{L}(1)-a_{n j}^{(L, n)}\right\}, \max _{j \in P}\left\{h_{n j}^{(U, n)}(1)+a_{j}^{U}(1)-a_{n j}^{(U, n)}\right\}\right\},
$$

where we assume

$$
\max _{j \in P}\left\{h_{n j}^{(L, n)}(1)+a_{j}^{L}(1)-a_{n j}^{(L, n)}\right\}=0=\max _{j \in P}\left\{h_{n j}^{(U, n)}(1)+a_{j}^{U}(1)-a_{n j}^{(U, n)}\right\} \text { for } P=\varnothing
$$


and

$$
\max _{j \in N}\left\{h_{n j}^{(L, n)}(1)+a_{j}^{U}(1)-a_{n j}^{(U, n)}\right\}=0=\max _{j \in N}\left\{h_{n j}^{(U, n)}(1)+a_{j}^{L}(1)-a_{n j}^{(L, n)}\right\} \text { for } N=\varnothing .
$$

Then we define the real-valued functions $\mathfrak{p}^{(L, n)}$ and $\mathfrak{p}^{(U, n)}$ on $[0,1]$ by

$$
\mathfrak{p}^{(L, n)}(t)= \begin{cases}\pi_{l}^{(L, n)}, & \text { if } t \in F_{l}^{(n)} \text { for } l=1, \cdots, n \\ \kappa^{(L, n),} & \text { if } t=1\end{cases}
$$

and

$$
\mathfrak{p}^{(U, n)}(t)= \begin{cases}\pi_{l}^{(U, n),} & \text { if } t \in F_{l}^{(n)} \text { for } l=1, \cdots, n \\ \kappa^{(U, n),} & \text { if } t=1 .\end{cases}
$$

We also define the real-valued functions $\mathfrak{f}^{(L, n)}$ and $\mathfrak{f}^{(U, n)}$ on $[0,1]$ by

$$
\mathfrak{f}^{(L, n)}(t)=\frac{\mathfrak{p}^{(L, n)}(t)}{\mathfrak{v}^{(L, n)}(t)} \text { and } \mathfrak{f}^{(U, n)}(t)=\frac{\mathfrak{p}^{(U, n)}(t)}{\mathfrak{v}^{(U, n)}(t)}
$$

According to Lemma 3, we can similarly obtain the following lemma.

Lemma 10. For $l=1, \cdots, n, j=1, \cdots, q$ and $t \in F_{l}^{(n)}$, we have

$$
\mathfrak{f}^{(L, n)}(t) \cdot \sum_{i=1}^{p} b_{i j}^{L}(t) \geq\left\{\begin{array}{l}
h_{l j}^{(L, n)}(t)+a_{j}^{L}(t)-a_{l j}^{(L, n)} \text { for } j \in P \\
h_{l j}^{(L, n)}(t)+a_{j}^{U}(t)-a_{l j}^{(U, n)} \text { for } j \in N
\end{array}\right\}
$$

and

$$
\mathfrak{f}^{(U, n)}(t) \cdot \sum_{i=1}^{p} b_{i j}^{U}(t) \geq\left\{\begin{array}{l}
h_{l j}^{(U, n)}(t)+a_{j}^{L}(t)-a_{l j}^{(L, n)} \text { for } j \in N \\
h_{l j}^{(U, n)}(t)+a_{j}^{U}(t)-a_{l j}^{(U, n)} \text { for } j \in P
\end{array}\right\} .
$$

For $t=1$ and $j=1, \cdots, q$, we also have

$$
\mathfrak{f}^{(L, n)}(1) \cdot \sum_{i=1}^{p} b_{i j}^{L}(1) \geq\left\{\begin{array}{l}
h_{n j}^{(L, n)}(1)+a_{j}^{L}(1)-a_{n j}^{(L, n)} \text { for } j \in P \\
h_{n j}^{(L, n)}(1)+a_{j}^{U}(1)-a_{n j}^{(U, n)} \text { for } j \in N
\end{array}\right\}
$$

and

$$
\mathfrak{f}^{(U, n)}(1) \cdot \sum_{i=1}^{p} b_{i j}^{U}(1) \geq\left\{\begin{array}{l}
h_{n j}^{(U, n)}(1)+a_{j}^{L}(1)-a_{n j}^{(L, n)} \text { for } j \in N \\
h_{n j}^{(U, n)}(1)+a_{j}^{U}(1)-a_{n j}^{(U, n)} \text { for } j \in P
\end{array}\right\} .
$$

The sequences $\left\{\mathfrak{f}^{(L, n)}\right\}_{n=1}^{\infty}$ and $\left\{\mathfrak{f}^{(U, n)}\right\}_{n=1}^{\infty}$ of real-valued functions are uniformly bounded.

Instead of defining the real-valued functions $y_{i}^{(I, n)}, y_{i}^{(I I, n)}, y_{j}^{(I I I, n)}, y_{j}^{(I V, n)}$ and $y_{j}^{(V, n)}$ in (88)-(92), we define them as follows:

$$
\begin{gathered}
y_{i}^{(I, n)}(t)=\left\{\begin{array}{ll}
w_{l i}^{(* I, n)}+\mathfrak{f}^{(L, n)}(t), & \text { if } t \in F_{l}^{(n)} \text { for } l=1, \cdots, n \\
w_{n i}^{(* I, n)}+\mathfrak{f}^{(L, n)}(1), & \text { if } t=1
\end{array}\right\} \text { for } i=1, \cdots, p ; \\
y_{i}^{(I I, n)}(t)=\left\{\begin{array}{ll}
w_{l i}^{(* I I, n)}+\mathfrak{f}^{(U, n)}(t), & \text { if } t \in F_{l}^{(n)} \text { for } l=1, \cdots, n \\
w_{n i}^{(* I I, n)}+\mathfrak{f}^{(U, n)}(1), & \text { if } t=1
\end{array}\right\} \text { for } i=1, \cdots, p ; \\
y_{j}^{(I I I, n)}(t)=\left\{\begin{array}{ll}
w_{l j}^{(* I I I, n)}, & \text { if } t \in F_{l}^{(n)} \text { for } l=1, \cdots, n \\
w_{n j}^{(* I I I, n)}, & \text { if } t=1
\end{array}\right\} \text { for } j=1, \cdots, q ;
\end{gathered}
$$




$$
\begin{aligned}
& y_{j}^{(I V, n)}(t)=\left\{\begin{array}{ll}
w_{l j}^{(* I V, n),}, & \text { if } t \in F_{l}^{(n)} \text { for } l=1, \cdots, n-1 \\
w_{n-1, j}^{(* I V, n)}, & \text { if } t \in F_{n}^{(n)} \cup\{1\}=\bar{E}_{n}^{(n)}
\end{array}\right\} \text { for } j=1, \cdots, q ; \\
& y_{j}^{(V, n)}(t)=\left\{\begin{array}{ll}
w_{l j}^{(* V, n),} & \text { if } t \in F_{l}^{(n)} \text { for } l=1, \cdots, n-1 \\
w_{n-1, j}^{(* V, n)}, & \text { if } t \in F_{n}^{(n)} \cup\{1\}=\bar{E}_{n}^{(n)}
\end{array}\right\} \text { for } j=1, \cdots, q .
\end{aligned}
$$

The feasibility is presented below.

Proposition 9. The vector-valued function

$$
\mathbf{y}^{(n)}(t)=\left(\mathbf{y}^{(I, n)}(t), \mathbf{y}^{(I I, n)}(t), \mathbf{y}^{(I I I, n)}(t), \mathbf{y}^{(I V, n)}(t), \mathbf{y}^{(V, n)}(t)\right)
$$

with component functions defined in (191)-(195) is a feasible solution of problem (DNSOP $\left.{ }_{n}\right)$.

Proof. We are going to show that $\mathbf{y}^{(n)}$ satisfies all the constraints of problem (DNSOP ${ }_{n}$ ). For $j=$ $1, \cdots, q$, we define the real-valued functions $g_{l j}^{(U, n)}$ on $F_{l}^{(n)}$ for $l=1, \cdots, n-1$ and $g_{n j}^{(U, n)}$ on $\bar{E}_{n}^{(n)}=$ $F_{n}^{(n)} \cup\{1\}$ by

$$
g_{l j}^{(U, n)}(t)=\sum_{i=1}^{p}\left(b_{i j}^{U}(t)-b_{l i j}^{(U, n)}\right) \cdot w_{l i}^{(* I I, n)}+\mathfrak{f}^{(U, n)}(t) \cdot \sum_{i=1}^{p} b_{i j}^{U}(t) .
$$

Using Lemma 10, we have

$$
g_{l j}^{(U, n)}(t)=-h_{l j}^{(U, n)}(t)+\mathfrak{f}^{(U, n)}(t) \cdot \sum_{i=1}^{p} b_{i j}^{U}(t) \geq\left\{\begin{array}{l}
a_{j}^{L}(t)-a_{l j}^{(L, n)} \text { for } j \in N \\
a_{j}^{U}(t)-a_{l j}^{(U, n)} \text { for } j \in P
\end{array}\right\} .
$$

Since $1 / n$ is the length of each interval $F_{l}^{(n)}$, for $t \in F_{l}^{(n)}$ and $l=2, \cdots, n$, we see that $t-\frac{1}{n} \in F_{l-1}^{(n)}$. We consider the following cases.

- $\quad$ For $l=2, \cdots, n-1, j=1, \cdots, q$ and $t \in F_{l}^{(n)}$, i.e., $t-\frac{1}{n} \in F_{l-1}^{(n)}$, we obtain

$$
\begin{aligned}
& \sum_{i=1}^{p} b_{i j}^{U}(t) \cdot y_{i}^{(I I, n)}(t)-y_{j}^{(I I I, n)}(t)-y_{j}^{(V, n)}(t)+y_{j}^{(V, n)}\left(t-\frac{1}{n}\right) \\
& =\sum_{i=1}^{p} b_{i j}^{U}(t) \cdot w_{l i}^{(* I I, n)}+\mathfrak{f}^{(U, n)}(t) \cdot \sum_{i=1}^{p} b_{i j}^{U}(t)-w_{l j}^{(* I I I, n)}-w_{l j}^{(* V, n)}+w_{l-1, j}^{(* V, n)} \\
& \text { (by (192), (193) and (195)) } \\
& =\sum_{i=1}^{p} b_{l i j}^{(U, n)} \cdot w_{l i}^{(* I I, n)}-w_{l j}^{(* I I I, n)}-w_{l j}^{(* V, n)}+w_{l-1, j}^{(* V, n)}+g_{l j}^{(U, n)}(t)(\text { by (196)) } \\
& \geq\left\{\begin{array}{ll}
a_{l j}^{(L, n)}+g_{l j}^{(U, n)}(t) \text { for } j \in N & \text { (using the feasibility (183) for problem } \left.\left(\mathrm{ND}_{n}\right)\right) \\
a_{l j}^{(U, n)}+g_{l j}^{(U, n)}(t) \text { for } j \in P & \text { (using the feasibility (184) for problem } \left.\left(\mathrm{ND}_{n}\right)\right)
\end{array}\right\} \\
& \geq\left\{\begin{array}{l}
a_{j}^{L}(t) \text { for } j \in N \\
a_{j}^{U}(t) \text { for } j \in P
\end{array}\right\} \quad(\text { by (197)). }
\end{aligned}
$$


- $\quad$ For $l=1, j=1, \cdots, q$ and $t \in F_{1}^{(n)}$, we obtain

$$
\begin{aligned}
& \sum_{i=1}^{p} b_{i j}^{U}(t) \cdot y_{i}^{(I I, n)}(t)-y_{j}^{(I I I, n)}(t)-y_{j}^{(V, n)}(t) \\
& \quad=\sum_{i=1}^{p} b_{i j}^{U}(t) \cdot w_{1 i}^{(* I I, n)}+\sum_{i=1}^{p} b_{i j}^{U}(t) \cdot f^{(U, n)}(t)-w_{1 j}^{(* I I I, n)}-w_{1 j}^{(* V, n)} \text { (by (192), (193) and (195)) } \\
& \quad=\sum_{i=1}^{p} b_{1 i j}^{(U, n)} \cdot w_{1 i}^{(* I I, n)}-w_{1 j}^{(* I I I, n)}-w_{1 j}^{(* V, n)}+g_{1 j}^{(U, n)}(t)(\text { by (196)) } \\
& \quad \geq\left\{\begin{array}{l}
a_{1 j}^{(L, n)}+g_{1 j}^{(U, n)}(t) \text { for } j \in N\left(\text { using the feasibility (179) for problem }\left(\mathrm{ND}_{n}\right)\right) \\
a_{1 j}^{(U, n)}+g_{1 j}^{(U, n)}(t) \text { for } j \in P\left(\text { using the feasibility (180) for problem }\left(\mathrm{ND}_{n}\right)\right)
\end{array}\right\} \\
& \quad \geq\left\{\begin{array}{l}
a_{j}^{L}(t) \text { for } j \in N \\
a_{j}^{U}(t) \text { for } j \in P
\end{array}\right\} \text { (by (197)). }
\end{aligned}
$$

- $\quad$ For $l=n, j=1, \cdots, q$ and $t \in F_{n}^{(n)}$, i.e., $t-\frac{1}{n} \in F_{n-1}^{(n)}$, we obtain

$$
\begin{aligned}
& \sum_{i=1}^{p} b_{i j}^{U}(t) \cdot y_{i}^{(I I, n)}(t)-y_{j}^{(I I I, n)}(t)+y_{j}^{(V, n)}\left(t-\frac{1}{n}\right) \\
& =\sum_{i=1}^{p} b_{i j}^{U}(t) \cdot w_{n i}^{(* I I, n)}+\sum_{i=1}^{p} b_{i j}^{U}(t) \cdot \mathfrak{f}^{(U, n)}(t)-w_{n j}^{(* I I I, n)}+w_{n-1, j}^{(* V, n)} \text { (by (192), (193) and (195)) } \\
& =\sum_{i=1}^{p} b_{n i j}^{(U, n)} \cdot w_{n i}^{(* I I, n)}-w_{n j}^{(* I I I, n)}+w_{n-1, j}^{(* V, n)}+g_{n j}^{(U, n)}(t)(\text { by }(196)) \\
& \geq\left\{\begin{array}{ll}
a_{n j}^{(L, n)}+g_{n j}^{(U, n)}(t) \text { for } j \in N & \text { (using the feasibility (187) for problem } \left.\left(\mathrm{ND}_{n}\right)\right) \\
a_{n j}^{(U, n)}+g_{n j}^{(U, n)}(t) \text { for } j \in P & \text { (using the feasibility (188) for problem } \left.\left(\mathrm{ND}_{n}\right)\right)
\end{array}\right\} \\
& \geq\left\{\begin{array}{l}
a_{j}^{L}(t) \text { for } j \in N \\
a_{j}^{U}(t) \text { for } j \in P
\end{array}\right\} \quad(\text { by (197)). }
\end{aligned}
$$

- $\quad$ For $l=n, j=1, \cdots, q$ and $t=1$, it is clear to see that $1-\frac{1}{n} \in F_{n}^{(n)}$. We obtain

$$
\begin{aligned}
& \sum_{i=1}^{p} b_{i j}^{U}(1) \cdot y_{i}^{(I I, n)}(1)-y_{j}^{(I I I, n)}(1)+y_{j}^{(V, n)}\left(1-\frac{1}{n}\right) \\
& \quad=\sum_{i=1}^{p} b_{i j}^{U}(1) \cdot w_{n i}^{(* I I, n)}+\sum_{i=1}^{p} b_{i j}^{U}(1) \cdot \mathfrak{f}^{(U, n)}(1)-w_{n j}^{(* I I I, n)}+w_{n-1, j}^{(* V, n)} \text { (by (192), (193) and (195)) }
\end{aligned}
$$




$$
\begin{aligned}
& =\sum_{i=1}^{p} b_{n i j}^{(U, n)} \cdot w_{n i}^{(* I I, n)}-w_{n j}^{(* I I I, n)}+w_{n-1, j}^{(* V, n)}+g_{n j}^{(U, n)}(1)(\text { by (196)) } \\
& \geq\left\{\begin{array}{ll}
a_{n j}^{(L, n)}+g_{n j}^{(U, n)}(1) \text { for } j \in N & \text { (using the feasibility (187) for problem } \left.\left(\mathrm{ND}_{n}\right)\right) \\
a_{n j}^{(U, n)}+g_{n j}^{(U, n)}(1) \text { for } j \in P & \text { (using the feasibility (188) for problem } \left.\left(\mathrm{ND}_{n}\right)\right)
\end{array}\right\} \\
& \geq\left\{\begin{array}{l}
a_{j}^{L}(1) \text { for } j \in N \\
a_{j}^{U}(1) \text { for } j \in P
\end{array}\right\} \quad(\text { by (197)). }
\end{aligned}
$$

On the other hand, for $l=1, \cdots, n-1$ and $j=1, \cdots, q$, we define the real-valued functions $g_{l j}^{(L, n)}$ on $F_{l}^{(n)}$ and $g_{n j}^{(L, n)}$ on $\bar{E}_{n}^{(n)}$ by

$$
g_{l j}^{(L, n)}(t)=\sum_{i=1}^{p}\left(b_{i j}^{L}(t)-b_{l i j}^{(L, n)}\right) \cdot w_{l i}^{(* I, n)}+\mathfrak{f}^{(L, n)}(t) \cdot \sum_{i=1}^{p} b_{i j}^{L}(t) .
$$

Using Lemma 10, we have

$$
g_{l j}^{(L, n)}(t)=-h_{l j}^{(L, n)}(t)+\mathfrak{f}^{(L, n)}(t) \cdot \sum_{i=1}^{p} b_{i j}^{L}(t) \geq\left\{\begin{array}{l}
a_{j}^{L}(t)-a_{l j}^{(L, n)} \text { for } j \in P \\
a_{j}^{U}(t)-a_{l j}^{(U, n)} \text { for } j \in N
\end{array}\right\} .
$$

We consider the following cases.

- $\quad$ For $l=2, \cdots, n-1, j=1, \cdots, q$ and $t \in F_{l}^{(n)}$, i.e., $t-\frac{1}{n} \in F_{l-1}^{(n)}$, we obtain

$$
\begin{aligned}
& \sum_{i=1}^{p} b_{i j}^{L}(t) y_{i}^{(I, n)}(t)+y_{j}^{(I I I, n)}(t)+y_{j}^{(I V, n)}(t)-y_{j}^{(I V, n)}\left(t-\frac{1}{n}\right) \\
&= \sum_{i=1}^{p} b_{i j}^{L}(t) \cdot w_{l i}^{(* I, n)}+\mathfrak{f}^{(L, n)}(t) \cdot \sum_{i=1}^{p} b_{i j}^{L}(t)+w_{l j}^{(* I I I, n)}+w_{l j}^{(* I V, n)}-w_{l-1, j}^{(* I V, n)} \\
&(\text { by }(191),(193) \text { and }(194))
\end{aligned}
$$

- $\quad$ For $l=1, j=1, \cdots, q$ and $t \in F_{1}^{(n)}$, we obtain

$$
\sum_{i=1}^{p} b_{i j}^{L}(t) \cdot y_{i}^{(I, n)}(t)+y_{j}^{(I I I, n)}(t)+y_{j}^{(I V, n)}(t)
$$




$$
\begin{aligned}
& =\sum_{i=1}^{p} b_{i j}^{L}(t) \cdot w_{1 i}^{(* I, n)}+\sum_{i=1}^{p} b_{i j}^{L}(t) \cdot \mathfrak{f}^{(L, n)}(t)+w_{1 j}^{(* I I I, n)}+w_{1 j}^{(* I V, n)}(\text { by (191), (193) and (194)) } \\
& =\sum_{i=1}^{p} b_{1 i j}^{(L, n)} \cdot w_{1 i}^{(* I, n)}+w_{1 j}^{(* I I I, n)}+w_{1 j}^{(* I V, n)}+g_{1 j}^{(L, n)}(t)(\text { by (198)) } \\
& \geq\left\{\begin{array}{l}
\left.a_{1 j}^{(L, n)}+g_{1 j}^{(L, n)}(t) \text { for } j \in P \text { (using the feasibility (177) for problem }\left(\mathrm{ND}_{n}\right)\right) \\
\left.a_{1 j}^{(U, n)}+g_{1 j}^{(L, n)}(t) \text { for } j \in N \text { (using the feasibility (178) for problem }\left(\mathrm{ND}_{n}\right)\right)
\end{array}\right\} \\
& \geq\left\{\begin{array}{l}
a_{j}^{L}(t) \text { for } j \in P \\
a_{j}^{U}(t) \text { for } j \in N
\end{array}\right\} \text { (by (199)). }
\end{aligned}
$$

- $\quad$ For $l=n, j=1, \cdots, q$ and $t \in F_{n}^{(n)}$, i.e., $t-\frac{1}{n} \in F_{n-1}^{(n)}$, we obtain

$$
\begin{aligned}
& \sum_{i=1}^{p} b_{i j}^{L}(t) \cdot y_{i}^{(I, n)}(t)+y_{j}^{(I I I, n)}(t)-y_{j}^{(I V, n)}\left(t-\frac{1}{n}\right) \\
& \quad=\sum_{i=1}^{p} b_{i j}^{L}(t) \cdot w_{n i}^{(* I, n)}+\sum_{i=1}^{p} b_{i j}^{L}(t) \cdot f^{(L, n)}(t)+w_{n j}^{(* I I, n)}-w_{n-1, j}^{(* I V, n)}(\text { by }(191),(193) \text { and }(194)) \\
& \quad=\sum_{i=1}^{p} b_{n i j}^{(L, n)} \cdot w_{n i}^{(* I, n)}+w_{n j}^{(* I I I, n)}-w_{n-1, j}^{(* I V, n)}+g_{n j}^{(L, n)}(t)(\text { by }(198)) \\
& \quad \geq\left\{\begin{array}{l}
\left.a_{n j}^{(L, n)}+g_{n j}^{(L, n)}(t) \text { for } j \in P \quad \text { (using the feasibility (185) for problem }\left(\mathrm{ND}_{n}\right)\right) \\
\left.a_{n j}^{(U, n)}+g_{n j}^{(L, n)}(t) \text { for } j \in N \quad \text { (using the feasibility (186) for problem }\left(\mathrm{ND}_{n}\right)\right)
\end{array}\right\} \\
& \quad \geq\left\{\begin{array}{l}
a_{j}^{L}(t) \text { for } j \in P \\
a_{j}^{U}(t) \text { for } j \in N
\end{array}\right\} \quad \text { (by (199)). }
\end{aligned}
$$

- $\quad$ For $l=n, j=1, \cdots, q$ and $t=1$, it is clear to see that $1-\frac{1}{n} \in F_{n}^{(n)}$. We obtain

$$
\begin{aligned}
& \sum_{i=1}^{p} b_{i j}^{L}(1) \cdot y_{i}^{(I, n)}(1)+y_{j}^{(I I I, n)}(1)-y_{j}^{(I V, n)}\left(1-\frac{1}{n}\right) \\
& \quad=\sum_{i=1}^{p} b_{i j}^{L}(1) \cdot w_{n i}^{(* I, n)}+\sum_{i=1}^{p} b_{i j}^{L}(1) \cdot \mathfrak{f}^{(L, n)}(1)+w_{n j}^{(* I I I, n)}-w_{n-1, j}^{(* I V, n)} \text { (by (191), (193) and (194)) } \\
& \quad=\sum_{i=1}^{p} b_{n i j}^{(L, n)} \cdot w_{n i}^{(* I, n)}+w_{n j}^{(* I I I, n)}-w_{n-1, j}^{(* I V, n)}+g_{n j}^{(L, n)}(1)(\mathrm{by}(198))
\end{aligned}
$$




$$
\begin{aligned}
& \geq\left\{\begin{array}{lc}
a_{n j}^{(L, n)}+g_{n j}^{(L, n)}(1) \text { for } j \in P & \text { (using the feasibility (185) for problem } \left.\left(\mathrm{ND}_{n}\right)\right) \\
a_{n j}^{(U, n)}+g_{n j}^{(L, n)}(1) \text { for } j \in N & \text { (using the feasibility (186) for problem } \left.\left(\mathrm{ND}_{n}\right)\right)
\end{array}\right\} \\
& =\left\{\begin{array}{l}
a_{j}^{L}(1) \text { for } j \in P \\
a_{j}^{U}(1) \text { for } j \in N
\end{array}\right\} \quad \text { (by (199)). }
\end{aligned}
$$

This completes the proof.

From (69), we also have

$$
V\left(\mathrm{DNSOP}_{n}\right) \geq V\left(\mathrm{NSOP}_{n}\right) \geq V(\mathrm{NSOP}) \geq V\left(\mathrm{NP}_{n}\right)=V\left(\mathrm{ND}_{n}\right) .
$$

Theorem 9. Given an optimal solution $\mathbf{z}^{(* n)}$ of problem $\left(\mathrm{NP}_{n}\right)$ and an optimal solution $\mathbf{w}^{(* n)}$ of problem $\left(\mathrm{ND}_{n}\right)$, we define

$$
\mathbf{x}^{(n)}=\left(\mathbf{x}^{(L, n)}, \mathbf{x}^{(U, n)}\right) \text { and } \mathbf{y}^{(n)}(t)=\left(\mathbf{y}^{(I, n)}(t), \mathbf{y}^{(I I, n)}(t), \mathbf{y}^{(I I I, n)}(t), \mathbf{y}^{(I V, n)}(t), \mathbf{y}^{(V, n)}(t)\right)
$$

according to (62) and (63) and (191)-(195), respectively. Then the following statements hold true.

(i) We have

$$
\limsup _{n \rightarrow \infty} V\left(\mathrm{DNSOP}_{n}\right)=\limsup _{n \rightarrow \infty} V\left(\mathrm{ND}_{n}\right)
$$

and

$$
0 \leq V\left(\mathrm{DNSOP}_{n}\right)-V\left(\mathrm{ND}_{n}\right) \leq \varepsilon_{n}^{\circ}
$$

where

$$
\begin{aligned}
\varepsilon_{n}^{\circ}= & -V\left(\mathrm{ND}_{n}\right)+\sum_{l=1}^{n} \sum_{i=1}^{p}\left[\left(w_{l i}^{(* I, n)}+\frac{\pi_{l}^{(L, n)}}{\mathfrak{b}_{l}^{(L, n)}}\right) \cdot \int_{\bar{E}_{l}^{(n)}} c_{i}^{L}(t) d t\right] \\
& +\sum_{l=1}^{n} \sum_{i=1}^{p}\left[\left(w_{l i}^{(* I I, n)}+\frac{\pi_{l}^{(U, n)}}{\mathfrak{b}_{l}^{(U, n)}}\right) \cdot \int_{\bar{E}_{l}^{(n)}} c_{i}^{U}(t) d t\right]
\end{aligned}
$$

satisfying $\varepsilon_{n}^{\circ} \rightarrow 0$ as $n \rightarrow \infty$. Moreover, there exist two convergent subsequences $\left\{V\left(\mathrm{DNSOP}_{n_{k}}\right)\right\}_{k=1}^{\infty}$ and $\left\{V\left(\mathrm{ND}_{n_{k}}\right)\right\}_{k=1}^{\infty}$ of $\left\{V\left(\mathrm{DNSOP}_{n}\right)\right\}_{n=1}^{\infty}$ and $\left\{V\left(\mathrm{ND}_{n}\right)\right\}_{n=1}^{\infty}$, respectively, such that

$$
\lim _{k \rightarrow \infty} V\left(\mathrm{DNSOP}_{n_{k}}\right)=\lim _{k \rightarrow \infty} V\left(\mathrm{ND}_{n_{k}}\right) .
$$

(ii) (Asymptotic No Duality Gap). We have

$$
V(\mathrm{NSOP})=\limsup _{n \rightarrow \infty} V\left(\mathrm{DNSOP}_{n}\right)=\limsup _{n \rightarrow \infty} V\left(\mathrm{NSOP}_{n}\right)=\limsup _{n \rightarrow \infty} V\left(\mathrm{ND}_{n}\right)=\limsup _{n \rightarrow \infty} V\left(\mathrm{NP}_{n}\right)
$$

and

$$
V(\mathrm{NSOP})=\lim _{k \rightarrow \infty} V\left(\mathrm{DNSOP}_{n_{k}}\right)=\lim _{k \rightarrow \infty} V\left(\mathrm{NSOP}_{n_{k}}\right)=\lim _{k \rightarrow \infty} V\left(\mathrm{ND}_{n_{k}}\right)=\lim _{k \rightarrow \infty} V\left(\mathrm{NP}_{n_{k}}\right)
$$

and

$$
0 \leq V(\mathrm{NSOP})-V\left(\mathrm{NP}_{n}\right) \leq \varepsilon_{n}^{\circ}
$$


Proof. To prove part (i), we have

$$
\begin{aligned}
0 \leq & V\left(\operatorname{DNSOP}_{n}\right)-V\left(\mathrm{ND}_{n}\right)(\text { by }(200)) \\
= & V\left(\operatorname{DNSOP}_{n}\right)-\sum_{i=1}^{p} \sum_{l=1}^{n} \frac{1}{n} \cdot c_{l i}^{(L, n)} \cdot w_{l i}^{(* I, n)}-\sum_{i=1}^{p} \sum_{l=1}^{n} \frac{1}{n} \cdot c_{l i}^{(U, n)} \cdot w_{l i}^{(* I, n)} \\
= & V\left(\operatorname{DNSOP}_{n}\right)-\sum_{l=1}^{n} \sum_{i=1}^{p} \int_{\bar{E}_{l}^{(n)}} c_{l i}^{(L, n)} \cdot w_{l i}^{(* I, n)} d t-\sum_{l=1}^{n} \sum_{i=1}^{p} \int_{\bar{E}_{l}^{(n)}} c_{l i}^{(U, n)} \cdot w_{l i}^{(* I I, n)} \\
\leq & \sum_{i=1}^{p} \int_{0}^{1} c_{i}^{L}(t) \cdot y_{i}^{(I, n)}(t) d t+\sum_{i=1}^{p} \int_{0}^{1} c_{i}^{U}(t) \cdot y_{i}^{(I I, n)}(t) d t \\
& -\sum_{l=1}^{n} \sum_{i=1}^{p} \int_{\bar{E}_{l}^{(n)}} c_{l i}^{(L, n)} \cdot w_{l i}^{(* I, n)} d t-\sum_{l=1}^{n} \sum_{i=1}^{p} \int_{\bar{E}_{l}^{(n)}} c_{l i}^{(U, n)} \cdot w_{l i}^{(* I I, n)} \text { (by Proposition 9) } \\
= & \sum_{i=1}^{p} \sum_{l=1}^{n} \int_{\bar{E}_{l}^{(n)}}\left(c_{i}^{L}(t)-c_{l i}^{(L, n)}\right) \cdot w_{l i}^{(* I, n)} d t+\sum_{i=1}^{p} \int_{0}^{1} \mathfrak{f}^{(L, n)}(t) \cdot c_{i}^{L}(t) d t \\
& +\sum_{i=1}^{p} \sum_{l=1}^{n} \int_{\bar{E}_{l}^{(n)}}\left(c_{i}^{U}(t)-c_{l i}^{(U, n)}\right) \cdot w_{l i}^{(* I I, n)} d t+\sum_{i=1}^{p} \int_{0}^{1} \mathfrak{f}^{(U, n)}(t) \cdot c_{i}^{U}(t) d t \text { (using (191) and (192)) } \\
\equiv & \varepsilon_{n}^{\circ}
\end{aligned}
$$

which implies

$$
V\left(\mathrm{ND}_{n}\right) \leq V\left(\mathrm{DNSOP}_{n}\right) \leq V\left(\mathrm{ND}_{n}\right)+\varepsilon_{n}^{\circ}
$$

Now we have

$$
\begin{aligned}
0 & \leq \int_{0}^{1} \mathfrak{f}^{(L, n)}(t) \cdot c_{i}^{L}(t) d t=\sum_{l=1}^{n} \int_{\bar{E}_{l}^{(n)}} \frac{\pi_{l}^{(L, n)}}{\mathfrak{b}_{l}^{(L, n)}} \cdot c_{i}^{L}(t) d t \leq \sum_{l=1}^{n} \frac{1}{n} \cdot \frac{\pi_{l}^{(L, n)}}{\sigma^{L}} \cdot \mu^{L}(\operatorname{using}(190) \text { and (37)) } \\
& =\frac{\pi_{l}^{(L, n)}}{\sigma^{L}} \cdot \mu^{L} \rightarrow 0 \text { as } n \rightarrow \infty(\operatorname{using}(189))
\end{aligned}
$$

and

$$
\begin{aligned}
0 & \leq \int_{0}^{1} \mathfrak{f}^{(U, n)}(t) \cdot c_{i}^{U}(t) d t=\sum_{l=1}^{n} \int_{\bar{E}_{l}^{(n)}} \frac{\pi_{l}^{(U, n)}}{\mathfrak{b}_{l}^{(U, n)}} \cdot c_{i}^{U}(t) d t \leq \sum_{l=1}^{n} \frac{1}{n} \cdot \frac{\pi_{l}^{(U, n)}}{\sigma^{U}} \cdot \mu^{U} \\
& =\frac{\pi_{l}^{(U, n)}}{\sigma^{U}} \cdot \mu^{U} \rightarrow 0 \text { as } n \rightarrow \infty(\operatorname{using}(189))
\end{aligned}
$$

Therefore we conclude that $\varepsilon_{n}^{\circ} \rightarrow 0$ as $n \rightarrow \infty$. Moreover the error $\varepsilon_{n}^{\circ}$ can be rewritten as

$$
\begin{aligned}
\varepsilon_{n}^{\circ}= & -V\left(\mathrm{ND}_{n}\right)+\sum_{l=1}^{n} \sum_{i=1}^{p} \int_{\bar{E}_{l}^{(n)}} c_{i}^{L}(t) \cdot w_{l i}^{(* I, n)} d t+\sum_{l=1}^{n} \sum_{i=1}^{p} \int_{\bar{E}_{l}^{(n)}} c_{i}^{U}(t) \cdot w_{l i}^{(* I I, n)} d t \\
& +\sum_{l=1}^{n} \sum_{i=1}^{p} \int_{\bar{E}_{l}^{(n)}} \frac{\pi_{l}^{(L, n)}}{\mathfrak{b}_{l}^{(L, n)}} \cdot c_{i}^{L}(t) d t+\sum_{l=1}^{n} \sum_{i=1}^{p} \int_{\bar{E}_{l}^{(n)}} \frac{\pi_{l}^{(U, n)}}{\mathfrak{b}_{l}^{(U, n)}} \cdot c_{i}^{U}(t) d t
\end{aligned}
$$

which implies the expression (202). The remaining proof can follow the similar argument in the proof of Theorem 3. This completes the proof. 
For the nonnegative problem NSOP, we can also use (106) to obtain the following error estimation

$$
\begin{aligned}
\varepsilon_{n}= & -V\left(\mathrm{D}_{n}\right)+\sum_{l=1}^{n} \sum_{i=1}^{p}\left[\left(w_{l i}^{(* I, n)}+\max \left\{\frac{\pi_{l}^{(L, n)}}{\mathfrak{b}_{l}^{(L, n)}}, \frac{\pi_{l}^{(U, n)}}{\mathfrak{b}_{l}^{(U, n)}}\right\}\right) \cdot \int_{\bar{E}_{l}^{(n)}} c_{i}^{L}(t) d t\right] \\
& +\sum_{l=1}^{n} \sum_{i=1}^{p}\left[\left(w_{l i}^{(* I I, n)}+\max \left\{\frac{\pi_{l}^{(L, n)}}{\mathfrak{b}_{l}^{(L, n)}}, \frac{\pi_{l}^{(U, n)}}{\mathfrak{b}_{l}^{(U, n)}}\right\}\right) \cdot \int_{\bar{E}_{l}^{(n)}} c_{i}^{U}(t) d t\right],
\end{aligned}
$$

where $\pi_{l}^{(L, n)}=\pi_{l}^{(P N, n)}, \pi_{l}^{(U, n)}=\pi_{l}^{(N P, n)}, \mathfrak{b}_{l}^{(L, n)}=\mathfrak{b}_{l}^{(P N, n)}$ and $\mathfrak{b}_{l}^{(U, n)}=\mathfrak{b}_{l}^{(N P, n)}$ by assuming $N_{i}=\varnothing$ for $i=1, \cdots, p$. From (202) and (208), it is clear to see that $\varepsilon_{n}^{\circ} \leq \varepsilon_{n}$, which means that we can have the tighter bound of error estimation for the nonnegative problem NSOP. In other words, when all of the fuzzy numbers in the constraints are assumed to be nonnegative, we shall calculate the tighter bound $\varepsilon_{n}^{\circ}$ presented in (202) instead of calculating the error bound $\varepsilon_{n}$ presented in (208).

The remaining results for the nonnegative problem NSOP, including the existence of optimal solutions can be similarly obtained. We omit the details here. We can also see that the real-valued functions $y_{i}^{(I, n)}, y_{i}^{(I I, n)}, y_{j}^{(I I I, n)}, y_{j}^{(I V, n)}$ and $y_{j}^{(V, n)}$ for $i=1, \cdots, p$ and $j=1, \cdots, q$ defined in (191)-(195) have the simple forms that are clearly not the special case of the forms presented in (88)-(92). These simple forms of real-valued functions can also save the computational resources.

\section{Computational Procedure}

In the sequel, we are going to present the computational procedure to obtain the error estimation and the approximate solutions of problem SOP. It is clear to see that the approximate solutions will be the step functions. According to Theorem 4, it is possible to obtain the appropriate step functions so that the corresponding objective function value is close enough to the supremum $V(\mathrm{SOP})$ when $n$ is taken to be sufficiently large. In other words, the computational procedure will obtain the $\epsilon$-optimal solution of problem SOP by referring to Theorem 5 .

In order to calculate the error $\varepsilon_{n}$ between the approximate objective value and the optimal objective value as shown in Theorem 3 , we need to obtain $\pi_{l}^{(P N, n)}$ and $\pi_{l}^{(N P, n)}$. By referring to (72)-(75), we need to solve

$$
\sup _{t \in F_{l}^{(n)}}\left\{h_{l j}^{(P N, n)}(t)+a_{j}^{L}(t)\right\} \text { and } \sup _{t \in F_{l}^{(n)}}\left\{h_{l j}^{(N P, n)}(t)+a_{j}^{U}(t)\right\} \text { for } j \in P .
$$

and

$$
\sup _{t \in F_{l}^{(n)}}\left\{h_{l j}^{(N P, n)}(t)+a_{j}^{L}(t)\right\} \text { and } \sup _{t \in F_{l}^{(n)}}\left\{h_{l j}^{(P N, n)}(t)+a_{j}^{U}(t)\right\} \text { for } j \in N .
$$

For $l=1, \cdots, n$ and $j=1, \cdots, q$, we define

$$
\widehat{h}_{l j}^{(P N, n)}=\sum_{\left\{i: j \in P_{i}\right\}} b_{l i j}^{(L, n)} \cdot w_{l i}^{(* I, n)}+\sum_{\left\{i: j \in N_{i}\right\}} b_{l i j}^{(U, n)} \cdot w_{l i}^{(* I I, n)}
$$

and

$$
\widehat{h}_{l j}^{(N P, n)}=\sum_{\left\{i: j \in N_{i}\right\}} b_{l i j}^{(L, n)} \cdot w_{l i}^{(* I, n)}+\sum_{\left\{i: j \in P_{i}\right\}} b_{l i j}^{(U, n)} \cdot w_{l i}^{(* I I, n)} .
$$

For $j=1, \cdots, q$ and $t \in F_{l}^{(n)}$ for $l=1, \cdots, n-1$ and $t \in F_{n}^{(n)} \cup\{1\}=\bar{E}_{n}^{(n)}$ for $l=n$, we define

$$
\breve{h}_{l j}^{(P N, n)}(t)=-\sum_{\left\{i: j \in P_{i}\right\}} b_{i j}^{L}(t) \cdot w_{l i}^{(* I, n)}-\sum_{\left\{i: j \in N_{i}\right\}} b_{i j}^{U}(t) \cdot w_{l i}^{(* I I, n)}
$$


and

$$
\breve{h}_{l j}^{(N P, n)}(t)=-\sum_{\left\{i: j \in N_{i}\right\}} b_{i j}^{L}(t) \cdot w_{l i}^{(* I, n)}-\sum_{\left\{i: j \in P_{i}\right\}} b_{i j}^{U}(t) \cdot w_{l i}^{(* I I, n)} .
$$

Then, from (70) and (71), we see that the real-valued functions $h_{l j}^{(P N, n)}$ and $h_{l j}^{(N P, n)}$ can be rewritten as

$$
h_{l j}^{(P N, n)}(t)=\widehat{h}_{l j}^{(P N, n)}+\breve{h}_{l j}^{(P N, n)}(t) \text { and } h_{l j}^{(N P, n)}(t)=\widehat{h}_{l j}^{(N P, n)}+\breve{h}_{l j}^{(N P, n)}(t) .
$$

Since the functions $a_{j}^{L}, a_{j}^{U}, b_{i j}^{L}$ and $b_{i j}^{U}$ are continuous on $\bar{E}_{l}^{(n)}$ for all $i=1, \ldots, p$ and $j=1, \cdots, q$, it follows that the functions $h_{l j}^{(P N, n)}+a_{j}^{L}$ and $h_{l j}^{(N P, n)}+a_{j}^{U}$ are also continuous on the compact interval $\bar{E}_{l}^{(n)}$. In other words, the supremum in (209) and (210) can be obtained as follows. For $j \in P$, we have

$$
\sup _{t \in F_{l}^{(n)}}\left[h_{l j}^{(P N, n)}(t)+a_{j}^{L}(t)\right]=\sup _{t \in \bar{E}_{l}^{(n)}}\left[h_{l j}^{(P N, n)}(t)+a_{j}^{L}(t)\right]=\max _{t \in \bar{E}_{l}^{(n)}}\left[h_{l j}^{(P N, n)}(t)+a_{j}^{L}(t)\right]
$$

and

$$
\sup _{t \in F_{l}^{(n)}}\left[h_{l j}^{(N P, n)}(t)+a_{j}^{U}(t)\right]=\sup _{t \in \bar{E}_{l}^{(n)}}\left[h_{l j}^{(N P, n)}(t)+a_{j}^{U}(t)\right]=\max _{t \in \bar{E}_{l}^{(n)}}\left[h_{l j}^{(N P, n)}(t)+a_{j}^{U}(t)\right] .
$$

For $j \in N$, we have

$$
\sup _{t \in F_{l}^{(n)}}\left[h_{l j}^{(P N, n)}(t)+a_{j}^{U}(t)\right]=\sup _{t \in \bar{E}_{l}^{(n)}}\left[h_{l j}^{(P N, n)}(t)+a_{j}^{U}(t)\right]=\max _{t \in \bar{E}_{l}^{(n)}}\left[h_{l j}^{(P N, n)}(t)+a_{j}^{U}(t)\right]
$$

and

$$
\sup _{t \in F_{l}^{(n)}}\left[h_{l j}^{(N P, n)}(t)+a_{j}^{L}(t)\right]=\sup _{t \in \bar{E}_{l}^{(n)}}\left[h_{l j}^{(N P, n)}(t)+a_{j}^{L}(t)\right]=\max _{t \in \bar{E}_{l}^{(n)}}\left[h_{l j}^{(N P, n)}(t)+a_{j}^{L}(t)\right] .
$$

In order to further design the computational procedure, we need to assume that the real-valued functions $a_{j}^{L}, a_{j}^{U}, b_{i j}^{L}$ and $b_{i j}^{U}$ are twice-differentiable on $[0,1]$ for the purpose of applying the Newton's method. For $j \in P$, we define the real-valued functions

$$
\bar{h}_{l j}^{(P N L, n)}(t)=h_{l j}^{(P N, n)}(t)+a_{j}^{L}(t) \text { and } \bar{h}_{l j}^{(N P U, n)}(t)=h_{l j}^{(N P, n)}(t)+a_{j}^{U}(t) .
$$

For $j \in N$, we define the real-valued functions

$$
\bar{h}_{l j}^{(N P L, n)}(t)=h_{l j}^{(N P, n)}(t)+a_{j}^{L}(t) \text { and } \bar{h}_{l j}^{(P N U, n)}(t)=h_{l j}^{(P N, n)}(t)+a_{j}^{U}(t) .
$$

From (214), we just need to solve the following simple type of optimization problem

$$
\max _{\frac{l-1}{n} \leq t \leq \frac{l}{n}} \bar{h}_{l j}^{(P N L, n)}(t)
$$

We can see that the optimal solution is

$$
t^{*}=\frac{l-1}{n} \text { or } t^{*}=\frac{l}{n} \text { or satisfying }\left.\frac{d}{d t}\left(\bar{h}_{l j}^{(P N L, n)}(t)\right)\right|_{t=t^{*}}=0 .
$$

According to (213), it follows that the optimal solution of problem (220) is

$$
t^{*}=\frac{l-1}{n} \text { or } t^{*}=\frac{l}{n} \text { or satisfying }\left.\frac{d}{d t}\left(\breve{h}_{l j}^{(P N, n)}(t)+a_{j}^{L}(t)\right)\right|_{t=t^{*}}=0 .
$$


Let $Z_{l j}^{(P N L, n)}$ denote the set of all zeros of the real-valued function $\frac{d}{d t}\left(\breve{h}_{l j}^{(P N, n)}(t)+a_{j}^{L}(t)\right)$ for $j \in P$, where $Z_{l j}^{(P N L, n)}=\left\{t_{1}^{*}, \cdots, t_{m}^{*}\right\}$ is assumed to be a finite set. Then

$$
\begin{aligned}
& \max _{t \in \bar{E}_{l}^{(n)}} \bar{h}_{l j}^{(P N L, n)}(t) \\
& \quad= \begin{cases}\max \left\{\bar{h}_{l j}^{(P N L, n)}\left(\frac{l-1}{n}\right), \bar{h}_{l j}^{(P N L, n)}\left(\frac{l}{n}\right), \max _{r=1, \cdots, m} \bar{h}_{l j}^{(P N L, n)}\left(t_{r}^{*}\right)\right\}, & \text { if } Z_{l j}^{(P N L, n)} \neq \varnothing \\
\max \left\{\bar{h}_{l j}^{(P N L, n)}\left(\frac{l-1}{n}\right), \bar{h}_{l j}^{(P N L, n)}\left(\frac{l}{n}\right)\right\}, & \text { if } Z_{l j}^{(P N L, n)}=\varnothing .\end{cases}
\end{aligned}
$$

Let $Z_{l j}^{(N P U, n)}$ denote the set of all zeros of the real-valued function $\frac{d}{d t}\left(\breve{h}_{l j}^{(N P, n)}(t)+a_{j}^{U}(t)\right)$ for $j \in P$. Then we can similarly obtain

$$
\begin{aligned}
& \max _{t \in \bar{E}_{l}^{(n)}} \bar{h}_{l j}^{(N P U, n)}(t) \\
& \quad= \begin{cases}\max \left\{\bar{h}_{l j}^{(N P U, n)}\left(\frac{l-1}{n}\right), \bar{h}_{l j}^{(N P U, n)}\left(\frac{l}{n}\right), \max _{r=1, \cdots, m} \bar{h}_{l j}^{(N P U, n)}\left(t_{r}^{*}\right)\right\}, & \text { if } Z_{l j}^{(N P U, n)} \neq \varnothing \\
\max \left\{\bar{h}_{l j}^{(N P U, n)}\left(\frac{l-1}{n}\right), \bar{h}_{l j}^{(N P U, n)}\left(\frac{l}{n}\right)\right\}, & \text { if } Z_{l j}^{(N P U, n)}=\varnothing .\end{cases}
\end{aligned}
$$

Let $Z_{l j}^{(N P L, n)}$ denote the set of all zeros of the real-valued function $\frac{d}{d t}\left(\breve{h}_{l j}^{(P N, n)}(t)+a_{j}^{L}(t)\right)$ for $j \in N$. Then we can similarly obtain

$$
\begin{aligned}
& \max _{t \in \bar{E}_{l}^{(n)}} \bar{h}_{l j}^{(N P L, n)}(t) \\
& \quad= \begin{cases}\max \left\{\bar{h}_{l j}^{(N P L, n)}\left(\frac{l-1}{n}\right), \bar{h}_{l j}^{(N P L, n)}\left(\frac{l}{n}\right), \max _{r=1, \cdots, m} \bar{h}_{l j}^{(N P L, n)}\left(t_{r}^{*}\right)\right\}, & \text { if } Z_{l j}^{(N P L, n)} \neq \varnothing \\
\max \left\{\bar{h}_{l j}^{(N P L, n)}\left(\frac{l-1}{n}\right), \bar{h}_{l j}^{(N P L, n)}\left(\frac{l}{n}\right)\right\}, & \text { if } Z_{l j}^{(N P L, n)}=\varnothing .\end{cases}
\end{aligned}
$$

Let $Z_{l j}^{(P N U, n)}$ denote the set of all zeros of the real-valued function $\frac{d}{d t}\left(\breve{h}_{l j}^{(N P, n)}(t)+a_{j}^{U}(t)\right)$ for $j \in N$. Then we can similarly obtain

$$
\begin{aligned}
\max _{t \in \bar{E}_{l}^{(n)}} \bar{h}_{l j}^{(P N U, n)}(t) \\
\quad= \begin{cases}\max \left\{\bar{h}_{l j}^{(P N U, n)}\left(\frac{l-1}{n}\right), \bar{h}_{l j}^{(P N U, n)}\left(\frac{l}{n}\right), \max _{r=1, \cdots, m} \bar{h}_{l j}^{(P N U, n)}\left(t_{r}^{*}\right)\right\}, & \text { if } Z_{l j}^{(P N U, n)} \neq \varnothing \\
\max \left\{\bar{h}_{l j}^{(P N U, n)}\left(\frac{l-1}{n}\right), \bar{h}_{l j}^{(P N U, n)}\left(\frac{l}{n}\right)\right\}, & \text { if } Z_{l j}^{(P N U, n)}=\varnothing .\end{cases}
\end{aligned}
$$

Therefore, using (214), (215), (221) and (222), we can obtain the desired supremum (209), and using (216), (217), (223) and (224), we can obtain the desired supremum (210).

Now we consider the following cases.

- Suppose that $\bar{h}_{l j}^{(P N L, n)}, \bar{h}_{l j}^{(N P U, n)}, \bar{h}_{l j}^{(N P L, n)}$ and $\bar{h}_{l j}^{(P N U, n)}$ are linear functions of $t$ on $\bar{E}_{l}^{(n)}$. This situation will happen when the fuzzy numbers are taken to be the triangular fuzzy numbers. We also remark that the triangular fuzzy numbers are frequently used in the real problems. Now the linear functions are assumed to be the following forms:

$$
\begin{aligned}
& \bar{h}_{l j}^{(P N L, n)}(t)=h_{l j}^{(P N, n)}(t)+a_{j}^{L}(t)=\mathfrak{r}_{l j}^{(P N L, n)} \cdot t+\mathfrak{s}_{l j}^{(P N L, n)} \text { for } j \in P \\
& \bar{h}_{l j}^{(N P U, n)}(t)=h_{l j}^{(N P, n)}(t)+a_{j}^{U}(t)=\mathfrak{r}_{l j}^{(N P U, n)} \cdot t+\mathfrak{s}_{l j}^{(N P U, n)} \text { for } j \in P
\end{aligned}
$$




$$
\begin{gathered}
\bar{h}_{l j}^{(N P L, n)}(t)=h_{l j}^{(N P, n)}(t)+a_{j}^{L}(t)=\mathfrak{r}_{l j}^{(N P L, n)} \cdot t+\mathfrak{s}_{l j}^{(N P L, n)} \text { for } j \in N \\
\bar{h}_{l j}^{(P N U, n)}(t)=h_{l j}^{(P N, n)}(t)+a_{j}^{U}(t)=\mathfrak{r}_{l j}^{(P N U, n)} \cdot t+\mathfrak{s}_{l j}^{(P N U, n)} \text { for } j \in N .
\end{gathered}
$$

Using (214)-(217), we obtain

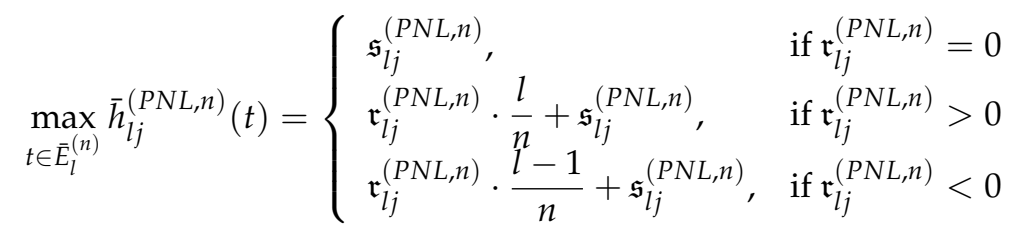

$$
\begin{aligned}
& \max _{t \in \bar{E}_{l}^{(n)}} \bar{h}_{l j}^{(N P U, n)}(t)=\left\{\begin{array}{cl}
\mathfrak{s}_{l j}^{(N P U, n),} & \text { if } \mathfrak{r}_{l j}^{(N P U, n)}=0 \\
\mathfrak{r}_{l j}^{(N P U, n)} \cdot \frac{l}{n}+\mathfrak{s}_{l j}^{(N P U, n),} & \text { if } \mathfrak{r}_{l j}^{(N P U, n)}>0 \\
\mathfrak{r}_{l j}^{(N P U, n)} \cdot \frac{l-1}{n}+\mathfrak{s}_{l j}^{(N P U, n),}, & \text { if } \mathfrak{r}_{l j}^{(N P U, n)}<0
\end{array}\right. \\
& \max _{t \in \bar{E}_{l}^{(n)}} \bar{h}_{l j}^{(N P L, n)}(t)= \begin{cases}\mathfrak{s}_{l j}^{(N P L, n),} & \text { if } \mathfrak{r}_{l j}^{(N P L, n)}=0 \\
\mathfrak{r}_{l j}^{(N P L, n)} \cdot \frac{l}{n}+\mathfrak{s}_{l j}^{(N P L, n),} & \text { if } \mathfrak{r}_{l j}^{(N P L, n)}>0 \\
\mathfrak{r}_{l j}^{(N P L, n)} \cdot \frac{l-1}{n}+\mathfrak{s}_{l j}^{(N P L, n),}, & \text { if } \mathfrak{r}_{l j}^{(N P L, n)}<0\end{cases} \\
& \max _{t \in \bar{E}_{l}^{(n)}} \bar{h}_{l j}^{(P N U, n)}(t)=\left\{\begin{array}{cl}
\mathfrak{s}_{l j}^{(P N U, n),} & \text { if } \mathfrak{r}_{l j}^{(P N U, n)}=0 \\
\mathfrak{r}_{l j}^{(P N U, n)} \cdot \frac{l}{n}+\mathfrak{s}_{l j}^{(P N U, n),} & \text { if } \mathfrak{r}_{l j}^{(P N U, n)}>0 \\
\mathfrak{r}_{l j}^{(P N U, n)} \cdot \frac{l-1}{n}+\mathfrak{s}_{l j}^{(P N U, n),}, & \text { if } \mathfrak{r}_{l j}^{(P N U, n)}<0
\end{array}\right.
\end{aligned}
$$

- $\quad$ Suppose that $\bar{h}_{l j}^{(P N L, n)}, \bar{h}_{l j}^{(N P U, n)}, \bar{h}_{l j}^{(N P L, n)}$ and $\bar{h}_{l j}^{(P N U, n)}$ are not the linear functions of $t$ on $\bar{E}_{l}^{(n)}$. In order to obtain the zero $t^{*}$ of $\frac{d}{d t}\left(\breve{h}_{l j}^{(P N, n)}(t)+a_{j}^{L}(t)\right)$, we can apply the Newton's method to generate a sequence $\left\{t_{r}\right\}_{r=1}^{\infty}$ such that $t_{r} \rightarrow t^{*}$ as $r \rightarrow \infty$. The iteration is given by

$$
t_{r+1}=t_{r}-\frac{\left.\frac{d}{d t} \breve{h}_{l j}^{(P N, n)}(t)\right|_{t=t_{r}}+\left.\frac{d}{d t} a_{j}^{L}(t)\right|_{t=t_{r}}}{\left.\frac{d^{2}}{d t^{2}} \breve{h}_{l j}^{(P N, n)}(t)\right|_{t=t_{r}}+\left.\frac{d^{2}}{d t^{2}} a_{j}^{L}(t)\right|_{t=t_{r}}}
$$

for $r=0,1,2, \cdots$. The initial guess is $t_{0}$. Since the real-valued function $\frac{d}{d t}\left(\breve{h}_{l j}^{(P N, n)}(t)+a_{j}^{L}(t)\right)$ may have more than one zero, we need to apply the Newton's method by taking as many as possible for the initial guesses $t_{0}$ 's. The zeros of

$$
\frac{d}{d t}\left(\breve{h}_{l j}^{(P N, n)}(t)+a_{j}^{U}(t)\right), \quad \frac{d}{d t}\left(\breve{h}_{l j}^{(N P, n)}(t)+a_{j}^{L}(t)\right) \text { and } \frac{d}{d t}\left(\breve{h}_{l j}^{(N P, n)}(t)+a_{j}^{U}(t)\right)
$$

can be similarly obtained.

The computational procedure is given below.

- Step 1. Set the error tolerance $\epsilon$ and the initial value of natural number $n \in \mathbb{N}$.

- $\quad$ Step 2. Find the optimal objective value $V\left(\mathrm{D}_{n}\right)$ and optimal solution $\mathbf{w}^{(* n)}$ of dual problem $\left(\mathrm{D}_{n}\right)$.

- Step 3. Find the sets $Z_{l j}^{(P N L, n)}, Z_{l j}^{(N P U, n)}, Z_{l j}^{(N P L, n)}$ and $Z_{l j}^{(P N U, n)}$ of all zeros of the real-valued functions $\frac{d}{d t}\left(\breve{h}_{l j}^{(P N, n)}(t)+a_{j}^{L}(t)\right), \frac{d}{d t}\left(\breve{h}_{l j}^{(N P, n)}(t)+a_{j}^{U}(t), \frac{d}{d t}\left(\breve{h}_{l j}^{(N P, n)}(t)+a_{j}^{L}(t)\right)\right.$ and $\frac{d}{d t}\left(\breve{h}_{l j}^{(P N, n)}(t)+\right.$ $\left.a_{j}^{U}(t)\right)$, respectively, by applying the Newton method given in (229). 
- Step 4. Evaluate the supremum (209) and (210) according to (214)-(217) and (221)-(224), respectively.

- Step 5. Obtain the real numbers $\pi_{l}^{(P N, n)}$ and $\pi_{l}^{(N P, n)}$ according to (76) by using the supremum obtained in step 4 .

- Step 6. Evaluate the error estimation $\varepsilon_{n}$ according to (106). If $\varepsilon_{n}<\epsilon$, then go to step 7; otherwise, consider one more subdivision by setting $n \leftarrow n+1$ and go to step 2 .

- $\quad$ Step 7. Find the optimal solution $\mathbf{z}^{(* n)}$ of primal problem $\left(\mathrm{P}_{n}\right)$.

- Step 8. Set the step functions $\mathbf{x}^{(n)}=\left(\mathbf{x}^{(L, n)}, \mathbf{x}^{(U, n)}\right)$ defined in (62) and (63), which will be the approximate solution of problem SOP. The actual error between the optimal objective value $V(\mathrm{SOP})$ and the objective value at $\mathbf{x}^{(n)}$ is less than $\varepsilon_{n}$ by Theorem 4 , where the error tolerance $\epsilon$ is reached for this partition $\mathcal{P}_{n}$. This approximate solution $\mathbf{x}^{(n)}$ is an $\epsilon$-optimal solution by referring to Theorem 5.

The above computational procedure obtains the real-valued functions $x_{j}^{(L, n)}$ and $x_{j}^{(U, n)}$ on $[0,1]$ that can form the $\alpha$-level closed intervals of a fuzzy number $\widetilde{x}_{j}^{(n)}$ for $j=1, \cdots, q$. More precisely, the $\alpha$-level sets of $\widetilde{x}_{j}^{(n)}$ is given by

$$
\left(\widetilde{x}_{j}^{(n)}\right)_{\alpha}=\left[\left(\widetilde{x}_{j}^{(n)}\right)_{\alpha}^{L},\left(\widetilde{x}_{j}^{(n)}\right)_{\alpha}^{U}\right]=\left[x_{j}^{(L, n)}(\alpha), x_{j}^{(U, n)}(\alpha)\right],
$$

for $\alpha \in[0,1]$. According to the decomposition theorem in fuzzy sets theory, the membership function $\xi_{\widetilde{x}_{j}^{(n)}}$ of $\widetilde{x}_{j}^{(n)}$ is given by

$$
\xi_{\widetilde{x}_{j}^{(n)}}(r)=\sup _{\alpha \in[0,1]} \alpha \cdot \chi_{\left(\widetilde{x}_{j}^{(n)}\right)_{\alpha}}(r),
$$

where $\chi_{A}$ denotes the indicator function of set $A$ given by

$$
\chi_{A}(r)= \begin{cases}1 & \text { if } r \in A \\ 0 & \text { if } r \notin A .\end{cases}
$$

According to Theorem 1, if $\widetilde{\mathbf{x}}$ is an $\epsilon$-optimal solution of problem SOP, then $\widetilde{\mathbf{x}}$ is called an $\epsilon$-nondominated optimal solution of problem FOP. Since $\mathbf{x}^{(n)}=\left(\mathbf{x}^{(L, n)}, \mathbf{x}^{(U, n)}\right)$ obtained in the above computational procedure is an $\epsilon$-optimal solution of problem SOP, i.e., the vector

$$
\widetilde{\mathbf{x}}^{(n)}=\left(\widetilde{x}_{1}^{(n)}, \widetilde{x}_{2}^{(n)}, \ldots, \widetilde{x}_{p}^{(n)}\right),
$$

of fuzzy numbers obtained from (230) is an $\epsilon$-optimal solution of problem SOP, it follows that $\widetilde{\mathbf{x}}^{(n)}$ is an $\epsilon$-nondominated optimal solution of problem FOP. The numerical examples will be provided below to demonstrate the $\epsilon$-nondominated optimal solution.

\section{Numerical Examples}

Now we shall present some numerical examples to demonstrate the usefulness of the numerical technique proposed in this paper. We calculated the error $\varepsilon_{n}$ by separately considering the bell-shaped fuzzy numbers and triangular fuzzy numbers. 


\subsection{Using the Bell-Shaped Fuzzy Numbers}

We considered the bell-shaped fuzzy number $\widetilde{a}=\left(L_{\widetilde{a}}, \breve{a}^{L}, \breve{a}^{\circ L}, \breve{a}^{\circ U}, \breve{a}^{U}, R_{\widetilde{a}}\right)$ with the membership functions defined by

$$
\xi_{\widetilde{a}}(r)= \begin{cases}L_{\widetilde{a}}(r) & \text { if } \breve{a}^{L} \leq r<\breve{a}^{\circ L} \\ 1 & \text { if } \breve{a}^{\circ L} \leq r \leq \breve{a}^{\circ U} \\ R_{\widetilde{a}}(r) & \text { if } \breve{a}^{\circ U}<r \leq \breve{a}^{U} \\ 0 & \text { otherwise, }\end{cases}
$$

where $L_{\widetilde{a}}$ is a strictly increasing function define on $\left[\breve{a}^{L}, \breve{a}^{\circ L}\right)$ into $[0,1]$ and $R_{\widetilde{a}}$ is a strictly decreasing function define on $\left(\breve{a}^{\circ U}, \breve{a} U\right]$ into $[0,1]$. This kind of fuzzy number is also called an $L R$-fuzzy number (ref. Dubois and Prade [37]). If $\breve{a}^{\circ L}=\breve{a}^{\circ} U=\breve{a}$, we simply write $\widetilde{a}=\left(L_{\widetilde{a}}, \breve{a}^{L}, \breve{a}, \breve{a}^{U}, R_{\widetilde{a}}\right)$. If $\tilde{a}$ is assumed to be a nonnegative fuzzy number, then $\breve{a}^{L}>0$. If $\tilde{a}$ is assumed to be a nonpositive fuzzy number, then $\breve{a}^{U}<0$.

Since $L_{\widetilde{a}}$ and $R_{\widetilde{a}}$ are strictly monotonic functions, it follows that their inverse functions exist and are denoted by $L_{\widetilde{a}}^{-1}$ and $R_{\widetilde{a}}^{-1}$, respectively. Let

$$
L_{\widetilde{a}}\left(\breve{a}^{L}\right)=\beta^{L} \text { and } R_{\widetilde{a}}\left(\breve{a}^{U}\right)=\beta^{U} .
$$

We assume that the membership function $\xi_{\widetilde{a}}$ is continuous. Then the functions $L_{\widetilde{a}}$ and $R_{\widetilde{a}}$ are also continuous satisfying

$$
L_{\widetilde{a}}\left(\breve{a}^{\circ L}\right)=1=R_{\widetilde{a}}\left(\breve{a}^{\circ U}\right) .
$$

Then the $\alpha$-level set is given by the following cases.

- $\quad$ Suppose that $\beta^{L} \leq \beta^{U}$. Then

$$
\widetilde{a}_{\alpha}= \begin{cases}{\left[\breve{a}^{L}, \breve{a}^{U}\right],} & \text { if } 0 \leq \alpha \leq \beta^{L} \\ {\left[L_{\widetilde{a}}^{-1}(\alpha), \breve{a}^{U}\right],} & \text { if } \beta^{L}<\alpha \leq \beta^{U} \\ {\left[L_{\widetilde{a}}^{-1}(\alpha), R_{\widetilde{a}}^{-1}(\alpha)\right],} & \text { if } \beta^{U}<\alpha \leq 1 .\end{cases}
$$

For convenience, we define the real-valued functions $L_{\widetilde{a}}^{*}$ and $R_{\widetilde{a}}^{*}$ on $[0,1]$ as follows:

$$
L_{\widetilde{a}}^{*}(\alpha)=\left\{\begin{array}{ll}
\breve{a}^{L}, & \text { if } 0 \leq \alpha \leq \beta^{L} \\
L_{\widetilde{a}}^{-1}(\alpha), & \text { if } \beta^{L}<\alpha \leq 1
\end{array} \text { and } R_{\widetilde{a}}^{*}= \begin{cases}\breve{a}^{U}, & \text { if } 0 \leq \alpha \leq \beta^{U} \\
R_{\widetilde{a}}^{-1}(\alpha), & \text { if } \beta^{U}<\alpha \leq 1 .\end{cases}\right.
$$

We also see that

$$
\widetilde{a}_{\alpha}^{L}=L_{\widetilde{a}}^{*}(\alpha) \text { and } \widetilde{a}_{\alpha}^{U}=R_{\widetilde{a}}^{*}(\alpha)
$$

for all $\alpha \in[0,1]$.

- $\quad$ Suppose that $\beta^{L}>\beta^{U}$. Then

$$
\widetilde{a}_{\alpha}= \begin{cases}{\left[\breve{a}^{L}, \breve{a}^{U}\right],} & \text { if } 0 \leq \alpha \leq \beta^{U} \\ {\left[\breve{a}^{L}, R_{\widetilde{a}}^{-1}(\alpha)\right],} & \text { if } \beta^{U}<\alpha \leq \beta^{L} \\ {\left[L_{\widetilde{a}}^{-1}(\alpha), R_{\widetilde{a}}^{-1}(\alpha)\right],} & \text { if } \beta^{L}<\alpha \leq 1 .\end{cases}
$$

It is clear to see that

$$
\widetilde{a}_{\alpha}^{L}=L_{\widetilde{a}}^{*}(\alpha) \text { and } \widetilde{a}_{\alpha}^{U}=R_{\widetilde{a}}^{*}(\alpha)
$$

for all $\alpha \in[0,1]$ as defined in (232).

In other words, either $\beta^{L} \leq \beta^{U}$ or $\beta^{L}>\beta^{U}$, the end-points $\widetilde{a}_{\alpha}^{L}$ and $\widetilde{a}_{\alpha}^{U}$ always have the same forms. 
Assume that all of the fuzzy numbers in the fuzzy linear programming problem FLP are taken to be the bell-shaped fuzzy numbers given by

$$
\begin{gathered}
\widetilde{a}_{j}=\left(L_{\widetilde{a}_{j}}, \breve{a}_{j}^{L}, \breve{a}_{j}^{\circ L}, \breve{a}_{j}^{\circ U}, \breve{a}_{j}^{U}, R_{\widetilde{a}_{j}}\right) \text { for } j=1, \cdots, q \\
\widetilde{c}_{i}=\left(L_{\widetilde{c}_{i}}, \breve{c}_{i}^{L}, \breve{c}_{i}^{\circ L}, \breve{c}_{i}^{\circ U}, \breve{c}_{i}^{U}, R_{\widetilde{c}_{i}}\right) \text { for } i=1, \cdots, p \\
\widetilde{b}_{i j}=\left(L_{\widetilde{b}_{i j}} \breve{b}_{i j}^{L}, \breve{b}_{i j}^{\circ L}, \breve{b}_{i j}^{\circ U}, \breve{b}_{i j}^{U}, R_{\widetilde{b}_{i j}}\right) \text { for } i=1, \cdots, n \text { and } j=1, \cdots, q .
\end{gathered}
$$

Then we have the following real-valued functions defined on $[0,1]$ :

$$
\begin{array}{r}
a_{j}^{L}(t)=L_{\widetilde{a}_{j}}^{*}(t) \text { and } a_{j}^{U}(t)=R_{\widetilde{a}_{j}}^{*}(t) \\
c_{i}^{L}(t)=L_{\widetilde{c}_{i}}^{*}(t) \text { and } c_{i}^{U}(t)=R_{\widetilde{c}_{i}}^{*}(t) \\
b_{i j}^{L}(t)=L_{\vec{b}_{i j}}^{*}(t) \text { and } b_{i j}^{U}(t)=R_{\widetilde{b}_{i j}}^{*}(t) .
\end{array}
$$

For $l=1, \cdots, n$, from (81) and (82), we see that

$$
\mathfrak{b}_{l}^{(P N, n)}=\min _{j=1, \cdots, q}\left\{\sum_{\left\{i: j \in P_{i}\right\}} L_{\widetilde{b}_{i j}}^{*}\left(\frac{l-1}{n}\right)+\sum_{\left\{i: j \in N_{i}\right\}} R_{\widetilde{b}_{i j}}^{*}\left(\frac{l}{n}\right)\right\}
$$

and

$$
\mathfrak{b}_{l}^{(N P, n)}=\min _{j=1, \cdots, q}\left\{\sum_{\left\{i: j \in N_{i}\right\}} L_{\widetilde{b}_{i j}}^{*}\left(\frac{l-1}{n}\right)+\sum_{\left\{i: j \in P_{i}\right\}} R_{\widetilde{b}_{i j}}^{*}\left(\frac{l}{n}\right)\right\} .
$$

From (42)-(44), we have

$$
\begin{aligned}
& a_{l j}^{(L, n)}=L_{\widetilde{a}_{j}}^{*}\left(\frac{l-1}{n}\right) \text { and } a_{l j}^{(U, n)}=R_{\widetilde{a}_{j}}^{*}\left(\frac{l}{n}\right) \\
& c_{l i}^{(L, n)}=L_{\widetilde{c}_{i}}^{*}\left(\frac{l-1}{n}\right) \text { and } c_{l i}^{(U, n)}=R_{\widetilde{c}_{i}}^{*}\left(\frac{l}{n}\right) \\
& b_{l i j}^{(L, n)}=L_{\widetilde{b}_{i j}}^{*}\left(\frac{l}{n}\right) \text { and } b_{l i j}^{(U, n)}=R_{\widetilde{b}_{i j}}^{*}\left(\frac{l-1}{n}\right) .
\end{aligned}
$$

In order to evaluate $\pi_{l}^{(P N, n)}$ and $\pi_{l}^{(N P, n)}$ presented in (76), we need to solve (214)-(217). From (213), we are going to calculate

$$
\begin{aligned}
& \widehat{h}_{l j}^{(P N, n)}=\sum_{\left\{i: j \in P_{i}\right\}} L_{\widetilde{b}_{i j}}^{*}\left(\frac{l}{n}\right) \cdot w_{l i}^{(* I, n)}+\sum_{\left\{i: j \in N_{i}\right\}} R_{\widetilde{b}_{i j}}^{*}\left(\frac{l-1}{n}\right) \cdot w_{l i}^{(* I I, n)}(\operatorname{using}(238)) \\
& \widehat{h}_{l j}^{(N P, n)}=\sum_{\left\{i: j \in N_{i}\right\}} L_{\widetilde{b}_{i j}}^{*}\left(\frac{l}{n}\right) \cdot w_{l i}^{(* I, n)}+\sum_{\left\{i: j \in P_{i}\right\}} R_{\widetilde{b}_{i j}}^{*}\left(\frac{l-1}{n}\right) \cdot w_{l i}^{(* I I, n)} \\
& \breve{h}_{l j}^{(P N, n)}(t)=-\sum_{\left\{i: j \in P_{i}\right\}} L_{\breve{b}_{i j}}^{*}(t) \cdot w_{l i}^{(* I, n)}-\sum_{\left\{i: j \in N_{i}\right\}} R_{\breve{b}_{i j}}^{*}(t) \cdot w_{l i}^{(* I I, n)}(\operatorname{using}(235)) \\
& \breve{h}_{l j}^{(N P, n)}(t)=-\sum_{\left\{i: j \in N_{i}\right\}} L_{\breve{b}_{i j}}^{*}(t) \cdot w_{l i}^{(* I, n)}-\sum_{\left\{i: j \in P_{i}\right\}} R_{\breve{b}_{i j}}^{*}(t) \cdot w_{l i}^{(* I I, n)} .
\end{aligned}
$$

Using (218), (219) and (213), we also have

$$
\bar{h}_{l j}^{(P N L, n)}(t)=h_{l j}^{(P N, n)}(t)+a_{j}^{L}(t)=\widehat{h}_{l j}^{(P N, n)}+\breve{h}_{l j}^{(P N, n)}(t)+L_{\widetilde{a}_{j}}^{*}(t) \text { for } j \in P
$$




$$
\begin{aligned}
& \bar{h}_{l j}^{(N P U, n)}(t)=h_{l j}^{(N P, n)}(t)+a_{j}^{U}(t)=\widehat{h}_{l j}^{(N P, n)}+\breve{h}_{l j}^{(N P, n)}(t)+R_{\widetilde{a}_{j}}^{*}(t) \text { for } j \in P \\
& \bar{h}_{l j}^{(N P L, n)}(t)=h_{l j}^{(N P, n)}(t)+a_{j}^{L}(t)=\widehat{h}_{l j}^{(N P, n)}+\breve{h}_{l j}^{(N P, n)}(t)+L_{\widetilde{a}_{j}}^{*}(t) \text { for } j \in N \\
& \bar{h}_{l j}^{(P N U, n)}(t)=h_{l j}^{(P N, n)}(t)+a_{j}^{U}(t)=\widehat{h}_{l j}^{(P N, n)}+\breve{h}_{l j}^{(P N, n)}(t)+R_{\widetilde{a}_{j}}^{*}(t) \text { for } j \in N
\end{aligned}
$$

Therefore, from (72)-(75), we obtain

$$
\begin{aligned}
& \pi_{l}^{(P N L, n)}=\max _{j \in P}\left\{-L_{\widetilde{a}_{j}}^{*}\left(\frac{l-1}{n}\right)+\max _{t \in \bar{E}_{l}^{(n)}} \bar{h}_{l j}^{(P N L, n)}(t)\right\} \\
& \pi_{l}^{(P N U, n)}=\max _{j \in N}\left\{-R_{\widetilde{a}_{j}}^{*}\left(\frac{l}{n}\right)+\max _{t \in \bar{E}_{l}^{(n)}} \bar{h}_{l j}^{(P N U, n)}(t)\right\} \\
& \pi_{l}^{(N P L, n)}=\max _{j \in N}\left\{-L_{\widetilde{a}_{j}}^{*}\left(\frac{l-1}{n}\right)+\max _{t \in \bar{E}_{l}^{(n)}} \bar{h}_{l j}^{(N P L, n)}(t)\right\} \\
& \pi_{l}^{(N P U, n)}=\max _{j \in P}\left\{-R_{\widetilde{a}_{j}}^{*}\left(\frac{l}{n}\right)+\max _{t \in \bar{E}_{l}^{(n)}} \bar{h}_{l j}^{(N P U, n)}(t)\right\},
\end{aligned}
$$

which can be calculated according to (229).

From (106) and (234), the error $\varepsilon_{n}$ between the approximate objective value and the optimal objective value is given by

$$
\begin{aligned}
\varepsilon_{n}= & -V\left(\mathrm{D}_{n}\right)+\sum_{l=1}^{n} \sum_{i=1}^{p}\left[\left(w_{l i}^{(* I, n)}+\max \left\{\frac{\pi_{l}^{(P N, n)}}{\mathfrak{b}_{l}^{(P N, n)},} \frac{\pi_{l}^{(N P, n)}}{\mathfrak{b}_{l}^{(N P, n)}}\right\}\right) \cdot \int_{\frac{l-1}{n}}^{\frac{l}{n}} L_{\widetilde{c}_{i}}^{*}(t) d t\right] \\
& +\sum_{l=1}^{n} \sum_{i=1}^{p}\left[\left(w_{l i}^{(* I I, n)}+\max \left\{\frac{\pi_{l}^{(P N, n)}}{\mathfrak{b}_{l}^{(P N, n)}}, \frac{\pi_{l}^{(N P, n)}}{\mathfrak{b}_{l}^{(N P, n)}}\right\}\right) \cdot \int_{\frac{l-1}{n}}^{\frac{l}{n}} R_{\widetilde{c}_{i}}^{*}(t) d t\right],
\end{aligned}
$$

where

$$
\pi_{l}^{(P N, n)}=\max \left\{\pi_{l}^{(P N L, n)}, \pi_{l}^{(P N U, n)}\right\} \text { and } \pi_{l}^{(N P, n)}=\max \left\{\pi_{l}^{(N P L, n)}, \pi_{l}^{(N P U, n)}\right\} .
$$

Next we provide a numerical example that assumes each fuzzy number to be the bell-shaped fuzzy number.

Example 1. We are gong to solve the following fuzzy linear programming problem

$$
\begin{array}{ll}
\max & \left(\widetilde{2} \otimes \widetilde{x}_{1}\right) \oplus\left(\widetilde{1} \otimes \widetilde{x}_{2}\right) \oplus\left(\widetilde{5} \otimes \widetilde{x}_{3}\right) \oplus\left(\widetilde{-3} \otimes \widetilde{x}_{4}\right) \\
\text { subject to } & \left(\widetilde{1} \otimes \widetilde{x}_{1}\right) \oplus\left(\widetilde{2} \otimes \widetilde{x}_{2}\right) \oplus\left(\widetilde{4} \otimes \widetilde{x}_{3}\right) \oplus\left(\widetilde{-1} \otimes \widetilde{x}_{4}\right) \preceq \widetilde{6} \\
& \left(\widetilde{2} \otimes \widetilde{x}_{1}\right) \oplus\left(\widetilde{3} \otimes \widetilde{x}_{2}\right) \oplus\left(\widetilde{-1} \otimes \widetilde{x}_{3}\right) \oplus\left(\widetilde{1} \otimes \widetilde{x}_{4}\right) \preceq \widetilde{12} \\
& \left(\widetilde{1} \otimes \widetilde{x}_{1}\right) \oplus\left(\widetilde{1} \otimes \widetilde{x}_{3}\right) \oplus\left(\widetilde{1} \otimes \widetilde{x}_{4}\right) \preceq \widetilde{4} \\
& \widetilde{x}_{1}, \widetilde{x}_{2}, \widetilde{x}_{3}, \widetilde{x}_{4} \text { are nonnegative fuzzy numbers. }
\end{array}
$$

The bell-shaped fuzzy number $\widetilde{a}$ is taken to be the form of

$$
\widetilde{a}=\left(e^{x-\breve{a}}, \breve{a}^{L}, \breve{a}, \breve{a}^{U}, e^{\breve{a}-x}\right),
$$


where the exponential function $e^{x-\breve{a}}$ is defined on $\left[\breve{a}^{L}, \breve{a}\right]$ and the exponential function $e^{\breve{a}-x}$ is defined on $\left[\breve{a}, \breve{a}^{U}\right]$. Then, from (231), we have

$$
\beta^{L}=e^{\breve{a}^{L}-\breve{a}} \text { and } \beta^{U}=e^{\breve{a}-\breve{a}^{U}} \text {. }
$$

According to (232), we have

$$
L_{\breve{a}}^{*}(t)= \begin{cases}\breve{a}^{L}, & \text { if } 0 \leq t \leq e^{\breve{a}^{L}-\breve{a}} \\ \breve{a}+\ln t, & \text { if } e^{\breve{a}^{L}-\breve{a}}<t \leq 1\end{cases}
$$

and

$$
R_{\breve{a}}^{*}(t)= \begin{cases}\breve{a}^{U}, & \text { if } 0 \leq t \leq e^{\breve{a}-\breve{a}^{u}} \\ \breve{a}-\ln t, & \text { if } e^{\breve{a}-\breve{a}^{U}}<t \leq 1 .\end{cases}
$$

The bell-shaped fuzzy numbers in this example are taken as follows:

$$
\begin{array}{lll}
\widetilde{1}=\left(e^{x-1}, 0.8,1,1.2, e^{1-x}\right), & \widetilde{2}=\left(e^{x-2}, 1.5,2,2.5, e^{2-x}\right), & \widetilde{3}=\left(e^{x-3}, 2,3,4, e^{3-x}\right) \\
\widetilde{4}=\left(e^{x-4}, 3,4,5, e^{4-x}\right), & \widetilde{5}=\left(e^{x-5}, 4,5,6, e^{5-x}\right), & \widetilde{6}=\left(e^{x-6}, 4,6,8, e^{6-x}\right) \\
\widetilde{12}=\left(e^{x-12}, 9,12,15, e^{12-x}\right), & \widetilde{-1}=\left(e^{x+1},-1.2,-1,-0.8, e^{-1-x}\right), & \widetilde{-3}=\left(e^{x+3},-4,-3,-2, e^{-3-x}\right) .
\end{array}
$$

Therefore the fuzzy data are given by

$$
\widetilde{a}_{1}=\widetilde{2}, \quad \widetilde{a}_{2}=\widetilde{1}, \quad \widetilde{a}_{3}=\widetilde{5}, \quad \widetilde{a}_{4}=\widetilde{-3}
$$

and

$$
\widetilde{c}_{1}=\widetilde{6}, \quad \widetilde{c_{2}}=\widetilde{12}, \quad \widetilde{c_{3}}=\widetilde{4}
$$

and

$$
\left[\begin{array}{llll}
\widetilde{b}_{11} & \widetilde{b}_{12} & \widetilde{b}_{13} & \widetilde{b}_{14} \\
\widetilde{b}_{21} & \widetilde{b}_{22} & \widetilde{b}_{23} & \widetilde{b}_{24} \\
\widetilde{b}_{31} & \widetilde{b}_{32} & \widetilde{b}_{33} & \widetilde{b}_{34}
\end{array}\right]=\left[\begin{array}{cccc}
\widetilde{1} & \widetilde{2} & \widetilde{4} & \widetilde{-1} \\
\widetilde{2} & \widetilde{3} & \widetilde{-1} & \widetilde{1} \\
\widetilde{1} & \widetilde{0} & \widetilde{1} & \widetilde{1}
\end{array}\right] .
$$

We also write

$$
\begin{aligned}
\widetilde{a}_{j} & =\left(e^{x-\breve{a}_{j}}, \breve{a}_{j}^{L}, \breve{a}_{j}, \breve{a}_{j}^{U}, e^{\breve{a}_{j}-x}\right) \text { for } j=1,2,3,4 \\
\widetilde{c}_{i} & =\left(e^{x-\breve{c}_{i}}, \breve{c}_{i}^{L}, \breve{c}_{i}, \breve{c}_{i}^{U}, e^{\breve{c}_{i}-x}\right) \text { for } i=1,2,3 \\
\widetilde{b}_{i j} & =\left(e^{x-\breve{b}_{i j}}, \breve{b}_{i j}^{L}, \breve{b}_{i j}, \breve{b}_{i j}^{U}, e^{\breve{b}_{i j} 0 x}\right) \text { for } i=1,2,3 \text { and } j=1,2,3,4 .
\end{aligned}
$$

Then, regarding $\widetilde{a}_{j}$, we have $P=\{1,2,3\}$ and $N=\{4\}$. Regarding $\widetilde{b}_{i j}$, we also have

$$
\begin{gathered}
P_{1}=\{1,2,3\} \text { and } N_{1}=\{4\} \\
P_{2}=\{1,2,4\} \text { and } N_{2}=\{3\} \\
P_{3}=\{1,2,3,4\} \text { and } N_{3}=\varnothing .
\end{gathered}
$$

It is clear to see that $\sigma^{P N}=0.6$ and $\sigma^{N P}=0.8$ in (17) and (18), respectively. From (236) and (237), we have

$$
\begin{aligned}
& \mathfrak{b}_{l}^{(P N, n)}=\min \left\{L_{\widetilde{1}}^{*}\left(\frac{l-1}{n}\right)+L_{\widetilde{2}}^{*}\left(\frac{l-1}{n}\right)+L_{\widetilde{1}}^{*}\left(\frac{l-1}{n}\right), L_{\widetilde{2}}^{*}\left(\frac{l-1}{n}\right)+L_{\widetilde{3}}^{*}\left(\frac{l-1}{n}\right),\right. \\
&\left.L_{\widetilde{4}}^{*}\left(\frac{l-1}{n}\right)+L_{\mathfrak{1}}^{*}\left(\frac{l-1}{n}\right)+R_{-1}^{*}\left(\frac{l}{n}\right), L_{1}^{*}\left(\frac{l-1}{n}\right)+L_{1}^{*}\left(\frac{l-1}{n}\right)+R_{-1}^{*}\left(\frac{l}{n}\right)\right\}
\end{aligned}
$$


and

$$
\begin{aligned}
\mathfrak{b}_{l}^{(N P, n)}=\min \left\{R_{\widetilde{1}}^{*}\left(\frac{l}{n}\right)+R_{\widetilde{2}}^{*}\left(\frac{l}{n}\right)+R_{\widetilde{1}}^{*}\left(\frac{l}{n}\right), R_{\widetilde{2}}^{*}\left(\frac{l}{n}\right)+R_{\widetilde{3}}^{*}\left(\frac{l}{n}\right),\right. \\
\left.R_{\widetilde{4}}^{*}\left(\frac{l}{n}\right)+R_{\tilde{1}}^{*}\left(\frac{l}{n}\right)+L_{-1}^{*}\left(\frac{l-1}{n}\right), R_{\widetilde{1}}^{*}\left(\frac{l}{n}\right)+R_{\widetilde{1}}^{*}\left(\frac{l}{n}\right)+L_{-1}^{*}\left(\frac{l-1}{n}\right)\right\} .
\end{aligned}
$$

From (239) and (242), we have

$$
\begin{aligned}
& \bar{h}_{l 1}^{(P N L, n)}(t)=\widehat{h}_{l 1}^{(P N, n)}+\breve{h}_{l 1}^{(P N, n)}(t)+L_{\widetilde{a}_{1}}^{*}(t) \\
&=\sum_{i=1}^{3} L_{\widetilde{b}_{i 1}}^{*}\left(\frac{l}{n}\right) \cdot w_{l i}^{(* I, n)}-\sum_{i=1}^{3} L_{\widetilde{b}_{i 1}}^{*}(t) \cdot w_{l i}^{(* I, n)}+L_{\widetilde{a}_{1}}^{*}(t) \\
&=L_{\overline{1}}^{*}\left(\frac{l}{n}\right) \cdot w_{l 1}^{(* I, n)}+L_{2}^{*}\left(\frac{l}{n}\right) \cdot w_{l 2}^{(* I, n)}+L_{\tilde{1}}^{*}\left(\frac{l}{n}\right) \cdot w_{l 3}^{(* I, n)} \\
&+ \begin{cases}-0.8 w_{l 1}^{(* I, n)}-1.5 w_{l 2}^{(* I, n)}-0.8 w_{l 3}^{(* I, n)}+1.5, & \text { if } 0 \leq t \leq e^{-0.5} \\
-0.8 w_{l 1}^{(* I, n)}-(2+\ln t) \cdot w_{l 2}^{(* I, n)}-0.8 w_{l 3}^{(* I, n)}+2+\ln t, & \text { if } 0<e^{-0.5}<t \leq e^{-0.2} \\
-w_{l 1}^{(* I, n)}-2 w_{l 2}^{(* I, n)}-w_{l 3}^{(* I, n)}+2 & \text { if } e^{-0.2}<t<1 \\
\quad+\ln t \cdot\left(-w_{l 1}^{(* I, n)}-w_{l 2}^{(* I, n)}-w_{l 3}^{(* I, n)}+1\right), & \end{cases}
\end{aligned}
$$

and

$$
\begin{aligned}
& \bar{h}_{l 2}^{(P N L, n)}(t)=\widehat{h}_{l 2}^{(P N, n)}+\breve{h}_{l 2}^{(P N, n)}(t)+L_{\widetilde{a}_{2}}^{*}(t) \\
&=\sum_{i=1}^{3} L_{\widetilde{b}_{i 2}}^{*}\left(\frac{l}{n}\right) \cdot w_{l i}^{(* I, n)}-\sum_{i=1}^{3} L_{\widetilde{b}_{i 2}}^{*}(t) \cdot w_{l i}^{(* I, n)}+L_{\widetilde{a}_{2}}^{*}(t) \\
&=L_{\widetilde{2}}^{*}\left(\frac{l}{n}\right) \cdot w_{l 1}^{(* I, n)}+L_{\widetilde{3}}^{*}\left(\frac{l}{n}\right) \cdot w_{l 2}^{(* I, n)} \\
&+ \begin{cases}-1.5 w_{l 1}^{(* I, n)}-2 w_{l 2}^{(* I, n)}+0.8, & \text { if } 0 \leq t \leq e^{-1} \\
-1.5 w_{l 1}^{(* I, n)}-(3+\ln t) \cdot w_{l 2}^{(* I, n)}+0.8, & \text { if } 0<e^{-1}<t \leq e^{-0.5} \\
-(1+\ln t) \cdot w_{l 1}^{(* I, n)}-(3+\ln t) \cdot w_{l 2}^{(* I, n)}+0.8, & \text { if } 0<e^{-0.5}<t \leq e^{-0.2} \\
-2 w_{l 1}^{(* I, n)}-3 w_{l 2}^{(* I, n)}+1 & \text { if } e^{-0.2}<t<1 \\
+\ln t \cdot\left(-w_{l 1}^{(* I, n)}-w_{l 2}^{(* I, n)}+1\right) & \end{cases}
\end{aligned}
$$

and

$$
\begin{aligned}
& \bar{h}_{l 3}^{(P N L, n)}(t)=\widehat{h}_{l 3}^{(P N, n)}+\breve{h}_{l 3}^{(P N, n)}(t)+L_{\widetilde{a}_{3}}^{*}(t) \\
&= L_{\breve{b}_{13}}^{*}\left(\frac{l}{n}\right) \cdot w_{l 1}^{(* I, n)}+L_{\breve{b}_{33}}^{*}\left(\frac{l}{n}\right) \cdot w_{l 3}^{(* I, n)}+R_{\breve{b}_{23}}^{*}\left(\frac{l-1}{n}\right) \cdot w_{l 2}^{(* I I, n)} \\
&-L_{\widetilde{b}_{13}}^{*}(t) \cdot w_{l 1}^{(* I, n)}-L_{\widetilde{b}_{33}}^{*}(t) \cdot w_{l 3}^{(* I, n)}-R_{\widetilde{b}_{23}}^{*}(t) \cdot w_{l 2}^{(* I I, n)}+L_{\mathfrak{a}_{3}}^{*}(t) \\
&= L_{\breve{4}}^{*}\left(\frac{l}{n}\right) \cdot w_{l 1}^{(* I, n)}+L_{\breve{1}}^{*}\left(\frac{l}{n}\right) \cdot w_{l 3}^{(* I, n)}+R_{-1}^{*}\left(\frac{l-1}{n}\right) \cdot w_{l 2}^{(* I I, n)} \\
&+ \begin{cases}-3 w_{l 1}^{(* I, n)}-0.8 w_{l 3}^{(* I, n)}+0.8 w_{l 2}^{(* I, n)}+4, & \text { if } 0 \leq t \leq e^{-1} \\
-(4+\ln t) \cdot w_{l 1}^{(* I, n)}-0.8 w_{l 3}^{(* I, n)}+0.8 w_{l 2}^{(* I I, n)}+5+\ln t, & \text { if } 0<e^{-1}<t \leq e^{-0.2} \\
-4 w_{l 1}^{(* I, n)}-w_{l 3}^{(* I, n)}+w_{l 2}^{(* I I, n)}+5 & \text { if } e^{-0.2}<t<1 \\
+\ln t \cdot\left(-w_{l 1}^{(* I, n)}-w_{l 3}^{(* I, n)}+w_{l 2}^{(* I I, n)}+1\right) & \end{cases}
\end{aligned}
$$


and

$$
\begin{aligned}
& \bar{h}_{l 4}^{(P N U, n)}(t)=\widehat{h}_{l 4}^{(P N, n)}+\breve{h}_{l 4}^{(P N, n)}(t)+R_{\widetilde{a}_{4}}^{*}(t) \\
& =L_{\widetilde{b}_{24}}^{*}\left(\frac{l}{n}\right) \cdot w_{l 2}^{(* I, n)}+L_{\widetilde{b}_{34}}^{*}\left(\frac{l}{n}\right) \cdot w_{l 3}^{(* I, n)}+R_{\widetilde{b}_{14}}^{*}\left(\frac{l-1}{n}\right) \cdot w_{l 1}^{(* I I, n)} \\
& -L_{\widetilde{b}_{24}}^{*}(t) \cdot w_{l 2}^{(* I, n)}-L_{\widetilde{b}_{34}}^{*}(t) \cdot w_{l 3}^{(* I, n)}-R_{\widetilde{b}_{14}}^{*}(t) \cdot w_{l 1}^{(* I I, n)}+R_{\widetilde{a}_{4}}^{*}(t) \\
& =L_{\widetilde{1}}^{*}\left(\frac{l}{n}\right) \cdot w_{l 2}^{(* I, n)}+L_{\widetilde{1}}^{*}\left(\frac{l}{n}\right) \cdot w_{l 3}^{(* I, n)}+R_{-1}^{*}\left(\frac{l-1}{n}\right) \cdot w_{l 1}^{(* I I, n)} \\
& + \begin{cases}-0.8 w_{l 2}^{(* I, n)}-0.8 w_{l 3}^{(* I, n)}+0.8 w_{l 1}^{(* I I, n)}-2, & \text { if } 0 \leq t \leq e^{-1} \\
-0.8 w_{l 2}^{(* I, n)}-0.8 w_{l 3}^{(* I, n)}+0.8 w_{l 1}^{(* I I, n)}-3-\ln t, & \text { if } 0<e^{-1}<t \leq e^{-0.2} \\
-w_{l 2}^{(* I, n)}-w_{l 3}^{(* I, n)}+w_{l 1}^{(* I I, n)}-3 & \text { if } e^{-0.2}<t<1 \\
\quad+\ln t \cdot\left(-w_{l 2}^{(* I, n)}-w_{l 3}^{(* I, n)}+w_{l 1}^{(* I I, n)}-1\right) & \end{cases}
\end{aligned}
$$

From (240) and (241), we also have

$$
\begin{aligned}
& \bar{h}_{l 1}^{(N P U, n)}(t)=\widehat{h}_{l 1}^{(N P, n)}+\breve{h}_{l 1}^{(N P, n)}(t)+R_{\widetilde{a}_{1}}^{*}(t) \\
& =\sum_{i=1}^{3} R_{\widetilde{b}_{i 1}}^{*}\left(\frac{l-1}{n}\right) \cdot w_{l i}^{(* I I, n)}-\sum_{i=1}^{3} R_{\widetilde{b}_{i 1}}^{*}(t) \cdot w_{l i}^{(* I I, n)}+R_{\widetilde{a}_{1}}^{*}(t) \\
& =R_{\widetilde{1}}^{*}\left(\frac{l-1}{n}\right) \cdot w_{l 1}^{(* I I, n)}+R_{2}^{*}\left(\frac{l-1}{n}\right) \cdot w_{l 2}^{(* I I, n)}+R_{\widetilde{1}}^{*}\left(\frac{l-1}{n}\right) \cdot w_{l 3}^{(* I I, n)} \\
& + \begin{cases}-1.2 w_{l 1}^{(* I I, n)}-2.5 w_{l 2}^{(* I I, n)}-1.2 w_{l 3}^{(* I I, n)}+2.5, & \text { if } 0 \leq t \leq e^{-0.5} \\
-1.2 w_{l 1}^{(* I I, n)}-(2-\ln t) \cdot w_{l 2}^{(* I I, n)}-1.2 w_{l 3}^{(* I, n)}+2-\ln t, & \text { if } 0<e^{-0.5}<t \leq e^{-0.2} \\
-w_{l 1}^{(* I, n)}-2 w_{l 2}^{(* I I, n)}-w_{l 3}^{(* I I, n)}+2 & \text { if } e^{-0.2}<t<1 \\
\quad+\ln t \cdot\left(w_{l 1}^{(* I I, n)}+w_{l 2}^{(* I I, n)}+w_{l 3}^{(* I I, n)}-1\right) & \end{cases}
\end{aligned}
$$

and

$$
\begin{aligned}
& \bar{h}_{l 2}^{(N P U, n)}(t)=\widehat{h}_{l 2}^{(N P, n)}+\breve{h}_{l 2}^{(N P, n)}(t)+R_{\widetilde{a}_{2}}^{*}(t) \\
& =\sum_{i=1}^{3} R_{\breve{b}_{i 2}}^{*}\left(\frac{l-1}{n}\right) \cdot w_{l i}^{(* I I, n)}-\sum_{i=1}^{3} R_{\widetilde{b}_{i 2}}^{*}(t) \cdot w_{l i}^{(* I I, n)}+R_{\widetilde{a}_{2}}^{*}(t) \\
& =R_{\widetilde{2}}^{*}\left(\frac{l-1}{n}\right) \cdot w_{l 1}^{(* I I, n)}+R_{\widetilde{3}}^{*}\left(\frac{l-1}{n}\right) \cdot w_{l 2}^{(* I I, n)} \\
& \quad+ \begin{cases}-2.5 w_{l 1}^{(* I I, n)}-4 w_{l 2}^{(* I I, n)}+1.2, & \text { if } 0 \leq t \leq e^{-1} \\
-2.5 w_{l 1}^{(* I I, n)}-(3-\ln t) \cdot w_{l 2}^{(* I I, n)}+1.2, & \text { if } 0<e^{-1}<t \leq e^{-0.5} \\
-(2-\ln t) \cdot w_{l 1}^{(* I I, n)}-(3-\ln t) \cdot w_{l 2}^{(* I I, n)}+1.2, & \text { if } 0<e^{-0.5}<t \leq e^{-0.2} \\
-2 w_{l 1}^{(* I I, n)}-3 w_{l 2}^{(* I I, n)}+1 & \text { if } e^{-0.2}<t<1 \\
+\ln t \cdot\left(w_{l 1}^{(* I I, n)}+w_{l 2}^{(* I I, n)}-1\right) & \end{cases}
\end{aligned}
$$


and

$$
\begin{aligned}
& \bar{h}_{l 3}^{(N P U, n)}(t)=\widehat{h}_{l 3}^{(N P, n)}+\breve{h}_{l 3}^{(N P, n)}(t)+R_{\widetilde{a}_{3}}^{*}(t) \\
&=R_{\vec{b}_{13}}^{*}\left(\frac{l-1}{n}\right) \cdot w_{l 1}^{(* I I, n)}+R_{\breve{b}_{33}}^{*}\left(\frac{l-1}{n}\right) \cdot w_{l 3}^{(* I I, n)}+L_{\breve{b}_{23}}^{*}\left(\frac{l}{n}\right) \cdot w_{l 2}^{(* I, n)} \\
& \quad-R_{\widetilde{b}_{13}}^{*}(t) \cdot w_{l 1}^{(* I I, n)}-R_{\breve{b}_{33}}^{*}(t) \cdot w_{l 3}^{(* I I, n)}-L_{\vec{b}_{23}}^{*}(t) \cdot w_{l 2}^{(* I, n)}+R_{\widetilde{a}_{3}}^{*}(t) \\
&= R_{\breve{4}}^{*}\left(\frac{l-1}{n}\right) \cdot w_{l 1}^{(* I I, n)}+R_{\breve{1}}^{*}\left(\frac{l-1}{n}\right) \cdot w_{l 3}^{(* I I, n)}+L_{-1}^{*}\left(\frac{l}{n}\right) \cdot w_{l 2}^{(* I, n)} \\
&+ \begin{cases}-5 w_{l 1}^{(* I I, n)}-1.2 w_{l 3}^{(* I I, n)}+1.2 w_{l 2}^{(* I, n)}+6, & \text { if } 0 \leq t \leq e^{-1} \\
-(4-\ln t) \cdot w_{l 1}^{(* I I, n)}-1.2 w_{l 3}^{(* I I, n)}+1.2 w_{l 2}^{(* I, n)}+5-\ln t, & \text { if } 0<e^{-1}<t \leq e^{-0.2} \\
-4 w_{l 1}^{(* I I, n)}-w_{l 3}^{(* I I, n)}+w_{l 2}^{(* I, n)}+5 & \text { if } e^{-0.2}<t<1 \\
+\ln t \cdot\left(w_{l 1}^{(* I I, n)}+w_{l 3}^{(* I I, n)}-w_{l 2}^{(* I, n)}-1\right) & \end{cases}
\end{aligned}
$$

and

$$
\begin{aligned}
& \bar{h}_{l 4}^{(N P L, n)}(t)=\widehat{h}_{l 4}^{(N P, n)}+\breve{h}_{l 4}^{(N P, n)}(t)+L_{\widetilde{a}_{4}}^{*}(t) \\
&=R_{\breve{b}_{24}}^{*}\left(\frac{l-1}{n}\right) \cdot w_{l 2}^{(* I I, n)}+R_{\breve{b}_{34}}^{*}\left(\frac{l-1}{n}\right) \cdot w_{l 3}^{(* I I, n)}+L_{\breve{b}_{14}}^{*}\left(\frac{l}{n}\right) \cdot w_{l 1}^{(* I, n)} \\
& \quad-R_{\widetilde{b}_{24}}^{*}(t) \cdot w_{l 2}^{(* I I, n)}-R_{\widetilde{b}_{34}}^{*}(t) \cdot w_{l 3}^{(* I I, n)}-L_{\widetilde{b}_{14}}^{*}(t) \cdot w_{l 1}^{(* I, n)}+L_{\widetilde{a}_{4}}^{*}(t) \\
&= R_{\breve{1}}^{*}\left(\frac{l-1}{n}\right) \cdot w_{l 2}^{(* I I, n)}+R_{\breve{1}}^{*}\left(\frac{l-1}{n}\right) \cdot w_{l 3}^{(* I I, n)}+L_{-1}^{*}\left(\frac{l}{n}\right) \cdot w_{l 1}^{(* I, n)} \\
&+ \begin{cases}-1.2 w_{l 2}^{(* I I, n)}-1.2 w_{l 3}^{(* I I, n)}+1.2 w_{l 1}^{(* I, n)}-4, & \text { if } 0 \leq t \leq e^{-1} \\
-1.2 \cdot w_{l 2}^{(* I I, n)}-1.2 w_{l 3}^{(* I I, n)}+1.2 w_{l 1}^{(* I, n)}-3+\ln t, & \text { if } 0<e^{-1}<t \leq e^{-0.2} \\
-w_{l 2}^{(* I I, n)}-w_{l 3}^{(* I I, n)}+w_{l 1}^{(* I, n)}-3 & \text { if } e^{-0.2}<t<1 \\
\quad+\ln t \cdot\left(w_{l 2}^{(* I I, n)}+w_{l 3}^{(* I I, n)}-w_{l 1}^{(* I, n)}+1\right) & \end{cases}
\end{aligned}
$$

Now we are going to calculate

$$
\max _{t \in \bar{E}_{l}^{(n)}} \bar{h}_{l j}^{(P N L, n)}(t), \quad \max _{t \in \bar{E}_{l}^{(n)}} \bar{h}_{l 4}^{(P N U, n)}(t), \quad \max _{t \in \bar{E}_{l}^{(n)}} \bar{h}_{l j}^{(N P U, n)}(t) \text { and } \max _{t \in \bar{E}_{l}^{(n)}} \bar{h}_{l 4}^{(N P L, n)}(t)
$$

for $j=1,2,3$. Let us recall that $\bar{E}_{l}^{(n)}=\left[\frac{l-1}{n}, \frac{l}{n}\right]$.

- To find the maximum of $\bar{h}_{l 1}^{(P N L, n)}$ on $\bar{E}_{l}^{(n)}$, let

$$
\begin{aligned}
\mathfrak{w}_{l 1}^{(1, n)} & =-0.8 w_{l 1}^{(* I, n)}-1.5 w_{l 2}^{(* I, n)}-0.8 w_{l 3}^{(* I, n)}+1.5 \\
\mathfrak{w}_{l 1}^{(2, n)} & =\ln \frac{l}{n} \cdot\left(1-w_{l 2}^{(* I, n)}\right)-0.8 w_{l 1}^{(* I, n)}-2 w_{l 2}^{(* I, n)}-0.8 w_{l 3}^{(* I, n)}+2 \\
\mathfrak{w}_{l 1}^{(3, n)} & =\ln \left(e^{-0.5}\right)\left(1-w_{l 2}^{(* I, n)}\right)-0.8 w_{l 1}^{(* I, n)}-2 w_{l 2}^{(* I, n)}-0.8 w_{l 3}^{(* I, n)}+2 \\
\mathfrak{w}_{l 1}^{(4, n)} & =\ln \frac{l-1}{n} \cdot\left(1-w_{l 2}^{(* I, n)}\right)-0.8 w_{l 1}^{(* I, n)}-2 w_{l 2}^{(* I, n)}-0.8 w_{l 3}^{(* I, n)}+2 \\
\mathfrak{w}_{l 1}^{(5, n)} & =\ln \left(e^{-0.2}\right)\left(1-w_{l 2}^{(* I, n)}\right)-0.8 w_{l 1}^{(* I, n)}-2 w_{l 2}^{(* I, n)}-0.8 w_{l 3}^{(* I, n)}+2 \\
\mathfrak{w}_{l 1}^{(6, n)} & =-w_{l 1}^{(* I, n)}-2 w_{l 2}^{(* I, n)}-w_{l 3}^{(* I, n)}+2 \\
\mathfrak{w}_{l 1}^{(7, n)} & =-w_{l 1}^{(* I, n)}-w_{l 2}^{(* I, n)}-w_{l 3}^{(* I, n)}+1 .
\end{aligned}
$$


In order to simplify the expressions for calculating the maximum on $\bar{E}_{l}^{(n)}$, we define the parametric function on $\bar{E}_{l}^{(n)}$ as follows. Given any fixed real numbers $a_{1}, a_{2}, a_{3}, a_{4}, a_{5}, a_{6}, a_{7}, w^{*}$ that are regarded as the parameters, for sufficiently large $n$ satisfying $\frac{1}{n}<1-e^{-0.2}$, we define a parametric function

$$
h_{1}\left[a_{1}, a_{2}, a_{3}, a_{4}, a_{5}, a_{6}, a_{7}, w^{*}\right](t): \bar{E}_{l}^{(n)} \rightarrow \mathbb{R}
$$

by

$$
\begin{aligned}
& h_{1}\left[a_{1}, a_{2}, a_{3}, a_{4}, a_{5}, a_{6}, a_{7}, w^{*}\right](t) \\
& \quad= \begin{cases}a_{1}, & \text { if } \frac{l}{n} \leq e^{-0.5} \\
\max \left\{a_{1}, a_{2}\right\}, & \text { if } \frac{l-1}{n} \leq e^{-0.5}<\frac{l}{n} \leq e^{-0.2}, w^{*} \leq 1 \\
\max \left\{a_{1}, a_{3}\right\}, & \text { if } \frac{l-1}{n} \leq e^{-0.5}<\frac{l}{n} \leq e^{-0.2}, w^{*}>1 \\
a_{2}, & \text { if } e^{-0.5}<\frac{l-1}{n}<\frac{l}{n} \leq e^{-0.2}, w^{*} \leq 1 \\
a_{4}, & \text { if } e^{-0.5}<\frac{l-1}{n}<\frac{l}{n} \leq e^{-0.2}, w^{*}>1 \\
\max \left\{a_{5}, a_{6}+\left(\ln \frac{l}{n}\right) \cdot a_{7}\right\}, & \text { if } e^{-0.5}<\frac{l-1}{n} \leq e^{-0.2}<\frac{l}{n}, w^{*} \leq 1, a_{7} \geq 0 \\
\max \left\{a_{5}, a_{6}+\ln \left(e^{-0.2}\right) \cdot a_{7}\right\}, & \text { if } e^{-0.5}<\frac{l-1}{n} \leq e^{-0.2}<\frac{l}{n}, w^{*} \leq 1, a_{7}<0 \\
\max \left\{a_{4}, a_{6}+\left(\ln \frac{l}{n}\right) \cdot a_{7}\right\}, & \text { if } e^{-0.5}<\frac{l-1}{n} \leq e^{-0.2}<\frac{l}{n}, w^{*}>1, a_{7} \geq 0 \\
\max \left\{a_{4}, a_{6}+\ln \left(e^{-0.2}\right) \cdot a_{7}\right\}, & \text { if } e^{-0.5}<\frac{l-1}{n} \leq e^{-0.2}<\frac{l}{n}, w^{*}>1, a_{7}<0 \\
a_{6}+\left(\ln \frac{l}{n}\right) \cdot a_{7}, & \text { if } e^{-0.2}<\frac{l-1}{n}, a_{7} \geq 0 \\
a_{6}+\left(\ln \frac{l-1}{n}\right) \cdot a_{7}, & \text { if } e^{-0.2}<\frac{l-1}{n}, a_{7}<0 .\end{cases}
\end{aligned}
$$

Then

$$
\begin{aligned}
\max _{t \in \bar{E}_{l}^{(n)}} \bar{h}_{l 1}^{(P N L, n)}(t)= & L_{\mathfrak{1}}^{*}\left(\frac{l}{n}\right) \cdot w_{l 1}^{(* I, n)}+L_{2}^{*}\left(\frac{l}{n}\right) \cdot w_{l 2}^{(* I, n)}+L_{\widetilde{1}}^{*}\left(\frac{l}{n}\right) \cdot w_{l 3}^{(* I, n)} \\
& +h_{1}\left[\mathfrak{w}_{l 1}^{(1, n)}, \mathfrak{w}_{l 1}^{(2, n)}, \mathfrak{w}_{l 1}^{(3, n)}, \mathfrak{w}_{l 1}^{(4, n)}, \mathfrak{w}_{l 1}^{(5, n)}, \mathfrak{w}_{l 1}^{(6, n)}, \mathfrak{w}_{l 1}^{(7, n)}, w_{l 2}^{(* I, n)}\right](t)
\end{aligned}
$$

- $\quad$ To find the maximum of $\bar{h}_{l 2}^{(P N L, n)}$ on $\bar{E}_{l}^{(n)}$, let

$$
\begin{aligned}
& \mathfrak{w}_{l 2}^{(1, n)}=-1.5 w_{l 1}^{(* I, n)}-2 w_{l 2}^{(* I, n)}+0.8 \\
& \mathfrak{w}_{l 2}^{(2, n)}=-1.5 w_{l 1}^{(* I, n)}-3 w_{l 2}^{(* I, n)}+0.8 \\
& \mathfrak{w}_{l 2}^{(3, n)}=-\ln \left(e^{-0.5}\right) \cdot\left(w_{l 1}^{(* I, n)}+w_{l 2}^{(* I, n)}\right)-w_{l 1}^{(* I, n)}-3 w_{l 2}^{(* I, n)}+0.8 \\
& \mathfrak{w}_{l 2}^{(4, n)}=-\ln \frac{l}{n} \cdot\left(w_{l 1}^{(* I, n)}+w_{l 2}^{(* I, n)}\right)-w_{l 1}^{(* I, n)}-3 w_{l 2}^{(* I, n)}+0.8 \\
& \mathfrak{w}_{l 2}^{(5, n)}=-\ln \frac{l-1}{n} \cdot\left(w_{l 1}^{(* I, n)}+w_{l 2}^{(* I, n)}\right)-w_{l 1}^{(* I, n)}-3 w_{l 2}^{(* I, n)}+0.8 \\
& \mathfrak{w}_{l 2}^{(6, n)}=-\ln \left(e^{-0.2}\right) \cdot\left(w_{l 1}^{(* I, n)}+w_{l 2}^{(* I, n)}\right)-w_{l 1}^{(* I, n)}-3 w_{l 2}^{(* I, n)}+0.8 \\
& \mathfrak{w}_{l 2}^{(7, n)}=-2 w_{l 1}^{(* I, n)}-3 w_{l 2}^{(* I, n)}+1 \\
& \mathfrak{w}_{l 2}^{(8, n)}=-w_{l 1}^{(* I, n)}-w_{l 2}^{(* I, n)}+1 .
\end{aligned}
$$

Given any fixed real numbers $a_{1}, a_{2}, a_{3}, a_{4}, a_{5}, a_{6}, a_{7}, a_{8}, w_{1}^{*}$, $w_{2}^{*}$ that are regarded as the parameters, for sufficiently large $n$ satisfying $\frac{1}{n}<1-e^{-0.2}$, we define a parametric function

$$
h_{2}\left[a_{1}, a_{2}, a_{3}, a_{4}, a_{5}, a_{6}, a_{7}, a_{8}, w_{1}^{*}, w_{2}^{*}\right](t): \bar{E}_{l}^{(n)} \rightarrow \mathbb{R}
$$


$b y$

$$
\begin{aligned}
& h_{2}\left[a_{1}, a_{2}, a_{3}, a_{4}, a_{5}, a_{6}, a_{7}, a_{8}, w_{1}^{*}, w_{2}^{*}\right](t) \\
& = \begin{cases}a_{1}, & \text { if } \frac{l}{n} \leq e^{-1} \\
\max \left\{a_{1}, a_{2}-\ln \left(e^{-1}\right) w_{2}^{*}\right\}, & \text { if } \frac{l-1}{n} \leq e^{-1}<\frac{l}{n} \leq e^{-0.5}, w_{2}^{*} \geq 0 \\
\max \left\{a_{1}, a_{2}-\left(\ln \frac{l}{n}\right) w_{2}^{*}\right\}, & \text { if } \frac{l-1}{n} \leq e^{-1}<\frac{l}{n} \leq e^{-0.5}, w_{2}^{*}<0 \\
a_{2}-\left(\ln \frac{l-1}{n}\right) w_{2}^{*}, & \text { if } e^{-1}<\frac{l-1}{n}<\frac{l}{n} \leq e^{-0.5}, w_{2}^{*} \geq 0 \\
a_{2}-\left(\ln \frac{l}{n}\right) w_{2}^{*}, & \text { if } e^{-1}<\frac{l-1}{n}<\frac{l}{n} \leq e^{-0.5}, w_{2}^{*}<0 \\
\max \left\{a_{2}-\left(\ln \frac{l-1}{n}\right) w_{2}^{*}, a_{3}\right\}, & \text { if } \frac{l-1}{n} \leq e^{-0.5}<\frac{l}{n} \leq e^{-0.2}, w_{2}^{*} \geq 0, w_{1}^{*}+w_{2}^{*} \geq 0 \\
\max \left\{a_{2}-\left(\ln \frac{l-1}{n}\right) w_{2}^{*}, a_{4}\right\}, & \text { if } \frac{l-1}{n} \leq e^{-0.5}<\frac{l}{n} \leq e^{-0.2}, w_{2}^{*} \geq 0, w_{1}^{*}+w_{2}^{*}<0 \\
\max \left\{a_{2}-\ln \left(e^{-0.5}\right) w_{2}^{*}, a_{3}\right\}, & \text { if } \frac{l-1}{n} \leq e^{-0.5}<\frac{l}{n} \leq e^{-0.2}, w_{2}^{*}<0, w_{1}^{*}+w_{2}^{*} \geq 0 \\
\max \left\{a_{2}-\ln \left(e^{-0.5}\right) w_{2}^{*}, a_{4}\right\}, & \text { if } \frac{l-1}{n} \leq e^{-0.5}<\frac{l}{n} \leq e^{-0.2}, w_{2}^{*}<0, w_{1}^{*}+w_{2}^{*}<0 \\
a_{5}, & \text { if } e^{-0.5}<\frac{l-1}{n}<\frac{l}{n} \leq e^{-0.2}, w_{1}^{*}+w_{2}^{*} \geq 0 \\
a_{4}, & \text { if } e^{-0.5}<\frac{l-1}{n}<\frac{l}{n} \leq e^{-0.2}, w_{1}^{*}+w_{2}^{*}<0 \\
\max \left\{a_{5}, a_{7}+\left(\ln \frac{l}{n}\right) a_{8}\right\}, & \text { if } e^{-0.5}<\frac{l-1}{n} \leq e^{-0.2}<\frac{l}{n}, w_{1}^{*}+w_{2}^{*} \geq 0, a_{8} \geq 0 \\
\max \left\{a_{5}, a_{7}+\ln \left(e^{-0.2}\right) a_{8}\right\}, & \text { if } e^{-0.5}<\frac{l-1}{n} \leq e^{-0.2}<\frac{l}{n}, w_{1}^{*}+w_{2}^{*} \geq 0, a_{8}<0 \\
\max \left\{a_{6}, a_{7}+\left(\ln \frac{l}{n}\right) a_{8}\right\}, & \text { if } e^{-0.5}<\frac{l-1}{n} \leq e^{-0.2}<\frac{l}{n}, w_{1}^{*}+w_{2}^{*}<0, a_{8} \geq 0 \\
\max \left\{a_{6}, a_{7}+\ln \left(e^{-0.2}\right) a_{8}\right\}, & \text { if } e^{-0.5}<\frac{l-1}{n} \leq e^{-0.2}<\frac{l}{n}, w_{1}^{*}+w_{2}^{*}<0, a_{8}<0 \\
a_{7}+\left(\ln \frac{l}{n}\right) a_{8}, & \text { if } e^{-0.2}<\frac{l-1}{n}, a_{8} \geq 0 \\
a_{7}+\left(\ln \frac{l-1}{n}\right) a_{8}, & \text { if } e^{-0.2}<\frac{l-1}{n}, a_{8}<0 .\end{cases}
\end{aligned}
$$

Then

$$
\begin{aligned}
& \max _{t \in \bar{E}_{l}^{(n)}} \bar{h}_{l 2}^{(P N, n)}(t)=L_{\widetilde{2}}^{*}\left(\frac{l}{n}\right) \cdot w_{l 1}^{(* I, n)}+L_{\widetilde{3}}^{*}\left(\frac{l}{n}\right) \cdot w_{l 2}^{(* I, n)} \\
& \quad+h_{2}\left[\mathfrak{w}_{l 2}^{(1, n)}, \mathfrak{w}_{l 2}^{(2, n)}, \mathfrak{w}_{l 2}^{(3, n)}, \mathfrak{w}_{l 2}^{(4, n)}, \mathfrak{w}_{l 2}^{(5, n)}, \mathfrak{w}_{l 2}^{(6, n)}, \mathfrak{w}_{l 2}^{(7, n)}, \mathfrak{w}_{l 2}^{(8, n)}, w_{l 1}^{(* I, n)}, w_{l 2}^{(* I, n)}\right](t)
\end{aligned}
$$

- To find the maximum of $\bar{h}_{l 3}^{(P N L, n)}$ on $\bar{E}_{l}^{(n)}$, let

$$
\begin{aligned}
& \mathfrak{w}_{l 3}^{(1, n)}=-3 w_{l 1}^{(* I, n)}-0.8 w_{l 3}^{(* I, n)}+0.8 w_{l 2}^{(* I I, n)}+4 \\
& \mathfrak{w}_{l 3}^{(2, n)}=\ln \frac{l}{n} \cdot\left(1-w_{l 1}^{(* I, n)}\right)-4 w_{l 1}^{(* I, n)}-0.8 w_{l 3}^{(* I, n)}+0.8 w_{l 2}^{(* I I, n)}+5 \\
& \mathfrak{w}_{l 3}^{(3, n)}=\ln \left(e^{-1}\right) \cdot\left(1-w_{l 1}^{(* I, n)}\right)-4 w_{l 1}^{(* I, n)}-0.8 w_{l 3}^{(* I, n)}+0.8 w_{l 2}^{(* I I, n)}+5 \\
& \mathfrak{w}_{l 3}^{(4, n)}=\ln \frac{l-1}{n} \cdot\left(1-w_{l 1}^{(* I, n)}\right)-4 w_{l 1}^{(* I, n)}-0.8 w_{l 3}^{(* I, n)}+0.8 w_{l 2}^{(* I I, n)}+5 \\
& \mathfrak{w}_{l 3}^{(5, n)}=\ln \left(e^{-0.2}\right) \cdot\left(1-w_{l 1}^{(* I, n)}\right)-4 w_{l 1}^{(* I, n)}-0.8 w_{l 3}^{(* I, n)}+0.8 w_{l 2}^{(* I I, n)}+5 \\
& \mathfrak{w}_{l 3}^{(6, n)}=-4 w_{l 1}^{(* I, n)}-w_{l 3}^{(* I, n)}+w_{l 2}^{(* I I, n)}+5 \\
& \mathfrak{w}_{l 3}^{(7, n)}=-w_{l 1}^{(* I, n)}-w_{l 3}^{(* I, n)}+w_{l 2}^{(* I I, n)}+1 .
\end{aligned}
$$

Given any fixed real numbers $a_{1}, a_{2}, a_{3}, a_{4}, a_{5}, a_{6}, a_{7}, w^{*}$ that are regarded as the parameters, for sufficiently large $n$ satisfying $\frac{1}{n}<1-e^{-0.2}$, we define a parametric function

$$
h_{3}\left[a_{1}, a_{2}, a_{3}, a_{4}, a_{5}, a_{6}, a_{7}, w^{*}\right](t): \bar{E}_{l}^{(n)} \rightarrow \mathbb{R}
$$


by

$$
\begin{aligned}
& h_{3}\left[a_{1}, a_{2}, a_{3}, a_{4}, a_{5}, a_{6}, a_{7}, w^{*}\right](t) \\
& \quad= \begin{cases}a_{1}, & \text { if } \frac{l}{n} \leq e^{-1} \\
\max \left\{a_{1}, a_{2}\right\}, & \text { if } \frac{l-1}{n} \leq e^{-1}<\frac{l}{n} \leq e^{-0.2}, w^{*} \leq 1 \\
\max \left\{a_{1}, a_{3}\right\}, & \text { if } \frac{l-1}{n} \leq e^{-1}<\frac{l}{n} \leq e^{-0.2}, w^{*}>1 \\
a_{2}, & \text { if } e^{-1}<\frac{l-1}{n}<\frac{l}{n} \leq e^{-0.2}, w^{*} \leq 1 \\
a_{4}, & \text { if } e^{-1}<\frac{l-1}{n}<\frac{l}{n} \leq e^{-0.2}, w^{*}>1 \\
\max \left\{a_{5}, a_{6}+\left(\ln \frac{l}{n}\right) \cdot a_{7}\right\}, & \text { if } e^{-1}<\frac{l-1}{n} \leq e^{-0.2}<\frac{l}{n}, w^{*} \leq 1, a_{7} \geq 0 \\
\max \left\{a_{5}, a_{6}+\ln \left(e^{-0.2}\right) \cdot a_{7}\right\}, & \text { if } e^{-1}<\frac{l-1}{n} \leq e^{-0.2}<\frac{l}{n}, w^{*} \leq 1, a_{7}<0 \\
\max \left\{a_{4}, a_{6}+\left(\ln \frac{l}{n}\right) \cdot a_{7}\right\}, & \text { if } e^{-1}<\frac{l-1}{n} \leq e^{-0.2}<\frac{l}{n}, w^{*}>1, a_{7} \geq 0 \\
\max \left\{a_{4}, a_{6}+\ln \left(e^{-0.2}\right) \cdot a_{7}\right\}, & \text { if } e^{-1}<\frac{l-1}{n} \leq e^{-0.2}<\frac{l}{n}, w^{*}>1, a_{7}<0 \\
a_{6}+\left(\ln \frac{l}{n}\right) \cdot a_{7}, & \text { if } e^{-0.2}<\frac{l-1}{n}, a_{7} \geq 0 \\
a_{6}+\left(\ln \frac{l-1}{n}\right) \cdot a_{7}, & \text { if } e^{-0.2}<\frac{l-1}{n}, a_{7}<0 .\end{cases}
\end{aligned}
$$

Then

$$
\begin{aligned}
\max _{t \in \bar{E}_{l}^{(n)}} \bar{h}_{l 3}^{(P N L, n)}(t)= & L_{\breve{4}}^{*}\left(\frac{l}{n}\right) \cdot w_{l 1}^{(* I, n)}+L_{1}^{*}\left(\frac{l}{n}\right) \cdot w_{l 3}^{(* I, n)}+R_{-1}^{*}\left(\frac{l-1}{n}\right) \cdot w_{l 2}^{(* I I, n)} \\
& +h_{3}\left[\mathfrak{w}_{l 3}^{(1, n)}, \mathfrak{w}_{l 3}^{(2, n)}, \mathfrak{w}_{l 3}^{(3, n)}, \mathfrak{w}_{l 3}^{(4, n)}, \mathfrak{w}_{l 3}^{(5, n)}, \mathfrak{w}_{l 3}^{(6, n)}, \mathfrak{w}_{l 3}^{(7, n)}, w_{l 1}^{(* I, n)}\right](t)
\end{aligned}
$$

- $\quad$ To find the maximum of $\bar{h}_{l 4}^{(P N U, n)}$ on $\bar{E}_{l}^{(n)}$, let

$$
\begin{aligned}
\mathfrak{w}_{l 4}^{1, n)} & =-0.8 w_{l 2}^{(* I, n)}-0.8 w_{l 3}^{(* I, n)}+0.8 w_{l 1}^{(* I I, n)}-2 \\
\mathfrak{w}_{l 4}^{(2, n)} & =-0.8 \cdot w_{l 2}^{(* I, n)}-0.8 w_{l 3}^{(* I, n)}+0.8 w_{l 1}^{(* I I, n)}-3 \\
\mathfrak{w}_{l 4}^{(3, n)} & =-w_{l 2}^{(* I, n)}-w_{l 3}^{(* I, n)}+w_{l 1}^{(* I I, n)}-3 \\
\mathfrak{w}_{l 4}^{(4, n)} & =-w_{l 2}^{(* I, n)}-w_{l 3}^{(* I, n)}+w_{l 1}^{(* I I, n)}-1 .
\end{aligned}
$$

Given any fixed real numbers $a_{1}, a_{2}, a_{3}, a_{4}$ that are regarded as the parameters, for sufficiently large $n$ satisfying $\frac{1}{n}<1-e^{-0.2}$, we define a parametric function

$$
h_{4}\left[a_{1}, a_{2}, a_{3}, a_{4}\right](t): \bar{E}_{l}^{(n)} \rightarrow \mathbb{R}
$$

by

$$
\begin{aligned}
& h_{4}\left[a_{1}, a_{2}, a_{3}, a_{4}\right](t) \\
& \quad= \begin{cases}a_{1}, & \text { if } \frac{l}{n} \leq e^{-1} \\
\max \left\{a_{1}, a_{2}-\ln \left(e^{-1}\right)\right\}, & \text { if } \frac{l-1}{n} \leq e^{-1}<\frac{l}{n} \leq e^{-0.2} \\
a_{2}-\ln \frac{l-1}{n}, & \text { if } e^{-1}<\frac{l-1}{n}<\frac{l}{n} \leq e^{-0.2} \\
\max \left\{a_{2}-\ln \frac{l-1}{n}, a_{3}+\left(\ln \frac{l}{n}\right) \cdot a_{4}\right\}, & \text { if } e^{-1}<\frac{l-1}{n} \leq e^{-0.2}<\frac{l}{n}, a_{4} \geq 0 \\
\max \left\{a_{2}-\ln \frac{l-1}{n}, a_{3}+\ln \left(e^{-0.2}\right) \cdot a_{4}\right\}, & \text { if } e^{-1}<\frac{l-1}{n} \leq e^{-0.2}<\frac{l}{n}, a_{4}<0 \\
a_{3}+\left(\ln \frac{l}{n}\right) \cdot a_{4}, & \text { if } e^{-0.2}<\frac{l-1}{n}, a_{4} \geq 0 \\
a_{3}+\left(\ln \frac{l-1}{n}\right) \cdot a_{4}, & \text { if } e^{-0.2}<\frac{l-1}{n}, a_{4}<0 .\end{cases}
\end{aligned}
$$


Then

$$
\begin{aligned}
\max _{t \in \bar{E}_{l}^{(n)}} \bar{h}_{l 4}^{(P N U, n)}(t)= & L_{\widetilde{1}}^{*}\left(\frac{l}{n}\right) \cdot w_{l 2}^{(* I, n)}+L_{\widetilde{1}}^{*}\left(\frac{l}{n}\right) \cdot w_{l 3}^{(* I, n)}+R_{-1}^{*}\left(\frac{l-1}{n}\right) \cdot w_{l 1}^{(* I I, n)} \\
& +h_{4}\left[\mathfrak{w}_{l 4}^{(1, n)}, \mathfrak{w}_{l 4}^{(2, n)}, \mathfrak{w}_{l 4}^{(3, n)}, \mathfrak{w}_{l 4}^{(4, n)}\right](t)
\end{aligned}
$$

- $\quad$ To find the maximum of $\bar{h}_{l 1}^{(N P U, n)}$ on $\bar{E}_{l}^{(n)}$, let

$$
\begin{aligned}
\mathfrak{w}_{l 1}^{(1, n)} & =-1.2 w_{l 1}^{(* I I, n)}-2.5 w_{l 2}^{(* I I, n)}-1.2 w_{l 3}^{(* I I, n)}+2.5 \\
\mathfrak{w}_{l 1}^{(2, n)} & =\ln \left(e^{-0.5}\right) \cdot\left(w_{l 2}^{(* I I, n)}-1\right)-1.2 w_{l 1}^{(* I I, n)}-2 w_{l 2}^{(* I I, n)}-1.2 w_{l 3}^{(* I I, n)}+2 \\
\mathfrak{w}_{l 1}^{(3, n)} & =\ln \frac{l}{n} \cdot\left(w_{l 2}^{(* I I, n)}-1\right)-1.2 w_{l 1}^{(* I I, n)}-2 w_{l 2}^{(* I I, n)}-1.2 w_{l 3}^{(* I I, n)}+2 \\
\mathfrak{w}_{l 1}^{(4, n)} & =\ln \frac{l-1}{n} \cdot\left(w_{l 2}^{(* I I, n)}-1\right)-1.2 w_{l 1}^{(* I I, n)}-2 w_{l 2}^{(* I I, n)}-1.2 w_{l 3}^{(* I I, n)}+2 \\
\mathfrak{w}_{l 1}^{(5, n)} & =\ln \left(e^{-0.2}\right) \cdot\left(w_{l 2}^{(* I I, n)}-1\right)-1.2 w_{l 1}^{(* I I, n)}-2 w_{l 2}^{(* I I, n)}-1.2 w_{l 3}^{(* I I, n)}+2 \\
\mathfrak{w}_{l 1}^{(6, n)} & =-w_{l 1}^{(* I I, n)}-2 w_{l 2}^{(* I I, n)}-w_{l 3}^{(* I I, n)}+2 \\
\mathfrak{w}_{l 1}^{(7, n)} & =w_{l 1}^{(* I I, n)}+w_{l 2}^{(* I I, n)}+w_{l 3}^{(* I I, n)}-1 .
\end{aligned}
$$

Given any fixed real numbers $a_{1}, a_{2}, a_{3}, a_{4}, a_{5}, a_{6}, a_{7}, w^{*}$ that are regarded as the parameters, for sufficiently large $n$ satisfying $\frac{1}{n}<1-e^{-0.2}$, we define a parametric function

$$
h_{5}\left[a_{1}, a_{2}, a_{3}, a_{4}, a_{5}, a_{6}, a_{7}, w^{*}\right](t): \bar{E}_{l}^{(n)} \rightarrow \mathbb{R}
$$

by

$$
\begin{aligned}
& h_{5}\left[a_{1}, a_{2}, a_{3}, a_{4}, a_{5}, a_{6}, a_{7}, w^{*}\right](t) \\
& \quad= \begin{cases}a_{1}, & \text { if } \frac{l}{n} \leq e^{-0.5} \\
\max \left\{a_{1}, a_{2}\right\}, & \text { if } \frac{l-1}{n} \leq e^{-0.5}<\frac{l}{n} \leq e^{-0.2}, w^{*} \leq 1 \\
\max \left\{a_{1}, a_{3}\right\}, & \text { if } \frac{l-1}{n} \leq e^{-0.5}<\frac{l}{n} \leq e^{-0.2}, w^{*}>1 \\
a_{4}, & \text { if } e^{-0.5}<\frac{l-1}{n}<\frac{l}{n} \leq e^{-0.2}, w^{*} \leq 1 \\
a_{3}, & \text { if } e^{-0.5}<\frac{l-1}{n}<\frac{l}{n} \leq e^{-0.2}, w^{*}>1 \\
\max \left\{a_{4}, a_{6}+\left(\ln \frac{l}{n}\right) \cdot a_{7}\right\}, & \text { if } e^{-0.5}<\frac{l-1}{n} \leq e^{-0.2}<\frac{l}{n}, w^{*} \leq 1, a_{7} \geq 0 \\
\max \left\{a_{4}, a_{6}+\ln \left(e^{-0.2}\right) \cdot a_{7}\right\}, & \text { if } e^{-0.5}<\frac{l-1}{n} \leq e^{-0.2}<\frac{l}{n}, w^{*} \leq 1, a_{7}<0 \\
\max \left\{a_{5}, a_{6}+\left(\ln \frac{l}{n}\right) \cdot a_{7}\right\}, & \text { if } e^{-0.5}<\frac{l-1}{n} \leq e^{-0.2}<\frac{l}{n}, w^{*}>1, a_{7} \geq 0 \\
\max \left\{a_{5}, a_{6}+\ln \left(e^{-0.2}\right) \cdot a_{7}\right\}, & \text { if } e^{-0.5}<\frac{l-1}{n} \leq e^{-0.2}<\frac{l}{n}, w^{*}>1, a_{7}<0 \\
a_{6}+\left(\ln \frac{l}{n}\right) \cdot a_{7}, & \text { if } e^{-0.2}<\frac{l-1}{n}, a_{7} \geq 0 \\
a_{6}+\left(\ln \frac{l-1}{n}\right) \cdot a_{7}, & \text { if } e^{-0.2}<\frac{l-1}{n}, a_{7}<0 .\end{cases}
\end{aligned}
$$

Then

$$
\begin{aligned}
\max _{t \in \bar{E}_{l}^{(n)}} \bar{h}_{l 1}^{(N P U, n)}(t)= & R_{\widetilde{1}}^{*}\left(\frac{l-1}{n}\right) \cdot w_{l 1}^{(* I I, n)}+R_{\widetilde{2}}^{*}\left(\frac{l-1}{n}\right) \cdot w_{l 2}^{(* I I, n)}+R_{\widetilde{1}}^{*}\left(\frac{l-1}{n}\right) \cdot w_{l 3}^{(* I I, n)} \\
& +h_{5}\left[\mathfrak{w}_{l 1}^{(1, n)}, \mathfrak{w}_{l 1}^{(2, n)}, \mathfrak{w}_{l 1}^{(3, n)}, \mathfrak{w}_{l 1}^{(4, n)}, \mathfrak{w}_{l 1}^{(5, n)}, \mathfrak{w}_{l 1}^{(6, n)}, \mathfrak{w}_{l 1}^{(7, n)}\right](t)
\end{aligned}
$$


- $\quad$ To find the maximum of $\bar{h}_{l 2}^{(N P U, n)}$ on $\bar{E}_{l}^{(n)}$, let

$$
\begin{aligned}
\mathfrak{w}_{l 2}^{(1, n)} & =-2.5 w_{l 1}^{(* I I, n)}-4 w_{l 2}^{(* I I, n)}+1.2 \\
\mathfrak{w}_{l 2}^{(2, n)} & =-2.5 w_{l 1}^{(* I I, n)}-3 w_{l 2}^{(* I I, n)}+1.2 \\
\mathfrak{w}_{l 2}^{(3, n)} & =\ln \frac{l}{n} \cdot\left(w_{l 1}^{(* I I, n)}+w_{l 2}^{(* I I, n)}\right)-2 w_{l 1}^{(* I I, n)}-3 w_{l 2}^{(* I I, n)}+1.2 \\
\mathfrak{w}_{l 2}^{(4, n)} & =\ln \left(e^{-0.5}\right) \cdot\left(w_{l 1}^{(* I I, n)}+w_{l 2}^{(* I I, n)}\right)-2 w_{l 1}^{(* I I, n)}-3 w_{l 2}^{(* I I, n)}+1.2 \\
\mathfrak{w}_{l 2}^{(5, n)} & =\ln \frac{l-1}{n} \cdot\left(w_{l 1}^{(* I I, n)}+w_{l 2}^{(* I I, n)}\right)-2 w_{l 1}^{(* I I, n)}-3 w_{l 2}^{(* I I, n)}+1.2 \\
\mathfrak{w}_{l 2}^{(6, n)} & =\ln \left(e^{-0.2}\right) \cdot\left(w_{l 1}^{(* I I, n)}+w_{l 2}^{(* I I, n)}\right)-2 w_{l 1}^{(* I I, n)}-3 w_{l 2}^{(* I I, n)}+1.2 \\
\mathfrak{w}_{l 2}^{(7, n)} & =-2 w_{l 1}^{(* I I, n)}-3 w_{l 2}^{(* I I, n)}+1 \\
\mathfrak{w}_{l 2}^{(8, n)} & =w_{l 1}^{(* I I, n)}+w_{l 2}^{(* I I, n)}-1 .
\end{aligned}
$$

Given any fixed real numbers $a_{1}, a_{2}, a_{3}, a_{4}, a_{5}, a_{6}, a_{7}, a_{8}, w_{1}^{*}, w_{2}^{*}$ that are regarded as the parameters, for sufficiently large $n$ satisfying $\frac{1}{n}<1-e^{-0.2}$, we define a parametric function

$$
h_{6}\left[a_{1}, a_{2}, a_{3}, a_{4}, a_{5}, a_{6}, a_{7}, a_{8}, w_{1}^{*}, w_{2}^{*}\right](t): \bar{E}_{l}^{(n)} \rightarrow \mathbb{R}
$$

by

$$
\begin{aligned}
& h_{6}\left[a_{1}, a_{2}, a_{3}, a_{4}, a_{5}, a_{6}, a_{7}, a_{8}, w_{1}^{*}, w_{2}^{*}\right](t) \\
& = \begin{cases}a_{1}, & \text { if } \frac{l}{n} \leq e^{-1} \\
\max \left\{a_{1}, a_{2}+\left(\ln \frac{l}{n}\right) w_{2}^{*}\right\}, & \text { if } \frac{l-1}{n} \leq e^{-1}<\frac{l}{n} \leq e^{-0.5}, w_{2}^{*} \geq 0 \\
\max \left\{a_{1}, a_{2}+\ln \left(e^{-1}\right) w_{2}^{*}\right\}, & \text { if } \frac{l-1}{n} \leq e^{-1}<\frac{l}{n} \leq e^{-0.5}, w_{2}^{*}<0 \\
a_{2}+\left(\ln \frac{l}{n}\right) w_{2}^{*}, & \text { if } e^{-1}<\frac{l-1}{n}<\frac{l}{n} \leq e^{-0.5}, w_{2}^{*} \geq 0 \\
a_{2}+\left(\ln \frac{l-1}{n}\right) w_{2}^{*}, & \text { if } e^{-1}<\frac{l-1}{n}<\frac{l}{n} \leq e^{-0.5}, w_{2}^{*}<0 \\
\max \left\{a_{2}+\ln \left(e^{-0.5}\right) w_{2}^{*}, a_{3}\right\}, & \text { if } \frac{l-1}{n} \leq e^{-0.5}<\frac{l}{n} \leq e^{-0.2}, w_{2}^{*} \geq 0, w_{1}^{*}+w_{2}^{*} \geq 0 \\
\max \left\{a_{2}+\ln \left(e^{-0.5}\right) w_{2}^{*}, a_{4}\right\}, & \text { if } \frac{l-1}{n} \leq e^{-0.5}<\frac{l}{n} \leq e^{-0.2}, w_{2}^{*} \geq 0, w_{1}^{*}+w_{2}^{*}<0 \\
\max \left\{a_{2}+\left(\ln \frac{l-1}{n}\right) w_{2}^{*}, a_{3}\right\}, & \text { if } \frac{l-1}{n} \leq e^{-0.5}<\frac{l}{n} \leq e^{-0.2}, w_{2}^{*}<0, w_{1}^{*}+w_{2}^{*} \geq 0 \\
\max \left\{a_{2}+\left(\ln \frac{l-1}{n}\right) w_{2}^{*}, a_{4}\right\}, & \text { if } \frac{l-1}{n} \leq e^{-0.5}<\frac{l}{n} \leq e^{-0.2}, w_{2}^{*}<0, w_{1}^{*}+w_{2}^{*}<0 \\
a_{3}, & \text { if } e^{-0.5}<\frac{l-1}{n}<\frac{l}{n} \leq e^{-0.2}, w_{1}^{*}+w_{2}^{*} \geq 0 \\
a_{5}, & \text { if } e^{-0.5}<\frac{l-1}{n}<\frac{l}{n} \leq e^{-0.2}, w_{1}^{*}+w_{2}^{*}<0 \\
\max \left\{a_{6}, a_{7}+\left(\ln \frac{l}{n}\right) a_{8}\right\}, & \text { if } e^{-0.5}<\frac{l-1}{n} \leq e^{-0.2}<\frac{l}{n}, w_{1}^{*}+w_{2}^{*} \geq 0, a_{8} \geq 0 \\
\max \left\{a_{6}, a_{7}+\ln \left(e^{-0.2}\right) a_{8}\right\}, & \text { if } e^{-0.5}<\frac{l-1}{n} \leq e^{-0.2}<\frac{l}{n}, w_{1}^{*}+w_{2}^{*} \geq 0, a_{8}<0 \\
\max \left\{a_{5}, a_{7}+\left(\ln \frac{l}{n}\right) a_{8}\right\}, & \text { if } e^{-0.5}<\frac{l-1}{n} \leq e^{-0.2}<\frac{l}{n}, w_{1}^{*}+w_{2}^{*}<0, a_{8} \geq 0 \\
\max \left\{a_{5}, a_{7}+\ln \left(e^{-0.2}\right) a_{8}\right\}, & \text { if } e^{-0.5}<\frac{l-1}{n} \leq e^{-0.2}<\frac{l}{n}, w_{1}^{*}+w_{2}^{*}<0, a_{8}<0 \\
a_{7}+\left(\ln \frac{l}{n}\right) a_{8}, & \text { if } e^{-0.2}<\frac{l-1}{n}, a_{8} \geq 0 \\
a_{7}+\left(\ln \frac{l-1}{n}\right) a_{8}, & \text { if } e^{-0.2}<\frac{l-1}{n}, a_{8}<0 .\end{cases}
\end{aligned}
$$

Then

$$
\begin{aligned}
& \max _{t \in \bar{E}_{l}^{(n)}} \bar{h}_{l 2}^{(N P U, n)}(t)=R_{\widetilde{2}}^{*}\left(\frac{l-1}{n}\right) \cdot w_{l 1}^{(* I I, n)}+R_{\widetilde{3}}^{*}\left(\frac{l-1}{n}\right) \cdot w_{l 2}^{(* I I, n)} \\
& \quad+h_{6}\left[\mathfrak{w}_{l 2}^{(1, n)}, \mathfrak{w}_{l 2}^{(2, n)}, \mathfrak{w}_{l 2}^{(3, n)}, \mathfrak{w}_{l 2}^{(4, n)}, \mathfrak{w}_{l 2}^{(5, n)}, \mathfrak{w}_{l 2}^{(6, n)}, \mathfrak{w}_{l 2}^{(7, n)}, \mathfrak{w}_{l 2}^{(8, n)}, w_{l 1}^{(* I I, n)}, w_{l 2}^{(* I I, n)}\right](t)
\end{aligned}
$$


- $\quad$ To find the maximum of $\bar{h}_{l 3}^{(N P U, n)}$ on $\bar{E}_{l}^{(n)}$, let

$$
\begin{aligned}
& \mathfrak{w}_{l 3}^{(1, n)}=-5 w_{l 1}^{(* I I, n)}-1.2 w_{l 3}^{(* I I, n)}+1.2 w_{l 2}^{(* I, n)}+6 \\
& \mathfrak{w}_{l 3}^{(2, n)}=\ln \left(e^{-1}\right) \cdot\left(w_{l 1}^{(* I I, n)}-1\right)-4 w_{l 1}^{(* I I, n)}-1.2 w_{l 3}^{(* I I, n)}+1.2 w_{l 2}^{(* I, n)}+5 \\
& \mathfrak{w}_{l 3}^{(3, n)}=\ln \frac{l}{n} \cdot\left(w_{l 1}^{(* I I, n)}-1\right)-4 w_{l 1}^{(* I I, n)}-1.2 w_{l 3}^{(* I I, n)}+1.2 w_{l 2}^{(* I, n)}+5 \\
& \mathfrak{w}_{l 3}^{(4, n)}=\ln \frac{l-1}{n} \cdot\left(w_{l 1}^{(* I I, n)}-1\right)-4 w_{l 1}^{(* I I, n)}-1.2 w_{l 3}^{(* I I, n)}+1.2 w_{l 2}^{(* I, n)}+5 \\
& \mathfrak{w}_{l 3}^{(5, n)}=\ln \left(e^{-0.2}\right) \cdot\left(w_{l 1}^{(* I I, n)}-1\right)-4 w_{l 1}^{(* I I, n)}-1.2 w_{l 3}^{(* I I, n)}+1.2 w_{l 2}^{(* I, n)}+5 \\
& \mathfrak{w}_{l 3}^{(6, n)}=-4 w_{l 1}^{(* I I, n)}-w_{l 3}^{(* I I, n)}+w_{l 2}^{(* I, n)}+5 \\
& \mathfrak{w}_{l 3}^{(7, n)}=w_{l 1}^{(* I I, n)}+w_{l 3}^{(* I I, n)}-w_{l 2}^{(* I, n)}-1 .
\end{aligned}
$$

Given any fixed real numbers $a_{1}, a_{2}, a_{3}, a_{4}, a_{5}, a_{6}, a_{7}, w^{*}$ that are regarded as the parameters, for sufficiently large $n$ satisfying $\frac{1}{n}<1-e^{-0.2}$, we define a parametric function

$$
h_{7}\left[a_{1}, a_{2}, a_{3}, a_{4}, a_{5}, a_{6}, a_{7}, w^{*}\right](t): \bar{E}_{l}^{(n)} \rightarrow \mathbb{R}
$$

by

$$
\begin{aligned}
& h_{7}\left[a_{1}, a_{2}, a_{3}, a_{4}, a_{5}, a_{6}, a_{7}, w^{*}\right](t) \\
& \quad= \begin{cases}a_{1}, & \text { if } \frac{l}{n} \leq e^{-1} \\
\max \left\{a_{1}, a_{2}\right\}, & \text { if } \frac{l-1}{n} \leq e^{-1}<\frac{l}{n} \leq e^{-0.2}, w^{*} \leq 1 \\
\max \left\{a_{1}, a_{3}\right\}, & \text { if } \frac{l-1}{n} \leq e^{-1}<\frac{l}{n} \leq e^{-0.2}, w^{*}>1 \\
a_{4}, & \text { if } e^{-1}<\frac{l-1}{n}<\frac{l}{n} \leq e^{-0.2}, w^{*} \leq 1 \\
a_{3}, & \text { if } e^{-1}<\frac{l-1}{n}<\frac{l}{n} \leq e^{-0.2}, w^{*}>1 \\
\max \left\{a_{4}, a_{6}+\left(\ln \frac{l}{n}\right) \cdot a_{7}\right\}, & \text { if } e^{-1}<\frac{l-1}{n} \leq e^{-0.2}<\frac{l}{n}, w^{*} \leq 1, a_{7} \geq 0 \\
\max \left\{a_{4}, a_{6}+\ln \left(e^{-0.2}\right) \cdot a_{7}\right\}, & \text { if } e^{-1}<\frac{l-1}{n} \leq e^{-0.2}<\frac{l}{n}, w^{*} \leq 1, a_{7}<0 \\
\max \left\{a_{5}, a_{6}+\left(\ln \frac{l}{n}\right) \cdot a_{7}\right\}, & \text { if } e^{-1}<\frac{l-1}{n} \leq e^{-0.2}<\frac{l}{n}, w^{*}>1, a_{7} \geq 0 \\
\max \left\{a_{5}, a_{6}+\ln \left(e^{-0.2}\right) \cdot a_{7}\right\}, & \text { if } e^{-1}<\frac{l-1}{n} \leq e^{-0.2}<\frac{l}{n}, w^{*}>1, a_{7}<0 \\
a_{6}+\left(\ln \frac{l}{n}\right) \cdot a_{7}, & \text { if } e^{-0.2}<\frac{l-1}{n}, a_{7} \geq 0 \\
a_{6}+\left(\ln \frac{l-1}{n}\right) \cdot a_{7}, & \text { if } e^{-0.2}<\frac{l-1}{n}, a_{7}<0 .\end{cases}
\end{aligned}
$$

Then

$$
\begin{aligned}
\max _{t \in \bar{E}_{l}^{(n)}} \bar{h}_{l 3}^{(N P U, n)}(t)= & R_{\widetilde{4}}^{*}\left(\frac{l-1}{n}\right) \cdot w_{l 1}^{(* I I, n)}+R_{\widetilde{1}}^{*}\left(\frac{l-1}{n}\right) \cdot w_{l 3}^{(* I I, n)}+L_{\widetilde{-1}}^{*}\left(\frac{l}{n}\right) \cdot w_{l 2}^{(* I, n)} \\
& +h_{7}\left[\mathfrak{w}_{l 3}^{(1, n)}, \mathfrak{w}_{l 3}^{(2, n)}, \mathfrak{w}_{l 3}^{(3, n)}, \mathfrak{w}_{l 3}^{(4, n)}, \mathfrak{w}_{l 3}^{(5, n)}, \mathfrak{w}_{l 3}^{(6, n)}, \mathfrak{w}_{l 3}^{(7, n)}, w_{l 1}^{(* I I, n)}\right](t)
\end{aligned}
$$

- To find the maximum of $\bar{h}_{l 4}^{(N P L, n)}$ on $\bar{E}_{l}^{(n)}$, let

$$
\begin{aligned}
& \mathfrak{w}_{l 4}^{(1, n)}=-1.2 w_{l 2}^{(* I I, n)}-1.2 w_{l 3}^{(* I I, n)}+1.2 w_{l 1}^{(* I, n)}-4 \\
& \mathfrak{w}_{l 4}^{(2, n)}=-1.2 w_{l 2}^{(* I I, n)}-1.2 w_{l 3}^{(* I I, n)}+1.2 w_{l 1}^{(* I, n)}-3 \\
& \mathfrak{w}_{l 4}^{(3, n)}=-w_{l 2}^{(* I I, n)}-w_{l 3}^{(* I I, n)}+w_{l 1}^{(* I, n)}-3 \\
& \mathfrak{w}_{l 4}^{(4, n)}=w_{l 2}^{(* I I, n)}+w_{l 3}^{(* I I, n)}-w_{l 1}^{(* I, n)}+1 .
\end{aligned}
$$


Given any fixed real numbers $a_{1}, a_{2}, a_{3}, a_{4}$ that are regarded as the parameters, for sufficiently large $n$ satisfying $\frac{1}{n}<1-e^{-0.2}$, we define a parametric function

$$
h_{8}\left[a_{1}, a_{2}, a_{3}, a_{4}\right](t): \bar{E}_{l}^{(n)} \rightarrow \mathbb{R}
$$

by

$$
\begin{aligned}
& h_{8}\left[a_{1}, a_{2}, a_{3}, a_{4}\right](t) \\
& \quad= \begin{cases}a_{1}, & \text { if } \frac{l}{n} \leq e^{-1} \\
\max \left\{a_{1}, a_{2}+\ln \frac{l}{n}\right\}, & \text { if } \frac{l-1}{n} \leq e^{-1}<\frac{l}{n} \leq e^{-0.2} \\
a_{2}+\ln \frac{l}{n}, & \text { if } e^{-1}<\frac{l-1}{n}<\frac{l}{n} \leq e^{-0.2} \\
\max \left\{a_{2}+\ln \left(e^{-0.2}\right), a_{3}+\left(\ln \frac{l}{n}\right) \cdot a_{4}\right\}, & \text { if } e^{-1}<\frac{l-1}{n} \leq e^{-0.2}<\frac{l}{n}, a_{4} \geq 0 \\
\max \left\{a_{2}+\ln \left(e^{-0.2}\right), a_{3}+\ln \left(e^{-0.2}\right) \cdot a_{4}\right\}, & \text { if } e^{-1}<\frac{l-1}{n} \leq e^{-0.2}<\frac{l}{n}, a_{4}<0 \\
a_{3}+\left(\ln \frac{l}{n}\right) \cdot a_{4}, & \text { if } e^{-0.2}<\frac{l-1}{n}, a_{4} \geq 0 \\
a_{3}+\left(\ln \frac{l-1}{n}\right) \cdot a_{4}, & \text { if } e^{-0.2}<\frac{l-1}{n}, a_{4}<0 .\end{cases}
\end{aligned}
$$

Then

$$
\begin{aligned}
\max _{t \in \bar{E}_{l}^{(n)}} \bar{h}_{l 4}^{(N P L, n)}(t)= & R_{\widetilde{1}}^{*}\left(\frac{l-1}{n}\right) \cdot w_{l 2}^{(* I I, n)}+R_{\widetilde{1}}^{*}\left(\frac{l-1}{n}\right) \cdot w_{l 3}^{(* I I, n)}+L_{-1}^{*}\left(\frac{l}{n}\right) \cdot w_{l 1}^{(* I, n)} \\
& +h_{8}\left[\mathfrak{w}_{l 4}^{(1, n)}, \mathfrak{w}_{l 4}^{(2, n)}, \mathfrak{w}_{l 4}^{(3, n)}, \mathfrak{w}_{l 4}^{(4, n)}\right](t)
\end{aligned}
$$

From (243)-(246), we obtain

$$
\begin{aligned}
& \pi_{l}^{(P N L, n)=\max }\left\{-L_{\frac{2}{*}}^{*}\left(\frac{l-1}{n}\right)+\max _{t \in \bar{E}_{l}^{(n)}} \bar{h}_{l 1}^{(P N L, n)}(t),-L_{\tilde{1}}^{*}\left(\frac{l-1}{n}\right)+\max _{t \in \bar{E}_{l}^{(n)}} \bar{h}_{l 2}^{(P N L, n)}(t),\right. \\
& \left.-\quad L_{\tilde{5}}^{*}\left(\frac{l-1}{n}\right)+\max _{t \in \bar{E}_{l}^{(n)}} \bar{h}_{l 3}^{(P N L, n)}(t)\right\} \\
& \pi_{l}^{(P N U, n)=-R_{-3}^{*}}\left(\frac{l}{n}\right)+\max _{t \in \bar{E}_{l}^{(n)}} \bar{h}_{l 4}^{(P N U, n)}(t) \\
& \pi_{l}^{(N P L, n)=-L_{-3}^{*}}\left(\frac{l-1}{n}\right)+\max _{t \in \bar{E}_{l}^{(n)}} \bar{h}_{l 4}^{(N P L, n)}(t) \\
& \pi_{l}^{(N P U, n)=\max }\left\{-R_{\widetilde{2}}^{*}\left(\frac{l}{n}\right)+\max _{t \in \bar{E}_{l}^{(n)}} \bar{h}_{l 1}^{(N P U, n)}(t),-R_{\widetilde{1}}^{*}\left(\frac{l}{n}\right)+\max _{t \in \bar{E}_{l}^{(n)}} \bar{h}_{l 2}^{(N P U, n)}(t)\right. \\
& \left.-R_{\tilde{5}}^{*}\left(\frac{l}{n}\right)+\max _{t \in \bar{E}_{l}^{(n)}} \bar{h}_{l 3}^{(N P U, n)}(t)\right\} .
\end{aligned}
$$

Then

$$
\pi_{l}^{(P N, n)}=\max \left\{\pi_{l}^{(P N L, n)}, \pi_{l}^{(P N U, n)}\right\} \text { and } \pi_{l}^{(N P, n)}=\max \left\{\pi_{l}^{(N P L, n)}, \pi_{l}^{(N P U, n)}\right\} .
$$


We can calculate the error estimation $\varepsilon_{n}$ according to (247) in which the following integrals should be evaluated using (248) and (249). Let $\mathfrak{c}_{i}^{L}=e^{\breve{c}_{i}^{L}-\breve{c}_{i}}$ and $\mathfrak{c}_{i}^{U}=e^{\breve{c}_{i}-\breve{c}_{i}^{U}}$. Then

$$
\begin{gathered}
\int_{\frac{l-1}{n}}^{\frac{l}{n}} L_{\widetilde{c}_{i}}^{*}(t) d t= \begin{cases}\int_{\frac{l-1}{n}}^{\frac{l}{n}} \breve{c}_{i}^{L} d t, & \text { if } 0 \leq \frac{l}{n} \leq \mathfrak{c}_{i}^{L} \\
\int_{\frac{l-1}{n}}^{\mathfrak{c}_{i}^{L}} \breve{c}_{i}^{L} d t+\int_{\mathfrak{c}_{i}^{L}}^{\frac{l}{n}}\left(\breve{c}_{i}+\ln t\right) d t, & \text { if } \frac{l-1}{n}<\mathfrak{c}_{i}^{L}<\frac{l}{n} \\
\int_{\frac{l-1}{n}}^{\frac{l}{n}}\left(\breve{c}_{i}+\ln t\right) d t, & \text { if } \mathfrak{c}_{i}^{L} \leq \frac{l-1}{n} \leq 1\end{cases} \\
= \begin{cases}\frac{1}{n} \cdot \breve{c}_{i}^{L}, & \text { if } 0 \leq \frac{l}{n} \leq \mathfrak{c}_{i}^{L} \\
\breve{c}_{i} \cdot \frac{l}{n}-\breve{c}_{i}^{L} \cdot \frac{l-1}{n}+\mathfrak{c}_{i}^{L} \cdot\left(1-\ln \mathfrak{c}_{i}^{L}+\breve{c}_{i}^{L}-\breve{c}_{i}\right)+\frac{l}{n} \cdot\left(\ln \frac{l}{n}-1\right), & \text { if } \frac{l-1}{n}<\mathfrak{c}_{i}^{L}<\frac{l}{n} \\
\frac{1}{n} \cdot\left[\breve{c}_{i}+(l-1) \cdot \ln \frac{l}{l-1}+\ln \frac{l}{n}-1\right], & \text { if } \mathfrak{c}_{i}^{L} \leq \frac{l-1}{n} \leq 1\end{cases}
\end{gathered}
$$

and

$$
\begin{gathered}
\int_{\frac{l-1}{n}}^{\frac{l}{n}} R_{\widetilde{c}_{i}}^{*}(t) d t= \begin{cases}\int_{\frac{l-1}{n}}^{\frac{l}{n}} \breve{c}_{i}^{U} d t, & \text { if } 0 \leq \frac{l}{n} \leq \mathfrak{c}_{i}^{U} \\
\int_{\frac{l-1}{n}}^{\mathfrak{c}_{i}^{U}} \breve{c}_{i}^{U} d t+\int_{\mathfrak{c}_{i}^{U}}^{\frac{l}{n}}\left(\breve{c}_{i}-\ln t\right) d t, & \text { if } \frac{l-1}{n}<\mathfrak{c}_{i}^{U}<\frac{l}{n} \\
\int_{\frac{l-1}{n}}^{\frac{l}{n}}\left(\breve{c}_{i}-\ln t\right) d t, & \text { if } \mathfrak{c}_{i}^{U} \leq \frac{l-1}{n} \leq 1\end{cases} \\
= \begin{cases}\frac{1}{n} \cdot \breve{c}_{i}^{U}, & \text { if } 0 \leq \frac{l}{n} \leq \mathfrak{c}_{i}^{U} \\
\breve{c}_{i} \cdot \frac{l}{n}-\breve{c}_{i}^{U} \cdot \frac{l-1}{n}+\mathfrak{c}_{i}^{U} \cdot\left(\ln \mathfrak{c}_{i}^{U}-1+\breve{c}_{i}^{U}-\breve{c}_{i}\right)+\frac{l}{n} \cdot\left(1-\ln \frac{l}{n}\right), & \text { if } \frac{l-1}{n}<\mathfrak{c}_{i}^{U}<\frac{l}{n} \\
\frac{1}{n} \cdot\left[\breve{c}_{i}-(l-1) \cdot \ln \frac{l}{l-1}-\ln \frac{l}{n}+1\right], & \text { if } \mathfrak{c}_{i}^{U} \leq \frac{l-1}{n} \leq 1\end{cases}
\end{gathered}
$$

We denote by

$$
\begin{aligned}
V\left(S O P_{n}\right) & =\sum_{j=1}^{q} \int_{0}^{1} a_{j}^{L}(t) \cdot x_{j}^{(L, n)}(t) d t+\sum_{j=1}^{q} \int_{0}^{1} a_{j}^{U}(t) \cdot x_{j}^{(U, n)}(t) d t \\
& =\sum_{j=1}^{q} \sum_{l=1}^{n} z_{l j}^{(L, n)} \cdot \int_{\frac{l-1}{n}}^{\frac{l}{n}} L_{\widetilde{a}_{j}}^{*}(t) d t+\sum_{j=1}^{q} \sum_{l=1}^{n} z_{l j}^{(U, n)} \cdot \int_{\frac{l-1}{n}}^{\frac{l}{n}} R_{\widetilde{a}_{j}}^{*}(t) d t(u \operatorname{sing}(233))
\end{aligned}
$$

the approximate optimal objective value of problem SOP. Theorems 3 and 4 say that

$$
0 \leq V(S O P)-V\left(S O P_{n}\right) \leq \varepsilon_{n}
$$

and

$$
0 \leq V\left(S O P_{n}\right)-V\left(P_{n}\right) \leq V(S O P)-V\left(P_{n}\right) \leq \varepsilon_{n}
$$

In the following Table 1, we present the error estimation $\varepsilon_{n}$ for the different values of $n$. We also calculate the relative error of $\varepsilon_{n}$ with respect to $V\left(P_{n}\right)$ given by $\varepsilon_{n}^{*}=\varepsilon_{n} / V\left(P_{n}\right)$. 
Table 1. Numerical results.

\begin{tabular}{ccccc}
\hline$n$ & $\varepsilon_{n}$ & $V\left(P_{n}\right)$ & $V\left(S O P_{n}\right)$ & $\varepsilon_{n}^{*}$ \\
\hline 10 & 12.8321 & 18.0617 & 18.2663 & 0.7104603 \\
100 & 1.3985 & 18.5838 & 18.6047 & 0.0752535 \\
500 & 0.2632 & 18.6290 & 18.6332 & 0.0126818 \\
1000 & 0.1394 & 18.6351 & 18.6372 & 0.0074799 \\
1500 & 0.0892 & 18.6362 & 18.6376 & 0.0047858 \\
2000 & 0.0653 & 18.6377 & 18.6387 & 0.0030207 \\
3000 & 0.0427 & 18.6385 & 18.6392 & 0.0022929 \\
\hline
\end{tabular}

Suppose that the decision-maker can tolerate the relative error $\varepsilon_{n}^{*}=0.005$. Then we see that $n=1500$ is sufficient to achieve this relative error $\varepsilon_{n}^{*}$.

The $\varepsilon_{n}$-optimal solution of problem SOP can be obtained according to Theorem 5 , and the $\varepsilon_{n}$-nondominated optimal solution of fuzzy linear programming problem FOP can be obtained according to Theorem 1 . The figures of the $\varepsilon_{n}$-optimal solutions for $n=50$ and $n=100$ are shown in appendix where the membership functions of $\tilde{x}_{2}$ and $\tilde{x}_{4}$ are zero functions. We also remark that the left parts of the membership functions of $\tilde{x}_{1}$ and $\tilde{x}_{3}$ are $x_{1}^{(L, n)}$ and $x_{3}^{(L, n)}$, respectively, and the right parts of the membership functions of $\tilde{x}_{1}$ and $\tilde{x}_{3}$ are $x_{1}^{(U, n)}$ and $x_{3}^{(U, n)}$, respectively.

Suppose that each quantity in the original fuzzy linear programming problem is assumed to be a real number; that is, we consider the following linear programming problem

$$
\begin{array}{ll}
\max & 2 x_{1}+x_{2}+5 x_{3}-3 x_{4} \\
\text { subject to } & x_{1}+2 x_{2}+4 x_{3}-x_{4} \leq 6 \\
& 2 x_{1}+3 x_{2}-x_{3}+x_{4} \leq 12 \\
& x_{1}+x_{3}+x_{4} \leq 4 \\
& x_{1}, x_{2}, x_{3}, x_{4} \in \mathbb{R}_{+} .
\end{array}
$$

Then the optimal solution is $\left(x_{1}, x_{2}, x_{3}, x_{4}\right)=(10 / 3,0,2 / 3,0)$ that is compatible with the fuzzy solution presented in the Appendix A.

\subsection{Using the Triangular Fuzzy Numbers}

We consider the triangular fuzzy number $\widetilde{a}=\left(\breve{a}^{L}, \breve{a}, \breve{a}^{U}\right)$ with the membership functions defined by

$$
\xi_{\widetilde{a}}(r)= \begin{cases}\frac{r-\breve{a}^{L}}{\breve{a}-\breve{a}^{L}} & \text { if } \breve{a}^{L} \leq r \leq \breve{a} \\ \frac{\breve{a}^{U}-r}{\breve{a}^{U}-\breve{a}} & \text { if } \breve{a}<r \leq \breve{a}^{U} \\ 0 & \text { otherwise. }\end{cases}
$$

Then the $\alpha$-level set is given by

$$
\widetilde{a}_{\alpha}=\left[(1-\alpha) \cdot \breve{a}^{L}+\alpha \cdot \breve{a},(1-\alpha) \cdot \breve{a}^{U}+\alpha \cdot \breve{a}\right] ;
$$

that is,

$$
\widetilde{a}_{\alpha}^{L}=(1-\alpha) \cdot \breve{a}^{L}+\alpha \cdot \breve{a} \text { and } \widetilde{a}_{\alpha}^{U}=(1-\alpha) \cdot \breve{a}^{U}+\alpha \cdot \breve{a} .
$$

Assume that all of the fuzzy numbers in the fuzzy linear programming problem FLP are taken to be the triangular fuzzy numbers given by

$$
\widetilde{a}_{j}=\left(\breve{a}_{j}^{L}, \breve{a}_{j}, \breve{a}_{j}^{U}\right), \quad \widetilde{c}_{i}=\left(\breve{c}_{i}^{L}, \breve{c}_{i}, \breve{c}_{i}^{U}\right) \text { and } \widetilde{b}_{i j}=\left(\breve{b}_{i j}^{L}, \breve{b}_{i j}, \breve{b}_{i j}^{U}\right)
$$


for $i=1, \cdots, n$ and $j=1, \cdots, q$. Then, according to the previous notations, we have the following real-valued functions defined on $[0,1]$ :

$$
\begin{aligned}
& a_{j}^{L}(t)=(1-t) \cdot \breve{a}_{j}^{L}+t \cdot \breve{a}_{j} \text { and } a_{j}^{U}(t)=(1-t) \cdot \breve{a}_{j}^{U}+t \cdot \breve{a}_{j} ; \\
& c_{i}^{L}(t)=(1-t) \cdot \breve{c}_{i}^{L}+t \cdot \breve{c}_{i} \text { and } c_{i}^{U}(t)=(1-t) \cdot \breve{c}_{i}^{U}+t \cdot \breve{c}_{i} ; \\
& b_{i j}^{L}(t)=(1-t) \cdot \breve{b}_{i j}^{L}+t \cdot \breve{b}_{i j} \text { and } b_{i j}^{U}(t)=(1-t) \cdot \breve{b}_{i j}^{U}+t \cdot \breve{b}_{i j} .
\end{aligned}
$$

For $l=1, \cdots, n$, from (81) and (82), we see that

$$
\begin{aligned}
\mathfrak{b}_{l}^{(P N, n)}=\frac{1}{n} \cdot \min _{j=1, \cdots, q}\left\{(n-l+1) \cdot \sum_{\left\{i: j \in P_{i}\right\}} \breve{b}_{i j}^{L}+(l-1) \cdot \sum_{\left\{i: j \in P_{i}\right\}} \breve{b}_{i j}\right. \\
\left.+(n-l) \cdot \sum_{\left\{i: j \in N_{i}\right\}} \breve{b}_{i j}^{U}+l \cdot \sum_{\left\{i: j \in N_{i}\right\}} \breve{b}_{i j}\right\}
\end{aligned}
$$

and

$$
\begin{aligned}
\mathfrak{b}_{l}^{(N P, n)}=\frac{1}{n} \cdot \min _{j=1, \cdots, q}\left\{(n-l+1) \cdot \sum_{\left\{i: j \in N_{i}\right\}} \breve{b}_{i j}^{L}+(l-1) \cdot \sum_{\left\{i: j \in N_{i}\right\}} \breve{b}_{i j}\right. \\
\left.+(n-l) \cdot \sum_{\left\{i: j \in P_{i}\right\}} \breve{b}_{i j}^{U}+l \cdot \sum_{\left\{i: j \in P_{i}\right\}} \breve{b}_{i j}\right\} .
\end{aligned}
$$

From (42)-(44), we have

$$
\begin{aligned}
& a_{l j}^{(L, n)}=a_{j}^{L}\left(\frac{l-1}{n}\right)=\frac{1}{n} \cdot\left[(n-l+1) \cdot \breve{a}_{j}^{L}+(l-1) \cdot \breve{a}_{j}\right] \\
& a_{l j}^{(U, n)}=a_{j}^{U}\left(\frac{l}{n}\right)=\frac{1}{n} \cdot\left[(n-l) \cdot \breve{a}_{j}^{U}+l \cdot \breve{a}_{j}\right] \\
& c_{l i}^{(L, n)}=c_{i}^{L}\left(\frac{l-1}{n}\right)=\frac{1}{n} \cdot\left[(n-l+1) \cdot \breve{c}_{i}^{L}+(l-1) \cdot \breve{c}_{i}\right] \\
& c_{l i}^{(U, n)}=c_{i}^{U}\left(\frac{l}{n}\right)=\frac{1}{n} \cdot\left[(n-l) \cdot \breve{c}_{i}^{U}+l \cdot \breve{c}_{i}\right] \\
& b_{l i j}^{(L, n)}=b_{i j}^{L}\left(\frac{l}{n}\right)=\frac{1}{n} \cdot\left[(n-l) \cdot \breve{b}_{i j}^{L}+l \cdot \breve{b}_{i j}\right] \\
& b_{l i j}^{(U, n)}=b_{i j}^{U}\left(\frac{l-1}{n}\right)=\frac{1}{n} \cdot\left[(n-l+1) \cdot \breve{b}_{i j}^{U}+(l-1) \cdot \breve{b}_{i j}\right] .
\end{aligned}
$$

In order to evaluate $\pi_{l}^{(P N, n)}$ and $\pi_{l}^{(N P, n)}$ presented in (76), we need to solve (214)-(217). From (213), we need to calculate

$$
\begin{aligned}
& \widehat{h}_{l j}^{(P N, n)}=\sum_{\left\{i: j \in P_{i}\right\}} b_{l i j}^{(L, n)} \cdot w_{l i}^{(* I, n)}+\sum_{\left\{i: j \in N_{i}\right\}} b_{l i j}^{(U, n)} \cdot w_{l i}^{(* I I, n)} \\
& =\frac{1}{n} \cdot \sum_{\left\{i: j \in P_{i}\right\}}\left[(n-l) \cdot \breve{b}_{i j}^{L}+l \cdot \breve{b}_{i j}\right] \cdot w_{l i}^{(* I, n)}+\frac{1}{n} \cdot \sum_{\left\{i: j \in N_{i}\right\}}\left[(n-l+1) \cdot \breve{b}_{i j}^{U}+(l-1) \cdot \breve{b}_{i j}\right] \cdot w_{l i}^{(* I, n)}
\end{aligned}
$$

(using (255) and (256)) 
and

$$
\begin{aligned}
& \widehat{h}_{l j}^{(N P, n)}=\sum_{\left\{i: j \in N_{i}\right\}} b_{l i j}^{(L, n)} \cdot w_{l i}^{(* I, n)}+\sum_{\left\{i: j \in P_{i}\right\}} b_{l i j}^{(U, n)} \cdot w_{l i}^{(* I I, n)} \\
& \quad=\frac{1}{n} \cdot \sum_{\left\{i: j \in N_{i}\right\}}\left[(n-l) \cdot \breve{b}_{i j}^{L}+l \cdot \breve{b}_{i j}\right] \cdot w_{l i}^{(* I, n)}+\frac{1}{n} \cdot \sum_{\left\{i: j \in P_{i}\right\}}\left[(n-l+1) \cdot \breve{b}_{i j}^{U}+(l-1) \cdot \breve{b}_{i j}\right] \cdot w_{l i}^{(* I I, n)} .
\end{aligned}
$$

and

$$
\begin{aligned}
\breve{h}_{l j}^{(P N, n)}(t)=-\sum_{\left\{i: j \in P_{i}\right\}} b_{i j}^{L}(t) \cdot w_{l i}^{(* I, n)}-\sum_{\left\{i: j \in N_{i}\right\}} b_{i j}^{U}(t) \cdot w_{l i}^{(* I I, n)} \\
=-\sum_{\left\{i: j \in P_{i}\right\}}\left[(1-t) \cdot \breve{b}_{i j}^{L}+t \cdot \breve{b}_{i j}\right] \cdot w_{l i}^{(* I, n)}-\sum_{\left\{i: j \in N_{i}\right\}}\left[(1-t) \cdot \breve{b}_{i j}^{U}+t \cdot \breve{b}_{i j}\right] \cdot w_{l i}^{(* I I, n)} \text { (using (252)) } \\
=-\sum_{\left\{i: j \in P_{i}\right\}} \breve{b}_{i j}^{L} \cdot w_{l i}^{(* I, n)}-\sum_{\left\{i: j \in N_{i}\right\}} \breve{b}_{i j}^{U} \cdot w_{l i}^{(* I I, n)} \\
+t \cdot\left[\sum_{\left\{i: j \in P_{i}\right\}}\left(\breve{b}_{i j}^{L}-\breve{b}_{i j}\right) \cdot w_{l i}^{(* I, n)}+\sum_{\left\{i: j \in N_{i}\right\}}\left(\breve{b}_{i j}^{U}-\breve{b}_{i j}\right) \cdot w_{l i}^{(* I I, n)}\right]
\end{aligned}
$$

and

$$
\begin{aligned}
\breve{h}_{l j}^{(N P, n)}(t)=-\sum_{\left\{i: j \in N_{i}\right\}} b_{i j}^{L}(t) \cdot w_{l i}^{(* I, n)}-\sum_{\left\{i: j \in P_{i}\right\}} b_{i j}^{U}(t) \cdot w_{l i}^{(* I I, n)} \\
=-\sum_{\left\{i: j \in N_{i}\right\}} \breve{b}_{i j}^{L} \cdot w_{l i}^{(* I, n)}-\sum_{\left\{i: j \in P_{i}\right\}} \breve{b}_{i j}^{U} \cdot w_{l i}^{(* I I, n)} \\
+t \cdot\left[\sum_{\left\{i: j \in N_{i}\right\}}\left(\breve{b}_{i j}^{L}-\breve{b}_{i j}\right) \cdot w_{l i}^{(* I, n)}+\sum_{\left\{i: j \in P_{i}\right\}}\left(\breve{b}_{i j}^{U}-\breve{b}_{i j}\right) \cdot w_{l i}^{(* I I, n)}\right]
\end{aligned}
$$

Using (250), (213), (218) and (219), we also have

$$
\begin{aligned}
& \bar{h}_{l j}^{(P N L, n)}(t)=h_{l j}^{(P N, n)}(t)+a_{j}^{L}(t)=\widehat{h}_{l j}^{(P N, n)}+\breve{h}_{l j}^{(P N, n)}(t)+\breve{a}_{j}^{L}+t \cdot\left(\breve{a}_{j}-\breve{a}_{j}^{L}\right) \text { for } j \in P \\
& \bar{h}_{l j}^{(N P U, n)}(t)=h_{l j}^{(N P, n)}(t)+a_{j}^{U}(t)=\widehat{h}_{l j}^{(N P, n)}+\breve{h}_{l j}^{(N P, n)}(t)+\breve{a}_{j}^{U}+t \cdot\left(\breve{a}_{j}-\breve{a}_{j}^{U}\right) \text { for } j \in P \\
& \bar{h}_{l j}^{(N P L, n)}(t)=h_{l j}^{(N P, n)}(t)+a_{j}^{L}(t)=\widehat{h}_{l j}^{(N P, n)}+\breve{h}_{l j}^{(N P, n)}(t)+\breve{a}_{j}^{L}+t \cdot\left(\breve{a}_{j}-\breve{a}_{j}^{L}\right) \text { for } j \in N \\
& \bar{h}_{l j}^{(P N U, n)}(t)=h_{l j}^{(P N, n)}(t)+a_{j}^{U}(t)=\widehat{h}_{l j}^{(P N, n)}+\breve{h}_{l j}^{(P N, n)}(t)+\breve{a}_{j}^{U}+t \cdot\left(\breve{a}_{j}-\breve{a}_{j}^{U}\right) \text { for } j \in N .
\end{aligned}
$$

given by

From (257) and (258), we see that $\bar{h}_{l j}^{(P N L, n)}, \bar{h}_{l j}^{(N P U, n)}, \bar{h}_{l j}^{(N P L, n)}$ and $\bar{h}_{l j}^{(P N U, n)}$ are linear functions of $t$

$$
\begin{gathered}
\bar{h}_{l j}^{(P N L, n)}(t)=h_{l j}^{(P N, n)}(t)+a_{j}^{L}(t)=\mathfrak{r}_{l j}^{(P N L, n)} \cdot t+\mathfrak{s}_{l j}^{(P N L, n)} \text { for } j \in P \\
\bar{h}_{l j}^{(N P U, n)}(t)=h_{l j}^{(N P, n)}(t)+a_{j}^{U}(t)=\mathfrak{r}_{l j}^{(N P U, n)} \cdot t+\mathfrak{s}_{l j}^{(N P U, n)} \text { for } j \in P \\
\bar{h}_{l j}^{(N P L, n)}(t)=h_{l j}^{(N P, n)}(t)+a_{j}^{L}(t)=\mathfrak{r}_{l j}^{(N P L, n)} \cdot t+\mathfrak{s}_{l j}^{(N P L, n)} \text { for } j \in N \\
\bar{h}_{l j}^{(P N U, n)}(t)=h_{l j}^{(P N, n)}(t)+a_{j}^{U}(t)=\mathfrak{r}_{l j}^{(P N U, n)} \cdot t+\mathfrak{s}_{l j}^{(P N U, n)} \text { for } j \in N,
\end{gathered}
$$

where

$$
\mathfrak{r}_{l j}^{(P N L, n)}=\sum_{\left\{i: j \in P_{i}\right\}}\left(\breve{b}_{i j}^{L}-\breve{b}_{i j}\right) \cdot w_{l i}^{(* I, n)}+\sum_{\left\{i: j \in N_{i}\right\}}\left(\breve{b}_{i j}^{U}-\breve{b}_{i j}\right) \cdot w_{l i}^{(* I I, n)}+\breve{a}_{j}-\breve{a}_{j}^{L} \text { for } j \in P
$$




$$
\begin{aligned}
& \mathfrak{r}_{l j}^{(N P U, n)}=\sum_{\left\{i: j \in N_{i}\right\}}\left(\breve{b}_{i j}^{L}-\breve{b}_{i j}\right) \cdot w_{l i}^{(* I, n)}+\sum_{\left\{i: j \in P_{i}\right\}}\left(\breve{b}_{i j}^{U}-\breve{b}_{i j}\right) \cdot w_{l i}^{(* I, n)}+\breve{a}_{j}-\breve{a}_{j}^{U} \text { for } j \in P \\
& \mathfrak{r}_{l j}^{(N P L, n)}=\sum_{\left\{i: j \in N_{i}\right\}}\left(\breve{b}_{i j}^{L}-\breve{b}_{i j}\right) \cdot w_{l i}^{(* I, n)}+\sum_{\left\{i: j \in P_{i}\right\}}\left(\breve{b}_{i j}^{U}-\breve{b}_{i j}\right) \cdot w_{l i}^{(* I I, n)}+\breve{a}_{j}-\breve{a}_{j}^{L} \text { for } j \in N \\
& \mathfrak{r}_{l j}^{(P N U, n)}=\sum_{\left\{i: j \in P_{i}\right\}}\left(\breve{b}_{i j}^{L}-\breve{b}_{i j}\right) \cdot w_{l i}^{(* I, n)}+\sum_{\left\{i: j \in N_{i}\right\}}\left(\breve{b}_{i j}^{U}-\breve{b}_{i j}\right) \cdot w_{l i}^{(* I I, n)}+\breve{a}_{j}-\breve{a}_{j}^{U} \text { for } j \in N
\end{aligned}
$$

and

$$
\begin{gathered}
\mathfrak{s}_{l j}^{(P N L, n)}=\widehat{h}_{l j}^{(P N, n)}-\sum_{\left\{i: j \in P_{i}\right\}} \breve{b}_{i j}^{L} \cdot w_{l i}^{(* I, n)}-\sum_{\left\{i: j \in N_{i}\right\}} \breve{b}_{i j}^{U} \cdot w_{l i}^{(* I I, n)}+\breve{a}_{j}^{L} \\
=\frac{l}{n} \cdot \sum_{\left\{i: j \in P_{i}\right\}}\left(\breve{b}_{i j}-\breve{b}_{i j}^{L}\right) \cdot w_{l i}^{(* I, n)}+\frac{l-1}{n} \cdot \sum_{\left\{i: j \in N_{i}\right\}}\left(\breve{b}_{i j}-\breve{b}_{i j}^{U}\right) \cdot w_{l i}^{(* I I, n)}+\breve{a}_{j}^{L} \text { for } j \in P \\
\mathfrak{s}_{l j}^{(N P U, n)}=\widehat{h}_{l j}^{(N P, n)}-\sum_{\left\{i: j \in N_{i}\right\}} \breve{b}_{i j}^{L} \cdot w_{l i}^{(* I, n)}-\sum_{\left\{i: j \in P_{i}\right\}} \breve{b}_{i j}^{U} \cdot w_{l i}^{(* I I, n)}+\breve{a}_{j}^{U} \\
=\frac{l}{n} \cdot \sum_{\left\{i: j \in N_{i}\right\}}\left(\breve{b}_{i j}-\breve{b}_{i j}^{L}\right) \cdot w_{l i}^{(* I, n)}+\frac{l-1}{n} \cdot \sum_{\left\{i: j \in P_{i}\right\}}\left(\breve{b}_{i j}-\breve{b}_{i j}^{U}\right) \cdot w_{l i}^{(* I I, n)}+\breve{a}_{j}^{U} \text { for } j \in P \\
\mathfrak{s}_{l j}^{(N P L, n)}=\frac{l}{n} \cdot \sum_{\left\{i: j \in N_{i}\right\}}\left(\breve{b}_{i j}-\breve{b}_{i j}^{L}\right) \cdot w_{l i}^{(* I, n)}+\frac{l-1}{n} \cdot \sum_{\left\{i: j \in P_{i}\right\}}\left(\breve{b}_{i j}-\breve{b}_{i j}^{U}\right) \cdot w_{l i}^{(* I I, n)}+\breve{a}_{j}^{L} \text { for } j \in N \\
\mathfrak{s}_{l j}^{(P N U, n)}=\frac{l}{n} \cdot \sum_{\left\{i: j \in P_{i}\right\}}\left(\breve{b}_{i j}-\breve{b}_{i j}^{L}\right) \cdot w_{l i}^{(* I, n)}+\frac{l-1}{n} \cdot \sum_{\left\{i: j \in N_{i}\right\}}\left(\breve{b}_{i j}-\breve{b}_{i j}^{U}\right) \cdot w_{l i}^{(* I, n)}+\breve{a}_{j}^{U} \text { for } j \in N .
\end{gathered}
$$

Therefore, from (72)-(75), we obtain

$$
\begin{aligned}
\pi_{l}^{(P N L, n)} & =\max _{j \in P}\left\{-a_{l j}^{(L, n)}+\max _{t \in \bar{E}_{l}^{(n)}} \bar{h}_{l j}^{(P N L, n)}(t)\right\} \\
& =\max _{j \in P}\left\{-\frac{1}{n} \cdot\left[(n-l+1) \cdot \breve{a}_{j}^{L}+(l-1) \cdot \breve{a}_{j}\right]+\max _{t \in \bar{E}_{l}^{(n)}} \bar{h}_{l j}^{(P N L, n)}(t)\right\} \\
\pi_{l}^{(N P U, n)} & =\max _{j \in P}\left\{-a_{l j}^{(U, n)}+\max _{t \in \bar{E}_{l}^{(n)}} \bar{h}_{l j}^{(N P U, n)}(t)\right\} \\
& =\max _{j \in P}\left\{-\frac{1}{n} \cdot\left[(n-l) \cdot \breve{a}_{j}^{U}+l \cdot \breve{a}_{j}\right]+\max _{t \in \bar{E}_{l}^{(n)}} \bar{h}_{l j}^{(N P U, n)}(t)\right\}, \\
\pi_{l}^{(N P L, n)} & =\max _{j \in N}\left\{-a_{l j}^{(L, n)}+\max _{t \in \bar{E}_{l}^{(n)}} \bar{h}_{l j}^{(N P L, n)}(t)\right\} \\
& =\max _{j \in N}\left\{-\frac{1}{n} \cdot\left[(n-l+1) \cdot \breve{a}_{j}^{L}+(l-1) \cdot \breve{a}_{j}\right]+\max _{t \in \bar{E}_{l}^{(n)}} \bar{h}_{l j}^{(N P L, n)}(t)\right\}
\end{aligned}
$$




$$
\begin{aligned}
\pi_{l}^{(P N U, n)} & =\max _{j \in N}\left\{-a_{l j}^{(U, n)}+\max _{t \in \bar{E}_{l}^{(n)}} \bar{h}_{l j}^{(P N U, n)}(t)\right\} \\
& =\max _{j \in N}\left\{-\frac{1}{n} \cdot\left[(n-l) \cdot \breve{a}_{j}^{U}+l \cdot \breve{a}_{j}\right]+\max _{t \in \bar{E}_{l}^{(n)}} \bar{h}_{l j}^{(P N U, n)}(t)\right\},
\end{aligned}
$$

which can be calculated according to (225)-(228). Then $\pi_{l}^{(P N, n)}$ and $\pi_{l}^{(N P, n)}$ can be obtained from (76).

From (106) and (251), the error $\varepsilon_{n}$ between the approximate objective value and the optimal objective value is given by

$$
\begin{aligned}
\varepsilon_{n}= & -V\left(\mathrm{D}_{n}\right)+\sum_{l=1}^{n} \sum_{i=1}^{p}\left[\left(w_{l i}^{(* I, n)}+\max \left\{\frac{\pi_{l}^{(P N, n)}}{\mathfrak{b}_{l}^{(P N, n)},} \frac{\pi_{l}^{(N P, n)}}{\mathfrak{b}_{l}^{(N P, n)}}\right\}\right) \cdot \int_{\frac{l-1}{n}}^{\frac{l}{n}}\left((1-t) \cdot \breve{c}_{i}^{L}+t \cdot \breve{c}_{i}\right) d t\right] \\
& +\sum_{l=1}^{n} \sum_{i=1}^{p}\left[\left(w_{l i}^{(* I I, n)}+\max \left\{\frac{\pi_{l}^{(P N, n)}}{\mathfrak{b}_{l}^{(P N, n)},}, \frac{\pi_{l}^{(N P, n)}}{\mathfrak{b}_{l}^{(N P, n)}}\right\}\right) \cdot \int_{\frac{l-1}{n}}^{\frac{l}{n}}\left((1-t) \cdot \breve{c}_{i}^{U}+t \cdot \breve{c}_{i}\right) d t\right] \\
= & -V\left(\mathrm{D}_{n}\right)+\sum_{l=1}^{n} \sum_{i=1}^{p}\left[\left(w_{l i}^{(* I, n)}+\max \left\{\frac{\pi_{l}^{(P N, n)}}{\mathfrak{b}_{l}^{(P N, n)},} \frac{\pi_{l}^{(N P, n)}}{\mathfrak{b}_{l}^{(N P, n)}}\right\}\right) \cdot\left(\frac{\breve{c}_{i}^{L}}{n}+\left(\breve{c}_{i}-\breve{c}_{i}^{L}\right) \cdot \frac{2 l-1}{2 n^{2}}\right)\right] \\
& +\sum_{l=1}^{n} \sum_{i=1}^{p}\left[\left(w_{l i}^{(* I I, n)}+\max \left\{\frac{\pi_{l}^{(P N, n)}}{\mathfrak{b}_{l}^{(P N, n)}}, \frac{\pi_{l}^{(N P, n)}}{\mathfrak{b}_{l}^{(N P, n)}}\right\}\right) \cdot\left(\frac{\breve{c}_{i}^{U}}{n}+\left(\breve{c}_{i}-\breve{c}_{i}^{U}\right) \cdot \frac{2 l-1}{2 n^{2}}\right)\right] .
\end{aligned}
$$

Now suppose that we consider the nonnegative constraints. By referring to (208), the error $\varepsilon_{n}$ is given by

$$
\begin{aligned}
\varepsilon_{n}= & -V\left(\mathrm{D}_{n}\right)+\sum_{l=1}^{n} \sum_{i=1}^{p}\left[\left(w_{l i}^{(* I, n)}+\max \left\{\frac{\pi_{l}^{(L, n)}}{\mathfrak{b}_{l}^{(L, n)}}, \frac{\pi_{l}^{(U, n)}}{\mathfrak{b}_{l}^{(U, n)}}\right\}\right) \cdot\left(\frac{\breve{c}_{i}^{L}}{n}+\left(\breve{c}_{i}-\breve{c}_{i}^{L}\right) \cdot \frac{2 l-1}{2 n^{2}}\right)\right] \\
& +\sum_{l=1}^{n} \sum_{i=1}^{p}\left[\left(w_{l i}^{(* I, n)}+\max \left\{\frac{\pi_{l}^{(L, n)}}{\mathfrak{b}_{l}^{(L, n)}}, \frac{\pi_{l}^{(U, n)}}{\mathfrak{b}_{l}^{(U, n)}}\right\}\right) \cdot\left(\frac{\breve{c}_{i}^{U}}{n}+\left(\breve{c}_{i}-\breve{c}_{i}^{U}\right) \cdot \frac{2 l-1}{2 n^{2}}\right)\right] .
\end{aligned}
$$

However, from (202), the tighter error bound $\varepsilon_{n}^{\circ}$ is given by

$$
\begin{aligned}
\varepsilon_{n}^{\circ}= & -V\left(\mathrm{D}_{n}\right)+\sum_{l=1}^{n} \sum_{i=1}^{p}\left[\left(w_{l i}^{(* I, n)}+\frac{\pi_{l}^{(L, n)}}{\mathfrak{b}_{l}^{(L, n)}}\right) \cdot\left(\frac{\breve{c}_{i}^{L}}{n}+\left(\breve{c}_{i}-\breve{c}_{i}^{L}\right) \cdot \frac{2 l-1}{2 n^{2}}\right)\right] \\
& +\sum_{l=1}^{n} \sum_{i=1}^{p}\left[\left(w_{l i}^{(* I I, n)}+\frac{\pi_{l}^{(U, n)}}{\mathfrak{b}_{l}^{(U, n)}}\right) \cdot\left(\frac{\breve{c}_{i}^{U}}{n}+\left(\breve{c}_{i}-\breve{c}_{i}^{U}\right) \cdot \frac{2 l-1}{2 n^{2}}\right)\right],
\end{aligned}
$$

where

$$
\mathfrak{b}_{l}^{(L, n)}=\frac{1}{n} \cdot \min _{j=1, \cdots, q}\left\{(n-l+1) \cdot \sum_{i=1}^{p} \breve{b}_{i j}^{L}+(l-1) \cdot \sum_{i=1}^{p} \breve{b}_{i j}\right\}
$$

and

$$
\mathfrak{b}_{l}^{(U, n)}=\frac{1}{n} \cdot \min _{j=1, \cdots, q}\left\{(n-l) \cdot \sum_{i=1}^{p} \breve{b}_{i j}^{U}+l \cdot \sum_{i=1}^{p} \breve{b}_{i j}\right\} .
$$

are obtained from (253) and (254), respectively, by taking $N_{i}=\varnothing$ for $i=1, \cdots, p$, and

$$
\pi_{l}^{(L, n)}=\max _{j=1, \cdots, q}\left\{-\frac{1}{n} \cdot\left[(n-l+1) \cdot \breve{a}_{j}^{L}+(l-1) \cdot \breve{a}_{j}\right]+\max _{t \in \bar{E}_{l}^{(n)}} \bar{h}_{l j}^{(L, n)}(t)\right\}
$$


and

$$
\pi_{l}^{(U, n)}=\max _{j=1, \cdots, q}\left\{-\frac{1}{n} \cdot\left[(n-l) \cdot \breve{a}_{j}^{U}+l \cdot \breve{a}_{j}\right]+\max _{t \in \bar{E}_{l}^{(n)}} \bar{h}_{l j}^{(U, n)}(t)\right\}
$$

are obtained from (267) and (268), respectively, by taking $N=N_{i}=\varnothing$ for $i=1, \cdots, p$. More precisely, we have

$$
\bar{h}_{l j}^{(L, n)}(t)=\mathfrak{r}_{l j}^{(L, n)} \cdot t+\mathfrak{s}_{l j}^{(L, n)} \text { and } \bar{h}_{l j}^{(U, n)}(t)=\mathfrak{r}_{l j}^{(U, n)} \cdot t+\mathfrak{s}_{l j}^{(U, n)}
$$

for $j=1, \cdots, q$, where $\mathfrak{r}_{l j}^{(L, n)}, \mathfrak{r}_{l j}^{(U, n)}, \mathfrak{s}_{l j}^{(L, n)}$ and $\mathfrak{s}_{l j}^{(U, n)}$ are obtained from (259), (260), (263) and (264), respectively, by taking $N_{i}=\varnothing$ for $i=1, \cdots, p$, which are given by

$$
\begin{gathered}
\mathfrak{r}_{l j}^{(L, n)}=\sum_{i=1}^{p}\left(\breve{b}_{i j}^{L}-\breve{b}_{i j}\right) \cdot w_{l i}^{(* I, n)}+\breve{a}_{j}-\breve{a}_{j}^{L} \\
\mathfrak{r}_{l j}^{(U, n)}=\sum_{i=1}^{p}\left(\breve{b}_{i j}^{U}-\breve{b}_{i j}\right) \cdot w_{l i}^{(* I I, n)}+\breve{a}_{j}-\breve{a}_{j}^{U} \\
\mathfrak{s}_{l j}^{(L, n)}=\frac{l}{n} \cdot \sum_{i=1}^{p}\left(\breve{b}_{i j}-\breve{b}_{i j}^{L}\right) \cdot w_{l i}^{(* I, n)}+\breve{a}_{j}^{L} \\
\mathfrak{s}_{l j}^{(U, n)}=\frac{l-1}{n} \cdot \sum_{i=1}^{p}\left(\breve{b}_{i j}-\breve{b}_{i j}^{U}\right) \cdot w_{l i}^{(* I I, n)}+\breve{a}_{j}^{U} .
\end{gathered}
$$

The maximum are obtained in the following forms

$$
\max _{t \in \bar{E}_{l}^{(n)}} \bar{h}_{l j}^{(L, n)}(t)= \begin{cases}\mathfrak{s}_{l j}^{(L, n)}, & \text { if } \mathfrak{r}_{l j}^{(L, n)}=0 \\ \mathfrak{r}_{l j}^{(L, n)} \cdot \frac{l}{n}+\mathfrak{s}_{l j}^{(L, n)}, & \text { if } \mathfrak{r}_{l j}^{(L, n)}>0 \\ \mathfrak{r}_{l j}^{(L, n)} \cdot \frac{l-1}{n}+\mathfrak{s}_{l j}^{(L, n)}, & \text { if } \mathfrak{r}_{l j}^{(L, n)}<0\end{cases}
$$

and

$$
\max _{t \in \bar{E}_{l}^{(n)}} \bar{h}_{l j}^{(U, n)}(t)= \begin{cases}\mathfrak{s}_{l j}^{(U, n),} & \text { if } \mathfrak{r}_{l j}^{(U, n)}=0 \\ \mathfrak{r}_{l j}^{(U, n)} \cdot \frac{l}{n}+\mathfrak{s}_{l j}^{(U, n),} & \text { if } \mathfrak{r}_{l j}^{(U, n)}>0 \\ \mathfrak{r}_{l j}^{(U, n)} \cdot \frac{l-1}{n}+\mathfrak{s}_{l j}^{(U, n)}, & \text { if } \mathfrak{r}_{l j}^{(U, n)}<0 .\end{cases}
$$

Next we provide a numerical example that assumes each fuzzy number to be nonnegative.

Example 2. We consider a problem of allocating three resources to the annual production of three commodities by a manufacturing firm. The amounts of the three products to be produced are denoted by $x_{1}, x_{2}, x_{3}$. The objective function reflects the dollars contribution to profit of these products. We also assume that the data cannot be known or measured precisely. Therefore the problem is formulated as a fuzzy linear programming problem with fuzzy decision variables as shown below:

$$
\begin{array}{ll}
\max & \left(\widetilde{10} \otimes \widetilde{x}_{1}\right) \oplus\left(\widetilde{15} \otimes \widetilde{x}_{2}\right) \oplus\left(\widetilde{5} \otimes \widetilde{x}_{3}\right) \\
\text { subject to } \quad & \left(\widetilde{2} \otimes \widetilde{x}_{1}\right) \oplus\left(\widetilde{1} \otimes \widetilde{x}_{2}\right) \preceq \widetilde{6000} \\
& \left(\widetilde{3} \otimes \widetilde{x}_{1}\right) \oplus\left(\widetilde{3} \otimes \widetilde{x}_{2}\right) \oplus\left(\widetilde{1} \otimes \widetilde{x}_{3}\right) \preceq \widetilde{9000} \\
& \left.\left(\widetilde{1} \otimes \widetilde{x}_{1}\right) \oplus\left(\widetilde{2} \otimes \widetilde{x}_{2}\right) \oplus \widetilde{2} \otimes \widetilde{x}_{3}\right) \preceq \widetilde{4000} \\
& \widetilde{x}_{1}, \widetilde{x}_{2}, \widetilde{x}_{3} \text { are nonnegative fuzzy numbers, }
\end{array}
$$


where the triangular fuzzy numbers are taken as follows:

$$
\begin{array}{lll}
\widetilde{1}=(0.75,1,1.25), & \widetilde{2}=(1.5,2,2.5), & \widetilde{3}=(2.5,3,3.5) \\
\widetilde{5}=(4,5,6), & \widetilde{10}=(8,10,12), & \widetilde{15}=(13,15,17) \\
\widetilde{4000}=(3900,4000,4100), & \widetilde{6000}=(5850,6000,6150), & \widetilde{9000}=(8800,9000,9200) .
\end{array}
$$

Therefore we have

$$
\begin{aligned}
& \widetilde{a}_{1}=\left(\breve{a}_{1}^{L}, \breve{a}_{1}, \breve{a}_{1}^{U}\right)=\widetilde{10}=(8,10,12) \\
& \widetilde{a}_{2}=\left(\breve{a}_{2}^{L}, \breve{a}_{2}, \breve{a}_{2}^{U}\right)=\widetilde{15}=(13,15,17) \\
& \widetilde{a}_{3}=\left(\breve{a}_{3}^{L}, \breve{a}_{3}, \breve{a}_{3}^{U}\right)=\widetilde{5}=(4,5,6) \\
& \widetilde{c}_{1}=\left(\breve{c}_{1}^{L}, \breve{c}_{1}, \breve{c}_{1}^{U}\right)=\widetilde{6000}=(5850,6000,6150) \\
& \widetilde{c}_{2}=\left(\breve{c}_{2}^{L}, \breve{c}_{2}, \breve{c}_{2}^{U}\right)=\widetilde{9000}=(8800,9000,9200) \\
& \widetilde{c_{3}}=\left(\breve{c}_{3}^{L}, \breve{c}_{3}, \breve{c}_{3}^{U}\right)=\widetilde{4000}=(3900,4000,4100) \\
& {\left[\begin{array}{lll}
\widetilde{b}_{11} & \widetilde{b}_{12} & \widetilde{b}_{13} \\
\widetilde{b}_{21} & \widetilde{b}_{22} & \widetilde{b}_{23} \\
\widetilde{b}_{31} & \widetilde{b}_{32} & \widetilde{b}_{33}
\end{array}\right]=\left[\begin{array}{lll}
\left.\breve{b}_{11}^{L}, \breve{b}_{11}, \breve{b}_{11}^{U}\right) & \left(\breve{b}_{12}^{L}, \breve{b}_{12}, \breve{b}_{12}^{U}\right) & \left(\breve{b}_{13}^{L}, \breve{b}_{13}, \breve{b}_{13}^{U}\right) \\
\left(\breve{b}_{21}^{L}, \breve{b}_{21}, \breve{b}_{21}^{U}\right) & \left(\breve{b}_{22}^{L}, \breve{b}_{22}, \breve{b}_{22}^{U}\right) & \left(\breve{b}_{23}^{L}, \breve{b}_{23}, \breve{b}_{23}^{U}\right. \\
\left(\breve{b}_{31}^{L}, \breve{b}_{31}, \breve{b}_{31}^{U}\right) & \left(\breve{b}_{32}^{L}, \breve{b}_{32}, \breve{b}_{32}^{U}\right) & \left(\breve{b}_{33}^{L}, \breve{b}_{33}, \breve{b}_{33}^{U}\right)
\end{array}\right]} \\
& =\left[\begin{array}{ccc}
\widetilde{2} & \widetilde{1} & \widetilde{0} \\
\widetilde{3} & \widetilde{3} & \widetilde{1} \\
\widetilde{1} & \widetilde{2} & \widetilde{2}
\end{array}\right]=\left[\begin{array}{ccc}
(1.5,2,2.5) & (0.75,1,1.25) & (0,0,0) \\
(2.5,3,3.5) & (2.5,3,3.5) & (0.75,1,1.25) \\
(0.75,1,1.25) & (1.5,2,2.5) & (1.5,2,2.5)
\end{array}\right] .
\end{aligned}
$$

Since each fuzzy number is nonnegative, it follows that the index sets $N=N_{i}=\varnothing$ for $i=1,2,3$.

We are going to calculate the tighter error bound $\varepsilon_{n}^{\circ}$ using (272). From (273) and (274), we have

$$
\begin{aligned}
\mathfrak{b}_{l}^{(L, n)} & =\frac{1}{n} \cdot \min _{j=1, \cdots, q}\left\{(n-l+1) \cdot \sum_{i=1}^{p} \breve{b}_{i j}^{L}+(l-1) \cdot \sum_{i=1}^{p} \breve{b}_{i j}\right\} \\
& =\frac{1}{n} \cdot \min \{4.75(n-l+1)+6(l-1), 4.75(n-l+1)+6(l-1), 2.25(n-l+1)+3(l-1)\} \\
& =\frac{1}{n} \cdot[2.25(n-l+1)+3(l-1)]=2.25+0.75 \cdot \frac{l-1}{n}
\end{aligned}
$$

and

$$
\begin{aligned}
\mathfrak{b}_{l}^{(U, n)} & =\frac{1}{n} \cdot \min _{j=1, \cdots, q}\left\{(n-l) \cdot \sum_{i=1}^{p} \breve{b}_{i j}^{U}+l \cdot \sum_{i=1}^{p} \breve{b}_{i j}\right\} \\
& =\frac{1}{n} \cdot \min \{7.25(n-l)+6 l, 7.25(n-l)+6 l, 3.75(n-l)+3 l\} \\
& =\frac{1}{n} \cdot[3.75(n-l)+3 l]=3.75-0.75 \cdot \frac{l}{n} .
\end{aligned}
$$


From (277)-(280), we have

$$
\begin{aligned}
& \mathfrak{r}_{l 1}^{L}=-0.5 w_{l 1}^{(* I, n)}-0.5 w_{l 2}^{(* I, n)}-0.25 w_{l 3}^{(* I, n)}+2 \\
& \mathfrak{r}_{l 2}^{L}=-0.25 w_{l 1}^{(* I, n)}-0.5 w_{l 2}^{(* I, n)}-0.5 w_{l 3}^{(* I, n)}+2 \\
& \mathfrak{r}_{l 3}^{L}=-0.25 w_{l 2}^{(* I, n)}-0.5 w_{l 3}^{(* I, n)}+1
\end{aligned}
$$

and

$$
\begin{aligned}
\mathfrak{r}_{l 1}^{U} & =0.5 w_{l 1}^{(* I I, n)}+0.5 w_{l 2}^{(* I I, n)}+0.25 w_{l 3}^{(* I I, n)}-2 \\
\mathfrak{r}_{l 2}^{U} & =0.25 w_{l 1}^{(* I I, n)}+0.5 w_{l 2}^{(* I I, n)}+0.5 w_{l 3}^{(* I I, n)}-2 \\
\mathfrak{r}_{l 3}^{U} & =0.25 w_{l 2}^{(* I I, n)}+0.5 w_{l 3}^{(* I I, n)}-1
\end{aligned}
$$

and

$$
\begin{aligned}
\mathfrak{s}_{l 1}^{L} & =\frac{l}{n} \cdot\left(0.5 w_{l 1}^{(* I, n)}+0.5 w_{l 2}^{(* I, n)}+0.25 w_{l 3}^{(* I, n)}\right)+8 \\
\mathfrak{s}_{l 2}^{L} & =\frac{l}{n} \cdot\left(0.25 w_{l 1}^{(* I, n)}+0.5 w_{l 2}^{(* I, n)}+0.5 w_{l 3}^{(* I, n)}\right)+13 \\
\mathfrak{s}_{l 3}^{L} & =\frac{l}{n} \cdot\left(0.25 w_{l 2}^{(* I, n)}+0.5 w_{l 3}^{(* I, n)}\right)+4
\end{aligned}
$$

and

$$
\begin{aligned}
\mathfrak{s}_{l 1}^{U} & =\frac{1-l}{n} \cdot\left(0.5 w_{l 1}^{(* I I, n)}+0.5 w_{l 2}^{(* I I, n)}+0.25 w_{l 3}^{(* I I, n)}\right)+12 \\
\mathfrak{s}_{l 2}^{U} & =\frac{1-l}{n} \cdot\left(0.25 w_{l 1}^{(* I I, n)}+0.5 w_{l 2}^{(* I I, n)}+0.5 w_{l 3}^{(* I I, n)}\right)+17 \\
\mathfrak{s}_{l 3}^{U} & =\frac{1-l}{n} \cdot\left(0.25 w_{l 2}^{(* I I, n)}+0.5 w_{l 3}^{(* I I, n)}\right)+6 .
\end{aligned}
$$

According to (275) and (276), we have

$$
\begin{aligned}
\pi_{l}^{(L, n)}=\max & \left\{-\left(8+2 \cdot \frac{l-1}{n}\right)+\max _{t \in \bar{E}_{l}^{(n)}} \bar{h}_{l 1}^{(L, n)}(t),-\left(13+2 \cdot \frac{l-1}{n}\right)+\max _{t \in \bar{E}_{l}^{(n)}} \bar{h}_{l 2}^{(L, n)}(t),\right. \\
& \left.-\left(4+\frac{l-1}{n}\right)+\max _{t \in \bar{E}_{l}^{(n)}} \bar{h}_{l 3}^{(L, n)}(t)\right\}
\end{aligned}
$$

and

$$
\begin{aligned}
\pi_{l}^{(U, n)}=\max \{ & -\left(12-2 \cdot \frac{l}{n}\right)+\max _{t \in \bar{E}_{l}^{(n)}} \bar{h}_{l 1}^{(U, n)}(t),-\left(17-2 \cdot \frac{l}{n}\right)+\max _{t \in \bar{E}_{l}^{(n)}} \bar{h}_{l 2}^{(U, n)}(t), \\
& \left.-\left(6-\frac{l}{n}\right)+\max _{t \in \bar{E}_{l}^{(n)}} \bar{h}_{l 3}^{(U, n)}(t)\right\},
\end{aligned}
$$


which can be calculated according to (281) and (282). According to (272) and (271), the error bound $\varepsilon_{n}$ and tighter error bound $\varepsilon_{n}^{\circ}$ are given by

$$
\begin{aligned}
& \varepsilon_{n}=-V\left(D_{n}\right)+\sum_{l=1}^{n}\left[\left(w_{l 1}^{(* I, n)}+\max \left\{\frac{\pi_{l}^{(L, n)}}{\mathfrak{b}_{l}^{(L, n)}}, \frac{\pi_{l}^{(U, n)}}{\mathfrak{b}_{l}^{(U, n)}}\right\}\right) \cdot\left(\frac{5850}{n}+150 \cdot \frac{2 l-1}{2 n^{2}}\right)\right. \\
& +\left(w_{l 2}^{(* I, n)}+\max \left\{\frac{\pi_{l}^{(L, n)}}{\mathfrak{b}_{l}^{(L, n)}}, \frac{\pi_{l}^{(U, n)}}{\mathfrak{b}_{l}^{(U, n)}}\right\}\right) \cdot\left(\frac{8800}{n}+200 \cdot \frac{2 l-1}{2 n^{2}}\right) \\
& \left.+\left(w_{l 3}^{(* I, n)}+\max \left\{\frac{\pi_{l}^{(L, n)}}{\mathfrak{b}_{l}^{(L, n)}}, \frac{\pi_{l}^{(U, n)}}{\mathfrak{b}_{l}^{(U, n)}}\right\}\right) \cdot\left(\frac{3900}{n}+100 \cdot \frac{2 l-1}{2 n^{2}}\right)\right] \\
& +\sum_{l=1}^{n}\left[\left(w_{l 1}^{(* I I, n)}+\max \left\{\frac{\pi_{l}^{(L, n)}}{\mathfrak{b}_{l}^{(L, n)}}, \max \left\{\frac{\pi_{l}^{(L, n)}}{\mathfrak{b}_{l}^{(L, n)}}, \frac{\pi_{l}^{(U, n)}}{\mathfrak{b}_{l}^{(U, n)}}\right\}\right\}\right) \cdot\left(\frac{6150}{n}-150 \cdot \frac{2 l-1}{2 n^{2}}\right)\right. \\
& +\left(w_{l 2}^{(* I I, n)}+\frac{\pi_{l}^{(U, n)}}{\mathfrak{b}_{l}^{(U, n)}}\right) \cdot\left(\frac{9200}{n}-200 \cdot \frac{2 l-1}{2 n^{2}}\right) \\
& \left.+\left(w_{l 3}^{(* I I, n)}+\max \left\{\frac{\pi_{l}^{(L, n)}}{\mathfrak{b}_{l}^{(L, n)}}, \frac{\pi_{l}^{(U, n)}}{\mathfrak{b}_{l}^{(U, n)}}\right\}\right) \cdot\left(\frac{4100}{n}-100 \cdot \frac{2 l-1}{2 n^{2}}\right)\right] .
\end{aligned}
$$

and

$$
\begin{aligned}
& \varepsilon_{n}^{\circ}=-V\left(D_{n}\right)+\sum_{l=1}^{n} {\left[\left(w_{l 1}^{(* I, n)}+\frac{\pi_{l}^{(L, n)}}{\mathfrak{b}_{l}^{(L, n)}}\right) \cdot\left(\frac{5850}{n}+150 \cdot \frac{2 l-1}{2 n^{2}}\right)\right.} \\
&+\left(w_{l 2}^{(* I, n)}+\frac{\pi_{l}^{(L, n)}}{\mathfrak{b}_{l}^{(L, n)}}\right) \cdot\left(\frac{8800}{n}+200 \cdot \frac{2 l-1}{2 n^{2}}\right) \\
&\left.+\left(w_{l 3}^{(* I, n)}+\frac{\pi_{l}^{(L, n)}}{\mathfrak{b}_{l}^{(L, n)}}\right) \cdot\left(\frac{3900}{n}+100 \cdot \frac{2 l-1}{2 n^{2}}\right)\right] \\
&+\sum_{l=1}^{n}\left[\left(w_{l 1}^{(* I, n)}+\frac{\pi_{l}^{(U, n)}}{\mathfrak{b}_{l}^{(U, n)}}\right) \cdot\left(\frac{6150}{n}-150 \cdot \frac{2 l-1}{2 n^{2}}\right)\right. \\
&+\left(w_{l 2}^{(* I, n)}+\frac{\pi_{l}^{(U, n)}}{\mathfrak{b}_{l}^{(U, n)}}\right) \cdot\left(\frac{9200}{n}-200 \cdot \frac{2 l-1}{2 n^{2}}\right) \\
&\left.+\left(w_{l 3}^{(* I I, n)}+\frac{\pi_{l}^{(U, n)}}{\mathfrak{b}_{l}^{(U, n)}}\right) \cdot\left(\frac{4100}{n}-100 \cdot \frac{2 l-1}{2 n^{2}}\right)\right] .
\end{aligned}
$$

It is clear to see that $\varepsilon_{n}^{\circ} \leq \varepsilon_{n}$.

In this example, although we consider the fuzzy decision variables $\tilde{x}_{j}$ for $j=1,2,3$, we occasionally obtain the crisp optimal solution, which means

$$
x_{j}^{(L, n)}(t)=x_{j}^{(U, n)}(t) \equiv x_{j}^{(n)} \text { for all } t \in[0,1]
$$

are constant functions with the corresponding constants $x_{j}^{(n)}$ for $j=1,2,3$. 
The approximate optimal objective value of problem SOP is give by

$$
\begin{aligned}
V\left(S_{n}\right)=\sum_{j=1}^{q} \int_{0}^{1} a_{j}^{L}(t) \cdot x_{j}^{(L, n)}(t) d t+\sum_{j=1}^{q} \int_{0}^{1} a_{j}^{U}(t) \cdot x_{j}^{(U, n)}(t) d t \\
=\sum_{j=1}^{q} \sum_{l=1}^{n} z_{l j}^{(L, n)} \cdot \int_{\frac{l-1}{n}}^{\frac{l}{n}}\left[\breve{a}_{j}^{L}+t \cdot\left(\breve{a}_{j}-\breve{a}_{j}^{L}\right)\right] d t+\sum_{j=1}^{q} \sum_{l=1}^{n} z_{l j}^{(U, n)} \cdot \int_{\frac{l-1}{n}}^{\frac{l}{n}}\left[\breve{a}_{j}^{U}+t \cdot\left(\breve{a}_{j}-\breve{a}_{j}^{U}\right)\right] d t \\
\quad(u \operatorname{sing}(250)) \\
=\sum_{j=1}^{q} \sum_{l=1}^{n} z_{l j}^{(L, n)} \cdot\left[\frac{\breve{a}_{j}^{L}}{n}+\frac{\breve{a}_{j}-\breve{a}_{j}^{L}}{2 n^{2}} \cdot(2 l-1)\right] d t+\sum_{j=1}^{q} \sum_{l=1}^{n} z_{l j}^{(U, n)} \cdot \int_{\frac{l-1}{n}}^{\frac{l}{n}}\left[\frac{\breve{a}_{j}^{U}}{n}+\frac{\breve{a}_{j}-\breve{a}_{j}^{U}}{2 n^{2}} \cdot(2 l-1)\right] d t .
\end{aligned}
$$

Theorems 3 and 4 say that

$$
0 \leq V(S O P)-V\left(S O P_{n}\right) \leq \varepsilon_{n}
$$

and

$$
0 \leq V\left(S O P_{n}\right)-V\left(P_{n}\right) \leq V(S O P)-V\left(P_{n}\right) \leq \varepsilon_{n}
$$

In the following Table 2, we present the error estimation $\varepsilon_{n}^{\circ}$ for the different values of $n$ and the approximate crisp solution $x_{j}^{(n)}$. We also calculate the relative error of $\varepsilon_{n}^{\circ}$ with respect to $V\left(P_{n}\right)$ given by $\varepsilon_{n}^{\circ *}=\varepsilon_{n}^{\circ} / V\left(P_{n}\right)$. Note that $x_{3}^{(n)}=0$ is not presented in the following Table 2.

Table 2. Numerical results.

\begin{tabular}{ccccccc}
\hline $\boldsymbol{n}$ & $\varepsilon_{\boldsymbol{n}}^{\circ}$ & $\boldsymbol{V}\left(\mathrm{P}_{\boldsymbol{n}}\right)$ & $\boldsymbol{V}\left(\right.$ SOP $\left._{\boldsymbol{n}}\right)$ & $\boldsymbol{\varepsilon}_{\boldsymbol{n}}^{\circ *}$ & $\boldsymbol{x}_{\mathbf{1}}^{(\boldsymbol{n})}$ & $\boldsymbol{x}_{\mathbf{2}}^{(\boldsymbol{n})}$ \\
\hline 10 & 14819 & 60,962 & 61,435 & 0.2430910 & 2031.746 & 670.635 \\
100 & 1473.3 & 62,078 & 62,126 & 0.0237328 & 2060.317 & 674.206 \\
500 & 294.52 & 62,177 & 62,187 & 0.0047369 & 2062.857 & 674.524 \\
1000 & 147.25 & 62,190 & 62,195 & 0.0023678 & 2063.175 & 674.563 \\
2000 & 73.626 & 62,196 & 62,199 & 0.0011838 & 2063.333 & 674.583 \\
3000 & 49.083 & 62,198 & 62,200 & 0.0007891 & 2063.386 & 674.590 \\
\hline
\end{tabular}

Suppose that the decision-maker can tolerate the relative error $\varepsilon_{n}^{\circ *}=0.005$. Then we see that $n=500$ is sufficient to achieve this relative error $\varepsilon_{n}^{\circ *}$.

Suppose that each quantity in the original fuzzy linear programming problem is assumed to be a real number; that is, we consider the following linear programming problem

$$
\begin{array}{ll}
\max & 10 x_{1}+15 x_{2}+5 x_{3} \\
\text { subject to } & 2 x_{1}+x_{2} \leq 6000 \\
& 3 x_{1}+3 x_{2}+x_{3}+\leq 9000 \\
& x_{1}+2 x_{2}+2 x_{3} \leq 4000 \\
& x_{1}, x_{2}, x_{3} \in \mathbb{R}_{+} .
\end{array}
$$

Then the optimal solution is $\left(x_{1}, x_{2}, x_{3}\right)=(2000,1000,0)$ that is compatible with the solution presented above.

\section{Conclusions}

The fuzzy linear programming problems with fuzzy decision variables has been formulated in this paper based on the general type of bell-shaped membership functions rather than the triangular type of membership functions. In order to solve this difficult problem, it must be transformed into a scalar optimization problem in which the solution concepts and some related properties have been studied. We have also introduced a discretized problem of the transformed scalar optimization problem by equally dividing the unit interval $[0,1]$ into subintervals with equal lengths. In order 
to design the computational procedure, the dual problem of this discretized problem has also been formulated. The important issue is to derive an analytic formula of the error bound, which is presented in Theorem 3. On the other hand, the existence of optimal solutions has also been studied.

In this paper, we use MATLAB on PC to obtain the numerical results. Since the discretized problem is a large scale linear programming problem, it means that the "out of memory" error will frequently occur when the computational procedure is implemented on PC. There are many ways to overcome this situation.

- The simple way is to implement the computational procedure in super computer. Then we can satisfy error estimation.

- Since the large scale linear programming problem contains the sparse matrix, it is possible to design a computational procedure that can just handle the nonzero data. This can save the memory without storing the zero data in the sparse matrix. The numerical optimization involving the sparse matrix has already been studied. The readers may use the existing technique for handling the sparse matrix to improve the computational procedure proposed in this paper.

- The error estimation $\varepsilon_{n}$ and $\varepsilon_{n}^{\circ}$ derived in this paper may not be the better error estimation. This paper just provides a computational procedure which shows the research direction of deriving the error estimation. There may exist some other computational procedures that can improve the error estimation. In this case, we can achieve the satisfied relative error without running the large $n$ in the computer, which definitely can save the memory for avoiding out of memory. For example, for the problems with nonnegative constraints, from which we have derived tighter bounds of error estimation $\varepsilon_{n}^{\circ}$ that are smaller than the usual error estimation $\varepsilon_{n}$ for the general problems. Therefore, the readers who are interested in this topic may try to improve the error estimation; that is to say, to obtain a tighter bound of error estimation that can be presented as an analytic formula.

Problems in operations research can be studied under fuzzy environment when the data involves fuzziness. In the future research, we can solve the problems that are formulated with consideration of fuzzy decision variables by using the methodology proposed in this paper. As we described before, the existing articles always consider the triangular type of fuzzy decision variables. In this paper, the general bell-shaped type of fuzzy decision variables have been taken into account, which covers the special triangular type of fuzzy decision variables.

Funding: This research received no external funding

Conflicts of Interest: The author declares no conflict of interest.

\section{Appendix A}

We present the approximate optimal solutions in the following figures.

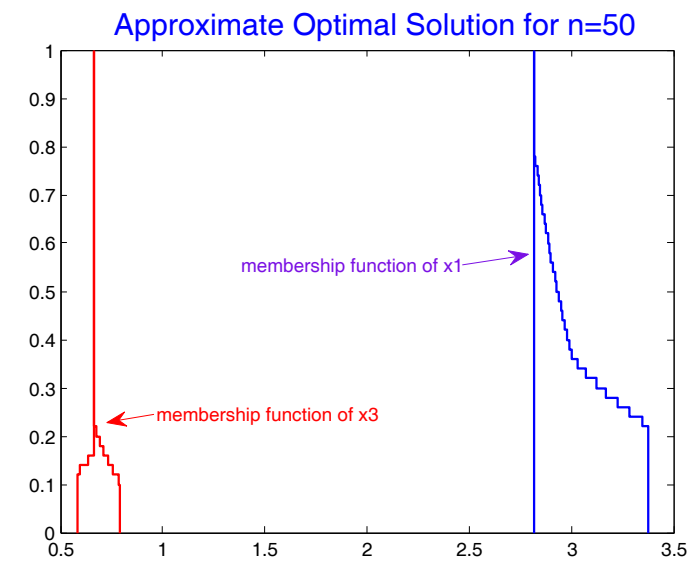

Figure A1. Approximate optimal solution for $n=50$. 


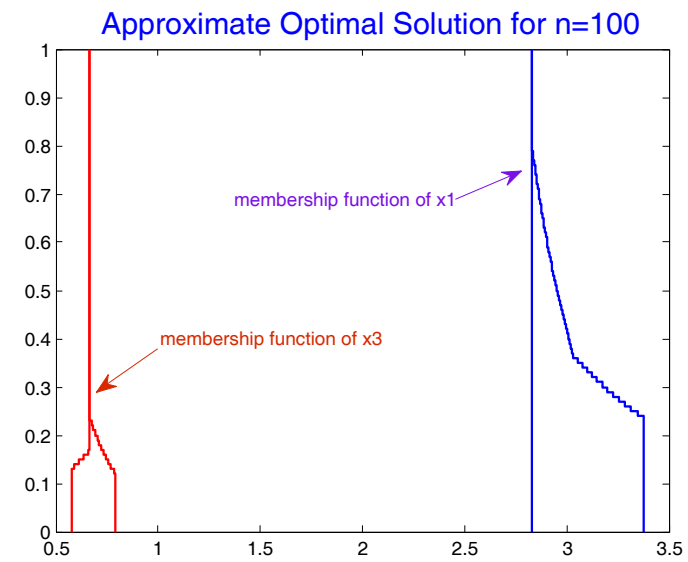

Figure A2. Approximate optimal solution for $n=100$.

\section{References}

1. Bellmam, R.E.; Zadeh, L.A. Decision-Making in a Fuzzy Environment. Manag. Sci. 1970, 17, $141-164$. [CrossRef]

2. Tanaka, H.; Okuda, T.; Asai, K. On Fuzzy-Mathematical Programming. J. Cybern. 1974, 3, 37-46. [CrossRef]

3. Zimmermann, H.-J. Description and Optimization of Fuzzy Systems. Int. J. Gen. Syst. 1976, 2, $209-215$. [CrossRef]

4. Zimmemmann, H.-J. Fuzzy Programming and Linear Programming with Several Objective Functions. Fuzzy Sets Syst. 1978, 1, 45-55. [CrossRef]

5. Herrera, F.; Kovács, M.; Verdegay, J.L. Optimality for fuzzified mathematical programming problems: A parametric approach. Fuzzy Sets Syst. 1993, 54, 279-285. [CrossRef]

6. Verdegay, J.L. A Dual Approach to Solve the Fuzzy Linear Programming Problems. Fuzzy Sets Syst. 1984, 14, 131-141. [CrossRef]

7. Buckley, J.J. Feuring, T. Evolutionary Algorithm Solution to Fuzzy Problems: Fuzzy Linear Programming. Fuzzy Sets Syst. 2000, 109, 35-53. [CrossRef]

8. Julien, B. An extension to possibilistic linear programming. Fuzzy Sets Syst. 1994, 64, 195-206. [CrossRef]

9. Luhandjula, M.K.; Ichihashi, H.; Inuiguchi, M. Fuzzy and semi-infinite mathematical programming. Inf. Sci. 1992, 61, 233-250. [CrossRef]

10. Inuiguchi, M. Necessity Measure Optimization in Linear Programming Problems with Fuzzy Polytopes. Fuzzy Sets Syst. 2007, 158, 1882-1891. [CrossRef]

11. Wu, H.-C. Duality Theory in Fuzzy Optimization Problems. Fuzzy Optim. Decis. Mak. 2004, 3, $345-365$. [CrossRef]

12. Wu, H.-C. Duality Theorems and Saddle Point Optimality Conditions in Fuzzy Nonlinear Programming Problems Based on Different Solution Concepts. Fuzzy Sets Syst. 2007, 158, 1588-1607. [CrossRef]

13. Wu, H.-C. The Optimality Conditions for Optimization Problems with Fuzzy-Valued Objective Functions. Optimization 2008, 57, 473-489. [CrossRef]

14. Wu, H.-C. The Karush-Kuhn-Tucker Optimality Conditions for Multi-objective Programming Problems with Fuzzy-Valued Objective Functions. Fuzzy Optim. Decis. Mak. 2009, 8, 1-28. [CrossRef]

15. Li, L.; Liu, S.; Zhang, J. On fuzzy generalized convex mappings and optimality conditions for fuzzy weakly univex mappings. Fuzzy Sets Syst. 2015, 280, 107-132. [CrossRef]

16. Chalco-Cano, Y.; Silva, G.N.; Rufian-Lizana, A. On the Newton method for solving fuzzy optimization problems. Fuzzy Sets Syst. 2015, 272, 60-69. [CrossRef]

17. Pirzada, U.M.; Pathak, V.D. Newton method for solving the multi-variable fuzzy optimization problem. J. Optim. Theorey Appl. 2013, 156, 867-881. [CrossRef]

18. Ezzati, R.; Khorram, E.; Enayati, R. A New Algorithm to Solve Fully Fuzzy Linear Programming Problems Using the MOLP Problem. Appl. Math. Model. 2015, 39, 3183-3193. [CrossRef] 
19. Ahmad, T.; Khan, M.; Khan, I.U.; Maan, N. Fully Fuzzy Linear Programming (FFLP) with a Special Ranking Function for Selection of Substitute Activities in Project Management. Int. J. Appl. Sci. Technol. 2011, 1, 234-246.

20. Jayalakshmi, M.; Pandian, P. A New Method for Finding an Optimal Fuzzy Solution for Fully Fuzzy Linear Programming Problems. Int. J. Eng. Res. Appl. 2012, 2, 247-254.

21. Khan, I.U.; Ahmad, T.; Maan, N. A Simplified Novel Technique for Solving Fully Fuzzy Linear Programming Problems. J. Optim. Theory Appl. 2013, 159, 536-546. [CrossRef]

22. Kumar, A.; Kaur, J.; Singh, P. A New Method for Solving Fully Fuzzy Linear Programming Problems. Appl. Math. Model. 2011, 35, 817-823. [CrossRef]

23. Lotfi, F.H.; Allahviranloo, T.; Jondabeh, M.A.; Alizadeh, L. Solving A Full Fuzzy Linear Programming Using Lexicography Method and Fuzzy Approximate Solution. Appl. Math. Model. 2009, 33, 3151-3156. [CrossRef]

24. Najafi, H.S.; Edalatpanah, S.A.; Dutta, H. A Nonlinear Model for Fully Fuzzy Linear Programming with Fully Unrestricted Variables and Parameters. Alex. Eng. J. 2016, 55, 2589-2595. [CrossRef]

25. Nasseri, S.H.; Behmanesh, E.; Taleshian, F.; Abdolalipoor, M.; Taghi-Nezhad, N.A. Fully Fuzzy Linear Programming with Inequality Constraints. Int. J. Ind. Math. 2013, 5, 309-316.

26. Kaur, A.; Kumar, A. Exact Fuzzy Optimal Solution of Fully Fuzzy Linear Programming Problems with Unrestricted Fuzzy Variables. Appl. Intell. 2012, 37, 145-154. [CrossRef]

27. Baykasoglu, A.; Gocken, T. A Direct Solution Approach to Fuzzy Mathematical Programs with Fuzzy Decision Variables. Expert Syst. Appl. 2012, 39, 1972-1978. [CrossRef]

28. Chakraborty, D.; Jana, D.K.; Roy, T.K. A New Approach to Solve Fully Fuzzy Transportation Problem Using Triangular Fuzzy Number. Int. J. Oper. Res. 2016, 26, 153-179. [CrossRef]

29. Jaikumar, K. New Approach to Solve Fully Fuzzy Transportation Problem. Int. J. Math. Its Appl. 2016, 4, 155-162.

30. Baykasoglu, A.; Subulan, K. Constrained Fuzzy Arithmetic Approach to Fuzzy Transportation Problems with Fuzzy Decision Variables. Expert Syst. Appl. 2017, 81, 193-222. [CrossRef]

31. Klir, G.J.; Pan, Y. Constrained Fuzzy Arithmetic: Basic Questions and Some Answers. Soft Comput. 1998, 2, 100-108. [CrossRef]

32. Ebrahimnejad, A. A Simplified New Approach for Solving Fuzzy Transportation Problems with Generalized Trapezoidal Fuzzy Numbers. Appl. Soft Comput. 2014, 19, 171-176. [CrossRef]

33. Kaur, A.; Kumar, A. A New Approach for Solving Fuzzy Transportation Problems Using Generalized Trapezoidal Fuzzy Numbers. Appl. Soft Comput. 2012, 12, 1201-1213. [CrossRef]

34. Royden, H.L. Real Analysis, 2nd ed.; Macmillan: London, UK, 1968.

35. Riesz, F.; Sz.-Nagy, B. Functional Analysis; Frederick Ungar Publishing Co.: New York, NY, USA, 1955.

36. Levinson, N. A class of continuous linear programming problems. J. Math. Anal. Appl. 1966, 16, 73-83. [CrossRef]

37. Dubois, D.; Prade, H. Operations on Fuzzy Numbers. Int. J. Syst. Sci. 1978, 9, 613-626. [CrossRef]

(C) 2019 by the authors. Licensee MDPI, Basel, Switzerland. This article is an open access article distributed under the terms and conditions of the Creative Commons Attribution (CC BY) license (http://creativecommons.org/licenses/by/4.0/). 